\title{
Phenomenology of Little Higgs Models at the Large Hadron Collider
}

by

\section{Kenneth Paul Moats}

A thesis submitted to the

Faculty of Graduate and Postdoctoral Affairs

in partial fulfillment of the requirements

for the degree of

Doctor of Philosophy

in

High Energy Physics

Department of Physics

Carleton University

Ottawa-Carleton Institute of Physics

Ottawa, Ontario, Canada

September 18, 2012

Copyright (c) 2012 Kenneth Paul Moats 
Library and Archives

Canada

Published Heritage

Branch

395 Wellington Street

Ottawa ON K1A ON4

Canada
Bibliothèque et

Archives Canada

Direction du

Patrimoine de l'édition

395 , rue Wellington

Ottawa ON K1A ON4

Canada
Your file Votre référence

ISBN: 978-0-494-93682-5

Our file Notre référence

ISBN: $978-0-494-93682-5$
NOTICE:

The author has granted a nonexclusive license allowing Library and Archives Canada to reproduce, publish, archive, preserve, conserve, communicate to the public by telecommunication or on the Internet, loan, distrbute and sell theses worldwide, for commercial or noncommercial purposes, in microform, paper, electronic and/or any other formats.

The author retains copyright ownership and moral rights in this thesis. Neither the thesis nor substantial extracts from it may be printed or otherwise reproduced without the author's permission.
AVIS:

L'auteur a accordé une licence non exclusive permettant à la Bibliothèque et Archives Canada de reproduire, publier, archiver, sauvegarder, conserver, transmettre au public par télécommunication ou par l'Internet, prêter, distribuer et vendre des thèses partout dans le monde, à des fins commerciales ou autres, sur support microforme, papier, électronique et/ou autres formats.

L'auteur conserve la propriété du droit d'auteur et des droits moraux qui protege cette thèse. $\mathrm{Ni}$ la thèse ni des extraits substantiels de celle-ci ne doivent être imprimés ou autrement reproduits sans son autorisation.
In compliance with the Canadian Privacy Act some supporting forms may have been removed from this thesis.

While these forms may be included in the document page count, their removal does not represent any loss of content from the thesis.
Conformément à la loi canadienne sur la protection de la vie privée, quelques formulaires secondaires ont été enlevés de cette thèse.

Bien que ces formulaires aient inclus dans la pagination, il n'y aura aucun contenu manquant. 


\section{Abstract}

Little Higgs models provide an elegant solution to the hierarchy problem of the Standard Model, introducing new particles at the $\mathrm{TeV}$ scale to cancel the quadratic divergences to the square of the Higgs boson mass. The research carried out in this thesis focuses on the Large Hadron Collider (LHC) phenomenology of two such Little Higgs models: the Littlest Higgs model and the Bestest Little Higgs model.

Firstly, the results of a study of Higgs triplet boson production in the Littlest Higgs model are presented in the $W^{ \pm} W^{ \pm}, W^{ \pm} Z, W^{+} W^{-}$, and $Z Z$ channels at the LHC for a centre of mass energy of $\sqrt{s}=14 \mathrm{TeV}$, comparing these results with the predictions of two additional Higgs triplet models: the Georgi-Machacek model and the Left-Right Symmetric model. It is found that, given the constraints on the triplet vacuum expectation value (vev), considerable luminosity is required to observe Higgs triplet bosons in vector boson scattering. Observing a Higgs triplet at the LHC is most promising in the Georgi-Machacek model due to a weaker constraint on the triplet vev. In this model, a Higgs triplet boson with a mass of $1.0(1.5) \mathrm{TeV}$ can be observed at the LHC with an integrated luminosity as low as $41(119) \mathrm{fb}^{-1}$ in the $W^{ \pm} W^{ \pm}$channel and as low as $171(474) \mathrm{fb}^{-1}$ in the $W^{ \pm} Z$ channel.

The structure of the Bestest Little Higgs model is then described, including the procedure for deriving the Feynman rules of this model. The results of a study of heavy quark production in the Bestest Little Higgs model at the LHC are presented,

focusing on associated single production of the exotic charge $5 / 3$ heavy quark, $T_{b}^{(5 / 3)}$, 
at $\sqrt{s}=14 \mathrm{TeV}$ for two scenarios of Yukawa couplings. Applying stringent kinematic cuts to reduce the backgrounds, it is found that, in the two scenarios considered, the $T_{b}^{(5 / 3)}$ heavy quark with a mass of 400,600 and $800 \mathrm{GeV}$ could be discovered in the same-sign dilepton channel at the LHC with an integrated luminosity as low as 43 , 149 and $797 \mathrm{fb}^{-1}$, respectively. 
To my brother, Brian William David Moats (1982-2006). 


\section{Acknowledgments}

I am most indebted to my co-advisors, Stephen Godfrey and Thomas Grégoire, for agreeing to take me on as their student. Their mentoring and patience are greatly appreciated, and their enthusiasm for research in particle physics has surely helped strengthen my interest in the subject.

I thank the members of the Carleton phenomenology group, particularly Pat Kalyniak and Travis Martin, as well as Heather Logan for helpful comments and discussions. I thank Hong Sheng Hou, Juergen Reuter and David Morrissey for helpful comments on my work on Higgs triplet production. I also thank Martin Schmaltz and Jesse Thaler for helpful discussions on the Bestest Little Higgs model, and Edmond Berger and Qing-Hong Cao for helpful discussions on heavy quark production.

I thank Bill Jack and Wade Hong for assistance in using the Carleton Department of Physics computing resources, without which many of the results in this thesis could not have been produced. I am most grateful to the Natural Sciences and Engineering Research Council of Canada (NSERC) and the Province of Ontario for funding via NSERC Post Graduate Scholarships and Ontario Graduate Scholarships.

Most importantly, I would like to thank my family for their unending love and support throughout my entire life. I am most appreciative of my fiancée, Sabrina Squire for her patience and encouragement while writing this thesis. I am especially grateful to my parents, Murray and Brenda Moats, for instilling in me the importance of education and for their endless supply of encouragement in all of my endeavors. 


\section{Statement of Originality}

Chapters 1 and 2 predominantly review material previously presented in the literature on the Standard Model of particle physics, and are referenced accordingly. Sections 3.1 and 3.2 review previous studies of vector boson scattering and Higgs triplet models, respectively, and are also referenced accordingly. The original research related to Higgs triplet production via vector boson scattering at the Large Hadron Collider is presented in Sections 3.3-3.6. This research has been published Physical Review D in collaboration with my advisor, Dr. Stephen Godfrey. All calculations presented here were originally performed by myself, and many of them were later verified independently by Dr. Godfrey. This research is entirely new and original and has not been submitted (to my knowledge) by anyone else for publication or as the final project of another degree.

Chapter 4 reviews previous research on the Bestest Little Higgs model, and is referenced accordingly. Chapter 5 presents original research related to heavy quark production in the Bestest Little Higgs model at the Large Hadron Collider. All calculations presented in Sections 5.2-5.4 were performed by myself and the calculations of Sections 5.2 and 5.3 were verified independently by Travis Martin. These results have been published in Journal of High Energy Physics in collaboration with Stephen Godfrey, Thomas Grégoire, Pat Kalyniak and Travis Martin. The calculations presented in Section 5.5 were performed entirely by myself. This research is entirely new and original and has not been submitted (to my knowledge) by anyone else for 
publication or as the final project of another degree.

Appendix A reviews some common notations and conventions used in particle physics calculations and is referenced accordingly. Appendix B contains additional details related to the Bestest Little Higgs model, including a derivation of the particle masses and Feynman rules in this model. These calculations, performed by myself, had yet to be carried out prior to this work and were verified independently by Thomas Grégoire, Pat Kalyniak and Travis Martin.

The material from this thesis should be cited as follows: Kenneth P. Moats, Phenomenology of Little Higgs Models at the Large Hadron Collider, Carleton University (Canada), 2012. 


\section{Table of Contents}

$\begin{array}{ll}\text { Abstract } & \text { ii }\end{array}$

$\begin{array}{ll}\text { Acknowledgments } & \text { v }\end{array}$

Statement of Originality $\quad$ vi

Table of Contents $\quad$ viii

List of Tables $\quad$ xi

List of Figures $\quad$ Xv

1 Introduction $\quad 1$

2 The Standard Model $\quad 5$

2.1 Introduction . . . . . . . . . . . . . . . 5

2.2 Quantum Chromodynamics (QCD) . . . . . . . . . . 8

2.3 The Electroweak Theory . . . . . . . . . . . . . . . 10

2.4 Electroweak Symmetry Breaking and the Higgs Mechanism . . . . . . 12

2.5 The Higgs Boson Mass . . . . . . . . . . . . . . . . . . 17

2.6 The Hierarchy Problem . . . . . . . . . . . . . . . 18

2.7 Little Higgs Models . . . . . . . . . . . . . . . . . . . . . . . . . . . 20 
3 Exploring Higgs Triplet Models Via Vector Boson Scattering at the $\begin{array}{ll}\text { Large Hadron Collider } & 27\end{array}$

3.1 Introduction . . . . . . . . . . . . . . . . . 27

3.2 Models . . . . . . . . . . . . . . . . . . . . . . . . . . . . . . . . . 29

3.2 .1 The Littlest Higgs Model . . . . . . . . . . . . . . . . 30

3.2 .2 The Georgi-Machacek Model . . . . . . . . . . . . . . 31

3.2 .3 The Left-Right Symmetric Model . . . . . . . . . . . . . 33

3.3 Calculations . . . . . . . . . . . . . . . . 34

3.4 Backgrounds . . . . . . . . . . . . . . . . . . . . . 38

3.4.1 The $W^{ \pm} W^{ \pm}$Channel . . . . . . . . . . . . . . . . . 42

3.4 .2 The $W^{ \pm} Z$ Channel . . . . . . . . . . . . . . . 44

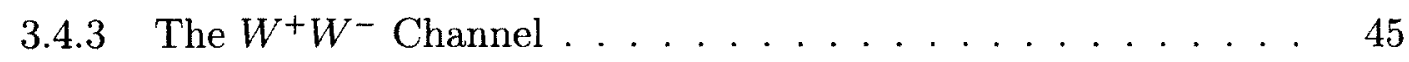

3.4.4 The $Z Z$ Channel . . . . . . . . . . . . . 46

3.4 .5 Kinematic Cuts . . . . . . . . . . . . 46

3.5 Results . . . . . . . . . . . . . . . . . . 48

3.6 Summary . . . . . . . . . . . . . . . . . . 62

4 The Bestest Little Higgs Model $\quad 64$

4.1 Introduction . . . . . . . . . . . . . . . 64

4.2 Scalar Sector and Electroweak Symmetry Breaking . . . . . . . 66

4.3 Gauge Sector . . . . . . . . . . . . . . . 75

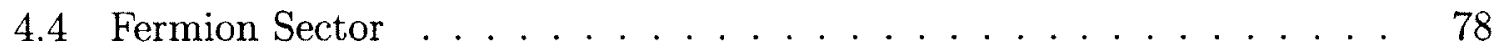

4.5 Radiative Corrections to the Scalar Potential and Fine-Tuning . . . . 85

4.6 Summary . . . . . . . . . . . . . . . . 88

5 Heavy Quark Production in the Bestest Little Higgs Model at the $\begin{array}{ll}\text { Large Hadron Collider } & 90\end{array}$ 


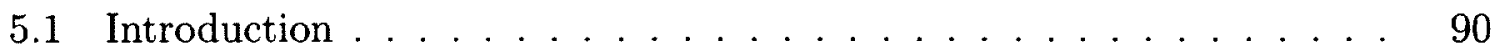

5.2 Parameters of the Bestest Little Higgs Model . . . . . . . . . . . . 91

5.3 Pair Production and Discovery Limits of Charge 2/3 Heavy Quarks . 102

5.4 Single Production of Charge 2/3 Heavy Quarks . . . . . . . . . 109

5.5 Single Associated Production of the Charge 5/3 Heavy Quark, $T_{b}^{(5 / 3)} \quad 115$

5.5 .1 Introduction . . . . . . . . . . . . . . . 115

5.5 .2 Signal and Backgrounds $\ldots \ldots \ldots \ldots \ldots$

5.5 .3 Calculations . . . . . . . . . . . . . . . . . . . . 119

5.5 .4 Results. . . . . . . . . . . . . . . . . 124

5.6 Summary ........................ 133

6 Conclusions $\quad 135$

$\begin{array}{ll}\text { Appendix A Notations and Conventions } & 139\end{array}$

$\begin{array}{ll}\text { Appendix B The Bestest Little Higgs Model } & 141\end{array}$

B.1 Group Theory Results . . . . . . . . . . . . . . . . . . . . . . . . . 141

B.2 Scalar Mass Eigenstates . . . . . . . . . . . . . . . . 142

B.3 Gauge Boson Mass Eigenstates _. . . . . . . . . . . . . . 145

B.4 Charge 2/3 Heavy Quark Mass Eigenstates . . . . . . . . . . 148

B.5 Calculation of Feynman Rules . . . . . . . . . . . 156

$\begin{array}{lr}\text { References } & 169\end{array}$ 


\section{List of Tables}

2.1 The known fundamental particles of the Standard Model and their

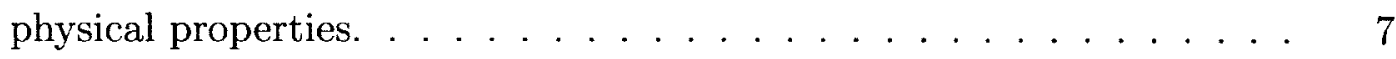

2.2 Quantum numbers of the Standard Model particles. . . . . . . . . . 9

3.1 Feynman rules for the interactions of scalar and vector bosons in the Littlest Higgs, Georgi-Machacek and Left-Right Symmetric models. .

3.2 Leptonic cuts and jet cuts used in vector boson scattering to enhance the signal to background ratio. The central jet veto cuts are interpreted as: "Reject all events with jets having $|y|<y_{\max }$ and $p_{T}>p_{T_{\min }} . "$.

3.3 The partial widths of the Higgs triplet bosons for the models under study. The widths are in GeV. . . . . . . . . . . . . . . . .

3.4 LHC cross sections (in $\mathrm{fb}$ ) for $W^{ \pm} W^{ \pm}$scattering with leptonic decays using the cuts listed in Table 3.2. The cross sections are shown for the three models under study, using a triplet vev of $v^{\prime}=39 \mathrm{GeV}$ and Higgs triplet masses of $M_{\Phi}=1.0 \mathrm{TeV}$ and $M_{\Phi}=1.5 \mathrm{TeV}$. The cross section values from MadGraph have statistical uncertainties of $\sim 1 \%$. . .

3.5 LHC cross sections (in $\mathrm{fb}$ ) for $W^{ \pm} Z$ scattering with leptonic decays using the cuts listed in Table 3.2. The cross sections are shown for the three models under study, using a triplet vev of $v^{\prime}=39 \mathrm{GeV}$ and Higgs triplet masses of $M_{\Phi}=1.0 \mathrm{TeV}$ and $M_{\Phi}=1.5 \mathrm{TeV}$. The cross section values from MadGraph have statistical uncertainties of $\sim 1 \%$. . . 
3.6 LHC cross sections (in $\mathrm{fb}$ ) for $W^{+} W^{-}$scattering with leptonic decays using the cuts listed in Table 3.2. The cross sections are shown for the three models under study, using a triplet vev of $v^{t}=39 \mathrm{GeV}$ and Higgs triplet masses of $M_{\Phi}=1.0 \mathrm{TeV}$ and $M_{\Phi}=1.5 \mathrm{TeV}$. The cross section values from MadGraph have statistical uncertainties of $\sim 1 \%$. Note that the $W^{+} W^{-}$scattering signal does not occur in the Littlest Higgs model. . . . . . . . . . . . . . . . . . . .

3.7 LHC cross sections (in $\mathrm{fb}$ ) for $Z Z$ scattering with decays to four leptons using the cuts listed in Table 3.2. The cross sections are shown for the three models under study, using a triplet vev of $v^{\prime}=39 \mathrm{GeV}$ and Higgs triplet masses of $M_{\Phi}=1.0 \mathrm{TeV}$ and $M_{\Phi}=1.5 \mathrm{TeV}$. The cross section values from MadGraph have statistical uncertainties of $\sim 1 \%$. Note that a central jet veto was not needed in the $Z Z$ channel as this was found to reduce the already small number of signal events. . . . . . 60

3.8 The integrated luminosity (in $\mathrm{fb}^{-1}$ ) required for a statistical significance of $S / \sqrt{B} \geq 5$ and at least 10 signal events, after the cuts of Table 3.2 have been imposed. These results were obtained for a triplet vev of $v^{\prime}=39 \mathrm{GeV}$, using the cross section values listed in Tables

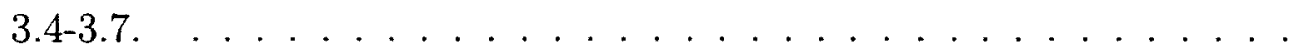

4.1 Quantum numbers for the fermions in the Bestest Little Higgs model.

5.1 Values of the $A, B, C, D, E$ parameters of Equation 5.38 for heavy quark pair production at the LHC for $\sqrt{s}=7 \mathrm{TeV}$. The values are evaluated at the scale $\mu=M_{T_{i}}$ using the CTEQ6.6M PDF set. . . . 103 
5.2 Summary of charge $2 / 3$ heavy quark pair and single production cross sections times branching ratios, assuming decays to the $b W$ final state for an LHC centre of mass energy of $\sqrt{s}=7 \mathrm{TeV}$. The single production results listed here are explained in Section 5.4 . . . . . . . . 105

5.3 Summary of charge $2 / 3$ heavy quark pair and single production cross sections times branching ratios, assuming decays to the $t Z$ final state for an LHC centre of mass energy of $\sqrt{s}=7 \mathrm{TeV}$. The single production results listed here are explained in Section 5.4 . . . . . . . . . 106

5.4 Values of the $A, B, C$ parameters of Equation 5.43 for heavy quark single production processes at the LHC for $\sqrt{s}=7 \mathrm{TeV}$. The values are evaluated at the scale $\mu=M_{T_{i}}$ using the CTEQ6.6M PDF set. . . 110

5.5 Lepton charge misidentification probability for various ranges of $p_{T}$. . 122

5.6 Cuts used for $T_{b}^{(5 / 3)}$ single associated production to enhance the signal to background ratio in the same-sign dilepton channel. . . . . . . . 124

5.7 LHC cross sections (in $\mathrm{fb}$ ) for $T_{b}^{(5 / 3)}$ single associated production in the same-sign dilepton channel using the cuts listed in Table 5.6 for $M_{T_{b}^{(5 / 3)}}=400 \mathrm{GeV}$ in the isolated and non-isolated scenarios. The cross sections for both the $\ell^{+} \ell^{+}$and $\ell^{-} \ell^{-}$channels are listed. The cross section values obtained using MadGraph5 have statistical uncertainties

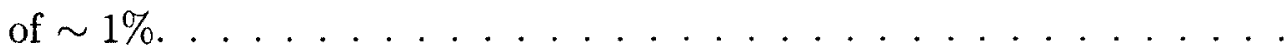

5.8 LHC cross sections (in $\mathrm{fb}$ ) for $T_{b}^{(5 / 3)}$ single associated production in the same-sign dilepton channel using the cuts listed in Table 5.6 for $M_{T_{b}^{(5 / 3)}}=600 \mathrm{GeV}$ in the isolated and non-isolated scenarios. The cross sections for both the $\ell^{+} \ell^{+}$and $\ell^{-} \ell^{-}$channels are listed. The cross section values obtained using MadGraph5 have statistical uncertainties of $\sim 1 \% \ldots \ldots \ldots \ldots \ldots \ldots \ldots \ldots$ 
5.9 LHC cross sections (in fb) for $T_{b}^{(5 / 3)}$ single associated production in the same-sign dilepton channel using the cuts listed in Table 5.6 for $M_{T_{b}^{(5 / 3)}}=800 \mathrm{GeV}$ in the isolated and non-isolated scenarios. The cross sections for both the $\ell^{+} \ell^{+}$and $\ell^{-} \ell^{-}$channels are listed. The cross section values obtained using MadGraph5 have statistical uncertainties

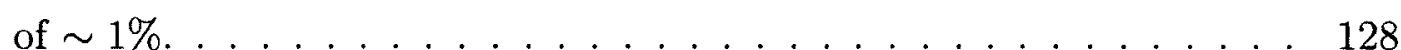

5.10 Discovery potential of the $T_{b}^{(5 / 3)}$ heavy quark for both the sum and difference of the $\ell^{+} \ell^{+}$and $\ell^{-} \ell^{-}$channels. The signal and background cross sections (in fb), calculated from the values in Tables 5.7-5.9, are listed for each scenario considered. Also listed are the integrated luminosities (in $\mathrm{fb}^{-1}$ ) required for a statistical significance of $S / \sqrt{B} \geq 5$ and at least 10 signal events in each scenario, after the cuts of Table 5.6 have been imposed. . . . . . . . . . . . 


\section{List of Figures}

2.1 Divergent loop contributions to the Higgs boson mass . . . . . . . . .

3.1 Higgs triplet production via vector boson scattering at the LHC. The incoming quarks from the colliding protons radiate vector bosons, which interact to produce a Higgs triplet as an s-channel resonance. Two vector bosons emerge in the final state, along with two forward spectator quark jets. . . . . . . . . . . . . .

3.2 The transverse mass distributions for the $W^{ \pm} Z$ signals of the Littlest Higgs (LH), Georgi-Machacek (GM) and Left-Right Symmetric (LR) models, along with the backgrounds, before and after imposing the cuts of Table 3.2. A Higgs triplet mass of $M_{\Phi}=1.0 \mathrm{TeV}$ was used, along with a triplet vev of $v^{\prime}=39 \mathrm{GeV}$, assuming an integrated luminosity of $\mathcal{L}=100 \mathrm{fb}^{-1}$ at $\sqrt{s}=14 \mathrm{TeV}$. Note that the backgrounds are stacked, whereas the signal lines are not. It can be seen that the backgrounds are reduced and a relatively large fraction of the signal events remain. The horizontal scale was enlarged in the bottom figure to more clearly show the differences in the signal lines of each model after imposing cuts. . . . . . . . . . . . . . . . . . . 
3.3 The transverse mass distributions for $W^{ \pm} W^{ \pm}$scattering in the Littlest Higgs (LH), Georgi-Machacek (GM) and Left-Right Symmetric (LR) models, along with the backgrounds, after imposing the cuts of Table 3.2. Higgs triplet masses of $M_{\Phi}=1.0$ and $1.5 \mathrm{TeV}$ were used, along with a triplet vev of $v^{\prime}=39 \mathrm{GeV}$, assuming an integrated luminosity of $\mathcal{L}=100 \mathrm{fb}^{-1}$ at $\sqrt{s}=14 \mathrm{TeV}$. It should be noted that the backgrounds are stacked, whereas the signal lines are not. . . . . . . .

3.4 The transverse mass distributions for $W^{ \pm} Z$ scattering in the Littlest Higgs (LH), Georgi-Machacek (GM) and Left-Right Symmetric (LR) models, along with the backgrounds, after imposing the cuts of Table 3.2. Higgs triplet masses of $M_{\Phi}=1.0$ and $1.5 \mathrm{TeV}$ were used, along with a triplet vev of $v^{\prime}=39 \mathrm{GeV}$, assuming an integrated luminosity of $\mathcal{L}=100 \mathrm{fb}^{-1}$ at $\sqrt{s}=14 \mathrm{TeV}$. It should be noted that the backgrounds are stacked, whereas the signal lines are not. . . . . . . .

3.5 The transverse mass distributions for $W^{+} W^{-}$scattering in the GeorgiMachacek (GM) and Left-Right Symmetric (LR) models (this signal does not exist in the Littlest Higgs model at lowest order), along with the backgrounds, after imposing the cuts of Table 3.2. Higgs triplet masses of $M_{\Phi}=1.0$ and $1.5 \mathrm{TeV}$ were used, along with a triplet vev of $v^{\prime}=39 \mathrm{GeV}$, assuming an integrated luminosity of $\mathcal{L}=100 \mathrm{fb}^{-1}$ at $\sqrt{s}=14 \mathrm{TeV}$. It should be noted that the backgrounds are stacked, whereas the signal lines are not. . . . . . . . . . . . . . 
3.6 The invariant mass distributions for $Z Z$ scattering in the Littlest Higgs (LH), Georgi-Machacek (GM) and Left-Right Symmetric (LR) models, along with the backgrounds, after imposing the cuts of Table 3.2. Higgs triplet masses of $M_{\Phi}=1.0$ and $1.5 \mathrm{TeV}$ were used, along with a triplet vev of $v^{\prime}=39 \mathrm{GeV}$, assuming an integrated luminosity of $\mathcal{L}=100 \mathrm{fb}^{-1}$ at $\sqrt{s}=14 \mathrm{TeV}$. It should be noted that the backgrounds are stacked, whereas the signal lines are not. The efficiency for reconstructing four leptons is expected to be $\sim 95 \%$, but this was not taken into account in this analysis.

4.1 The modular structure of the symmetry breaking in the Bestest Little Higgs model. $\Sigma$ breaks a global $S O(6)_{A} \times S O(6)_{B}$ symmetry down to the diagonal at a scale $f$, while $\Delta$ breaks a global $S U(2)_{C} \times S U(2)_{D}$ symmetry down to the diagonal at a scale $F>f$. The $S U(2)_{L A}$ symmetry in $S O(6)_{A}$ and the $S U(2)_{C}$ symmetry are gauged with the same $S U(2)_{A}$ gauge bosons. Similarly, the $S U(2)_{L B}$ symmetry in $S O(6)_{B}$ and the $S U(2)_{D}$ symmetry are gauged with the same $S U(2)_{B}$ gauge bosons. $S U(2)_{A, B}$ are subgroups of $S O(6)_{A, B}$, whose diagonal subgroup is the Standard Model $S U(2)_{L} \ldots \ldots \ldots \ldots \ldots$

5.1 Fine-tuning contours from fermion loops, $\Psi_{F}$, as a function of $\tan \theta_{12}$ and $\tan \theta_{13}$ for $f=700 \mathrm{GeV}$, showing placement of the isolated and non-isolated scenarios. . . . . . . . . . . . . . . .

5.2 Fine-tuning contours from fermion loops, $\Psi_{F}$, as a function of $\tan \theta_{12}$ and $\tan \theta_{13}$ for $f=1100 \mathrm{GeV}$, showing placement of the isolated and non-isolated scenarios. . . . . . . . . . . . . . . . 
5.3 Fine-tuning contours from fermion loops, $\Psi_{F}$, as a function of $\tan \theta_{12}$ and $\tan \theta_{13}$ for $f=1500 \mathrm{GeV}$, showing placement of the isolated and non-isolated scenarios. . . . . . . . . . . . . . . . . . . .

5.4 Fine-tuning contours from fermion loops, $\Psi_{F}$, as a function of $\tan \theta_{12}$ and $\tan \theta_{13}$ for $f=1900 \mathrm{GeV}$, showing placement of the isolated and non-isolated scenarios. . . . . . . . . . . . . . . .

$5.5 T_{b}^{(5 / 3)}$ branching ratios for all two-body final states with $B R>0.1 \%$. Branching ratios in the isolated (non-isolated) scenario are shown in the plot on the left (right) . . . . . . . . . . . . . .

$5.6 T_{b}^{(2 / 3)}$ branching ratios for all two-body final states with $B R>0.1 \%$. Branching ratios in the isolated (non-isolated) scenario are shown in the plot on the left (right). Note that since the $T_{b}^{(2 / 3)}$ state is among the lightest heavy top quarks, it cannot decay to the other top partners, resulting in similar decay modes for the isolated and non-isolated scenarios. . . . . . . . . . . . . . . .

5.7 $T_{6}$ branching ratios for all two-body final states with $B R>0.1 \%$. Branching ratios in the isolated (non-isolated) scenario are shown in the plot on the left (right). Note that since the $T_{6}$ state is among the lightest heavy top quarks, it cannot decay to the other top partners, resulting in similar decay modes for the isolated and non-isolated scenarios. . . . . . . . . . . . . . . . . 100

$5.8 T_{5}$ branching ratios for all two-body final states with $B R>0.1 \%$. Branching ratios in the isolated (non-isolated) scenario are shown in the plot on the left (right) . . . . . . . . . . . . . . 100 
$5.9 T_{a}^{d}$ branching ratios for all two-body final states with $B R>0.1 \%$. Branching ratios in the isolated (non-isolated) scenario are shown in the plot on the left (right) . . . . . . . . . . . . . . . 101

$5.10 T_{a}^{u}$ branching ratios for all two-body final states with $B R>0.1 \%$. Branching ratios in the isolated (non-isolated) scenario are shown in the plot on the left (right) . . . . . . . . . . . . . 10

5.11 Discovery limits on the lightest top partners for the isolated and nonisolated scenarios in the $b W$ final state for an LHC centre of mass energy of $\sqrt{s}=7 \mathrm{TeV}$. This plot shows the expected NLO cross section times branching ratio and the CMS $95 \%$ confidence level upper limit in the $b W$ final state. The point at which these curves intersect yields $95 \%$ confidence level lower limits on the mass of the lightest top partner of $369 \mathrm{GeV}$ in the isolated scenario and $419 \mathrm{GeV}$ in the non-isolated

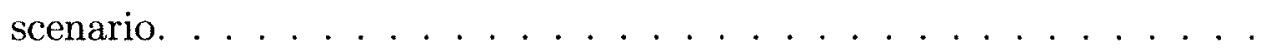

5.12 Discovery limits on the lightest top partners for the isolated and nonisolated scenarios in the $t Z$ final state for an LHC centre of mass energy of $\sqrt{s}=7 \mathrm{TeV}$. This plot shows the expected NLO cross section times branching ratio and the CMS $95 \%$ confidence level upper limit in the $t Z$ final state. The point at which these curves intersect yields $95 \%$ confidence level lower limits on the mass of the lightest top partner of $392 \mathrm{GeV}$ in the isolated scenario and $361 \mathrm{GeV}$ in the non-isolated scenario. . . . . . . . . . . . . . . . . . . . 108

5.13 Combined single production NLO cross sections times branching ratios in the isolated scenario for $p p \rightarrow q b \rightarrow q^{\prime} T_{i}$ (black) and $p p \rightarrow q \bar{q}^{\prime} \rightarrow \bar{b} T_{i}$ (grey), for the $b W$ (solid) and $t Z$ (dashed) decay channels at $\sqrt{s}=$ $7 \mathrm{TeV}$. Cross sections include the charge conjugate processes. 
5.14 Combined single production NLO cross sections times branching ratios in the non-isolated scenario for $p p \rightarrow q b \rightarrow q^{\prime} T_{i}$ (black) and $p p \rightarrow$ $q \bar{q}^{\prime} \rightarrow \bar{b} T_{i}$ (grey), for the $b W$ (solid) and $t Z$ (dashed) decay channels at $\sqrt{s}=7 \mathrm{TeV}$. Cross sections include the charge conjugate processes. 113

5.15 Ratio of NLO and LO cross sections for single production via t-channel $W$ exchange for $\sqrt{s}=7 \mathrm{TeV}$, as a function of the top partner mass. Only one curve is shown as the $\mathrm{K}$-factors were found to be identical for every scenario. . . . . . . . . . . . . . . . . . . . 114

5.16 Production of the exotic charge $5 / 3$ heavy quark, $T_{b}^{(5 / 3)}$, in association with a $\bar{t}$ quark and a light quark jet, with decays to same-sign dileptons in the final state. . . . . . . . . . . . . . . . . 117

5.17 Sum of the $T_{b}^{(5 / 3)}$ and $\bar{T}_{b}^{(5 / 3)}$ production cross sections with decays to same-sign dileptons for both the isolated and non-isolated scenarios, at LHC centre of mass energies of $\sqrt{s}=7 \mathrm{TeV}, 8 \mathrm{TeV}$ and $14 \mathrm{TeV}$. Discovery of the $T_{b}^{(5 / 3)}$ heavy quark will be very difficult at $\sqrt{s}=$ $7 \mathrm{TeV}$ and $8 \mathrm{TeV}$ due to the relatively small number of signal events. Therefore, the $T_{b}^{(5 / 3)}$ production cross sections were calculated at the full LHC centre of mass energy of $\sqrt{s}=14 \mathrm{TeV}$, which increases the signal cross section by roughly an order of magnitude. . . . . . . .

$5.18 H_{T}$ distributions for $T_{b}^{(5 / 3)}$ production signals and backgrounds, based on the cross sections of Table 5.7 for $M_{T_{b}^{(5 / 3)}}=400 \mathrm{GeV}$ in the isolated and non-isolated scenarios. This plot shows the sum, $\frac{d \sigma}{d H_{T}}\left(\ell^{+} \ell^{+}\right)+$ $\frac{d \sigma}{d H_{T}}\left(\ell^{-} \ell^{-}\right)$, of the two same-sign dilepton channels. The backgrounds are stacked, and the two signals are shown as a broad enhancement above the background. . . . . . . . . . . . . 
$5.19 H_{T}$ distributions for $T_{b}^{(5 / 3)}$ production signals and backgrounds, based on the cross sections of Table 5.7 for $M_{T_{b}^{(5 / 3)}}=400 \mathrm{GeV}$ in the isolated and non-isolated scenarios. This plot shows the difference, $\frac{d \sigma}{d H_{T}}\left(\ell^{+} \ell^{+}\right)-$ $\frac{d \sigma}{d H_{T}}\left(\ell^{-} \ell^{-}\right)$, of the two same-sign dilepton channels. The backgrounds are stacked, and the two signals are shown as a broad enhancement above the background. . . . . . . . . . . . . 130 


\section{Chapter 1}

\section{Introduction}

Current knowledge of particle physics is described by the Standard Model, a theory that governs the interactions between the known fundamental particles. The Standard Model predicts the results of most experiments with impressive accuracy [1]. However, despite the success of the Standard Model, the general consensus among particle physicists is that the Standard Model is incomplete, offering merely a lowenergy description of a more fundamental theory. One of the reasons for this concern is related to Electroweak Symmetry Breaking (EWSB), the mechanism by which particles acquire mass. In the Standard Model, EWSB occurs via the Higgs mechanism, which predicts the existence of a particle called the Higgs boson, with a mass on the order of the weak scale $(v \approx 246 \mathrm{GeV})$. However, ever since the Higgs mechanism was proposed in 1964 [2], the Higgs boson had yet to be discovered experimentally. Its discovery by the ATLAS [3] and CMS [4] experiments at the Large Hadron Collider (LHC) in July 2012 was marked with a great deal of excitement among the particle physics community, as this provided evidence for the final missing piece of the Standard Model, without which there is no explanation for the origin of the masses of the Standard Model particles. Furthermore, the Higgs boson was found to be relatively light with a mass of approximately $125 \mathrm{GeV}$. As described below, this suggests 
that the Higgs mechanism is not entirely responsible for EWSB and that the Standard Model is simply a low-energy manifestation of a more fundamental theory, the particles and interactions of which may also be discovered at the LHC. Therefore, discovering the Higgs boson is a crucial step towards understanding the mechanism responsible for EWSB. The details of the Higgs boson discovery are described in greater detail in Section 2.5.

Unfortunately, higher-order corrections to the Higgs boson mass become disastrously large if the Standard Model is valid up to the Planck scale $\left(M_{P l a n c k} \approx\right.$ $10^{19} \mathrm{GeV}$ ), the scale at which the strength of gravity becomes comparable to the other forces. This is known as the hierarchy problem. The existence of some type of new physics above the weak scale, but below the Planck scale, is required to cancel these divergent corrections without introducing a large, unnatural fine-tuning to the Higgs boson mass. Most theories of physics beyond the Standard Model predict the existence of new particles and interactions at the TeV scale. However, as energies at the $\mathrm{TeV}$ scale have yet to be probed by particle physics experiments, it is currently unknown what type of particles and interactions exist beyond the Standard Model.

Although a considerable number of models have been proposed to offer a more self-consistent description of EWSB up to the TeV scale, only experimental data will reveal which one, if any, is correct. This is the ultimate goal of experiments at the LHC, a particle accelerator located at the CERN laboratory near Geneva, Switzerland. As its principal detectors, ATLAS and CMS, collect data, large regions of parameter space are being ruled out in many models of beyond the Standard Model physics. For example, the parameter space of Supersymmetric models is shrinking rapidly leading to an increasing need for fine-tuning [5-8]. This comparison of theoretical predictions and experimental results is the foundation of an area of particle physics known as phenomenology. 
The Tevatron at the Fermilab National Laboratory in Batavia, Illinois, pushed the high-energy frontier by colliding protons and anti-protons at a centre of mass energy of $1.8 \mathrm{TeV}$ beginning in 1986 . Tevatron Run II increased the centre of mass energy to $1.96 \mathrm{TeV}$ from 2001 until the experiment ceased operations in 2011. This facility was instrumental in improving understanding of the Standard Model, particularly with its discovery of the top quark in 1995 [9]. In 2010, the LHC surpassed the Tevatron as the world's highest energy particle accelerator, as it began colliding protons with a centre of mass energy of $7 \mathrm{TeV}$. As of this writing, the LHC is running at $8 \mathrm{TeV}$ until the end of 2012, with plans to collide protons at the full design centre of mass energy of $14 \mathrm{TeV}$ by 2014. Due to its high centre of mass energy, the LHC is known as a "discovery machine", as it is capable of producing $\mathrm{TeV}$ scale particles for the first time under laboratory conditions. More precise measurements can be performed at a lepton collider, such as the proposed International Linear Collider (ILC), which plans to collide electrons and positrons to more precisely measure the properties of any particles discovered at the LHC.

The year 2012 marks an exciting time for the particle physics community, as the ATLAS and CMS experiments at the LHC are beginning to acquire enough data to study physics at the $\mathrm{TeV}$ energy scale at which new physics are expected to arise. The goal of the LHC is to discover (or rule out) the Standard Model Higgs boson and to determine what lies beyond the Standard Model, in order to gain an improved understanding of EWSB [10-12]. The long term goal of the work in this thesis is to compare the predictions of various models so that once enough data from the LHC becomes available, these models can be tested experimentally and the correct mechanism for EWSB can be determined.

In Chapter 2, the Standard Model is introduced, with emphasis on the electroweak sector of the theory. The structure of a relatively recent class of models, called 
Little Higgs models [13-16], is then introduced as a possible solution to the hierarchy problem. Little Higgs models predict the existence of several new particles at the $\mathrm{TeV}$ scale. In particular, the production and discovery potential of a triplet of heavy Higgs bosons at the LHC is discussed in Chapter 3 in the context of the Littlest Higgs model [17], as well as in two other Higgs triplet models, the Georgi-Machacek model [18], and the Left-Right Symmetric model [19,20]. In Chapter 4, the Bestest Little Higgs model [21], which was formulated to solve the theoretical inconsistencies present in most other Little Higgs models, is discussed in detail. The Bestest Little Higgs model predicts the existence of several heavy top quark partners, which can be readily produced at the LHC. Therefore, the production and discovery potential of these heavy top quark partners at the LHC is the focus of Chapter 5. Finally, the results of this thesis are summarized and discussed in Chapter 6 . 


\section{Chapter 2}

\section{The Standard Model}

\section{$2.1 \quad$ Introduction}

According to current knowledge of physics, there are four fundamental interactions that exist in nature: gravitation, electromagnetism, the weak interaction, and the strong interaction. Gravitation, described classically by the general theory of relativity (a satisfactory theory of quantum gravity has not yet been formulated), is the weakest of the four interactions and is assumed to play a negligible role in elementary particle physics. The electromagnetic (EM) interaction is best described by the theory of Quantum Electrodynamics (QED) and proceeds by the exchange of massless spin-one photons (denoted as $\gamma$ ) between charged particles. Weak interactions (first discovered in $\beta$-decay) are mediated by the massive spin-one $W^{ \pm}$and $Z^{0}$ gauge bosons. Following the work of Glashow, Weinberg and Salam [22-24], the theories for the electromagnetic and weak interactions can be described by a single theory of electroweak interactions. The strong interaction, described by the theory of Quantum Chromodynamics (QCD), describes the interactions between quarks, which proceed by the exchange of massless spin-one gluons $[25,26]$. 
The Standard Model of particle physics is a theory that encompasses the electromagnetic, weak and strong interactions ${ }^{1}$. Particles in the Standard Model can be classified into two groups based on their spin: fermions (i.e. quarks and leptons) have half-integer spin, and bosons (i.e. $\gamma, W^{ \pm}, Z^{0}$ and the gluons) have integer spin. The important physical properties of the known Standard Model particles are summarized in Table 2.1 [1]. One should note that each fermion in this table has a corresponding anti-particle with a charge of equal magnitude and opposite sign. One should also note that although all three generations of fermions have been observed experimentally, only the first generation, $(u, d)$ and $\left(e, \nu_{e}\right)$, is found in normal matter. Fermions of the second and third generations are more massive and unstable, decaying to particles of the first generation.

The Standard Model group structure is $S U(3)_{c} \times S U(2)_{L} \times U(1)_{Y}$, where $c$ refers to the colour charge of $\mathrm{QCD}, L$ refers to a left-handed group structure, and $Y$ is the hypercharge group. The helicity of a particle is defined as follows: an observer in an inertial reference frame says that a particle is left-handed (right-handed) if its spin is oriented opposite (along) its direction of motion, as measured from that particular frame. While all quarks and leptons (which appear as both left-handed doublets and right-handed singlets) transform non-trivially under $U(1)_{Y}$, only the left-handed fermion doublets transform non-trivially under $S U(2)_{L}$, and only the quarks and gluons carry the colour charge of QCD.

In this chapter, the mathematical structure of the Standard Model is described, by outlining the QCD Lagrangian in Section 2.2 and the Electroweak Lagrangian in Section 2.3. The $S U(2)_{L} \times U(1)_{Y}$ theory of electroweak interactions predicts the existence of an additional spin-zero (scalar) particle called the Higgs boson. The interactions of the Standard Model particles with the Higgs boson are believed to

\footnotetext{
${ }^{1}$ The theoretical framework of the Standard Model is outlined in several textbooks and reviews. See, for example, [27-30].
} 
Table 2.1: The known fundamental particles of the Standard Model and their physical properties [1].

\begin{tabular}{|c|c|c|}
\hline Quarks $\left(\operatorname{spin} \frac{1}{2}\right)$ & Mass & Electric Charge (e) \\
\hline up quark $(u)$ & $1.7-3.3 \mathrm{MeV}$ & \\
\hline charm quark $(c)$ & $1.27_{-0.09}^{+0.07} \mathrm{GeV}$ & $+\frac{2}{3}$ \\
\hline top quark $(t)$ & $172.0 \pm 1.6 \mathrm{GeV}$ & \\
\hline down quark $(d)$ & 4.1-5.8 MeV & \\
\hline strange quark $(s)$ & $101_{-21}^{+29} \mathrm{MeV}$ & $-\frac{1}{3}$ \\
\hline bottom quark $(b)$ & $4.19_{-0.06}^{+0.18} \mathrm{GeV}$ & \\
\hline Leptons $\left(\operatorname{spin} \frac{1}{2}\right)$ & Mass & Electric Charge $(e)$ \\
\hline electron $\left(e^{-}\right)$ & $0.511 \mathrm{MeV}$ & \\
\hline muon $\left(\mu^{-}\right)$ & $105.66 \mathrm{MeV}$ & -1 \\
\hline $\operatorname{tau}\left(\tau^{-}\right)$ & $1776.82 \pm 0.16 \mathrm{MeV}$ & \\
\hline electron neutrino $\left(\nu_{e}\right)$ & $<2 \mathrm{eV}$ & \\
\hline muon neutrino $\left(\nu_{\mu}\right)$ & $<0.19 \mathrm{MeV}$ & 0 \\
\hline tau neutrino $\left(\nu_{\tau}\right)$ & $<18.2 \mathrm{MeV}$ & \\
\hline Bosons (spin 1) & Mass & Electric Charge $(e)$ \\
\hline gluon $(g)$ & $0^{*}$ & 0 \\
\hline photon $(\gamma)$ & $<1 \times 10^{-18} \mathrm{eV}$ & 0 \\
\hline$Z$ boson $\left(Z^{0}\right)$ & $91.1876 \pm 0.0021 \mathrm{GeV}$ & 0 \\
\hline$W$ boson $\left(W^{ \pm}\right)$ & $80.399 \pm 0.023 \mathrm{GeV}$ & \pm 1 \\
\hline
\end{tabular}

* Theoretical value. A mass as large as a few MeV may not be precluded. 
be responsible for generating their masses via the Higgs mechanism, which will be described in Section 2.4. Limits on the mass of the Higgs boson are presented in Section 2.5. The major theoretical difficulties with the Standard Model, particularly the hierarchy problem, are discussed in Section 2.6, and a possible solution to these problems, Little Higgs models, is introduced in Section 2.7.

\subsection{Quantum Chromodynamics (QCD)}

The strong interactions of the Standard Model are described by Quantum Chromodynamics (QCD) $[25,26]$, which is an $S U(3)_{c}$ gauge theory. The interactions of this theory are described by the Lagrangian density [27]

$$
\mathcal{L}_{Q C D}=-\frac{1}{4} F_{\mu \nu}^{i} F^{i \mu \nu}+\sum_{r} \bar{q}_{r \alpha} i \not_{\beta}^{\alpha} q_{r}^{\beta}
$$

where

$$
F_{\mu \nu}^{i}=\partial_{\mu} G_{\nu}^{i}-\partial_{\nu} G_{\mu}^{i}-g_{s} f_{i j k} G_{\mu}^{j} G_{\nu}^{k}
$$

is the field strength tensor for the gluon fields, $G_{\mu}^{i}(i=1 \ldots 8)$, and $f_{i j k}$ are the totally antisymmetric structure constants of $S U(3)$. The last term of Equation 2.2, when substituted into the first term of Equation 2.1, gives rise to the three- and four-point gluon self interactions. These gluon self interactions give rise to the property of QCD known as asymptotic freedom, in which the value of the strong coupling, $g_{s}$, varies (or "runs") with energy. This running coupling becomes weak at high energies or short distances. Meanwhile, at low energies or large distances, the theory becomes strongly coupled, resulting in the confinement of quarks and gluons into bound states known as hadrons.

The second term of Equation 2.1 leads to the interactions of the gluons with the three generations $(r=1,2,3)$ of quark doublets, $q_{r}^{\beta}$, and antiquark doublets, 
Table 2.2: Quantum numbers of the Standard Model particles.

\begin{tabular}{|c|c|c|c|}
\hline Field $(r=1,2,3)$ & $S U(3)_{c}$ & $S U(2)_{L}$ & $U(1)_{Y}$ \\
\hline$q_{r L}$ & $\mathbf{3}$ & $\mathbf{2}$ & $+\frac{1}{6}$ \\
$\ell_{r L}$ & $\mathbf{1}$ & $\mathbf{2}$ & $-\frac{1}{2}$ \\
$u_{r R}$ & $\mathbf{3}$ & $\mathbf{1}$ & $+\frac{2}{3}$ \\
$d_{r R}$ & $\mathbf{3}$ & $\mathbf{1}$ & $-\frac{1}{3}$ \\
$e_{r R}^{-}$ & $\mathbf{1}$ & $\mathbf{1}$ & -1 \\
$g$ & $\mathbf{8}$ & $\mathbf{1}$ & 0 \\
$W$ & $\mathbf{1}$ & $\mathbf{3}$ & 0 \\
$B$ & $\mathbf{1}$ & $\mathbf{1}$ & 0 \\
$\Phi$ & $\mathbf{1}$ & $\mathbf{2}$ & $+\frac{1}{2}$ \\
\hline
\end{tabular}

$\bar{q}_{r \alpha}$, where $\alpha, \beta=1,2,3$ are the colour indices. The gauge covariant derivative, $\not D_{\beta}^{\alpha}=\gamma^{\mu} D_{\mu \beta}^{\alpha}$, is given by $[27]$

$$
D_{\mu \beta}^{\alpha}=\left(D_{\mu}\right)_{\alpha \beta}=\partial_{\mu} \delta_{\alpha \beta}+i g_{s} G_{\mu}^{i} L_{\alpha \beta}^{i}
$$

where $L^{i}=\lambda^{i} / 2(i=1 \ldots 8)$ are the generators of $S U(3), \delta_{\alpha \beta}$ is the Kronecker delta symbol and the Lorentz $\gamma$-matrices are defined in Appendix A.

It is important to note that there are no bare mass terms for the quarks in Equation 2.1. These are allowed by QCD alone, but are forbidden by the chiral symmetry of the electroweak theory, as described in Section 2.3. The Standard Model quark masses are instead generated by the Higgs mechanism [2], as explained in Section 2.4. 


\subsection{The Electroweak Theory}

The Glashow-Weinberg-Salam theory of electroweak interactions [22-24] is an

$S U(2)_{L} \times U(1)_{Y}$ gauge theory containing three $S U(2)_{L}$ gauge bosons, $W_{\mu}^{i}(i=1,2,3)$, with coupling constant $g$, and one $U(1)_{Y}$ gauge boson, $B_{\mu}$, with coupling constant $g^{\prime}$. The masses and interactions of the electroweak theory are described by the Lagrangian density [27]

$$
\mathcal{L}_{E W}=\mathcal{L}_{\text {gauge }}+\mathcal{L}_{\phi}+\mathcal{L}_{f}+\mathcal{L}_{Y u k}
$$

The gauge kinetic energy terms of the electroweak Lagrangian are given by

$$
\mathcal{L}_{\text {gauge }}=-\frac{1}{4} W_{\mu \nu}^{i} W^{\mu \nu i}-\frac{1}{4} B_{\mu \nu} B^{\mu \nu}
$$

where

$$
\begin{aligned}
B_{\mu \nu} & =\partial_{\mu} B_{\nu}-\partial_{\nu} B_{\mu} \\
W_{\mu \nu}^{i} & =\partial_{\mu} W_{\nu}^{i}-\partial_{\nu} W_{\mu}^{i}-g \epsilon_{i j k} W_{\mu}^{j} W_{\nu}^{k}
\end{aligned}
$$

where $\epsilon_{i j k}$ is the totally antisymmetric tensor, defined by $\epsilon_{123}=1$, which changes sign under an odd number of permutations of the indices. The $U(1)_{Y}$ quantum number, known as hypercharge, is defined as [27]

$$
Y=Q-T_{L}^{3}
$$

where $Q$ and $T_{L}^{3}=\tau^{3} / 2$ are the electric charge operator and the third component of weak isospin, respectively. The corresponding eigenvalues of these operators are denoted by $y, q$ and $t_{L}^{3}$, respectively. 
$\mathcal{L}_{f}$ and $\mathcal{L}_{Y u k}$ describe the fermion-gauge boson and fermion-scalar (Yukawa) interactions, respectively. As a detailed description of these terms in the Standard Model is not crucial for the purposes of this thesis, interested readers may consult reviews such as [27].

The scalar part of the Lagrangian is given by $[27,28]$

$$
\mathcal{L}_{\phi}=\left(D^{\mu} \Phi\right)^{\dagger}\left(D_{\mu} \Phi\right)-V(\Phi)
$$

where the complex Higgs scalar

$$
\Phi=\left(\begin{array}{c}
\phi^{+} \\
\phi^{0}
\end{array}\right)
$$

is an $S U(2)$ doublet with hypercharge $y_{\Phi}=1 / 2$. The gauge covariant derivative is $[27,28]$

$$
D_{\mu} \Phi=\left(\partial_{\mu}+i g \frac{\tau^{i}}{2} W_{\mu}^{i}+i g^{\prime} y_{\Phi} B_{\mu}\right) \Phi
$$

where $\tau^{i}$ are the $2 \times 2$ Pauli matrices defined in Equation A.6 of Appendix A. The scalar potential, $V(\Phi)$, is given by $[27,28]$

$$
V(\Phi)=\mu^{2}\left|\Phi^{\dagger} \Phi\right|+\lambda\left|\Phi^{\dagger} \Phi\right|^{2}
$$

where $\lambda>0$ is the Higgs quartic coupling and $\mu^{2}<0$ results in Electroweak Symmetry Breaking, as described in Section 2.4. 


\subsection{Electroweak Symmetry Breaking and the Higgs Mechanism}

The Electroweak Lagrangian of Equation 2.4 is required to be gauge invariant. That is, it must be invariant under local $U(1)$ and $S U(2)$ gauge transformations of the fields of the form

$$
\begin{aligned}
B_{\mu} & \rightarrow B_{\mu}+\partial_{\mu} \alpha(x) \\
W_{\mu}^{i} & \rightarrow W_{\mu}^{i}+\partial_{\mu} \Lambda^{i}(x)+g \epsilon^{i j k} \Lambda^{j}(x) W_{\mu}^{k}
\end{aligned}
$$

If mass terms of the form $\mathcal{L}_{M_{B}}=\frac{1}{2} M_{B}^{2} B_{\mu} B^{\mu}$ and $\mathcal{L}_{M_{W}}=\frac{1}{2} M_{W}^{2} W_{\mu}^{i} W^{\mu i}$ are added to the gauge kinetic terms of Equation 2.5, then the Electroweak Lagrangian is no longer gauge invariant under the transformations of Equations 2.13 and 2.14. This suggests that $M_{B}=M_{W}=0$, implying that all four gauge bosons must be massless. However, in the electroweak theory, the photon is the only massless gauge boson; the current experimental values for the $W^{ \pm}$and $Z^{0}$ boson masses are $M_{W}=80.399 \pm 0.023 \mathrm{GeV}$ and $M_{Z}=91.1876 \pm 0.0021 \mathrm{GeV}[1]$.

Fermions can be written in terms of their left- and right-handed projections as $\psi=$ $\psi_{L}+\psi_{R}$, where $\psi_{L, R}=\frac{1}{2}\left(1 \mp \gamma_{5}\right) \psi$, with $\gamma_{5}$ defined by Equation A.7 of Appendix A. Since left- and right-handed fermions transform differently under the chiral $S U(2)_{L} \times$ $U(1)_{Y}$ gauge groups, a fermion mass term of the form

$$
\mathcal{L}_{m_{f}}=-m_{f} \bar{\psi} \psi=-m_{f}\left(\bar{\psi}_{L} \psi_{R}+\bar{\psi}_{R} \psi_{L}\right)
$$

is not gauge invariant and is therefore forbidden $[27,28]$. However, the quarks are known to have non-zero masses, as listed in Table 2.1. The masses of the $W^{ \pm}$and $Z^{0}$ bosons, as well as the quark and lepton masses, must therefore be generated by 
a different mechanism, known as Electroweak Symmetry Breaking (EWSB). In the Standard Model, EWSB proceeds by the Higgs mechanism [2].

Consider the Higgs potential of Equation 2.12. If $\mu^{2}<0$, then $V(\Phi)$ is such that the state of minimum energy is not at $\Phi=0$ (as would be the case for $\mu^{2}>0$ ). By convention, the vacuum expectation value (vev) of $\Phi$ is chosen to correspond to the state of minimum energy, given by $[2,27,28]$

$$
\langle\Phi\rangle=\langle 0|\Phi| 0\rangle=\frac{1}{\sqrt{2}}\left(\begin{array}{l}
0 \\
v
\end{array}\right),
$$

where minimizing the Higgs potential of Equation 2.12 leads to the relation $v=$ $\sqrt{-\mu^{2} / \lambda}$. As a particular orientation for the minimum has been chosen, it is said that the electroweak symmetry has been broken. More precisely, the generators $\frac{\tau^{1}}{2}$, $\frac{\tau^{2}}{2}$ and $\frac{\tau^{3}}{2}-Y$ are spontaneously broken, since, for example, $\frac{\tau^{1}}{2}\langle\Phi\rangle \neq 0$. On the other hand, the vacuum carries no electric charge, since $Q\langle\Phi\rangle=\left(\frac{\tau^{3}}{2}+Y\right)\langle\Phi\rangle=0$, so the electromagnetic symmetry has not been broken $[2,27,28]$. The symmetry breaking scheme of the Standard Model is therefore

$$
S U(2)_{L} \times U(1)_{Y} \rightarrow U(1)_{E M}
$$

Since $S U(2)_{L} \times U(1)_{Y}$ has four symmetry generators and $U(1)_{E M}$ has one, three of the generators of $S U(2)_{L} \times U(1)_{Y}$ have been broken. Accurding to Goldstone's theorem [31], the number of broken symmetry generators is equal to the number of massless scalars called Goldstone bosons. Therefore, in the Standard Model, there are three Goldstone bosons, $G^{i}(i=1,2,3)$.

In order to see the physical particle spectrum, the fields must be shifted about 
their vev as follows:

$$
\Phi=\frac{1}{\sqrt{2}} e^{i \frac{G^{i} \tau^{i}}{2 v}}\left(\begin{array}{c}
0 \\
v+h
\end{array}\right)
$$

where $G^{i}(i=1,2,3)$ and $h$ are four real fields with no vev $[2,27,28]$. Making a gauge transformation

$$
\Phi \rightarrow e^{-i \frac{G^{i} \tau^{i}}{2 v}} \Phi
$$

results in the unitary gauge, in which only the physical fields are present:

$$
\Phi=\frac{1}{\sqrt{2}}\left(\begin{array}{c}
0 \\
v+h
\end{array}\right)
$$

Substitution of Equation 2.20 into the scalar Lagrangian of Equation 2.9 gives

$$
\mathcal{L}_{\phi}=M_{W}^{2} W^{\mu+} W_{\mu}^{-}\left(1+\frac{h}{v}\right)^{2}+\frac{1}{2} M_{Z}^{2} Z^{\mu} Z_{\mu}\left(1+\frac{h}{v}\right)^{2}+\frac{1}{2}\left(\partial_{\mu} h\right)^{2}-V(\Phi)
$$

The physical gauge fields, $W_{\mu}^{ \pm}, Z_{\mu}$ and $A_{\mu}$, are given by $[27,28]$

$$
\begin{aligned}
W_{\mu}^{ \pm} & =\frac{1}{\sqrt{2}}\left(W_{\mu}^{1} \mp i W_{\mu}^{2}\right) \\
Z_{\mu} & =-s_{W} B_{\mu}+c_{W} W_{\mu}^{3} \\
A_{\mu} & =c_{W} B_{\mu}+s_{W} W_{\mu}^{3}
\end{aligned}
$$

where

$$
\begin{aligned}
& s_{W} \equiv \sin \theta_{W}=\frac{g^{\prime}}{\sqrt{g^{2}+g^{\prime 2}}} \\
& c_{W} \equiv \cos \theta_{W}=\frac{g}{\sqrt{g^{2}+g^{\prime 2}}} .
\end{aligned}
$$


Here, $\theta_{W}$ is the weak mixing angle and its experimental value is given by $\sin ^{2} \theta_{W}=$ $0.23116 \pm 0.00013[1]$. This also leads to a relation for the electromagnetic charge, $e$ :

$$
e=g \sin \theta_{W}=g^{\prime} \cos \theta_{W} .
$$

After the symmetry breaking, the gauge bosons obtain masses given by $[27,28]$

$$
\begin{aligned}
M_{W}^{2} & =\frac{1}{4} g^{2} v^{2} \\
M_{Z}^{2} & =\frac{1}{4}\left(g^{2}+g^{2}\right) v^{2} \\
M_{A} & =0 .
\end{aligned}
$$

As there are no mass terms in Equation 2.21 for the photon, $A_{\mu}$, it remains massless. On the other hand, through the gauge transformation of Equation 2.19, it is said that the three Goldstone bosons, $G^{i}$, have been "eaten" to give the $W^{ \pm}$and $Z$ bosons their masses. Consequently, the $W^{ \pm}$and $Z$ have acquired longitudinal polarization vectors, which become important at higher energy scales. The physical scalar field, $h$, that remains in the theory is called the Higgs boson, and this process is known as the Higgs mechanism [2].

After EWSB, the Yukawa interactions can be written in terms of the masseigenstate fermions as $[27,28]$

$$
\mathcal{L}_{Y u k}=-\sum_{i} m_{i} \bar{\psi}_{i} \psi_{i}\left(1+\frac{h}{v}\right)
$$

It is seen from Equation 2.26 that the fermion Yukawa couplings are proportional to the mass of the fermion, which is very small except in the case of the top quark. This will be important when considering radiative corrections to the Higgs boson mass involving fermion loops. 
After EWSB, the Higgs potential in the unitary gauge becomes $[27,28]$

$$
V(\Phi)=-\frac{\mu^{4}}{4 \lambda}-\mu^{2} h^{2}+\lambda v h^{3}+\frac{\lambda}{4} h^{4}
$$

The first term of Equation 2.27 is a constant and is irrelevant to physics in the scalar sector. The third and fourth terms represent the cubic and quartic self-interactions of the Higgs boson. The second term represents a tree-level mass

$$
M_{h}^{2}=-2 \mu^{2}=2 v^{2} \lambda
$$

The parameter $v$ can be found from the charged current for muon decay, $\mu^{-} \rightarrow$ $W^{-} \nu_{\mu} \rightarrow e^{-} \bar{\nu}_{e} \nu_{\mu}$. Since the momentum carried by the $W$ boson is on the order of the muon mass, it can be neglected compared to $M_{W}$, and this process can be considered an effective interaction of four fermions with coupling $G_{F}$, following the work of Fermi [32]. This yields

$$
\frac{G_{F}}{\sqrt{2}}=\frac{g^{2}}{8 M_{W}^{2}}=\frac{1}{2 v^{2}}
$$

where $G_{F}=(1.16637 \pm 0.00001) \times 10^{-5} \mathrm{GeV}^{-2}$ [1] is the interaction strength for muon decay. This yields

$$
v=\left(\sqrt{2} G_{F}\right)^{-\frac{1}{2}}=246 \mathrm{GeV}
$$

Now, since $v^{2}=-\frac{\mu^{2}}{\lambda}=(246 \mathrm{GeV})^{2}$ and $M_{h}^{2}=2 v^{2} \lambda$, the free parameters $\mu$ and $\lambda$ can be expressed in terms of $M_{h}$. Therefore, Higgs production and decay processes can be computed in terms of the Higgs mass alone. Unfortunately, the tree-level Higgs boson mass given by Equation 2.28 is a free parameter, the value of which was unknown prior to July 2012 as it had not yet been discovered. Experimental results relevant to the search for the Standard Model Higgs boson are discussed in Section 2.5. 


\subsection{The Higgs Boson Mass}

The Higgs boson enters into one-loop radiative corrections in the Standard Model. Therefore, precision electroweak measurements may be used to constrain the Higgs mass. For example, the radiative corrections to $M_{W}$ can be written as

$$
\frac{M_{W}^{2}}{M_{Z}^{2}}=1-\frac{\pi \alpha}{\sqrt{2} G_{F} M_{W}^{2}(1-\delta r)}
$$

where $\delta r$ is a function of $M_{t}^{2}$ and $\ln \left(M_{h}\right)$ [28]. Precision electroweak measurements as of July 2010 give a $95 \%$ confidence level upper limit on the Higgs boson mass of [1]

$$
114.4 \mathrm{GeV}<M_{h}<149 \mathrm{GeV}
$$

where the lower limit is the LEP-2 direct search limit [33].

Although the Higgs boson mass is a free parameter in the electroweak theory, these results suggest that the Higgs boson is relatively light, on the order of a few hundred $\mathrm{GeV}$. Early 2012 results from the ATLAS experiment at the LHC, using $4.9 \mathrm{fb}^{-1}$ of integrated luminosity at a centre of mass energy of $\sqrt{s}=7 \mathrm{TeV}$, show an excess of events around $126 \mathrm{GeV}$ in three decay channels: $h \rightarrow \gamma \gamma, h \rightarrow Z Z \rightarrow \ell^{+} \ell^{-} \ell^{+} \ell^{-}, h \rightarrow$ $W^{+} W^{-} \rightarrow \ell^{+} \nu_{\ell} \ell^{-} \bar{\nu}_{\ell}$, with statistical significances of $2.8 \sigma, 2.1 \sigma, 1.4 \sigma$ respectively [34]. Combining these three channels results in a statistical significance of $3.5 \sigma$. The CMS experiment at the LHC, including the $h \rightarrow b \bar{b}, h \rightarrow \tau^{+} \tau^{-}$channels as well, reported a similar excess of events around $124 \mathrm{GeV}$ with a statistical significance of $3.1 \sigma$ [35]. While these results are encouraging, a significance of $5 \sigma$ is the criteria needed to claim discovery of any new particles. With $5.9 \mathrm{fb}^{-1}$ of integrated luminosity, including data at a centre of mass energy of $\sqrt{s}=8 \mathrm{TeV}$, the ATLAS Collaboration reported an excess of events near $126.5 \mathrm{GeV}$ with a significance of $5 \sigma$ in July 2012 [3]. With 5.3 $\mathrm{fb}^{-1}$ of integrated luminosity, including data at a centre of mass energy of $\sqrt{s}=8 \mathrm{TeV}$, 
the CMS Collaboration also reported an excess of events at $125.3 \pm 0.4$ (stat) \pm 0.5 (syst) $\mathrm{GeV}$ with a significance of $5 \sigma$ in July 2012 [4]. Although this is evidence for discovery, further analysis is required in order to determine whether this excess of events is actually due to the Standard Model Higgs boson. Nevertheless, these experimental results are consistent with the scenario of a relatively light Higgs boson.

\subsection{The Hierarchy Problem}

Most theorists believe that the Higgs mechanism of the Standard Model is not a complete theory of electroweak symmetry breaking. For example, the Higgs mechanism does not explain why $v=246 \mathrm{GeV}$ and does not explain why there are three generations of fermions having the masses that they do. However, perhaps the most serious problem with the Standard Model, known as the hierarchy or fine-tuning problem, is that loop corrections to the square of the tree-level (bare) Higgs boson mass contain a quadratic divergence, dependent on physics at some higher energy scale, $\Lambda$. For example, when the one-loop corrections, shown in Figure 2.1, to the square of the Higgs boson mass, $\delta M_{h}^{2}$, are calculated, the result is

$$
M_{h}^{2}=\left(M_{h}^{2}\right)_{b a r e}+\delta M_{h}^{2}
$$

where $\left(M_{h}^{2}\right)_{b a r e}$ is given by Equation 2.28 and the one-loop corrections are given by

$$
\delta M_{h}^{2}=\frac{G_{F} \Lambda^{2}}{4 \sqrt{2} \pi^{2}}\left(6 M_{W}^{2}+3 M_{Z}^{2}+M_{h}^{2}-12 M_{t}^{2}\right)+\ldots
$$

where the additional terms in Equation 2.34 are not divergent in $\Lambda$ [36].

At each order in perturbation theory, counterterms could be added to cancel the divergent terms in Equation 2.34, in which case the Higgs mass would be expressed 

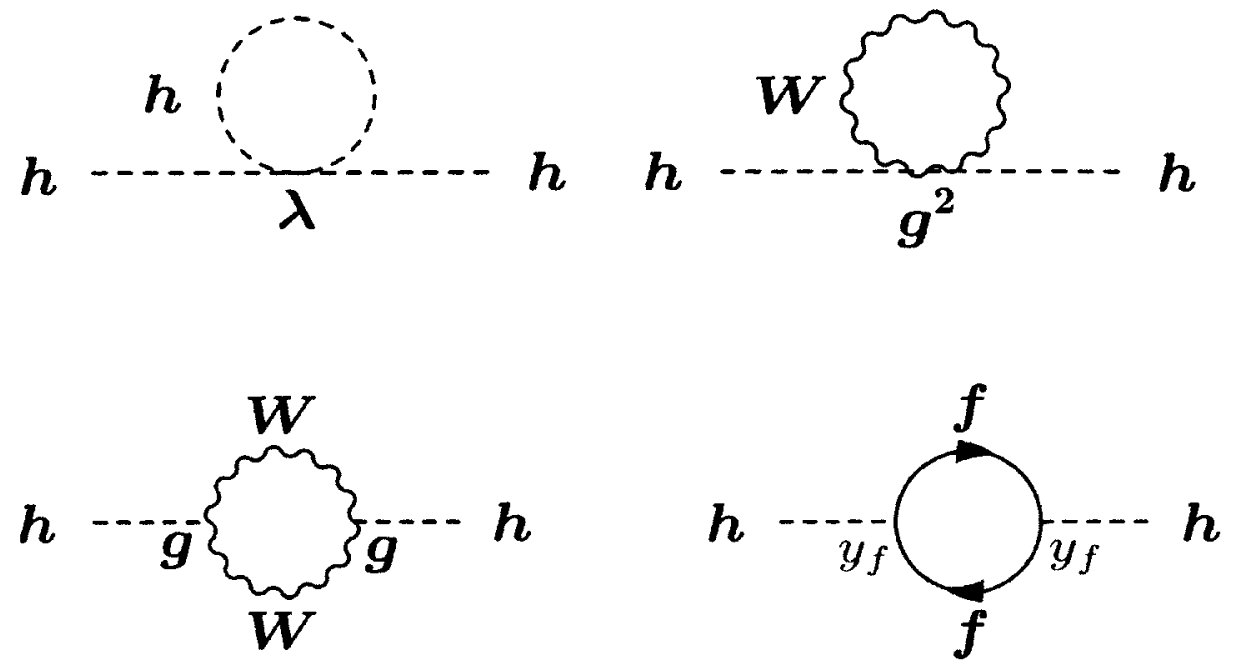

Figure 2.1: Divergent loop contributions to the Higgs boson mass

as

$$
M_{h}^{2}=\left(M_{h}^{2}\right)_{b a r e}+\delta M_{h}^{2}+\text { counterterms }
$$

where the counterterms must be adjusted in order to cancel the quadratically divergent contributions to $\delta M_{h}^{2}[27,28]$. If the Standard Model is valid up to the Planck scale, $\Lambda=10^{19} \mathrm{GeV}$, the counterterms must be fine-tuned to many decimal places in order to cancel the divergent contributions to $\delta M_{h}^{2}$. Most theorists regard this solution to the hierarchy problem as unattractive due to the absence of a natural mechanism in the Standard Model that necessitates such a large fine-tuning.

Consequently, several types of models of physics beyond the Standard Model, such as Supersymmetry, Dynamical Symmetry Breaking, and Extra Dimensions, have offered possible solutions to the hierarchy problem [10-12]. However, much of the parameter space of these models, particularly Supersymmetry, is being ruled out as the LHC collects more data [37]. Another relatively recent class of models, known as Little Higgs models, offers an alternative solution to the hierarchy problem, as 
described in Section 2.7 .

\subsection{Little Higgs Models}

Little Higgs models [13-17,38] are a relatively new class of models for physics beyond the Standard Model that attempt to solve the hierarchy problem. There are several variations of Little Higgs models (for recent reviews, consult [38-41]), such as the Simplest Little Higgs model [42], the Littlest Higgs model [17], and Little Higgs models with $T$-parity [43-45]. Little Higgs models resolve the hierarchy problem by introducing new particles at a scale of $\sim 1 \mathrm{TeV}$ in addition to the particles of the Standard Model. The couplings of these particles to the Higgs boson are such that the quadratic divergences induced by loops of Standard Model particles are cancelled by the quadratic divergences induced by loops of the $\mathrm{TeV}$ scale particles at one-loop level. This cancellation occurs between particles with the same statistics; divergences due to the Standard Model gauge bosons are cancelled by the contributions of new heavy gauge bosons, divergences from the top quark Yukawa coupling are cancelled by the contributions of new heavy fermions, and divergences due to the Higgs boson loops are cancelled by the contribution of new heavy scalar particles.

Although these new $\mathrm{TeV}$ scale particles are required to ensure that the Higgs boson is relatively light, on the order of the weak scale, constraints from precision electroweak measurements reveal no evidence for new physics below approximately 10 $\mathrm{TeV}$ [46]. Little Higgs models are named as such because they also solve this so-called "little" hierarchy problem by raising the cutoff scale to $\Lambda_{S} \sim 10 \mathrm{TeV}$. The Standard Model Higgs boson remains light and free from one-loop quadratic divergences without introducing fine-tuning up to $\Lambda_{S}$, while at energies greater than $\Lambda_{S}$, the model becomes strongly coupled and new physics emerges [17]. Any new physics above $\Lambda_{S}$ 
should not affect precision electroweak measurements and it is assumed that the contributions to flavour physics are not dangerous [46]. Furthermore, the new physics above $\Lambda_{S}$ does not affect the LHC phenomenology presented in this thesis.

The key ingredient of Little Higgs models that allows them to solve the hierarchy problem is known as Collective Symmetry Breaking. In the Higgs sector, for example, the Higgs quartic coupling is introduced through two operators, both of which individually preserve enough symmetries to forbid quadratic divergences in $M_{h}^{2}$, but collectively generate the desired Higgs potential. This generally requires the existence of additional scalars other than the Standard Model Higgs boson [47].

Concretely, suppose the Higgs potential is of the form

$$
V \sim \lambda_{1} f^{2}\left|\phi+\frac{h^{2}}{f}+\ldots\right|^{2}+\lambda_{2} f^{2}\left|\phi-\frac{h^{2}}{f}+\ldots\right|^{2}
$$

where $h$ denotes the Higgs field and $\phi$ is an additional scalar field [47]. Consider the shift symmetries given by [47]

$$
\begin{array}{ll}
h \rightarrow h+\epsilon+\ldots, & \phi \rightarrow \phi-\frac{h \epsilon+\epsilon h}{f}+\ldots, \\
h \rightarrow h+\epsilon+\ldots, & \phi \rightarrow \phi+\frac{h \epsilon+\epsilon h}{f}+\ldots .
\end{array}
$$

The two operators from the $\lambda_{1}$ and $\lambda_{2}$ terms of Equation 2.36 are each invariant under the the shift symmetries of Equations 2.37 and 2.38, respectively. Taken alone, neither term in Equation 2.36 gives a physical Higgs quartic, since it can simply be removed by a field redefinition:

$$
\phi \rightarrow \phi \mp \frac{h^{2}}{f}+\ldots
$$

However, collectively, both operators of Equation 2.36 yield a Higgs quartic after the 
scalar $\phi$ is integrated out. This can be seen by minimizing the potential with respect to $\phi$ and substituting the resulting solution for $\phi$ into Equation 2.36, as follows:

$$
\begin{aligned}
\left.\frac{\partial V}{\partial \phi}\right|_{\phi=\phi_{0}}=0 & \Longrightarrow 2 \lambda_{1} f^{2}\left(\phi+\frac{h^{2}}{f}+\ldots\right)+\left.2 \lambda_{2} f^{2}\left(\phi-\frac{h^{2}}{f}+\ldots\right)\right|_{\phi=\phi_{0}}=0 \\
& \Longrightarrow \phi_{0}=\frac{\lambda_{2}-\lambda_{1}}{\lambda_{1}+\lambda_{2}} \frac{h^{2}}{f}+\ldots
\end{aligned}
$$

Therefore,

$$
V\left(\phi=\phi_{0}\right) \sim \lambda_{1}\left(\frac{2 \lambda_{2}}{\lambda_{1}+\lambda_{2}}\right)^{2} h^{4}+\lambda_{2}\left(\frac{2 \lambda_{1}}{\lambda_{1}+\lambda_{2}}\right)^{2} h^{4}=4 \frac{\lambda_{1} \lambda_{2}}{\lambda_{1}+\lambda_{2}} h^{4}
$$

One notices that the Higgs quartic generated in Equation 2.41 vanishes if either $\lambda_{1}=0$ or $\lambda_{2}=0$ (if both $\lambda_{1}$ and $\lambda_{2}$ are zero, the potential of Equation 2.36 vanishes altogether and a quartic cannot be generated). This is the essence of collective symmetry breaking and reaffirms that both operators of Equation 2.36 are indeed required to generate a Higgs quartic.

As a concrete example of a Little Higgs model, consider the Littlest Higgs model [17] (an alternative Little Higgs model, dubbed the Bestest Little Higgs, will be studied in Chapters 4 and 5). Electroweak Symmetry Breaking in the Littlest Higgs model is more complicated than the Higgs mechanism of the Standard Model, and proceeds as follows $[17,39]$. The Littlest Higgs model begins with an $S U(5)$ global symmetry with a locally gauged subgroup $[S U(2) \times U(1)]^{2}$. At a scale $\Lambda_{S} \sim 4 \pi f \sim$ $10 \mathrm{TeV}$, the $S U(5)$ global symmetry is broken down to its subgroup $S O(5)$ via a vev of order $f \sim 1 \mathrm{TeV}$. At the same time, the gauge symmetry $[S U(2) \times U(1)]^{2}$ is broken down to its diagonal subgroup $S U(2)_{L} \times U(1)_{Y}$, identified as the electroweak gauge group of the Standard Model. As the $S U(N)$ groups have $N^{2}-1$ symmetry generators and the $S O(N)$ groups have $N(N-1) / 2$ generators, The $S U(5) \rightarrow S O(5)$ symmetry breaking leaves $\left[5^{2}-1\right]-[5(5-1) / 2]=14$ Pseudo Nambu-Goldstone 
Bosons (PNGBs), corresponding to the broken generators. Under $S U(2)_{L} \times U(1)_{Y}$, these transform as

$$
\mathbf{1}_{0} \oplus \mathbf{3}_{0} \oplus \mathbf{2}_{ \pm \frac{1}{2}} \oplus \mathbf{3}_{ \pm 1}
$$

The real singlet, $\mathbf{1}_{0}$, and the real triplet, $\mathbf{3}_{0}$, become the longitudinal components of the new heavy gauge bosons, $W^{ \pm}$and $Z^{\prime}$, which acquire masses on the order of the scale $f$. The complex doublet, $\mathbf{2}_{ \pm \frac{1}{2}}$, is identified as the Standard Model Higgs boson, and $\mathbf{3}_{ \pm 1}$ is an additional complex triplet of heavy Higgs bosons. At this stage of the symmetry breaking, these Higgs fields remain massless. The Littlest Higgs model also predicts the existence of a charge $2 / 3$ heavy top partner, $T$, with a mass of order $f$; this is required to cancel the quadratically divergent contribution to the square of the Higgs boson mass due to the Standard Model top quark loop, which has by far the largest Yukawa coupling of all the Standard Model fermions. No partners for the remaining light fermions are introduced because their Yukawa couplings are small enough that the one-loop quadratically divergent contributions they induce are negligible up to a cutoff $\Lambda_{S} \sim 10 \mathrm{TeV}$ [17]. As the heavy fermion and heavy gauge sectors of this model have been studied extensively in the literature [39-41, 48-50], the focus of the remainder of this section and of Chapter 3 of this thesis is the scalar sector of the Littlest Higgs model.

The PNGBs can be parameterized by a non-linear sigma model field

$$
\Sigma=e^{i \Pi / f} \Sigma_{0} e^{i \Pi \Pi^{T} / f}
$$

where $\Sigma_{0}=\langle\Sigma\rangle \sim f$ is the vev associated with the symmetry breaking $S U(5) \rightarrow$ 
$S O(5)$, and the $5 \times 5$ PNGB matrix, $\Pi$, is given by

$$
\Pi=\left(\begin{array}{ccc}
0_{2 \times 2} & h^{\dagger} / \sqrt{2} & \phi^{\dagger} \\
h / \sqrt{2} & 0 & h^{*} / \sqrt{2} \\
\phi & h^{T} / \sqrt{2} & 0_{2 \times 2}
\end{array}\right) .
$$

The scalar field content consists of a doublet, $h$, and a triplet, $\phi$, under the unbroken $S U(2)_{L} \times U(1)_{Y}$ Standard Model gauge group:

$$
h=\left(h^{+}, h^{0}\right), \quad \phi=\left(\begin{array}{cc}
\phi^{++} & \frac{\phi^{+}}{\sqrt{2}} \\
\frac{\phi^{+}}{\sqrt{2}} & \phi^{0}
\end{array}\right) .
$$

At this stage in the symmetry breaking, the scalar doublet and triplet remain massless. The masses of these particles are generated through a second step in the symmetry breaking, as described below.

In the Littlest Higgs model, the symmetries of Equations 2.37-2.38 prevent the appearance of a Higgs potential at tree-level. Instead, the gauge and Yukawa couplings that break the global $S O(5)$ symmetry will radiatively induce a Higgs potential at one-loop and higher orders. This potential, named the Coleman-Weinberg potential [51], is given by [39]

$$
V=\lambda_{\phi^{2}} f^{2} \operatorname{Tr}\left(\phi^{\dagger} \phi\right)+i \lambda_{h \phi h} f\left(h \phi^{\dagger} h^{T}-h^{*} \phi h^{\dagger}\right)-\mu^{2} h h^{\dagger}+\lambda_{h^{4}}\left(h h^{\dagger}\right)^{2}
$$

where terms involving $\phi^{4}$ and $h^{2} \phi^{2}$ have been neglected since their contribution is small.

For $\mu^{2}>0$, this scalar potential triggers electroweak symmetry breaking as the 
neutral components of the doublet and triplet fields acquire vacuum expectation values, given by [39]:

$$
\begin{aligned}
\left\langle h^{0}\right\rangle & =\frac{v}{\sqrt{2}} \\
\left\langle i \phi^{0}\right\rangle & =v^{\prime}
\end{aligned}
$$

with

$$
v^{2}=\frac{\mu^{2}}{\lambda_{h^{4}}-\lambda_{h \phi h}^{2} / \lambda_{\phi^{2}}}, \quad v^{\prime}=\frac{\lambda_{h \phi h}}{2 \lambda_{\phi^{2}}} \frac{v^{2}}{f} .
$$

Note that because of the factor of $i$ in the second term of the potential of Equation 2.46, the physical triplet mass eigenstates and the triplet vev of Equation 2.48 are defined to include this factor of $i$ [39]. Minimizing the potential also leads to the relation

$$
\frac{\lambda_{h \phi h}}{\lambda_{h^{4}}}=\frac{4 \lambda_{h \phi h}}{\lambda_{\phi^{2}}}=\frac{8 v^{\prime}}{v} \frac{f}{v} .
$$

The Higgs doublet and triplet states acquire masses, which to lowest order, are given by $[39]$

$$
\begin{aligned}
& M_{h}^{2} \simeq 2\left(\lambda_{h^{4}}-\lambda_{h \phi h}^{2} / \lambda_{\phi^{2}}\right) v^{2}=2 \mu^{2} \\
& M_{\Phi}^{2} \simeq \lambda_{\phi^{2}} f^{2} .
\end{aligned}
$$

It is interesting to note that to lowest order, the triplet states are degenerate in mass. Using Equations 2.49 and 2.50 to solve for the couplings allows one to express the triplet mass in terms of the physical parameters $f, M_{h}^{2}, v$ and $v^{\prime}$ as

$$
M_{\Phi}^{2}=\frac{2 M_{h}^{2} f^{2}}{v^{2}} \frac{1}{\left[1-\left(4 v^{\prime} f / v^{2}\right)^{2}\right]} .
$$

The states of the heavy Higgs triplet can be produced at the Large Hadron Collider 
via vector boson fusion. Due to its distinctive final state signature, $\Phi^{ \pm \pm}$production via $W^{ \pm} W^{ \pm}$scattering is an interesting process to study at the LHC. Perhaps more interesting is the singly-charged state, $\Phi^{ \pm}$, which can be produced via $W^{ \pm} Z$ scattering. This is an interesting channel with a charged Higgs, as the $W^{+} Z \Phi^{-}$coupling does not exist at tree-level in Two Higgs Doublet Models such as Supersymmetry [52]. Therefore, if the process $W^{ \pm} Z \rightarrow \Phi^{ \pm} \rightarrow W^{ \pm} Z$ is experimentally observed, it would provide evidence for the existence of a Higgs triplet, so it is worth studying the potential for discovering Higgs triplets at the LHC. The vector boson scattering signals of the Littlest Higgs model [17], as well as those of two other Higgs triplet models, the Georgi-Machacek model [18] and the Left-Right Symmetric model [19,20], are discussed in Chapter 3. 


\section{Chapter 3}

\section{Exploring Higgs Triplet Models Via}

\section{Vector Boson Scattering at the Large}

\section{Hadron Collider}

\subsection{Introduction}

It has been pointed out that doubly-charged members of Higgs triplets $\left(\Phi^{ \pm \pm}\right)$might serve as a good signal for Little Higgs models if they are kinematically accessible at future colliders [39]. Han et al. [39] studied the effects of resonant contributions from $\Phi^{++} \rightarrow W^{+} W^{+}$in an idealized calculation of longitudinal $W^{+} W^{+}$scattering, $W_{L}^{+} W_{L}^{+} \rightarrow W_{L}^{+} W_{L}^{+}$, in the Littlest Higgs model. A more detailed analysis by Azuelos et al. [49] found that a $\Phi^{++}$could be observed up to $2 \mathrm{TeV}$ with the right range of parameters. However, the Littlest Higgs model also predicts neutral $\left(\Phi^{0}\right)$ and singlycharged $\left(\Phi^{ \pm}\right)$members of the Higgs triplet with $W^{+} W^{-} \Phi^{0}, Z Z \Phi^{0}$, and $W^{+} Z \Phi^{-}$ interactions. If heavy scalars were observed, it would be necessary to map out their properties to construct the underlying Lagrangian. For example, the $W^{+} Z \Phi^{-}$vertex is unique to Higgs triplet representations and does not exist at tree-level in Two Higgs Doublet Models (2HDM), so the observation of this interaction would provide 
evidence for Higgs triplets [52].

The observation of a Higgs triplet state would not, however, uniquely identify the model of origin [52]. In addition to the Littlest Higgs model, scalar triplets are also present in the Georgi-Machacek model [18] and Left-Right Symmetric models $[19,20]$. The so-called 3-3-1 models $[53,54]$ are another class of models that include Higgs triplets [55]. However, because 3-3-1 models are similar to variations of Little Higgs models [42], they will not be considered separately. To distinguish between models, one therefore needs to study the properties of newly discovered scalar resonances. In this chapter, vector boson scattering is examined to determine whether it can be used to distinguish between models that include Higgs triplet bosons. Vector boson pair production was also considered as a signature of Higgsless models [56-58] in the $W Z$ and $W^{+} W^{-}$channels [59-61] but the study of Higgsless models is not included in this thesis.

Higgs bosons can in principle couple to fermions and, in fact, a same-sign dilepton final state would almost certainly be a cleaner signal for a doubly-charged Higgs boson than vector boson final states. Although this lepton number violating decay, $\Phi^{ \pm \pm} \rightarrow \ell^{ \pm} \ell^{ \pm}$, does not occur in the version of the Littlest Higgs model considered in this thesis, it can occur in certain variations of the Littlest Higgs model [62], as well as in the Georgi-Machacek [63] and Left-Right Symmetric models [64-66]. It is therefore worth commenting on the relative strengths of the dilepton and diboson channels. Neutrino masses are an important constraint on the allowed parameter range, which in turn leads to different decay scenarios for the Higgs triplet states [62]. In one case, the small neutrino mass can be driven by a tiny triplet vacuum expectation value (vev), but with neutrino Yukawa couplings $\mathcal{O}(1)$, while in the opposite scenario it is the Yukawa couplings that are tiny to accomodate the observed neutrino masses. It is the latter case which can lead to a triplet vev that is large enough to give rise to 
the signal being studied here.

In this chapter, a study [67] of heavy scalar triplet production via vector boson scattering in the $W^{ \pm} W^{ \pm}, W^{ \pm} Z, W^{+} W^{-}$, and $Z Z$ channels is presented. The purely leptonic decays of the vector bosons are examined, as these decay channels have the advantage of lower Standard Model QCD backgrounds. The final states considered are therefore $W^{ \pm} W^{ \pm} \rightarrow \ell^{ \pm} \nu \ell^{ \pm} \nu, W^{ \pm} Z \rightarrow \ell^{ \pm} \nu \ell^{+} \ell^{-}, W^{+} W^{-} \rightarrow \ell^{+} \nu \ell^{-} \bar{\nu}$ and $Z Z \rightarrow$ $\ell^{+} \ell^{-} \ell^{+} \ell^{-}$. These processes have been extensively studied in the context of strongly interacting weak sector models [68-71] and previous studies of doubly-charged Higgs boson production at the LHC in the Littlest Higgs and Left-Right Symmetric models were given by Azuelos et al. $[49,66]$.

In Section 3.2, the models under study are outlined, focusing on properties of the scalar sector most relevant to the vector boson scattering processes. This is followed in Section 3.3 by a description of calculational details. Backgrounds and the kinematic cuts that were used to reduce them are described in Section 3.4. The results of this study are given in Section 3.5 followed by a brief summary of conclusions in Section 3.6 .

\subsection{Models}

A brief overview of the Littlest Higgs, Georgi-Machacek, and Left-Right Symmetric models is presented in this section. It is not intended to be an extensive review but only gives details used in the analysis of vector boson scattering. The Feynman rules needed for the calculations are summarized in Table 3.1. 


\subsubsection{The Littlest Higgs Model}

As described in Section 2.7, the scalar field content of the Littlest Higgs model consists of a doublet, $h$, and a triplet, $\phi$, under the unbroken $S U(2)_{L} \times U(1)_{Y}$ Standard Model gauge group:

$$
h=\left(\begin{array}{c}
h^{+} \\
h^{0}
\end{array}\right), \quad \phi=\left(\begin{array}{cc}
\phi^{++} & \phi^{+} / \sqrt{2} \\
\phi^{+} / \sqrt{2} & \phi^{0}
\end{array}\right) .
$$

Electroweak symmetry breaking results in a vev for the neutral components of both the doublet and the triplet fields: $\left\langle h^{0}\right\rangle=v / \sqrt{2}$ and $\left\langle i \phi^{0}\right\rangle=v^{\prime}$. The doublet and triplet states acquire masses, the latter of which is given to leading order by Equation 2.52 [39]. Demanding that $M_{\Phi}^{2}>0$, gives a relation between the doublet and triplet vevs $[17,39]$ :

$$
\frac{v^{\prime}}{v}<\frac{v}{4 f} .
$$

For $f=2 \mathrm{TeV}$ and $v=246 \mathrm{GeV}$, this gives an upper bound

$$
v^{\prime}<8 \mathrm{GeV} \text {. }
$$

The value of $v^{\prime}$ has been further constrained by electroweak data to $1 \mathrm{GeV} \lesssim v^{\prime} \lesssim 4$ $\mathrm{GeV}$ for $f=2 \mathrm{TeV}$ [72]. This small upper bound on $v^{\prime}$ implies a certain amount of fine-tuning in order to preserve a custodial symmetry, which requires that the $\rho$ parameter in this model is $\rho=1$. However, such a fine-tuning does not occur in the Georgi-Machacek model [18], which explicitly preserves a custodial symmetry and guarantees that $\rho=1$ regardless of the value of $v^{\prime}$. This will be seen in Section 3.2.2. 


\subsubsection{The Georgi-Machacek Model}

The scalar fields of the Georgi-Machacek model [18] take the form

$$
\phi\left(\frac{1}{2}, \frac{1}{2}\right)=\left(\begin{array}{c}
\phi^{+} \\
\phi^{0}
\end{array}\right), \quad \chi(1,1)=\left(\begin{array}{ccc}
\chi^{0} & \xi^{+} & \chi^{++} \\
\chi^{-} & \xi^{0} & \chi^{+} \\
\chi^{--} & \xi^{-} & \chi^{0^{*}}
\end{array}\right)
$$

where the $S U(2)_{L}$ and $S U(2)_{R}$ quantum numbers of the fields are indicated in parentheses. Therefore, $\phi$ and $\chi$ given by Equation 3.4 transform as a doublet and a triplet, respectively, under $S U(2)_{L} \times S U(2)_{R}$. Their potential is determined by writing the most general potential that is symmetric under the full global $S U(2)_{L} \times S U(2)_{R}$ symmetry [73]. $\phi$ is a complex doublet, $\xi$ is a real triplet and $\chi$ is a complex triplet. Electroweak symmetry breaking occurs when the neutral components of these fields develop a non-zero vev, given by $\left\langle\phi^{0}\right\rangle=a / \sqrt{2}$ and $\left\langle\chi^{0}\right\rangle=\left\langle\xi^{0}\right\rangle=v^{\prime}$. It is convenient to introduce the notation

$$
\begin{aligned}
v^{2} & \equiv a^{2}+8 v^{\prime 2} \\
c_{H}^{2} & \equiv \frac{a^{2}}{v^{2}}, \quad s_{H}^{2} \equiv \frac{8 v^{\prime 2}}{v^{2}}
\end{aligned}
$$

as, in this model, the $W^{ \pm}$and $Z$ bosons masses are given by

$$
M_{W}^{2}=M_{Z}^{2} \cos ^{2} \theta_{W}=\frac{1}{4} g^{2}\left(a^{2}+8 v^{2}\right)=\frac{1}{4} g^{2} v^{2}
$$

The relations given by Equations 3.5, 3.6 and 3.7 were substituted into the Feynman rules given by [63] so that the Feynman rules in Table 3.1 use a common notation. 
The physical scalars in this model can be classified according to their transformation properties under the custodial $S U(2)$ symmetry; one finds a five-plet $\left(H_{5}^{ \pm \pm}, H_{5}^{ \pm}\right.$, $H_{5}^{0}$ ), a three-plet $\left(H_{3}^{ \pm}, H_{3}^{0}\right)$, and two singlets, $H_{1}^{0}$ (which is identified as the Standard Model Higgs boson) and $H_{1}^{0 \prime}$. Production of the $H_{5}$ triplet is the focus of this analysis as these particles couple to the $W^{ \pm}$and $Z$ bosons, whereas the $H_{3}$ triplet does not.

From Equation 3.7, it is evident that $v=\sqrt{a^{2}+8 v^{\prime 2}} \approx 246 \mathrm{GeV}$, but the ratio $v^{\prime} / a$ is not fixed. The strongest experimental bound on this ratio comes from the $Z b \bar{b}$ coupling measurements, which give, at $95 \%$ confidence level, $\tan \theta_{H} \equiv s_{H} / c_{H} \lesssim 0.5,1$ and 1.7 for $M_{H_{3}}=0.1,0.5$ and $1 \mathrm{TeV}$, respectively [74]. A value of $\tan \theta_{H}=0.5$ corresponds to a triplet vev of $v^{\prime}=39 \mathrm{GeV}$. It is evident that the triplet vev in this model is allowed to be larger than that of the Littlest Higgs model without violating precision electroweak constraints. This is because the custodial symmetry of the Georgi-Machacek model eliminates contributions to the electroweak Peskin-Takeuchi $T$-parameter [75], or equivalently, guarantees that the $\rho$ parameter is equal to $\rho=1$. 


\subsubsection{The Left-Right Symmetric Model}

The scalar sector of the $S U(2)_{L} \times S U(2)_{R} \times U(1)_{B-L}$ Left-Right Symmetric model $[19,20]$ is given by $[65,76]$

$$
\begin{aligned}
\phi\left(\frac{1}{2}, \frac{1}{2}, 0\right) & =\left(\begin{array}{cc}
\phi_{1}^{0} & \phi_{1}^{+} \\
\phi_{2}^{-} & \phi_{2}^{0}
\end{array}\right), \\
\Delta_{L}(1,0,2) & =\left(\begin{array}{cc}
\Delta_{L}^{+} / \sqrt{2} & \Delta_{L}^{++} \\
\Delta_{L}^{0} & -\Delta_{L}^{+} / \sqrt{2}
\end{array}\right), \\
\Delta_{R}(0,1,2) & =\left(\begin{array}{cc}
\Delta_{R}^{+} / \sqrt{2} & \Delta_{R}^{++} \\
\Delta_{R}^{0} & -\Delta_{R}^{+} / \sqrt{2}
\end{array}\right),
\end{aligned}
$$

where the $S U(2)_{L}, S U(2)_{R}$ and $B-L$ quantum numbers of the fields are indicated in parentheses. Therefore, $\phi$ transforms as a doublet under $S U(2)_{L}$ and $S U(2)_{R}, \Delta_{L}$ is an $S U(2)_{L}$ triplet and $\Delta_{R}$ is an $S U(2)_{R}$ triplet. Left-right symmetry requires that the Lagrangian of the theory is invariant under $\Delta_{L} \leftrightarrow \Delta_{R}$ and $\phi \leftrightarrow \phi^{\dagger}$. The vacuum expectation values of the doublet and triplet are given by

$$
\begin{aligned}
\langle\phi\rangle & =\frac{1}{\sqrt{2}}\left(\begin{array}{cc}
\kappa_{1} & 0 \\
0 & \kappa_{2}
\end{array}\right), \\
\left\langle\Delta_{L, R}\right\rangle & =\frac{1}{\sqrt{2}}\left(\begin{array}{cc}
0 & 0 \\
v_{L, R} & 0
\end{array}\right) .
\end{aligned}
$$


The triplet vevs $\left(v_{L}, v_{R}\right)$ break $S U(2)_{L} \times S U(2)_{R} \times U(1)_{B-L} \rightarrow S U(2)_{L} \times U(1)_{Y}$, while the doublet, $\phi$, breaks $S U(2)_{L} \times U(1)_{Y} \rightarrow U(1)_{E M}$.

The focus of this study is the production of the left triplet, $\Delta_{L}$, as it couples to the Standard Model $W^{ \pm}$and $Z$ bosons. The left triplet vev, $v_{L}$, is constrained by the $\rho$ parameter, given by $[64,65]$ :

$$
\rho \equiv \frac{M_{W}^{2}}{\cos ^{2} \theta_{W} M_{Z}^{2}} \simeq \frac{1+2 v_{L}^{2} / v^{2}}{1+4 v_{L}^{2} / v^{2}}
$$

The experimental result $\rho=1.0004_{-0.0007}^{+0.0027}$ at $2 \sigma[77]$ implies that $v_{L} \lesssim 3 \mathrm{GeV}$, a small value compared with $v \equiv \sqrt{\kappa_{1}^{2}+\kappa_{2}^{2}} \approx 246 \mathrm{GeV}$. Note that due to the factor of $1 / \sqrt{2}$ in Equation 3.12, the triplet vev in this model is defined as $v^{\prime} \equiv v_{L} / \sqrt{2}$ to be consistent with the conventions of the other models under study. Using this notation, the upper limit on the triplet vev becomes $v^{\prime} \lesssim 2 \mathrm{GeV}$.

\subsection{Calculations}

The production mechanism for scalar triplet bosons via vector boson scattering at the LHC is illustrated in Figure 3.1. The incoming quarks from the colliding protons radiate vector bosons $\left(V=W^{ \pm}, Z\right)$, which interact to produce the Higgs triplet. The Higgs triplet then decays to vector bosons, accompanied by two spectator quark jets that emerge in the forward region of the detector. The forward jets are the hallmark of the vector boson fusion process and are an important tool to reduce backgrounds. The production of all members of the scalar triplet is studied: $\Phi^{ \pm \pm}$via $W^{ \pm} W^{ \pm}$ scattering, $\Phi^{ \pm}$via $W^{ \pm} Z$ scattering, and $\Phi^{0}$ via $W^{+} W^{-}$and $Z Z$ scattering.

To calculate the $p p \rightarrow \Phi+j j \rightarrow V V+j j$ cross sections and kinematic distributions, the MadGraph software package, version 4.2.6 [78] was used. MadGraph generates tree-level matrix elements and then generates events using Monte Carlo 
Table 3.1: Feynman rules for the interactions of scalar and vector bosons in the Littlest Higgs [39], Georgi-Machacek [63] and Left-Right Symmetric models [65].

\begin{tabular}{|c|c|}
\hline \multicolumn{2}{|r|}{ Littlest Higgs Model } \\
\hline$W_{\mu}^{+} W_{\nu}^{-} h$ & $\frac{i}{2} g^{2} v\left(1-\frac{v^{2}}{3 f^{2}}-\frac{1}{2}\left(c^{2}-s^{2}\right)^{2} \frac{v^{2}}{f^{2}}-\frac{1}{2} s_{0}^{2}-2 \sqrt{2} s_{0} \frac{v^{\prime}}{v}\right) g_{\mu \nu}$ \\
\hline$Z_{\mu} Z_{\nu} h$ & $\begin{array}{l}\frac{i}{2} \frac{g^{2}}{c_{W}^{2}} v\left(1-\frac{v^{2}}{3 f^{2}}-\frac{1}{2} s_{0}^{2}+4 \sqrt{2} s_{0} \frac{v^{\prime}}{v}\right. \\
\left.-\frac{1}{2}\left(\left(c^{2}-s^{2}\right)^{2}+5\left(c^{\prime 2}-s^{2}\right)^{2}\right) \frac{v^{2}}{f^{2}}\right) g_{\mu \nu}\end{array}$ \\
\hline$W_{\mu}^{+} W_{\nu}^{-} \Phi^{0}$ & $-\frac{i}{2} g^{2}\left(s_{0} v-2 \sqrt{2} v^{\prime}\right) g_{\mu \nu} \simeq 0$ \\
\hline$Z_{\mu} Z_{\nu} \Phi^{0}$ & $-\frac{i}{2} \frac{g^{2}}{c_{W}^{2}}\left(s_{0} v-4 \sqrt{2} v^{\prime}\right) g_{\mu \nu} \simeq \sqrt{2} i \frac{g^{2}}{c_{W}^{2}} v^{\prime} g_{\mu \nu}$ \\
\hline$W_{\mu}^{+} Z_{\nu} \Phi^{-}$ & $-i \frac{g^{2}}{c_{W}} v^{\prime} g_{\mu \nu}$ \\
\hline$W_{\mu}^{+} h \Phi^{-}$ & $-i \frac{g}{2}\left(\sqrt{2} s_{0}-s_{+}\right)\left(p_{1}-p_{2}\right)_{\mu} \simeq-i g \frac{v^{\prime}}{v}\left(p_{1}-p_{2}\right)_{\mu}$ \\
\hline$W_{\mu}^{+} W_{\nu}^{+} \Phi^{--}$ & $2 i g^{2} v^{\prime} g_{\mu \nu}$ \\
\hline \multicolumn{2}{|r|}{ Georgi-Machacek Model } \\
\hline$W_{\mu}^{+} W_{\nu}^{-} H_{1}^{0}$ & $\frac{i}{2} g^{2} v\left(1-8 \frac{v^{\prime 2}}{v^{2}}\right) g_{\mu \nu}$ \\
\hline$Z_{\mu} Z_{\nu} H_{1}^{0}$ & $\frac{i}{2} \frac{g^{2}}{c_{W}^{2}} v\left(1-8 \frac{v^{\prime 2}}{v^{2}}\right) g_{\mu \nu}$ \\
\hline$W_{\mu}^{+} W_{\nu}^{-} H_{5}^{0}$ & $\sqrt{\frac{2}{3}} i g^{2} v^{\prime} g_{\mu \nu}$ \\
\hline$Z_{\mu} Z_{\nu} H_{5}^{0}$ & $-2 \sqrt{\frac{2}{3}} i \frac{g^{2}}{c_{W}^{2}} v^{\prime} g_{\mu \nu}$ \\
\hline$W_{\mu}^{+} Z_{\nu} H_{5}^{-}$ & $-\sqrt{2} i \frac{g^{2}}{c_{W}} v^{\prime} g_{\mu \nu}$ \\
\hline$W_{\mu}^{+} H_{1}^{0} H_{5}^{-}$ & 0 \\
\hline$W_{\mu}^{+} W_{\nu}^{+} H_{5}^{--}$ & $2 i g^{2} v^{\prime} g_{\mu \nu}$ \\
\hline$W_{\mu}^{+} W_{\nu}^{-} H_{1}^{0 \prime}$ & $\frac{4}{\sqrt{3}} i g^{2} v^{\prime} g_{\mu \nu}$ \\
\hline$Z_{\mu} Z_{\nu} H_{1}^{0 \prime}$ & $\frac{4}{\sqrt{3}} i \frac{g^{2}}{c_{W}^{2}} v^{\prime} g_{\mu \nu}$ \\
\hline \multicolumn{2}{|r|}{ Left-Right Symmetric Model } \\
\hline$W_{\mu}^{+} W_{\nu}^{-} h$ & $\frac{i}{2} g^{2} v g_{\mu \nu}$ \\
\hline$Z_{\mu} Z_{\nu} h$ & $\frac{i}{2} \frac{g^{2}}{c_{W}^{2}} v g_{\mu \nu}$ \\
\hline$W_{\mu}^{+} W_{\nu}^{-} \Delta_{L}^{0}$ & $\sqrt{2} i g^{2} v^{\prime} g_{\mu \nu}$ \\
\hline$Z_{\mu} Z_{\nu} \Delta_{L}^{0}$ & $2 \sqrt{2} i \frac{g^{2}}{c_{W}^{2}} v^{\prime} g_{\mu \nu}$ \\
\hline$W_{\mu}^{+} Z_{\nu} \Delta_{L}^{-}$ & $-i \frac{g^{2}}{c_{W}} v^{\prime} g_{\mu \nu}$ \\
\hline$W_{\mu}^{+} h \Delta_{L}^{-}$ & 0 \\
\hline$W_{\mu}^{+} W_{\nu}^{+} \Delta_{L}^{-}$ & $-2 i g^{2} v^{\prime} g_{\mu \nu}$ \\
\hline
\end{tabular}




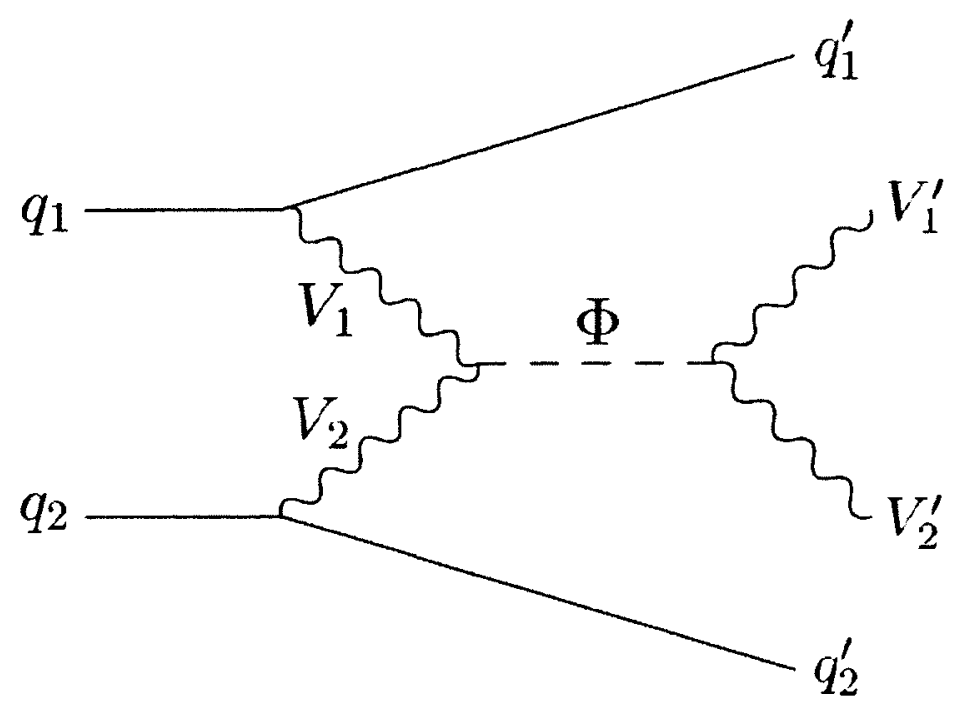

Figure 3.1: Higgs triplet production via vector boson scattering at the LHC. The incoming quarks from the colliding protons radiate vector bosons, which interact to produce a Higgs triplet as an s-channel resonance. Two vector bosons emerge in the final state, along with two forward spectator quark jets.

phase space integration. The parton-level generated events were then analyzed, including implementing kinematic cuts in order to determine the discovery potential in each channel. Although including the effects of parton showering, hadronization and a detector simulation would introduce smearing that degrades the energy resolution, the widths of the scalar triplet bosons are already fairly broad (see Table 3.3), so this is not expected to have a large effect on the results of this study. Although the efficiency for reconstructing the final states under consideration may be reduced by introducing a detector simulation, these effects are beyond the scope of this preliminary analysis of Higgs triplet production, and were not taken into account.

The relevant scalar sector Feynman rules for the Littlest Higgs model [39], the Georgi-Machacek model [63] and the Left-Right Symmetric model [65] were implemented in MadGraph (see Table 3.1). Note that in the Littlest Higgs model, the 
scalar sector mixing angles are given to leading order by $s_{0}=\sqrt{2} s_{+} \simeq 2 \sqrt{2} v^{\prime} / v[39]$. This results in a value of zero for the $W^{+} W^{-} \Phi^{0}$ vertex, so the $W^{+} W^{-}$scattering process was not studied in the Littlest Higgs model. In the $W W h$ and $Z Z h$ vertices of the Littlest Higgs model, the $\mathcal{O}\left(v^{2} / f^{2}\right)$ terms were not included since their contributions are negligible for the purposes of this study, regardless of the values of the mixing angles $\left(s, c, s^{\prime}, c^{\prime}\right)$ in the gauge sector of this model.

It is interesting to note some of the similarities and differences in the Feynman rules of each model. For example, the $W^{+} W^{+} \Phi^{--}$vertex has the same form in all three models. This is due to the fact that the doubly-charged member of the triplet has the same electroweak quantum numbers in each model. However, because the $H_{5}^{ \pm}$of the Georgi-Machacek model is a mixture of the $\chi^{ \pm}$and $\xi^{ \pm}$states, which have $\left(T_{3}, Y\right)=(0, \pm 1)$ and $( \pm 1,0)$ respectively, this model does not have the same $W^{+} Z \Phi^{-}$ vertex as in the Littlest Higgs and Left-Right Symmetric models.

In principle, one could also use vector boson scattering to study the production of the $H_{1}^{0 \prime}$ singlet in the Georgi-Machacek model. From the Feynman rules in Table 3.1, it is evident that it couples more strongly to the $W$ and $Z$ bosons than the $H_{5}$ triplet does. Therefore, depending on its mass, the $H_{1}^{0 \prime}$ may be more easily observable in the $W^{+} W^{-}$and $Z Z$ channels. Production of the $H_{1}^{0 \prime}$ is also a potential background to the $H_{5}^{0}$ production processes of interest. However, for simplicity, the $H_{1}^{0 \prime}$ mass was assumed to be much larger than that of the $H_{5}$ triplet so that it does not contribute significantly to the $W^{+} W^{-}$and $Z Z$ scattering cross sections.

Hadronic decays of the vector bosons have the largest branching ratios. However, the QCD backgrounds to these final states are very large and difficult to disentangle from the signal. In contrast, the purely leptonic decays, with $W^{ \pm} \rightarrow \ell^{ \pm} \nu$ and $Z \rightarrow$ $\ell^{+} \ell^{-}(\ell=e, \mu)$, provide a much cleaner signal than hadronic decays, at the cost of a smaller branching ratio. These purely leptonic final states are often referred to 
as "gold-plated" modes. A final possibility is to consider semileptonic final states, sometimes referred to as "silver-plated" modes, which are intermediate in terms of branching ratios and clean final states. As expertise for reconstructing vector bosons in hadronic modes improves, these final states could prove to be useful for studying vector boson scattering. However, only the "gold-plated" leptonic final states are considered in this study.

MadGraph includes many diagrams with the same leptonic final state as the processes of interest. Therefore, having MadGraph calculate the full process including decays to final state leptons includes a very large number of non-resonant diagrams and takes considerable computing time. Instead, MadGraph was used to calculate the $p p \rightarrow \Phi+j j \rightarrow V V+j j$ processes and the vector bosons were subsequently decayed using the DECAY package of MadGraph. The resulting cross sections and distributions from both approaches were compared and were found to agree within the statistical uncertainties of the Monte Carlo.

As a final check of these results, the cross sections were calculated using the Effective Vector Boson Approximation [79] and the Goldstone Equivalence Theorem [80], and it was found that the two approaches were in reasonable agreement [81]. MadGraph includes a more complete set of Feynman diagrams that contributes to the final states under consideration and, more importantly, includes the spectator jets which are a crucial ingredient for reducing backgrounds.

\subsection{Backgrounds}

The transverse polarization vectors for the vector bosons are given by

$$
\begin{aligned}
& \epsilon_{T_{1}}^{\mu}=(0,1,0,0) \\
& \epsilon_{T_{2}}^{\mu}=(0,0,1,0)
\end{aligned}
$$


whereas the longitudinal polarization vectors are of the form

$$
\epsilon_{L}^{\mu} \sim \frac{p^{\mu}}{M_{V}} .
$$

As the latter is proportional to the momentum four-vector, $p^{\mu}$, at the high energies associated with $\mathrm{TeV}$ scale Higgs boson production at the LHC, the cross section for vector boson scattering is dominated by scattering of longitudinally polarized gauge bosons, $V_{L} V_{L} \rightarrow \Phi \rightarrow V_{L} V_{L}$, where $V=W^{ \pm}, Z$. Therefore, the signal process of interest is

$$
p p \rightarrow V_{L} V_{L} j j,
$$

with both vector bosons decaying leptonically. In general, the experimental signature consists of leptons in the central region of the detector with high transverse momentum $\left(p_{T}\right)$, two high- $p_{T}$ forward jets and low jet activity in the central region. Three types of backgrounds to this signal are considered, following the treatment of [68-70].

The first background is the irreducible electroweak (EW) background. It is similar to the signal process except that at least one of the vector bosons is transversely polarized:

$$
\begin{aligned}
& p p \rightarrow V_{T} V_{L} j j \\
& p p \rightarrow V_{T} V_{T} j j .
\end{aligned}
$$

This process is a background in the sense that its cross section is essentially insensitive to the new $\mathrm{TeV}$ scale physics under study. It is irreducible in the sense that the final state is virtually identical to the signal process, differing only by the polarization of the vector bosons, making this background difficult to reduce using simple kinematic cuts. In principle, if one could distinguish between the final state vector boson polarizations, this background could be considerably reduced. A recent paper by Han et al. argues 
that this can be done [82]. In any case, the cuts implemented in this study were found to reduce this background to a manageable level. The signal cross section for $\mathrm{TeV}$ scale Higgs boson production through vector boson scattering is therefore defined as

$$
\sigma_{\text {signal }}\left(p p \rightarrow V_{L} V_{L} j j\right) \equiv \sigma_{\text {total }}(p p \rightarrow V V j j)-\sigma_{S M}(p p \rightarrow V V j j),
$$

where $\sigma_{S M}(p p \rightarrow V V j j)$ is the cross section for the EW background process of the Standard Model, and $\sigma_{\text {total }}(p p \rightarrow V V j j)$ includes the contributions from the Higgs triplet model under consideration.

The second class of background processes are QCD backgrounds, which involve the production of two vector bosons plus additional jets:

$$
p p \rightarrow V V+n \text { jets, }
$$

where $n$ is the number of jets in the final state, typically with $n=2$. The 1 -jet background is also included because only one forward jet is tagged in this analysis. It is possible to tag two high- $p_{T}$ forward jets, but such double tagging has been shown to be too costly to the number of signal events [68]. Choosing to tag a single forward jet retains a larger fraction of the signal events in this analysis.

These backgrounds are calculated to $\mathcal{O}\left(\alpha^{2} \alpha_{s}^{n}\right)$, where $n=1$ or 2 , in contrast to the irreducible EW background, which is of order $\mathcal{O}\left(\alpha^{4}\right)$. Since the $V V$ scattering signal is a purely electroweak process with no colour exchange and relatively low jet activity in the central region, the jets arising in the QCD background are generally more central and have higher $p_{T}$ than the spectator jets from the signal events. Therefore, in addition to a forward jet tag, imposing a central jet veto to reject events with hard central jets is very useful in reducing the QCD background.

The third type of background under consideration includes top quarks in the 
final state, generally along with a vector boson and possibly an additional jet. In the $W^{+} W^{-}$channel, this background is top quark pair production via gluon fusion, which to lowest order is $\mathcal{O}\left(\alpha_{s}^{2}\right)$ :

$$
\begin{aligned}
& p p \rightarrow t \bar{t} \\
& p p \rightarrow t \bar{t} j
\end{aligned}
$$

In the $W^{ \pm} Z$ channel, this background is top quark pair production in association with a $Z$ boson, which to lowest order is $\mathcal{O}\left(\alpha \alpha_{s}^{2}\right)$ :

$$
\begin{aligned}
& p p \rightarrow t \bar{t} Z, \\
& p p \rightarrow t \bar{t} Z j .
\end{aligned}
$$

In the $W^{ \pm} W^{ \pm}$channel, this background is top quark pair production in association with a $W$ boson, which to lowest order is $\mathcal{O}\left(\alpha \alpha_{s}^{2}\right)$ :

$$
\begin{aligned}
& p p \rightarrow t \bar{t} W^{ \pm} \\
& p p \rightarrow t \bar{t} W^{ \pm} j .
\end{aligned}
$$

The top quark background is largest in the $W^{+} W^{-}$channel, as it is not suppressed by an additional factor of $\alpha$. These backgrounds arise from the leptonic decays of the vector boson, $V=W^{ \pm}$or $Z$, along with the $t$ and $\bar{t}$ decaying into real $W$ bosons, which then decay leptonically or hadronically. The resulting final state can have many leptons and jets that can mimic the signal. Fortunately, unlike the jets from the signal process, the jets from the $t$-quark background tend to emerge in the central region of the detector. Therefore, the $t$-quark background can also be suppressed by imposing a forward jet tag and a central jet veto. 
In the following subsections, a more detailed description of the backgrounds to each of the final states is given, along with the kinematic cuts that were used to reduce them to manageable levels.

\subsubsection{The $W^{ \pm} W^{ \pm}$Channel}

The Standard Model backgrounds to the $W^{ \pm} W^{ \pm}$channel are:

- EW Background:

$$
p p \rightarrow W^{ \pm} W^{ \pm} j j, \mathcal{O}\left(\alpha^{4}\right)
$$

- QCD Background:

$$
p p \rightarrow W^{ \pm} W^{ \pm} j j, \mathcal{O}\left(\alpha^{2} \alpha_{s}^{2}\right)
$$

- Top Quark Background:

$$
\begin{aligned}
& p p \rightarrow W^{ \pm} t \bar{t}, \\
& p p \rightarrow W^{ \pm} t \bar{t} j,
\end{aligned}
$$

with $t \rightarrow W b$.

- $W^{ \pm} Z$ Background:

$$
p p \rightarrow W^{ \pm} Z+n \text { jets }
$$

with the $W^{ \pm}$and $Z$ decaying leptonically, $W^{ \pm} Z \rightarrow \ell^{ \pm} \nu \ell^{+} \ell^{-}$. This process is a background to the $W^{ \pm} W^{ \pm}$signal if the $\ell^{\mp}$ from the $Z$ decay is not observed, due to the finite coverage of the electromagnetic calorimeter. Therefore, all 
the EW, QCD and $t$-quark backgrounds to the $W^{ \pm} Z$ channel are included as backgrounds to the $W^{ \pm} W^{ \pm}$channel.

The $W^{+} W^{+}$final state and the charge-conjugate $W^{-} W^{-}$final state are included to enhance the statistics in this channel. Note that the cross sections for the two chargeconjugate channels are not identical due to different parton distribution functions contributing to the different charge states.

For the $W^{ \pm} W^{ \pm}$channel, the leptonic decay mode of interest is $W^{ \pm} W^{ \pm} \rightarrow \ell^{ \pm} \nu \ell^{ \pm} \nu$, where $\ell=e, \mu$. Because the neutrinos in the final state escape the detector without being detected, their four-momenta, and hence the invariant mass of the $W$ boson pair, cannot be fully reconstructed. However, since the colliding partons are known to carry zero momentum in the transverse direction (perpendicular to the beam axis), the missing transverse momentum, $\not{p}_{T}$, can be determined using momentum conservation. Therefore, the cluster transverse mass of the $W W$ pair is considered $[70,83,84]$ rather than the $W W$ invariant mass distribution. In general, for a pair of vector bosons decaying leptonically, the cluster transverse mass is defined as

$$
M_{T}^{2}(V V)=\left[\left(E_{T}\right)_{l e p t o n s}+\mathbb{E}_{T}\right]^{2}-\left[\left(\vec{p}_{T}\right)_{l e p t o n s}+\vec{p}_{T}\right]^{2}
$$

The transverse energy is defined as $E_{T} \equiv \sqrt{M^{2}+p_{T}^{2}}$, where

$$
M^{2} \equiv g^{\mu \nu} p_{\mu} p_{\nu}=E^{2}-p_{x}^{2}-p_{y}^{2}-p_{z}^{2}
$$

is the invariant mass, and the transverse momentum is defined by

$$
p_{T}^{2} \equiv p_{x}^{2}+p_{y}^{2}
$$


with the proton beams assumed to travel along the $z$-axis. The missing transverse energy is defined by $\mathbb{E}_{T} \equiv\left|\vec{p}_{T}\right|$. Therefore, the $W W$ cluster transverse mass is given by

$$
M_{T}^{2}(W W)=\left[\sqrt{M^{2}(\ell \ell)+p_{T}^{2}(\ell \ell)}+\left|\vec{p}_{T}\right|\right]^{2}-\left[\vec{p}_{T}(\ell \ell)+\vec{p}_{T}\right]^{2}
$$

where $\vec{p}_{T}(\ell \ell)=\vec{p}_{T}\left(\ell_{1}\right)+\vec{p}_{T}\left(\ell_{2}\right)$. With this definition, the production of a doublycharged Higgs boson through $W^{ \pm} W^{ \pm}$scattering results in a broad peak in the $M_{T}(W W)$ distribution with an endpoint at approximately the resonance mass.

\subsubsection{The $W^{ \pm} Z$ Channel}

In the $W^{ \pm} Z$ channel, the following Standard Model backgrounds are considered:

- EW Background:

$$
p p \rightarrow W^{ \pm} Z j j, \mathcal{O}\left(\alpha^{4}\right)
$$

- QCD Background:

$$
\begin{aligned}
& p p \rightarrow W^{ \pm} Z j, \mathcal{O}\left(\alpha^{2} \alpha_{s}\right) \\
& p p \rightarrow W^{ \pm} Z j j, \mathcal{O}\left(\alpha^{2} \alpha_{s}^{2}\right)
\end{aligned}
$$

- Top Quark Background:

$$
\begin{aligned}
& p p \rightarrow Z t \bar{t}, \\
& p p \rightarrow Z t \bar{t} j,
\end{aligned}
$$

with $t \rightarrow W b$. 
Note that the $W^{+} Z$ final state and the charge-conjugate $W^{-} Z$ final state are considered to enhance the statistics in this channel.

From Equation 3.33, the $W Z$ cluster transverse mass is given by

$$
M_{T}^{2}(W Z)=\left[\sqrt{M^{2}(\ell \ell \ell)+p_{T}^{2}(\ell \ell \ell)}+\left|\vec{p}_{T}\right|\right]^{2}-\left[\vec{p}_{T}(\ell \ell \ell)+\vec{p}_{T}\right]^{2}
$$

The $M_{T}(W Z)$ distribution for singly-charged Higgs boson production through $W Z$ scattering shows a distinctive peak at the resonance mass.

\subsubsection{The $W^{+} W^{-}$Channel}

In the $W^{+} W^{-}$channel, the following Standard Model backgrounds are considered:

- EW Background:

$$
p p \rightarrow W^{+} W^{-} j j, \mathcal{O}\left(\alpha^{4}\right)
$$

- QCD Background:

$$
\begin{aligned}
& p p \rightarrow W^{+} W^{-} j, \mathcal{O}\left(\alpha^{2} \alpha_{s}\right) \\
& p p \rightarrow W^{+} W^{-} j j, \mathcal{O}\left(\alpha^{2} \alpha_{s}^{2}\right)
\end{aligned}
$$

- Top Quark Background:

$$
\begin{aligned}
& p p \rightarrow l \bar{t} \\
& p p \rightarrow t \bar{t} j
\end{aligned}
$$

with $t \rightarrow W b$.

The $W^{ \pm} Z$ scattering process may also be a background to the $W^{+} W^{-}$channel if the $\ell^{ \pm}$from the $Z$ decay is not detected. However, this is far less important than the 
large QCD and $t$-quark backgrounds to $W^{+} W^{-}$scattering, so it has been neglected.

As in the $W^{ \pm} W^{ \pm}$channel, the $W^{+} W^{-}$invariant mass cannot be fully reconstructed for leptonic final states, so the $W^{+} W^{-}$cluster transverse mass, also given by Equation 3.36, is used instead.

\subsubsection{The $Z Z$ Channel}

In the $Z Z$ channel, the following Standard Model backgrounds are considered:

- EW Background:

$$
p p \rightarrow Z Z j j, \mathcal{O}\left(\alpha^{4}\right)
$$

- QCD Background:

$$
\begin{aligned}
& p p \rightarrow Z Z j, \mathcal{O}\left(\alpha^{2} \alpha_{s}\right) \\
& p p \rightarrow Z Z j j, \mathcal{O}\left(\alpha^{2} \alpha_{s}^{2}\right)
\end{aligned}
$$

An advantage of the $Z Z \rightarrow 4 \ell$ decay mode is that the final state can be completely reconstructed and the $Z Z$ invariant mass distribution can be plotted.

\subsubsection{Kinematic Cuts}

The kinematic cuts used to reduce the Standard Model backgrounds to vector boson scattering are well established [68-71]. The cuts used in this analysis are summarized in Table 3.2 , where the rapidity, $y$, is defined as

$$
y=\frac{1}{2} \ln \left(\frac{E+p_{z}}{E-p_{z}}\right)
$$

Experimentalists commonly use pseudo rapidity, $\eta$, defined as $\eta=-\ln (\theta / 2)$, where $\theta$ is the angle between the beam axis and the direction of motion of the particle, 
Table 3.2: Leptonic cuts and jet cuts used in vector boson scattering to enhance the signal to background ratio. The central jet veto cuts are interpreted as: "Reject all events with jets having $|y|<y_{\max }$ and $p_{T}>p_{T_{\min } .}$ "

\begin{tabular}{|c|c|c|}
\hline Char & s & Jet \\
\hline & $\begin{array}{l}|y(\ell)|<2.5 \\
p_{T}\left(\ell_{1}\right)>200 \mathrm{GeV}, p_{T}\left(\ell_{2}\right)>50 \mathrm{GeV} \\
E_{T}>50 \mathrm{GeV} \\
\Delta p_{T}(\ell \ell)=\left|\vec{p}_{T}\left(\ell_{1}\right)-\vec{p}_{T}\left(\ell_{2}\right)\right|>300 \mathrm{GeV} \\
\Delta y(\ell \ell)=\left|y\left(\ell_{1}\right)-y\left(\ell_{2}\right)\right|<3 \\
M_{T}(W W)>550(800) \mathrm{GeV} \\
\quad \text { for } M_{\Phi}=1.0(1.5) \mathrm{TeV}\end{array}$ & $\begin{array}{l}3.0<\left|y\left(j_{\text {tag }}\right)\right|<5.0 \\
p_{T}\left(j_{\text {tag }}\right)>40 \mathrm{GeV} \\
E\left(j_{\text {tag }}\right)>500 \mathrm{GeV} \\
\left|y\left(j_{\text {veto }}\right)\right|<3.0 \\
p_{T}\left(j_{\text {veto }}\right)>100 \mathrm{GeV}\end{array}$ \\
\hline$W^{ \pm} Z$ & $\begin{array}{l}|y(\ell)|<2.5 \\
p_{T}\left(\ell_{1}\right)>150 \mathrm{GeV} \\
p_{T}\left(\ell_{2}\right)>50 \mathrm{GeV}, p_{T}\left(\ell_{3}\right)>50 \mathrm{GeV} \\
\mathbb{E}_{T}>50 \mathrm{GeV} \\
M_{T}(W Z)>900(1250) \mathrm{GeV} \\
\quad \text { for } M_{\Phi}=1.0(1.5) \mathrm{TeV}\end{array}$ & $\begin{array}{l}3.0<\left|y\left(j_{\text {tag }}\right)\right|<5.0 \\
p_{T}\left(j_{\text {tag }}\right)>40 \mathrm{GeV} \\
E\left(j_{\text {tag }}\right)>500 \mathrm{GeV} \\
\\
\left|y\left(j_{\text {veto }}\right)\right|<3.0 \\
p_{T}\left(j_{\text {veto }}\right)>100 \mathrm{GeV}\end{array}$ \\
\hline$W^{-}$ & $\begin{array}{l}|y(\ell)|<2.5 \\
p_{T}\left(\ell_{1}\right)>300 \mathrm{GeV}, p_{T}\left(\ell_{2}\right)>150 \mathrm{GeV} \\
\mathbb{E}_{T}>50 \mathrm{GeV} \\
\Delta p_{T}(\ell \ell)=\left|\vec{p}_{T}\left(\ell_{1}\right)-\vec{p}_{T}\left(\ell_{2}\right)\right|>500 \mathrm{GeV} \\
\Delta y(\ell \ell)=\left|y\left(\ell_{1}\right)-y\left(\ell_{2}\right)\right|<3 \\
M_{T}(W W)>700(1000) \mathrm{GeV} \\
\quad \text { for } M_{\Phi}=1.0(1.5) \mathrm{TeV} \\
\end{array}$ & $\begin{array}{l}3.0<\left|y\left(j_{\text {tag }}\right)\right|<5.0 \\
p_{T}\left(j_{\text {tag }}\right)>40 \mathrm{GeV} \\
E\left(j_{\text {tag }}\right)>800 \mathrm{GeV} \\
\left|y\left(j_{\text {veto }}\right)\right|<3.0 \\
p_{T}\left(j_{\text {veto }}\right)>60 \mathrm{GeV}\end{array}$ \\
\hline$Z Z$ & $\begin{array}{l}|y(\ell)|<2.5 \\
p_{T}(\ell)>70 \mathrm{GeV} \\
M(Z Z)>900(1250) \mathrm{GeV} \\
\quad \text { for } M_{\Phi}=1.0(1.5) \mathrm{TeV}\end{array}$ & $\begin{array}{l}3.0<\left|y\left(j_{\text {tag }}\right)\right|<5.0 \\
p_{T}\left(j_{\text {tag }}\right)>40 \mathrm{GeV} \\
E\left(j_{\text {tag }}\right)>500 \mathrm{GeV} \\
\text { no jet veto }\end{array}$ \\
\hline
\end{tabular}


$\cos \theta=p_{z} /|\vec{p}|$. Since these two quantities are approximately equal for $p \gg m$, the rapidity, $y$, is used in this analysis.

Cuts on the $p_{T}$ of all charged leptons have been imposed, along with cuts on the missing transverse energy $\left(\mathbb{E}_{T}\right)$ in the $W^{ \pm} Z$ channel. Back-to-back lepton cuts $\left(\Delta y(\ell \ell)\right.$ and $\left.\Delta p_{T}(\ell \ell)\right)$ have also been imposed in the $W^{ \pm} W^{ \pm}$and $W^{+} W^{-}$channels. It was assumed that the charge and flavour of the leptons can be determined so that the $W^{ \pm} W^{ \pm}$and $W^{+} W^{-}$channels could be distinguished. Rapidity cuts on the final state leptons of $|y(\ell)|<2.5$ and on the tag jet of $3.0<\left|y\left(j_{\text {tag }}\right)\right|<5.0$ have been imposed to take into account detector acceptances. Imposing a central jet veto was not necessary in the $Z Z$ channel, as this was found to further reduce the already small number of signal events. Note that the central jet veto cuts are to be interpreted as: "Reject all events with jets having $|y|<y_{\max }$ and $p_{T}>p_{T_{\min }}$ ". It should also be noted that, although a single energetic forward jet was tagged with high $p_{T}$, all jets are assumed to have $p_{T}>10 \mathrm{GeV}$ by default. This threshold is necessary for MadGraph to achieve stable results in its cross section calculations, and is also required to reconstruct these jets experimentally. These choices of cuts are found to be effective in improving the signal to background ratio.

\subsection{Results}

The MadGraph software package [78], version 4.2.6, was used to generate weighted signal and background events. The following Standard Model input parameters were used [77]: $G_{F}=1.16637 \times 10^{-5} \mathrm{GeV}^{-2}, M_{Z}=91.188 \mathrm{GeV}, \alpha\left(M_{Z}\right)=1 / 127.9$, $\alpha_{s}\left(M_{Z}\right)=0.118, M_{t}=171.2 \mathrm{GeV}$. A Standard Model Higgs boson mass of $M_{h}=120$ $\mathrm{GeV}$ was assumed and the CTEQ6M parton distribution functions [85] were used. The nominal LHC energy of $\sqrt{s}=14 \mathrm{TeV}$ was assumed.

In the Higgs triplet models under study, there are additional parameters in the 
Table 3.3: The partial widths of the Higgs triplet bosons for the models under study. The widths are in $\mathrm{GeV}$.

\begin{tabular}{|l|c|c|c|c|c|c|}
\hline Model: & \multicolumn{2}{|c|}{ Littlest Higgs } & \multicolumn{2}{c|}{ Georgi-Machacek } & \multicolumn{2}{c|}{ Left-Right Symmetric } \\
\hline$M_{\Phi}(\mathrm{TeV}):$ & 1.0 & 1.5 & 1.0 & 1.5 & 1.0 & 1.5 \\
\hline$\Gamma\left(\Phi^{ \pm \pm} \rightarrow W^{ \pm} W^{ \pm}\right):$ & 64 & 220 & 64 & 220 & 64 & 220 \\
$\Gamma\left(\Phi^{ \pm} \rightarrow W^{ \pm} Z\right):$ & 32 & 110 & 63 & 220 & 32 & 110 \\
$\Gamma\left(\Phi^{ \pm} \rightarrow W^{ \pm} h\right):$ & 42 & 150 & - & - & - & - \\
$\Gamma\left(\Phi^{0} \rightarrow W^{+} W^{-}\right):$ & - & - & 21 & 73 & 64 & 220 \\
$\Gamma\left(\Phi^{0} \rightarrow Z Z\right):$ & 31 & 110 & 42 & 150 & 126 & 440 \\
\hline
\end{tabular}

scalar sector that must be defined. For all models, triplet masses of $M_{\Phi}=1.0$ and $1.5 \mathrm{TeV}$ were used, along with a triplet vev of $v^{\prime}=39 \mathrm{GeV}$; as discussed in Section 3.2, this value of the triplet vev corresponds to the upper bound in the Georgi-Machacek model. In the Littlest Higgs model, the symmetry breaking scale, $f$, is an additional parameter that was set to $f=2 \mathrm{TeV}$. With these parameter values, the BRIDGE [86] package of MadGraph was used to calculate the scalar triplet decay widths for each of the models, and the resulting values are given in Table 3.3.

For the purpose of discussion, $W^{ \pm} Z$ scattering is considered as a representative case. The process is $p p \rightarrow W^{ \pm} Z j j$ with, for the "gold-plated" leptonic decay modes, $W^{ \pm} \rightarrow \ell^{ \pm} \nu$ and $Z \rightarrow \ell^{+} \ell^{-}(\ell=e, \mu)$. The experimental signature is given by two high- $p_{T}$ forward jets, along with three high- $p_{T}$ charged leptons in the central region of the detector. This is accompanied by a large amount of missing $p_{T}$ attributed to the undetected neutrino. The transverse mass distributions for each type of background along with the $W^{ \pm} Z$ signal before and after imposing the cuts of Table 3.2 are shown in Figure 3.2, for a triplet mass of $M_{\Phi}=1.0 \mathrm{TeV}$ and an integrated luminosity of $\mathcal{L}=100 \mathrm{fb}^{-1}$ at $\sqrt{s}=14 \mathrm{TeV}$. It can be seen from this figure that the cuts of Table 
3.2 reduce the irreducible EW background to a manageable level and substantially reduce the QCD and $t$-quark backgrounds.

All channels show qualitatively similar improvements in the signal to background ratio, so for the remaining channels, only the resulting mass distributions after imposing the kinematic cuts given in Table 3.2 are shown. The resulting cluster transverse mass distributions for the $W^{ \pm} W^{ \pm}, W^{ \pm} Z$, and $W^{+} W^{-}$channels, and the invariant mass distribution for the $Z Z$ channel are shown in Figures 3.3-3.6 for $M_{\Phi}=1.0$ and 1.5 TeV and an integrated luminosity of $\mathcal{L}=100 \mathrm{fb}^{-1}$ at $\sqrt{s}=14 \mathrm{TeV}$.

To better understand these plots, it is helpful to refer to the scalar triplet partial widths in these models, which are given in Table 3.3. Starting with the $W^{ \pm} W^{ \pm}$ channel, the transverse mass distributions for all three models shown in Figure 3.3 lie on top of each other because the doubly-charged member of the scalar triplet has the same $W^{+} W^{+} \Phi^{--}$vertex and the same decay width in each model. In contrast, the $W^{ \pm} Z$ channel, shown in Figure 3.4, exhibits differences between all three models. The peak width for the Littlest Higgs model signal is larger than that of the LeftRight Symmetric model signal because, although they have the same $W^{+} Z \Phi^{-}$vertex, the $\Phi^{ \pm}$of the Littlest Higgs model can also decay to $W^{ \pm} h$, resulting in a larger $\Phi^{ \pm}$ width. Furthermore, although the $H_{5}^{ \pm}$of the Georgi-Machacek model can only decay to $W^{ \pm} Z$, as discussed previously, the $W^{+} Z H_{5}^{-}$coupling is larger by a factor of $\sqrt{2}$ compared to the $W^{+} Z \Delta_{L}^{-}$coupling in the Left-Right Symmetric model, resulting in a $H_{5}^{ \pm}$width that is a factor of two larger than the $\Delta_{L}^{ \pm}$width and also a larger production cross section. Finally, the differential cross sections in the $Z Z$ channel shown in Figure 3.6 differ quite significantly due to the different $\Phi^{0} Z Z$ couplings and $\Phi^{0}$ widths. The width of the neutral member of the scalar triplet is smallest in the Littlest Higgs model, partially due to the absence of the $\Phi^{0} \rightarrow W^{+} W^{-}$decay mode at leading order in this model, but also due to the value of the $\Phi^{0} Z Z$ coupling. In 

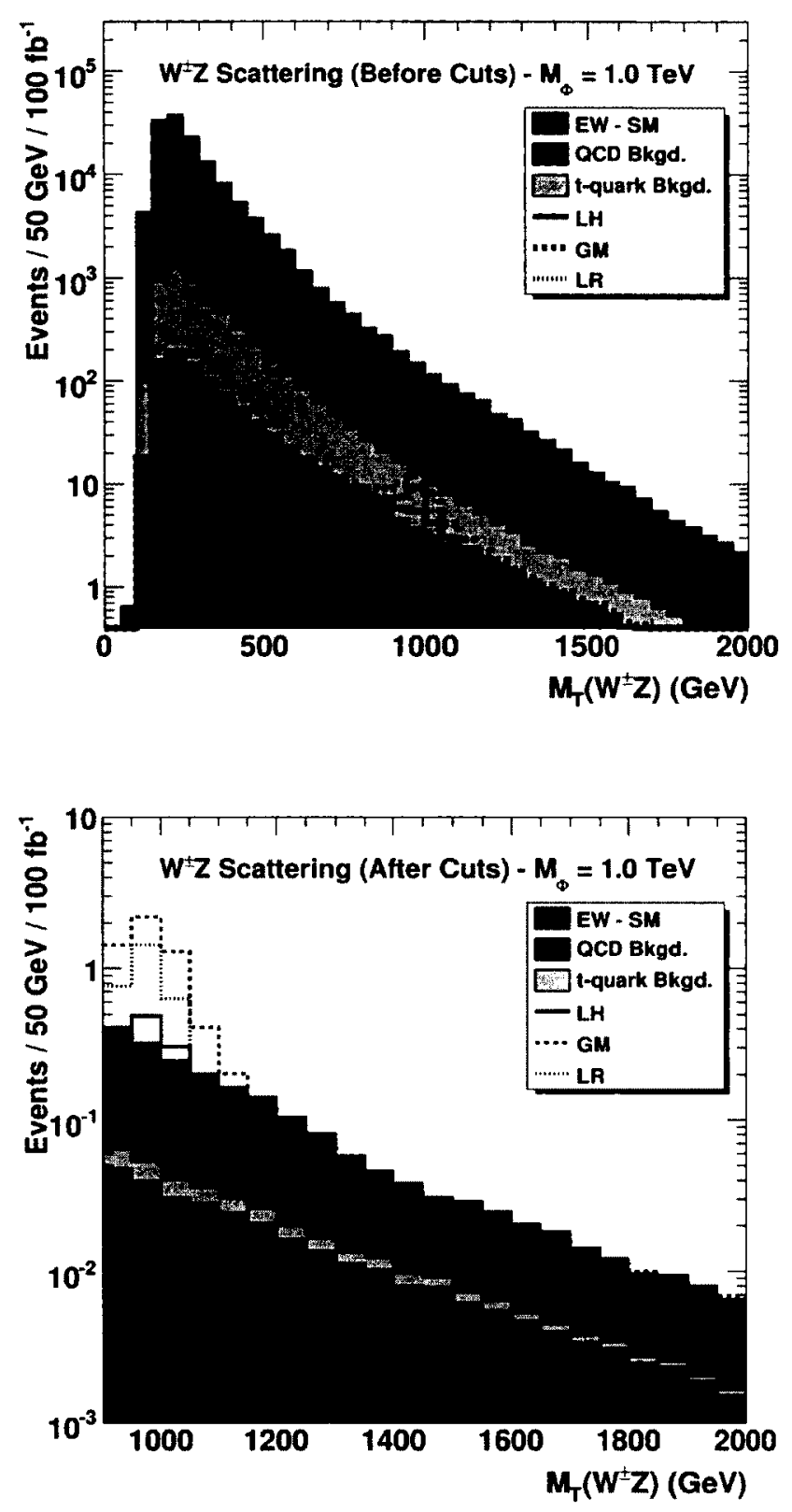

Figure 3.2: The transverse mass distributions for the $W^{ \pm} Z$ signals of the Littlest Higgs (LH), Georgi-Machacek (GM) and Left-Right Symmetric (LR) models, along with the backgrounds, before and after imposing the cuts of Table 3.2. A Higgs triplet mass of $M_{\Phi}=1.0 \mathrm{TeV}$ was used, along with a triplet vev of $v^{\prime}=39 \mathrm{GeV}$, assuming an integrated luminosity of $\mathcal{L}=100 \mathrm{fb}^{-1}$ at $\sqrt{s}=14 \mathrm{TeV}$. Note that the backgrounds are stacked, whereas the signal lines are not. It can be seen that the backgrounds are reduced and a relatively large fraction of the signal events remain. The horizontal scale was enlarged in the bottom figure to more clearly show the differences in the signal lines of each model after imposing cuts. 

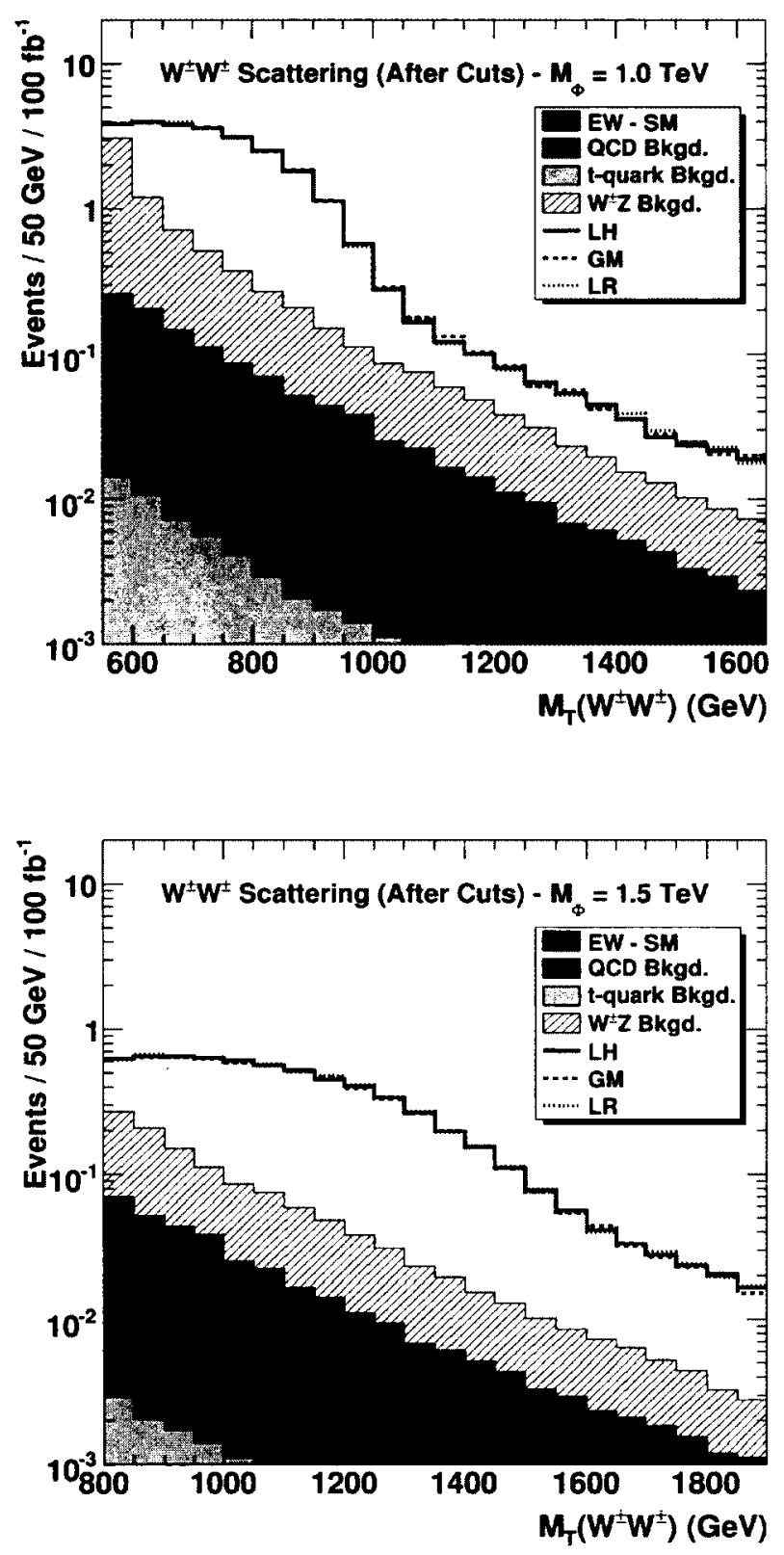

Figure 3.3: The transverse mass distributions for $W^{ \pm} W^{ \pm}$scattering in the Littlest Higgs (LH), Georgi-Machacek (GM) and Left-Right Symmetric (LR) models, along with the backgrounds, after imposing the cuts of Table 3.2. Higgs triplet masses of $M_{\Phi}=1.0$ and $1.5 \mathrm{TeV}$ were used, along with a triplet vev of $v^{\prime}=39 \mathrm{GeV}$, assuming an integrated luminosity of $\mathcal{L}=100 \mathrm{fb}^{-1}$ at $\sqrt{s}=14 \mathrm{TeV}$. It should be noted that the backgrounds are stacked, whereas the signal lines are not. 

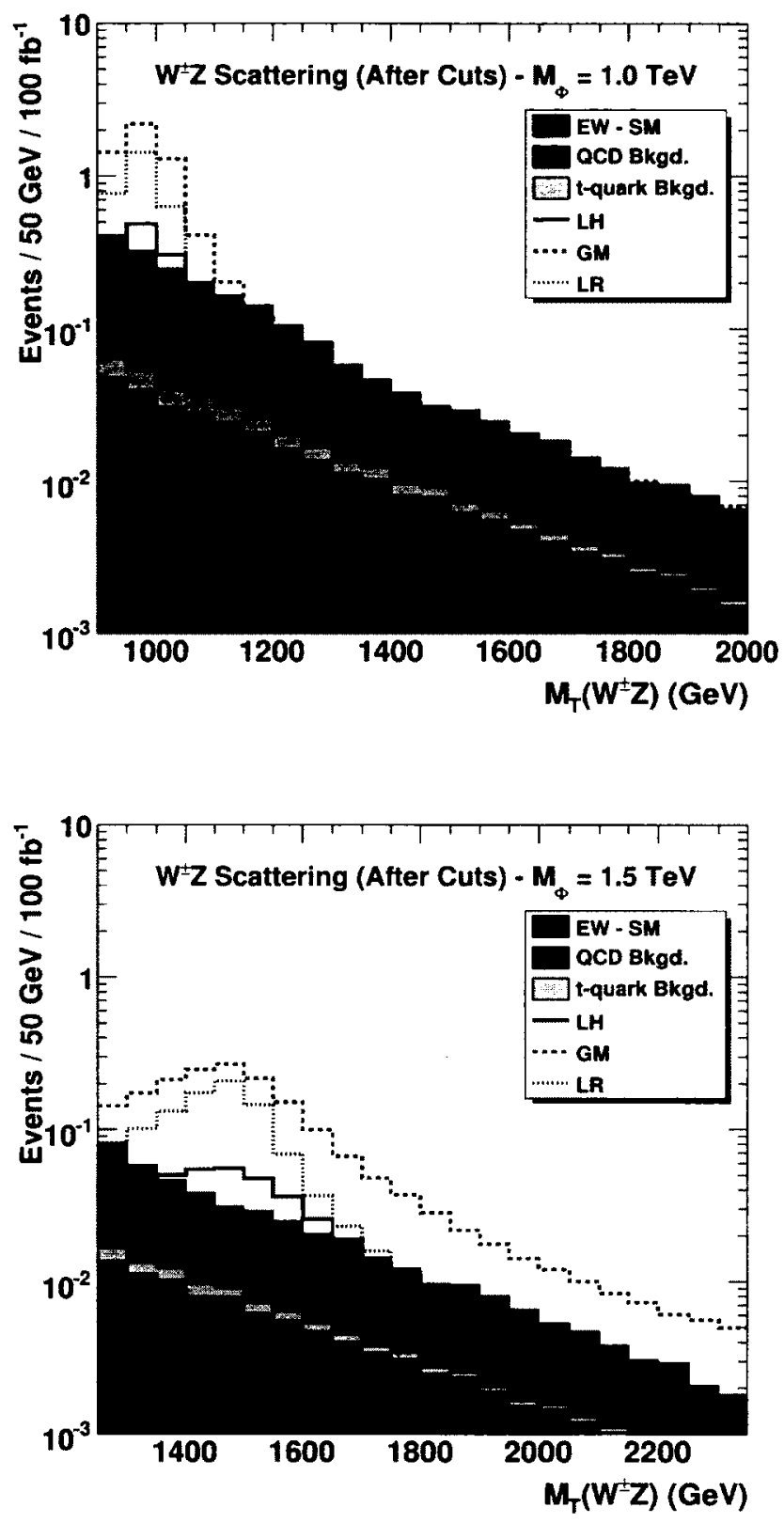

Figure 3.4: The transverse mass distributions for $W^{ \pm} Z$ scattering in the Littlest Higgs (LH), Georgi-Machacek (GM) and Left-Right Symmetric (LR) models, along with the backgrounds, after imposing the cuts of Table 3.2. Higgs triplet masses of $M_{\Phi}=1.0$ and $1.5 \mathrm{TeV}$ were used, along with a triplet vev of $v^{\prime}=39 \mathrm{GeV}$, assuming an integrated luminosity of $\mathcal{L}=100 \mathrm{fb}^{-1}$ at $\sqrt{s}=14 \mathrm{TeV}$. It should be noted that the backgrounds are stacked, whereas the signal lines are not. 

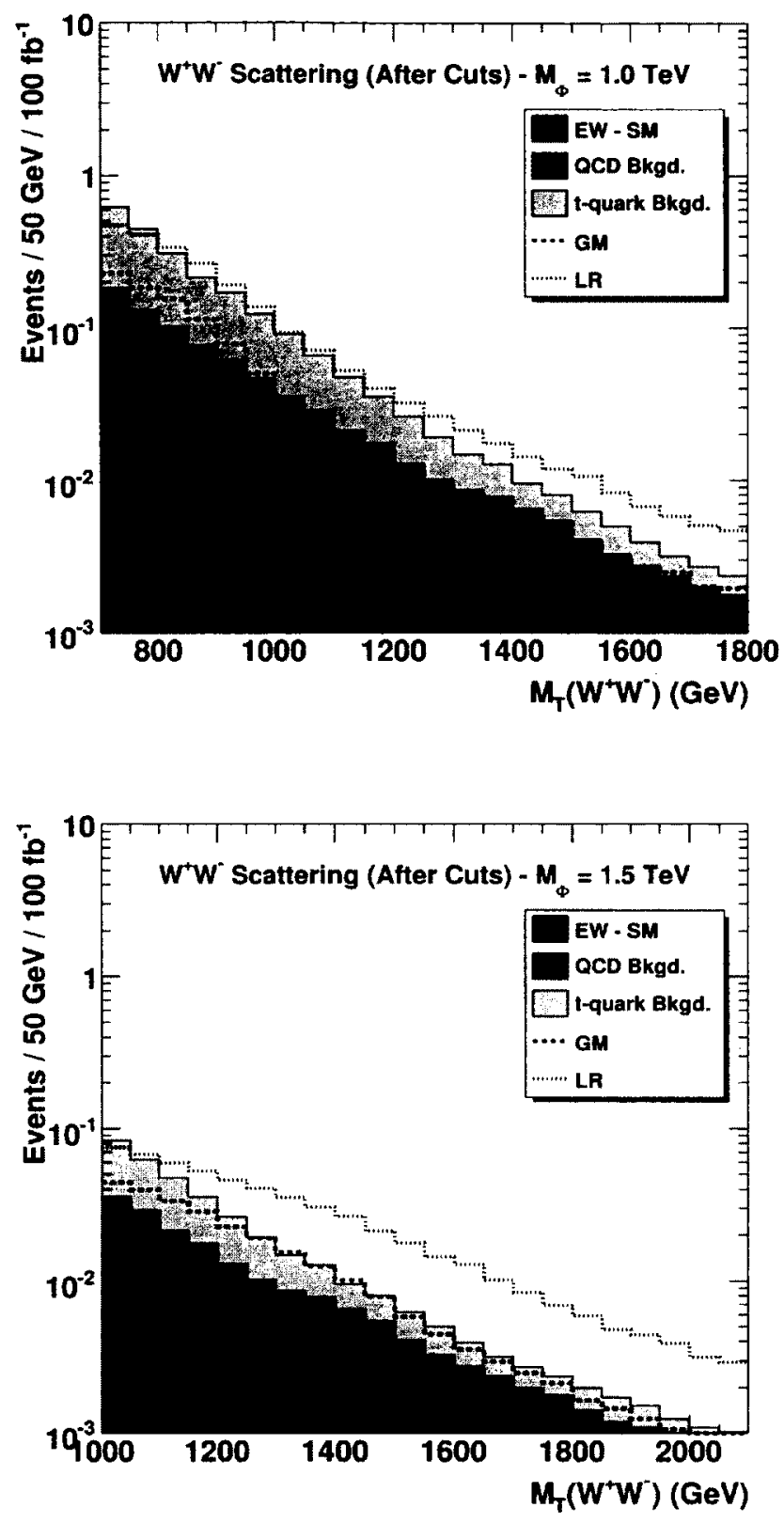

Figure 3.5: The transverse mass distributions for $W^{+} W^{-}$scattering in the GeorgiMachacek (GM) and Left-Right Symmetric (LR) models (this signal does not exist in the Littlest Higgs model at lowest order), along with the backgrounds, after imposing the cuts of Table 3.2. Higgs triplet masses of $M_{\Phi}=1.0$ and $1.5 \mathrm{TeV}$ were used, along with a triplet vev of $v^{\prime}=39 \mathrm{GeV}$, assuming an integrated luminosity of $\mathcal{L}=100 \mathrm{fb}^{-1}$ at $\sqrt{s}=14 \mathrm{TeV}$. It should be noted that the backgrounds are stacked, whereas the signal lines are not. 

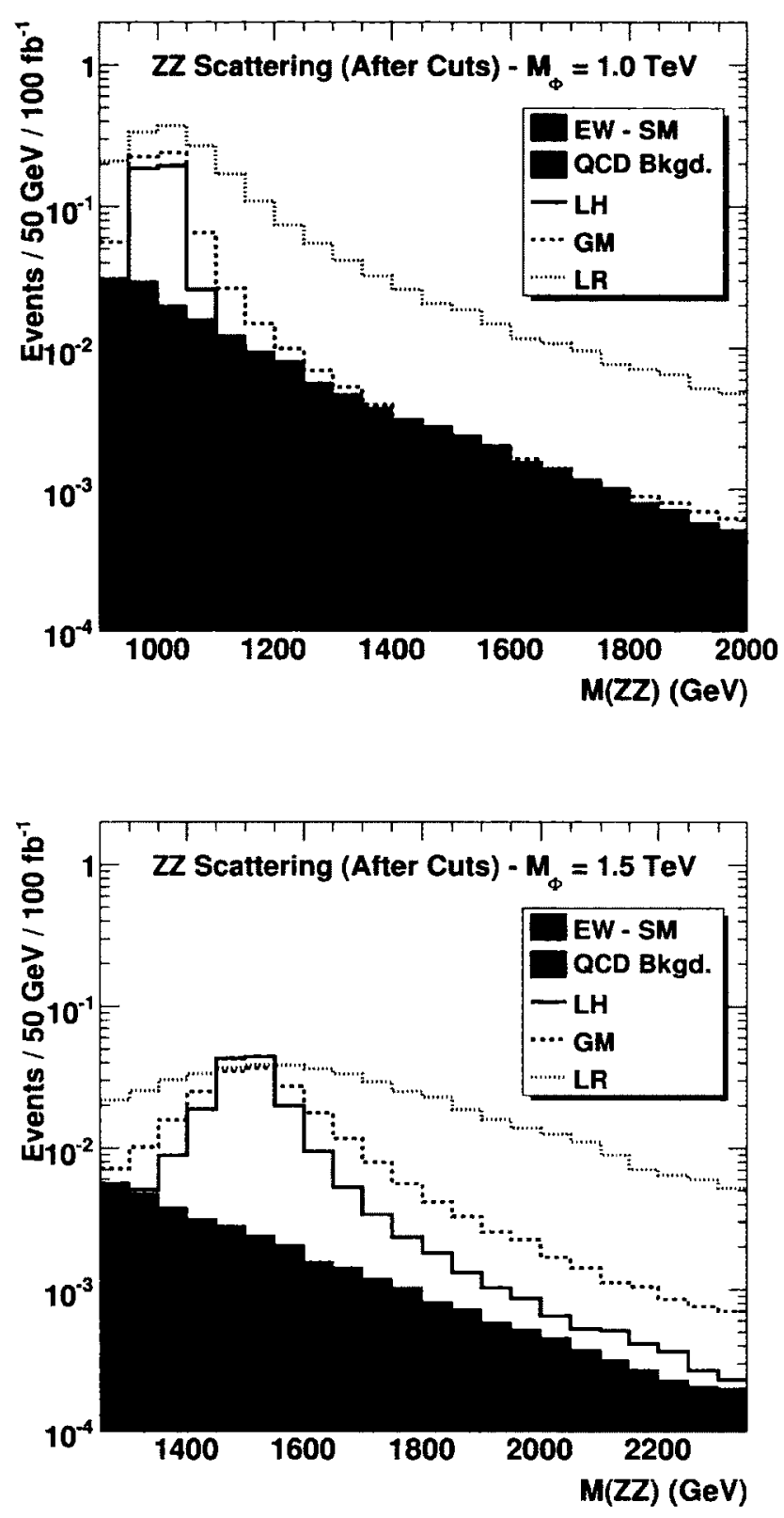

Figure 3.6: The invariant mass distributions for $Z Z$ scattering in the Littlest Higgs (LH), Georgi-Machacek (GM) and Left-Right Symmetric (LR) models, along with the backgrounds, after imposing the cuts of Table 3.2. Higgs triplet masses of $M_{\Phi}=1.0$ and $1.5 \mathrm{TeV}$ were used, along with a triplet vev of $v^{\prime}=39 \mathrm{GeV}$, assuming an integrated luminosity of $\mathcal{L}=100 \mathrm{fb}^{-1}$ at $\sqrt{s}=14 \mathrm{TeV}$. It should be noted that the backgrounds are stacked, whereas the signal lines are not. As the efficiency for reconstructing four leptons is expected to be $\sim 95 \%$ for electrons and even higher for muons [87], this was not taken into account in this analysis. 
both the Georgi-Machacek and Left-Right Symmetric models, the $\Phi^{0}$ can also decay to $W^{+} W^{-}$, which increases the total width. These effects are not so apparent in the $W^{+} W^{-}$channel because the signal is more deeply buried in the background.

More quantitatively, Tables 3.4-3.7 list the cross sections for the signal and backgrounds after imposing the cuts of Table 3.2. The signal cross sections for the Littlest Higgs model, the Georgi-Machacek model, and the Left-Right Symmetric model are shown for scalar triplet masses of $M_{\Phi}=1.0$ and $1.5 \mathrm{TeV}$. The rows labelled as EWSM give the Standard Model cross section, assuming a Higgs mass of $M_{h}=120 \mathrm{GeV}$, which is denoted in the text as the irreducible EW background. The rows labelled as Signal-LH, Signal-GM, and Signal-LR are the difference between the EW-SM cross section and the EW-LH, EW-GM, and EW-LR cross sections respectively, as defined by Equation 3.20 .

The observability of a Higgs triplet signal in a $V V$ channel is determined by the relative size of the signal to background, and by the assumed integrated luminosity. So, for example, in the Littlest Higgs model with $M_{\Phi}=1.0 \mathrm{TeV}$ and $v^{\prime}=39 \mathrm{GeV}$, the cross section after cuts in the $W^{ \pm} W^{ \pm}$channel is $0.244 \mathrm{fb}$, compared to the cross section of the combined backgrounds of $0.0798 \mathrm{fb}$. Assuming $100 \mathrm{fb}^{-1}$, this would result in roughly a $9 \sigma$ effect. One could invert this and ask what luminosity would be required to produce a $5 \sigma$ discovery (i.e. $S / \sqrt{B} \geq 5$ ). For this example, the luminosity required would be $34 \mathrm{fb}^{-1}$, resulting in $\sim 8$ signal events. To ensure that a substantial number of signal events are observed, a second criteria is imposed, requiring that there must be at least 10 signal events, resulting in a required integrated luminosity of $41 \mathrm{fb}^{-1}$. Following this procedure, Table 3.8 lists the integrated luminosities required to produce a $5 \sigma$ signal with at least 10 signal events in each model for triplet masses of 1.0 and $1.5 \mathrm{TeV}$.

The early years of LHC running are expected to produce $\sim 10 \mathrm{fb}^{-1} /$ year. It is 
Table 3.4: LHC cross sections (in $\mathrm{fb}$ ) for $W^{ \pm} W^{ \pm}$scattering with leptonic decays using the cuts listed in Table 3.2. The cross sections are shown for the three models under study, using a triplet vev of $v^{\prime}=39 \mathrm{GeV}$ and Higgs triplet masses of $M_{\Phi}=1.0$ $\mathrm{TeV}$ and $M_{\Phi}=1.5 \mathrm{TeV}$. The cross section values from MadGraph have statistical uncertainties of $\sim 1 \%$.

\begin{tabular}{|c|c|c|c|c|}
\hline \multicolumn{5}{|c|}{$\mathbf{W}^{ \pm} \mathbf{W}^{ \pm}$} \\
\hline$M_{\Phi}(\mathrm{TeV})$ & Process & Leptonic Cuts & + Jet Tag & + Jet Veto \\
\hline \multirow{11}{*}{1.0} & $E W-L H$ & $9.39 \times 10^{-1}$ & $3.03 \times 10^{-1}$ & $2.55 \times 10^{-1}$ \\
\hline & EW - GM & $9.41 \times 10^{-1}$ & $3.06 \times 10^{-1}$ & $2.56 \times 10^{-1}$ \\
\hline & EW - LR & $9.36 \times 10^{-1}$ & $3.04 \times 10^{-1}$ & $2.56 \times 10^{-1}$ \\
\hline & $\mathrm{EW}-\mathrm{SM}$ & $2.01 \times 10^{-1}$ & $1.57 \times 10^{-2}$ & $1.06 \times 10^{-2}$ \\
\hline & QCD Bkgd. & $1.16 \times 10^{-1}$ & $7.90 \times 10^{-4}$ & $4.22 \times 10^{-4}$ \\
\hline & $t$-quark Bkgd. & $4.42 \times 10^{-1}$ & $8.15 \times 10^{-4}$ & $5.52 \times 10^{-4}$ \\
\hline & $W^{ \pm} Z$ Bkgd. & 5.75 & $8.58 \times 10^{-2}$ & $6.82 \times 10^{-2}$ \\
\hline & Total Bkgd. & 6.51 & $1.03 \times 10^{-1}$ & $7.98 \times 10^{-2}$ \\
\hline & Signal - LH & $7.38 \times 10^{-1}$ & $2.87 \times 10^{-1}$ & $2.44 \times 10^{-1}$ \\
\hline & Signal - GM & $7.40 \times 10^{-1}$ & $2.90 \times 10^{-1}$ & $2.45 \times 10^{-1}$ \\
\hline & Signal - LR & $7.35 \times 10^{-1}$ & $2.88 \times 10^{-1}$ & $2.45 \times 10^{-1}$ \\
\hline \multirow{11}{*}{1.5} & EW - LH & $2.92 \times 10^{-1}$ & $1.05 \times 10^{-1}$ & $8.85 \times 10^{-2}$ \\
\hline & EW - GM & $2.88 \times 10^{-1}$ & $1.03 \times 10^{-1}$ & $8.75 \times 10^{-2}$ \\
\hline & EW - LR & $2.91 \times 10^{-1}$ & $1.04 \times 10^{-1}$ & $8.87 \times 10^{-2}$ \\
\hline & EW - SM & $6.48 \times 10^{-2}$ & $4.91 \times 10^{-3}$ & $3.42 \times 10^{-3}$ \\
\hline & QCD Bkgd. & $4.51 \times 10^{-2}$ & $2.47 \times 10^{-4}$ & $1.67 \times 10^{-4}$ \\
\hline & $t$-quark Bkgd. & $1.29 \times 10^{-1}$ & $1.38 \times 10^{-4}$ & $7.33 \times 10^{-5}$ \\
\hline & $W^{ \pm} Z$ Bkgd. & 1.45 & $1.91 \times 10^{-2}$ & $1.67 \times 10^{-2}$ \\
\hline & Total Bkgd. & 1.69 & $2.44 \times 10^{-2}$ & $2.04 \times 10^{-2}$ \\
\hline & Signal - LH & $2.27 \times 10^{-1}$ & $1.00 \times 10^{-1}$ & $8.51 \times 10^{-2}$ \\
\hline & Signal - GM & $2.23 \times 10^{-1}$ & $0.98 \times 10^{-1}$ & $8.41 \times 10^{-2}$ \\
\hline & Signal - LR & $2.26 \times 10^{-1}$ & $0.99 \times 10^{-1}$ & $8.53 \times 10^{-2}$ \\
\hline
\end{tabular}


Table 3.5: LHC cross sections (in $\mathrm{fb}$ ) for $W^{ \pm} Z$ scattering with leptonic decays using the cuts listed in Table 3.2. The cross sections are shown for the three models under study, using a triplet vev of $v^{\prime}=39 \mathrm{GeV}$ and Higgs triplet masses of $M_{\Phi}=1.0$ $\mathrm{TeV}$ and $M_{\Phi}=1.5 \mathrm{TeV}$. The cross section values from MadGraph have statistical uncertainties of $\sim 1 \%$.

\begin{tabular}{|c|c|c|c|c|}
\hline \multicolumn{5}{|c|}{$\mathbf{W}^{ \pm} \mathbf{Z}$} \\
\hline$M_{\Phi}(\mathrm{TeV})$ & Process & Leptonic Cuts & + Jet Tag & + Jet Veto \\
\hline \multirow{10}{*}{1.0} & EW - LH & $9.50 \times 10^{-2}$ & $1.95 \times 10^{-2}$ & $1.56 \times 10^{-2}$ \\
\hline & EW - GM & $2.44 \times 10^{-1}$ & $7.46 \times 10^{-2}$ & $6.15 \times 10^{-2}$ \\
\hline & $E W-L R$ & $1.51 \times 10^{-1}$ & $4.01 \times 10^{-2}$ & $3.28 \times 10^{-2}$ \\
\hline & EW - SM & $5.47 \times 10^{-2}$ & $4.47 \times 10^{-3}$ & $3.03 \times 10^{-3}$ \\
\hline & QCD Bkgd. & 1.60 & $1.96 \times 10^{-2}$ & $1.72 \times 10^{-2}$ \\
\hline & $t$-quark Bkgd. & $2.54 \times 10^{-1}$ & $1.02 \times 10^{-3}$ & $7.30 \times 10^{-4}$ \\
\hline & Total Bkgd. & 1.91 & $2.51 \times 10^{-2}$ & $2.10 \times 10^{-2}$ \\
\hline & Signal - LH & $4.03 \times 10^{-2}$ & $1.50 \times 10^{-2}$ & $1.26 \times 10^{-2}$ \\
\hline & Signal - GM & $1.89 \times 10^{-1}$ & $7.01 \times 10^{-2}$ & $5.85 \times 10^{-2}$ \\
\hline & Signal - LR & $0.96 \times 10^{-1}$ & $3.56 \times 10^{-2}$ & $2.98 \times 10^{-2}$ \\
\hline \multirow{10}{*}{1.5} & EW - LH & $3.12 \times 10^{-2}$ & $6.84 \times 10^{-3}$ & $5.48 \times 10^{-3}$ \\
\hline & EW - GM & $7.86 \times 10^{-2}$ & $2.66 \times 10^{-2}$ & $2.22 \times 10^{-2}$ \\
\hline & EW - LR & $5.13 \times 10^{-2}$ & $1.51 \times 10^{-2}$ & $1.25 \times 10^{-2}$ \\
\hline & EW - SM & $1.87 \times 10^{-2}$ & $1.60 \times 10^{-3}$ & $1.06 \times 10^{-3}$ \\
\hline & QCD Bkgd. & $3.34 \times 10^{-1}$ & $2.60 \times 10^{-3}$ & $2.25 \times 10^{-3}$ \\
\hline & $t$-quark Bkgd. & $5.62 \times 10^{-2}$ & $1.07 \times 10^{-4}$ & $8.93 \times 10^{-5}$ \\
\hline & Total Bkgd. & $4.09 \times 10^{-1}$ & $4.31 \times 10^{-3}$ & $3.40 \times 10^{-3}$ \\
\hline & Signal - LH & $1.25 \times 10^{-2}$ & $5.24 \times 10^{-3}$ & $4.42 \times 10^{-3}$ \\
\hline & Signal - GM & $5.99 \times 10^{-2}$ & $2.50 \times 10^{-2}$ & $2.11 \times 10^{-2}$ \\
\hline & Signal - LR & $3.26 \times 10^{-2}$ & $1.35 \times 10^{-2}$ & $1.14 \times 10^{-2}$ \\
\hline
\end{tabular}


Table 3.6: LHC cross sections (in fb) for $W^{+} W^{-}$scattering with leptonic decays using the cuts listed in Table 3.2. The cross sections are shown for the three models under study, using a triplet vev of $v^{\prime}=39 \mathrm{GeV}$ and Higgs triplet masses of $M_{\Phi}=1.0$ $\mathrm{TeV}$ and $M_{\Phi}=1.5 \mathrm{TeV}$. The cross section values from MadGraph have statistical uncertainties of $\sim 1 \%$. Note that the $W^{+} W^{-}$scattering signal does not occur in the Littlest Higgs model.

\begin{tabular}{|c|c|c|c|c|}
\hline \multicolumn{5}{|c|}{$\mathbf{W}^{+} \mathbf{W}^{-}$} \\
\hline$M_{\Phi}(\mathrm{TeV})$ & Process & Leptonic Cuts & + Jet Tag & + Jet Veto \\
\hline \multirow{10}{*}{1.0} & EW - LH & - & - & - \\
\hline & EW - GM & $1.62 \times 10^{-1}$ & $2.08 \times 10^{-2}$ & $9.91 \times 10^{-3}$ \\
\hline & EW - LR & $2.35 \times 10^{-1}$ & $4.63 \times 10^{-2}$ & $2.28 \times 10^{-2}$ \\
\hline & EW - SM & $1.32 \times 10^{-1}$ & $1.07 \times 10^{-2}$ & $4.75 \times 10^{-3}$ \\
\hline & QCD Bkgd. & 4.72 & $1.61 \times 10^{-2}$ & $4.29 \times 10^{-3}$ \\
\hline & $t$-quark Bkgd. & $5.70 \times 10^{1}$ & $5.16 \times 10^{-2}$ & $1.48 \times 10^{-2}$ \\
\hline & Total Bkgd. & $6.18 \times 10^{1}$ & $7.84 \times 10^{-2}$ & $2.38 \times 10^{-2}$ \\
\hline & Signal - LH & & - & - \\
\hline & Signal - GM & $0.30 \times 10^{-1}$ & $1.01 \times 10^{-2}$ & $5.16 \times 10^{-3}$ \\
\hline & Signal - LR & $1.03 \times 10^{-1}$ & $3.56 \times 10^{-2}$ & $1.80 \times 10^{-2}$ \\
\hline \multirow{10}{*}{1.5} & EW - LH & - & - & - \\
\hline & EW - GM & $7.28 \times 10^{-2}$ & $9.20 \times 10^{-3}$ & $4.35 \times 10^{-3}$ \\
\hline & EW - LR & $9.70 \times 10^{-2}$ & $1.84 \times 10^{-2}$ & $9.23 \times 10^{-3}$ \\
\hline & EW - SM & $5.98 \times 10^{-2}$ & $4.32 \times 10^{-3}$ & $1.79 \times 10^{-3}$ \\
\hline & QCD Bkgd. & $9.18 \times 10^{-1}$ & $3.13 \times 10^{-3}$ & $8.34 \times 10^{-4}$ \\
\hline & $t$-quark Bkgd. & $1.28 \times 10^{1}$ & $1.18 \times 10^{-2}$ & $3.99 \times 10^{-3}$ \\
\hline & Total Bkgd. & $1.38 \times 10^{1}$ & $1.92 \times 10^{-2}$ & $6.61 \times 10^{-3}$ \\
\hline & Signal - LH & - & & - \\
\hline & Signal - GM & $1.30 \times 10^{-2}$ & $4.88 \times 10^{-3}$ & $2.56 \times 10^{-3}$ \\
\hline & Signal - LR & $3.72 \times 10^{-2}$ & $1.41 \times 10^{-2}$ & $7.44 \times 10^{-3}$ \\
\hline
\end{tabular}


Table 3.7: LHC cross sections (in $\mathrm{fb}$ ) for $Z Z$ scattering with decays to four leptons using the cuts listed in Table 3.2. The cross sections are shown for the three models under study, using a triplet vev of $v^{\prime}=39 \mathrm{GeV}$ and Higgs triplet masses of $M_{\Phi}=1.0$ $\mathrm{TeV}$ and $M_{\Phi}=1.5 \mathrm{TeV}$. The cross section values from MadGraph have statistical uncertainties of $\sim 1 \%$. Note that a central jet veto was not needed in the $Z Z$ channel as this was found to reduce the already small number of signal events.

\begin{tabular}{|c|l|l|l|}
\hline \multicolumn{3}{|c|}{ ZZ } \\
\hline$M_{\Phi}(\mathrm{TeV})$ & Process & Leptonic Cuts & + Jet Tag \\
\hline \hline \multirow{6}{*}{1.0} & EW - LH & $1.59 \times 10^{-2}$ & $4.67 \times 10^{-3}$ \\
& EW - GM & $2.12 \times 10^{-2}$ & $6.77 \times 10^{-3}$ \\
& EW - LR & $5.28 \times 10^{-2}$ & $1.86 \times 10^{-2}$ \\
& EW - SM & $3.20 \times 10^{-3}$ & $1.83 \times 10^{-4}$ \\
& QCD Bkgd. & $8.00 \times 10^{-2}$ & $1.50 \times 10^{-3}$ \\
& Total Bkgd. & $8.32 \times 10^{-2}$ & $1.68 \times 10^{-3}$ \\
& Signal - LH & $1.27 \times 10^{-2}$ & $4.49 \times 10^{-3}$ \\
& Signal - GM & $1.80 \times 10^{-2}$ & $6.59 \times 10^{-3}$ \\
& Signal - LR & $4.96 \times 10^{-2}$ & $1.84 \times 10^{-2}$ \\
\hline \hline \multirow{6}{*}{1.5} & EW - LH & $5.58 \times 10^{-3}$ & $1.78 \times 10^{-3}$ \\
& EW - GM & $7.01 \times 10^{-3}$ & $2.38 \times 10^{-3}$ \\
& EW - LR & $1.51 \times 10^{-2}$ & $5.87 \times 10^{-3}$ \\
& EW - SM & $1.30 \times 10^{-3}$ & $4.80 \times 10^{-5}$ \\
& QCD Bkgd. & $1.67 \times 10^{-2}$ & $3.49 \times 10^{-4}$ \\
& Total Bkgd. & $1.80 \times 10^{-2}$ & $3.97 \times 10^{-4}$ \\
& Signal - LH & $4.28 \times 10^{-3}$ & $1.73 \times 10^{-3}$ \\
& Signal - GM & $5.71 \times 10^{-3}$ & $2.33 \times 10^{-3}$ \\
& Signal - LR & $1.38 \times 10^{-2}$ & $5.82 \times 10^{-3}$ \\
\hline
\end{tabular}


Table 3.8: The integrated luminosity (in $\mathrm{fb}^{-1}$ ) required for a statistical significance of $S / \sqrt{B} \geq 5$ and at least 10 signal events, after the cuts of Table 3.2 have been imposed. These results were obtained for a triplet vev of $v^{\prime}=39 \mathrm{GeV}$, using the cross section values listed in Tables 3.4-3.7.

\begin{tabular}{|l|c|c|c|c|c|c|}
\hline Model: & \multicolumn{2}{|c|}{ Littlest Higgs } & \multicolumn{2}{c|}{ Georgi-Machacek } & \multicolumn{2}{|c|}{ Left-Right Symmetric } \\
\hline$M_{\Phi}(\mathrm{TeV}):$ & 1.0 & 1.5 & 1.0 & 1.5 & 1.0 & 1.5 \\
\hline$W^{ \pm} W^{ \pm}:$ & 41 & 118 & 41 & 119 & 41 & 117 \\
\hline$W^{ \pm} Z:$ & 3300 & 4350 & 171 & 474 & 591 & 877 \\
\hline$W^{+} W^{-}:$ & - & - & 22300 & 25200 & 1840 & 2980 \\
\hline$Z Z:$ & 2230 & 5780 & 1520 & 4290 & 543 & 1720 \\
\hline
\end{tabular}

therefore unlikely that a heavy Higgs triplet would be observed at the LHC in this period of time. Once design luminosity is achieved, it is expected that $\sim 100 \mathrm{fb}^{-1}$ will be produced per year. In this case it should be possible to observe a doubly-charged Higgs triplet in any of these models with the assumed parameter values. In the $W^{ \pm} Z$ channel, it seems that only the charged scalar in the Georgi-Machacek model could be observed. The $W^{+} W^{-}$channel is the least promising for observing a Higgs triplet due to the large QCD and t-quark backgrounds in this channel.

The values in Tables 3.4-3.7 and Table 3.8 were obtained assuming a triplet vev of $v^{\prime}=39 \mathrm{GeV}$, which is the upper bound in the Georgi-Machacek model. However, in the Littlest Higgs model this parameter is constrained to $v^{\prime} \lesssim 4 \mathrm{GeV}$, while in the Left-Right Symmetric model the constraint is $v^{\prime} \lesssim 2 \mathrm{GeV}$. The Higgs triplet production cross section is proportional to $v^{\prime 2}$, so, with these upper limits, the cross sections would be reduced by a factor of roughly $10^{-2}$ and $2 \times 10^{-3}$ for the Littlest Higgs and Left-Right Symmetric models respectively. With the resulting cross section values, it is clear that to observe any of the $\mathrm{TeV}$-scale Higgs triplet bosons of the Littlest Higgs or Left-Right Symmetric models at the LHC using leptonic final states would require 
several $a b^{-1}$ of integrated luminosity. This would require the capabilities of the proposed luminosity upgrades to the LHC, known as the Super LHC (SLHC). However, as is common in model building, it is possible that the existing constraints could be evaded, making these processes more useful than these conclusions pessimistically suggest.

\subsection{Summary}

In this chapter, the results of a study of Higgs triplet production via vector boson scattering at the LHC were presented. The production cross sections are highly sensitive to the triplet vacuum expectation value $\left(v^{\prime}\right)$ which is already tightly constrained by precision electroweak data. Of the three models considered, the Georgi-Machacek model can have the largest cross sections due to the weakest constraint on $v^{\prime}$. The $W^{ \pm} W^{ \pm}$channel is the most promising discovery channel due to its distinctive final state. However, it is still possible to observe a signal for the $W^{ \pm} Z$ channel for certain regions of parameter space. The observation of this channel is a smoking gun for a Higgs triplet, as this process does not occur in Two Higgs Doublet Models. Discovery of the neutral Higgs triplet state is least promising due to the large QCD and $t$-quark backgrounds in the $W^{+} W^{-}$channel and the small signal cross section in the $Z Z \rightarrow \ell^{+} \ell^{-} \ell^{+} \ell^{-}$channel. The $Z Z \rightarrow \ell^{+} \ell^{-} \nu \bar{\nu}$ channel might slightly improve the $\Phi^{0}$ discovery potential, but this has not been included in the analysis of this chapter and is left for a future study. Observing signals in some of the channels and models would require a large amount of integrated luminosity and would await the SLHC.

One of the goals of this work was to determine whether scalar triplet production via vector boson scattering at the LHC could be used to distinguish between models with Higgs triplet bosons. The current constraints on the triplet vev in the Littlest Higgs and Left-Right Symmetric models result in production cross sections that would 
require significant luminosity to be able to distinguish triplet scalars from Standard Model backgrounds, higher than the expected LHC luminosities. In these scenarios, these measurements could at best constrain the allowed parameter space. However, if the $W^{ \pm} W^{ \pm}$and $W^{ \pm} Z$ signals are observed, it would be possible to distinguish the three models by computing the ratio of the rates in those two channels. In doing so, the dependence on the triplet vev cancels out, and this ratio would be sensitive to the details each model.

The analysis of this chapter focused on the fully leptonic final states, which are easiest to distinguish from Standard Model backgrounds. The "silver-plated" semileptonic decay modes have significantly higher branching ratios and could potentially improve the statistics; the branching ratios are $B R\left(W^{ \pm} W^{ \pm} \rightarrow \ell^{ \pm} \nu \ell^{ \pm} \nu\right) \sim 5 \%$ versus $B R\left(W^{ \pm} W^{ \pm} \rightarrow \ell^{ \pm} \nu q \bar{q}\right) \sim 29 \%, B R\left(W^{ \pm} Z \rightarrow \ell^{ \pm} \nu \ell^{+} \ell^{-}\right) \sim 1.5 \%$ versus $B R\left(W^{ \pm} Z \rightarrow \ell^{ \pm} \nu q \bar{q}+q \bar{q} \ell^{+} \ell^{-}\right) \sim 20 \%$, and $B R\left(Z Z \rightarrow \ell^{+} \ell^{-} \ell^{+} \ell^{-}\right) \sim 0.44 \%$ versus $B R\left(Z Z \rightarrow \ell^{+} \ell^{-} q \bar{q}\right) \sim 9 \%$. However, much depends on the efficiency in reconstructing $W$ and $Z$ bosons in their hadronic modes. In addition, the kinematic cuts would need to be modified to take into account the hadronic final states, so this exercise is left for a future study. 


\section{Chapter 4}

\section{The Bestest Little Higgs Model}

\subsection{Introduction}

Little Higgs models $[13-17,88,89]$ offer an attractive solution to the hierarchy problem, applying collective symmetry breaking to ensure that a quadratically divergent Higgs mass is not generated at one loop. This collective symmetry breaking is implemented in the gauge, fermion, and Higgs sectors, and therefore implies the existence of partner particles for most of the Standard Model fields. However, Little Higgs models generally suffer from three major problems:

1. Although it is relatively straightforward to implement collective symmetry breaking in the gauge and fermion sectors, it is more difficult to generate the Higgs quartic coupling that is required to generate the masses of the Standard Model particles. Furthermore, the scalar sector must preserve a custodial $S U(2)$ symmetry in order to avoid large contributions to the electroweak PeskinTakeuchi $T$-parameter [75].

2. The dangerous singlet problem. Some recent Little Higgs models have been constructed that successfully generate a Higgs quartic coupling and also possess a custodial symmetry [90]. However, this required introducing additional singlet 
scalars with shift symmetries that spoiled collective symmetry breaking in a subtle way that was not recognized in these models. This generated quadratically divergent Higgs mass and singlet tadpole terms in the Higgs potential [47] and for this reason, these singlet scalars are dubbed "dangerous singlets".

3. Little Higgs models are highly constrained by precision electroweak data, which strongly limit the contribution of new physics in the gauge sector, implying that the gauge boson partners must be relatively heavy, $M_{W^{\prime}} \gtrsim 2-3 \mathrm{TeV}[91,92]$. On the other hand, avoiding fine-tuning in the top sector requires that the top partners are relatively light, $M_{T} \lesssim 1-2 \mathrm{TeV}$. However, in most Little Higgs models the top partners are heavier than the gauge boson partners:

$$
\frac{M_{T}}{M_{W^{\prime}}} \simeq \frac{M_{t}}{M_{W}} \simeq 2,
$$

which can lead to significant fine-tuning in the Higgs potential [93].

The Bestest Little Higgs model [21] was constructed to solve all three of these problems. A Higgs quartic coupling is generated without introducing any dangerous singlets to the scalar sector. Meanwhile, relatively heavy gauge boson partners allow the model to evade precision electroweak constraints, and fine-tuning is avoided in the top sector because the top partners are relatively light. The reason this model succeeds is a modular approach to model building involving two separate symmetry breaking scales, $f \sim 1 \mathrm{TeV}$ and $F>f$, at which the top partncrs and heavy gauge bosons, respectively, obtain their masses.

The details of this symmetry breaking, how it addresses the issues listed above, and the resulting particles and interactions of the Bestest Little Higgs model, are described in this chapter. In Section 4.2, the Higgs sector and Electroweak Symmetry Breaking (EWSB) are introduced. The gauge and fermion sectors are discussed in Sections 4.3 
and 4.4, respectively, and it is explicitly shown that the gauge boson partners are heavier than the top quark partners. The radiative corrections to the Higgs potential are discussed in Section 4.5 and it is shown that no fine-tuning nor dangerous singlets are introduced in this model. Additional details of the Bestest Little Higgs model are given in Appendix B. As the Bestest Little Higgs model predicts the existence of several relatively light top partners, which can be readily produced at the LHC, there is a rich phenomenology in the heavy quark sector, which is the focus of Chapter 5.

\subsection{Scalar Sector and Electroweak Symmetry Breaking}

In order to generate a Higgs quartic coupling, Little Higgs models must contain additional Pseudo Nambu-Goldstone Bosons (PNGBs) with specific quantum numbers: a one-Higgs doublet model requires an electroweak triplet, whereas a two-Higgs doublet model requires either a triplet or a singlet [47]. Since an electroweak triplet in Little Higgs models without $T$-parity [43-45] typically acquires a vev that violates custodial $S U(2)$ symmetry, the Bestest Little Higgs model is chosen to be a two-Higgs doublet model, with an additional singlet and custodial $S U(2)$ symmetry.

The simplest symmetry breaking pattern that contains the desired PNGBs, as well as a custodial symmetry, starts with a global $S O(6)_{A} \times S O(6)_{B}$ symmetry. A non-linear sigma model field, $\Sigma$, is introduced as a $6 \times 6$ matrix in a real representation of $S O(6)_{A} \times S O(6)_{B}$. When $\Sigma$ acquires a vev, given by the $6 \times 6$ unit matrix,

$$
\langle\Sigma\rangle=\mathbb{1}_{6}
$$

the global symmetry is spontaneously broken to the diagonal subgroup: $S O(6)_{A} \times$ $S O(6)_{B} \rightarrow S O(6)_{V}[21]$. Since the $S O(6)$ group has $6(6-1) / 2=15$ symmetry 
generators, according to Goldstone's theorem [31], this symmetry breaking pattern gives rise to 15 PNGBs, which can be parametrized as [21]

$$
\Sigma=e^{i \Pi / f} e^{2 i \Pi_{h} / f} e^{i \Pi / f} .
$$

The matrices $\Pi$ and $\Pi_{h}$ are $6 \times 6$ imaginary anti-symmetric matrices given by

$$
\begin{gathered}
\Pi=\left(\begin{array}{ccc}
\phi_{a} T_{L}^{a}+\eta_{a} T_{R}^{a} & 0 & 0 \\
0 & 0 & i \sigma / \sqrt{2} \\
0 & -i \sigma / \sqrt{2} & 0
\end{array}\right), \\
\Pi_{h}=\frac{i}{\sqrt{2}}\left(\begin{array}{ccc}
0_{4 \times 4} & h_{1} & h_{2} \\
-h_{1}^{T} & 0 & 0 \\
-h_{2}^{T} & 0 & 0
\end{array}\right)
\end{gathered}
$$

where

$$
h_{i}^{T}=\left(h_{i 1}, h_{i 2}, h_{i 3}, h_{i 4}\right)
$$

are 4's of $S O(4)$, which can alternatively be written as complex Higgs doublets in the following way:

$$
H_{i}=\frac{1}{\sqrt{2}}\left(\begin{array}{c}
h_{i 3}+i h_{i 4} \\
h_{i 1}-i h_{i 2}
\end{array}\right) \quad(i=1,2) .
$$

However, in the discussion of the Bestest Little Higgs model in this thesis, the real vector notation of Equation 4.6 is used, rather than the complex doublet notation of Equation 4.7. $\sigma$ is a real singlet field that is necessary for generating a collective 
quartic coupling. This parametrization of the scalar fields is useful because the Higgs fields of Equation 4.5 are separated from the other PNGBs of Equation 4.4 in order to avoid mixing between these states after EWSB.

The $4 \times 4$ matrices $T_{L, R}^{a}(a=1,2,3)$ are the generators of $S U(2)_{L}$ and $S U(2)_{R}$ contained in $S O(4)$ and are given in Appendix B by Equations B.1-B.3. It should be noted that the upper-left $4 \times 4$ block of $S O(6)_{A}$ is decomposed into an $S U(2)_{L A} \times S U(2)_{R A}$ symmetry and that the upper-left $4 \times 4$ block of $S O(6)_{B}$ is decomposed into an $S U(2)_{L B} \times S U(2)_{R B}$ symmetry. Therefore, the diagonal combination of $S U(2)_{L A}$ and $S U(2)_{L B}$ is weakly gauged and identified as the Standard Model $S U(2)_{L}$ subgroup:

$$
T_{L}^{a}=T_{L A}^{a}+T_{L B}^{a},(a=1,2,3)
$$

The diagonal combination of the third component of $S U(2)_{R}$ is also gauged and identified as the Standard Model hypercharge:

$$
Y=T_{R}^{3}=T_{R A}^{3}+T_{R B}^{3}
$$

The field $\phi^{a}(a=1,2,3)$ is an electroweak triplet with zero hypercharge. The field $\eta^{a}(a=1,2,3)$ is an $S U(2)_{R}$ triplet, meaning that $\eta_{1}$ and $\eta_{2}$ form a complex singlet with hypercharge and $\eta_{3}$ is a real singlet.

In order to generate a Higgs quartic coupling, collective symmetry breaking requires two operators, each of which explicitly breaks enough symmetries under which the Higgs transforms non-linearly, but neither by itself allows the Higgs to get a 
potential. To do this, it is useful to define the projection operators

$$
\begin{aligned}
& P_{5}=\operatorname{diag}(0,0,0,0,1,0) \\
& P_{6}=\operatorname{diag}(0,0,0,0,0,1)
\end{aligned}
$$

then write the quartic potential as [21]

$$
\begin{aligned}
V_{\text {quartic }} & =\frac{1}{4} \lambda_{56} f^{4} \operatorname{Tr}\left(P_{5} \Sigma P_{6} \Sigma^{T}\right)+\frac{1}{4} \lambda_{65} f^{4} \operatorname{Tr}\left(P_{6} \Sigma P_{5} \Sigma^{T}\right) \\
& =\frac{1}{4} \lambda_{56} f^{4}\left(\Sigma_{56}\right)^{2}+\frac{1}{4} \lambda_{65} f^{4}\left(\Sigma_{65}\right)^{2} .
\end{aligned}
$$

The first term in Equation 4.12 explicitly breaks $S O(6)_{A} \times S O(6)_{B} \rightarrow S O(5)_{A 5} \times$ $S O(5)_{B 6}$, where the $S O(5)_{A 5}$ transformation does not act on the fifth row or column, and therefore protects $h_{1}$ from getting a potential, while the $S O(5)_{B 6}$ transformation does not act on the sixth row or column, and therefore protects $h_{2}$. Similarly, the second term in Equation 4.12 explicitly breaks $S O(6)_{A} \times S O(6)_{B} \rightarrow S O(5)_{A 6} \times$ $S O(5)_{B 5}$.

Expanding Equation 4.3 as a power series in $1 / f$ gives

$$
\Sigma=\mathbb{1}_{6}+2 i \frac{\left(\Pi+\Pi_{h}\right)}{f}-2 \frac{\left(\Pi+\Pi_{h}\right)^{2}}{f^{2}}+\ldots
$$

with $\Pi$ and $\Pi_{h}$ defined in Equations 4.4 and 4.5, respectively. Substitution of Equation 4.13 into Equation 4.12 gives

$$
V_{\text {quartic }}=\frac{\lambda_{56}}{2}\left(f \sigma+\frac{1}{\sqrt{2}} h_{1}^{T} h_{2}+\ldots\right)^{2}+\frac{\lambda_{65}}{2}\left(f \sigma-\frac{1}{\sqrt{2}} h_{1}^{T} h_{2}+\ldots\right)^{2}
$$

which is similar to the form of Equation 2.36. This generates a mass term for the 
singlet, $\sigma$, of the form

$$
\mathcal{L}_{\sigma \text { mass }}=\frac{1}{2} m_{\sigma} \sigma^{2}, \quad \text { where } \quad m_{\sigma}^{2}=\left(\lambda_{56}+\lambda_{65}\right) f^{2}
$$

There are no mass terms for the Higgs doublet fields, as there are no $h_{1}^{T} h_{1}$ or $h_{2}^{T} h_{2}$ terms in Equation 4.14.

While each of the two operators in Equation 4.14 individually appear to generate a Higgs quartic, this quartic can be eliminated by a field redefinition

$$
\sigma \rightarrow \sigma \mp \frac{h_{1}^{T} h_{2}}{\sqrt{2} f}
$$

where the upper and lower signs of this transformation correspond to the first and second operators of Equation 4.14, respectively. However, in the presence of both terms in Equation 4.14, a Higgs quartic can be generated collectively by integrating out the $\sigma$ field, which will be seen below.

The full scalar potential can be written as

$$
V=V_{\text {quartic }}+V_{\text {sym break }}+V_{B_{\mu}}
$$

where $V_{\text {quartic }}$ is the quartic potential defined in Equation 4.12 (or alternatively, Equation 4.14). In the absence of gauge interactions, not all the scalars obtain masses. To generate them, the following small symmetry breaking term is added [21]:

$$
V_{\text {sym break }}=-\frac{f^{2}}{4} m_{4}^{2} \operatorname{Tr}\left(\Delta^{\dagger} M_{26} \Sigma M_{26}^{\dagger}+\Delta M_{26} \Sigma^{\dagger} M_{26}^{\dagger}\right)-\frac{f^{2}}{4}\left(m_{5}^{2} \Sigma_{55}+m_{6}^{2} \Sigma_{66}\right)
$$

where $\Delta$ is a field that breaks a global $S U(2)_{C} \times S U(2)_{D}$ symmetry to the diagonal at some higher scale $F>f$. The motivation for introducing this field will be explained when introducing the gauge sector in Section 4.3 . It can be parametrized as a $2 \times 2$ 
matrix, given by

$$
\Delta=e^{2 i \Pi_{d} / F}, \quad \Pi_{d}=\chi_{a} \frac{\tau_{a}}{2} \quad(a=1,2,3)
$$

where the triplet $\chi_{a}$ mixes with the triplet $\phi_{a}$, as shown in Section B.2 of Appendix B. $M_{26}$, which is a $2 \times 6$ matrix that contracts the $S U(2)$ indices of $\Delta$ with the $S O(6)$ indices of $\Sigma$, is given by

$$
M_{26}=\frac{1}{\sqrt{2}}\left(\begin{array}{cccccc}
0 & 0 & 1 & i & 0 & 0 \\
1 & -i & 0 & 0 & 0 & 0
\end{array}\right)
$$

This matrix is useful for converting the Higgs fields from the vector notation of Equation 4.6 to the doublet notation of Equation 4.7. Expanding $\Delta$ as a power series in $1 / F$,

$$
\Delta=\mathbb{1}_{2}+2 i \frac{\Pi_{d}}{F}-2 \frac{\Pi_{d}^{2}}{F^{2}}+\ldots
$$

and substituting this into Equation 4.18, retaining only the mass terms, gives

$$
V_{\text {sym break }}=\frac{1}{2} m_{\phi}^{2} \phi_{a}^{2}+\frac{1}{2} m_{\eta}^{2} \eta_{a}^{2}+\frac{1}{2} m_{1}^{2} h_{1}^{T} h_{1}+\frac{1}{2} m_{2}^{2} h_{2}^{T} h_{2}+\frac{1}{4}\left(m_{5}^{2}+m_{6}^{2}\right) \sigma^{2}
$$

It is evident that the $h_{1}, h_{2}, \phi_{a}$ and $\eta_{a}$ fields have all obtained masses given by

$$
\begin{aligned}
m_{\dot{\phi}}^{2} & =m_{\eta}^{2}=m_{4}^{2} \\
m_{1}^{2} & =\frac{1}{2}\left(m_{4}^{2}+m_{5}^{2}\right) \\
m_{2}^{2} & =\frac{1}{2}\left(m_{4}^{2}+m_{6}^{2}\right)
\end{aligned}
$$

It should be noted that these mass terms do not include the larger contributions from radiative corrections, which will be examined in Section 4.5. It is also evident that the singlet, $\sigma$, has acquired a correction to its mass, given by $\frac{1}{2}\left(m_{5}^{2}+m_{6}^{2}\right)$. However, since 
$m_{5}$ and $m_{6}$ can be chosen to be smaller than the electroweak scale, this correction is neglected compared to the mass of order $f \sim 1 \mathrm{TeV}$ given by Equation 4.15.

In order to destabilise the origin and trigger EWSB, the following terms are also added to the scalar potential [21]:

$$
\begin{aligned}
V_{B_{\mu}} & =m_{56}^{2} f^{2} \Sigma_{56}+m_{65}^{2} f^{2} \Sigma_{65} \\
& =\sqrt{2}\left(m_{65}^{2}-m_{56}^{2}\right) f \sigma-\left(m_{56}^{2}+m_{65}^{2}\right) h_{1}^{T} h_{2}+\ldots
\end{aligned}
$$

Although the first term in the second line of Equation 4.26 appears to have generated a singlet tadpole, this term is not quadratically divergent. Furthermore, since $m_{56}$ and $m_{65}$ can be naturally at the weak scale without introducing fine-tuning, this singlet tadpole is not dangerous. Since $\sigma$ is the heaviest scalar, the full scalar potential may be analyzed in the limit $f \gg v$ by integrating it out. This can be seen by minimizing the potential of Equation 4.17 with respect to $\sigma$ and substituting the resulting solution for $\sigma$ back into Equation 4.17, similar to the procedure shown in Equation 2.40. This results in a potential for the Higgs doublet fields, given by

$$
V_{\text {Higgs }}=\frac{1}{2} m_{1}^{2} h_{1}^{T} h_{1}+\frac{1}{2} m_{2}^{2} h_{2}^{T} h_{2}-B_{\mu} h_{1}^{T} h_{2}+\frac{\lambda_{0}}{2}\left(h_{1}^{T} h_{2}\right)^{2}
$$

where

$$
B_{\mu}=2 \frac{\lambda_{56} m_{65}^{2}+\lambda_{65} m_{56}^{2}}{\lambda_{56}+\lambda_{65}}
$$

For the potential to have a minimum, one must have $m_{1}, m_{2}>0$, while electroweak symmetry breaking requires $B_{\mu}>m_{1} m_{2}$.

It is evident from Equation 4.27 that a Higgs quartic coupling has been successfully generated, given by

$$
\lambda_{0}=2 \frac{\lambda_{56} \lambda_{65}}{\lambda_{56}+\lambda_{65}}
$$


which vanishes if either $\lambda_{56}=0$ or $\lambda_{65}=0$ (if both $\lambda_{56}$ and $\lambda_{65}$ are zero, the potential of Equation 4.12 vanishes altogether and a quartic cannot be generated). This is the essence of collective symmetry breaking and reaffirms that both operators of Equation 4.12 are indeed required to generate a Higgs quartic. Mass terms and a $B_{\mu}$-like mass term for the Higgs doublet fields have also been generated. Since the scalar fields have been arranged in such a way that the Higgs doublet fields do not mix with the other scalars, it is not necessary to consider terms involving the triplet fields, $\phi_{a}, \eta_{a}$ and $\chi_{a}$, when determining the mass eigenstates - that is, the physical particles that interact with each other - for the Higgs doublet fields.

Electroweak Symmetry Breaking in the Bestest Little Higgs model occurs when the Higgs doublets acquire vevs given by

$$
\begin{aligned}
& \left\langle h_{1}\right\rangle^{T}=\left(v_{1}, 0,0,0\right) \\
& \left\langle h_{2}\right\rangle^{T}=\left(v_{2}, 0,0,0\right) .
\end{aligned}
$$

Requiring that these vevs minimize the Higgs potential of Equation 4.27 , leads to the relations

$$
\begin{aligned}
& v_{1}^{2}=\frac{1}{\lambda_{0}} \frac{m_{2}}{m_{1}}\left(B_{\mu}-m_{1} m_{2}\right) \\
& v_{2}^{2}=\frac{1}{\lambda_{0}} \frac{m_{1}}{m_{2}}\left(B_{\mu}-m_{1} m_{2}\right)
\end{aligned}
$$

These parameters can be more conveniently expressed in terms of the parameters $v$ and $\tan \beta$ as follows

$$
\begin{gathered}
v^{2} \equiv v_{1}^{2}+v_{2}^{2}=\frac{1}{\lambda_{0}}\left(\frac{m_{1}^{2}+m_{2}^{2}}{m_{1} m_{2}}\right)\left(B_{\mu}-m_{1} m_{2}\right) \simeq(246 \mathrm{GeV})^{2} \\
\tan \beta \equiv \frac{v_{1}}{v_{2}}=\frac{m_{2}}{m_{1}}
\end{gathered}
$$


By shifting the Higgs doublet fields by their vevs

$$
h_{i 1} \rightarrow h_{i 1}+v_{i} \quad(i=1,2)
$$

in the Higgs potential of Equation 4.27, the Higgs doublet mass terms after EWSB can be determined. By diagonalizing the Higgs doublet mass matrices, the physical Higgs doublet mass eigenstates are found to be mixtures of the gauge eigenstates, $h_{i j}(i=1,2, j=1,2,3,4)$, that appear in the Higgs potential of Equation 4.27. The details of this diagonalization and the form of the Higgs doublet mass eigenstates are given in Section B.2 of Appendix B.

The resulting physical Higgs doublet states include the three massless Goldstone bosons, $G^{ \pm}$and $G^{0}$, which are eaten to give the Standard Model $W^{ \pm}$and $Z$ bosons their masses, as well as the massive states $h^{0}, A^{0}, H^{ \pm}$and $H^{0}$, with masses given by

$$
\begin{aligned}
M_{G^{0}} & =M_{G^{ \pm}}=0 \\
M_{A^{0}}^{2} & =M_{H^{ \pm}}^{2}=m_{1}^{2}+m_{2}^{2}=\frac{2 B_{\mu}}{\sin 2 \beta}-\lambda_{0} v^{2}, \\
M_{h^{0}, H^{0}}^{2} & =\frac{B_{\mu}}{\sin 2 \beta} \mp \sqrt{\frac{B_{\mu}^{2}}{\sin ^{2} 2 \beta}-2 \lambda_{0} B_{\mu} v^{2} \sin 2 \beta+\lambda_{0}^{2} v^{4} \sin ^{2} 2 \beta .}
\end{aligned}
$$

The massive states follow a hierarchy

$$
m_{h^{0}}^{2}<m_{A^{0}}^{2}=m_{H^{ \pm}}^{2}<m_{H^{0}}^{2}
$$

where the lightest $h^{0}$ state is identified as the Standard Model Higgs boson.

The scalar sector also includes the triplets $\phi^{a}, \eta^{a}$ and $\chi^{a}(a=1,2,3)$, the mass eigenstates of which are also listed in Section B.2 of Appendix B. 


\subsection{Gauge Sector}

As stated in the introduction to this chapter, precision electroweak measurements strongly limit the contribution of new physics in the gauge sector, implying that the gauge boson partners must be relatively heavy, $M_{W^{\prime}} \gtrsim 2-3 \mathrm{TeV}$. On the other hand, the absence of fine-tuning in the top sector requires that the top partners are relatively light, $M_{T} \lesssim 1-2 \mathrm{TeV}$. However, most Little Higgs models predict the opposite mass hierarchy in which the top partners are heavier than the gauge boson partners. In order to solve this problem, the Bestest Little Higgs model uses a modular approach for building a collective gauge coupling, in which the dynamics that generate the quartic and fermion interactions occur at the scale $f$, while the dynamics that cut off the gauge boson quadratic divergences occur at a higher scale $F>f$. The reason for this is that $F$ can be made large enough to evade precision electroweak constraints in the gauge sector, without causing any fine-tuning in the quartic or fermion sectors.

More explicitly, the model employs two independent non-linear sigma models, $\Sigma$ and $\Delta$, shown schematically in Figure 4.1. The symmetry breaking occurs in two stages [21]:

1. $\Sigma$ breaks a global $S O(6)_{A} \times S O(6)_{B}$ symmetry down to the diagonal at a scale $f$.

2. $\Delta$ breaks a global $S U(2)_{C} \times S U(2)_{D}$ symmetry down to the diagonal at a scale $F>f$

Connecting these two non-linear sigma models is accomplished by gauging the $S U(2)_{L A}$ symmetry in $S O(6)_{A}$ and the $S U(2)_{C}$ symmetry with the same $S U(2)_{A}$ gauge bosons. Similarly, the $S U(2)_{L B}$ symmetry in $S O(6)_{B}$ and the $S U(2)_{D}$ symmetry are gauged with the same $S U(2)_{B}$ gauge bosons. $S U(2)_{A, B}$ are subgroups of $S O(6)_{A, B}$, whose diagonal subgroup is the Standard Model $S U(2)_{L}$. 
Global: $\quad S O(6)_{A}$

$S O(6)_{B}$

$S U(2)_{C}$

$S U(2)_{D}$
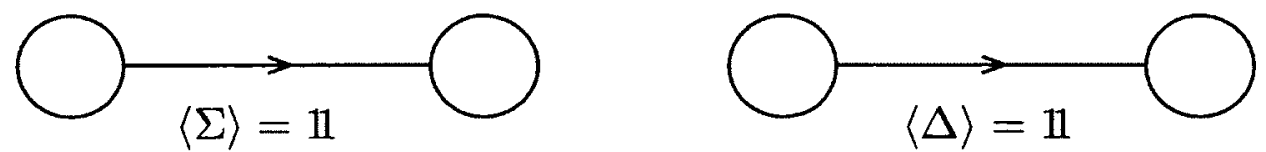

Gauged: $\quad S U(2)_{A} \quad U(1)_{Y} \quad S U(2)_{B}$

$S U(2)_{A}$

$S U(2)_{B}$

Figure 4.1: The modular structure of the symmetry breaking in the Bestest Little Higgs model. $\Sigma$ breaks a global $S O(6)_{A} \times S O(6)_{B}$ symmetry down to the diagonal at a scale $f$, while $\Delta$ breaks a global $S U(2)_{C} \times S U(2)_{D}$ symmetry down to the diagonal at a scale $F>f$. The $S U(2)_{L A}$ symmetry in $S O(6)_{A}$ and the $S U(2)_{C}$ symmetry are gauged with the same $S U(2)_{A}$ gauge bosons. Similarly, the $S U(2)_{L B}$ symmetry in $S O(6)_{B}$ and the $S U(2)_{D}$ symmetry are gauged with the same $S U(2)_{B}$ gauge bosons. $S U(2)_{A, B}$ are subgroups of $S O(6)_{A, B}$, whose diagonal subgroup is the Standard Model $S U(2)_{L}$.

The gauge kinetic terms of the electroweak Lagrangian in the Bestest Little Higgs model are [21]

$$
\mathcal{L}_{\text {gauge }}=\frac{f^{2}}{8} \operatorname{Tr}\left(D_{\mu} \Sigma^{\dagger} D^{\mu} \Sigma\right)+\frac{F^{2}}{4} \operatorname{Tr}\left(D_{\mu} \Delta^{\dagger} D^{\mu} \Delta\right)
$$

where the covariant derivatives are given by

$$
\begin{aligned}
D_{\mu} \Sigma & =\partial_{\mu} \Sigma+i g_{A} A_{1 \mu}^{a} T^{a} \Sigma-i g_{B} \Sigma A_{2 \mu}^{a} T^{a}+i g^{\prime}\left[B_{\mu}^{3} T^{\prime 3}, \Sigma\right] \\
D_{\mu} \Delta & =\partial_{\mu} \Delta+i g_{A} A_{1 \mu}^{a} \frac{\tau^{a}}{2} \Delta-i g_{B} \Delta A_{2 \mu}^{a} \frac{\tau^{a}}{2}
\end{aligned}
$$

with the $S O(6)$ generators $T^{a}$ and $T^{\prime a}$ defined in Appendix B. $g_{A}$ and $A_{1 \mu}^{a}(a=$ $1,2,3)$ are the gauge coupling and field associated with the $S U(2)_{L A}$ gauge bosons, $g_{B}$ and $A_{2 \mu}^{a}(a=1,2,3)$ are the gauge coupling and field associated with the $S U(2)_{L B}$ gauge bosons, while $g^{\prime}$ and $B_{\mu}^{3}$ are the hypercharge coupling and field respectively. Hypercharge was chosen to be gauged as the diagonal combination of $T_{R}^{3}$ in the $\Sigma$ 
sector, leaving the $\Delta$ sector unchanged [21].

The gauge boson mass eigenstates were determined by first shifting the scalar fields by their vevs, $h_{i 1} \rightarrow h_{i 1}+v_{i}(i=1,2)$, in the Lagrangian of Equation 4.41. The resulting gauge boson mass matrices were then expanded as a power series in $\frac{v^{2}}{f}$ using Mathematica version 7.0 [94] and diagonalized up to $\mathcal{O}\left(\frac{v^{4}}{f^{2}}\right)$ using perturbation theory. The details of this diagonalization precedure are given in Section B.3 of Appendix B.

The resulting physical gauge bosons include the Standard Model gauge bosons, $\gamma$, $W^{ \pm}$and $Z$, as well as the heavy gauge boson partners, $W^{ \pm}$and $Z^{\prime}$. Their masses, up to $\mathcal{O}\left(\frac{v^{4}}{f^{2}+F^{2}}\right)$, were calculated to be

$$
\begin{aligned}
M_{\gamma}^{2}= & 0 \\
M_{Z}^{2}= & \frac{1}{4}\left(g^{2}+g^{\prime 2}\right) v^{2}-\left(g^{2}+g^{2}\right)\left(2+\frac{3 f^{2}}{f^{2}+F^{2}}\left(s_{g}^{2}-c_{g}^{2}\right)^{2}\right) \frac{v^{4}}{48 f^{2}} \\
M_{W}^{2}= & \frac{1}{4} g^{2} v^{2}-g^{2}\left(2+\frac{3 f^{2}}{f^{2}+F^{2}}\left(s_{g}^{2}-c_{g}^{2}\right)^{2}\right) \frac{v^{4}}{48 f^{2}} \\
M_{Z^{\prime}}^{2}= & \frac{1}{4}\left(g_{A}^{2}+g_{B}^{2}\right)\left(f^{2}+F^{2}\right)-\frac{1}{4} g^{2} v^{2} \\
& +\left(2 g^{2}+\frac{3 f^{2}}{f^{2}+F^{2}}\left(g^{2}+g^{2}\right)\left(s_{g}^{2}-c_{g}^{2}\right)^{2}\right) \frac{v^{4}}{48 f^{2}} \\
M_{W^{\prime}}^{2}= & \frac{1}{4}\left(g_{A}^{2}+g_{B}^{2}\right)\left(f^{2}+F^{2}\right)-M_{W}^{2}
\end{aligned}
$$

where the unbroken $S U(2)_{L}$ coupling is given by

$$
g=\frac{g_{A} g_{B}}{\sqrt{g_{A}^{2}+g_{B}^{2}}}
$$

and the mixing angles are given by

$$
s_{g}=\sin \theta_{g}=\frac{g_{A}}{\sqrt{g_{A}^{2}+g_{B}^{2}}}, \quad c_{g}=\cos \theta_{g}=\frac{g_{B}}{\sqrt{g_{A}^{2}+g_{B}^{2}}}
$$


The weak mixing angle is defined as

$s_{W}=\sin \theta_{W}=\frac{g^{\prime}}{\sqrt{g^{2}+g^{\prime 2}}}, \quad c_{W}=\cos \theta_{W}=\frac{g}{\sqrt{g^{2}+g^{\prime 2}}}, \quad x_{W}=\frac{1}{2 c_{W}} s_{g} c_{g}\left(s_{g}^{2}-c_{g}^{2}\right)$

It is evident that the heavy gauge bosons, $W^{\prime \pm}$ and $Z^{\prime}$, have acquired masses at the scale $F>f$, as desired.

\subsection{Fermion Sector}

To build the Yukawa interactions, the fermions must transform under either $S O(6)_{A}$ or $S O(6)_{B}$. As the fundamental representation (6) of $S O(6)$ contains two $S U(2)_{L}$ doublets and two singlets, the fermions transforming under the $S O(6)_{A}$ fundamental representation are

$$
Q^{T}=\left(\frac{1}{\sqrt{2}}\left(-Q_{a 1}-Q_{b 2}\right), \frac{i}{\sqrt{2}}\left(Q_{a 1}-Q_{b 2}\right), \frac{1}{\sqrt{2}}\left(Q_{a 2}-Q_{b 1}\right), \frac{i}{\sqrt{2}}\left(Q_{a 2}+Q_{b 1}\right), Q_{5}, Q_{6}\right)
$$

where $Q_{a}=\left(Q_{a 1}, Q_{a 2}\right)$ and $Q_{b}=\left(Q_{b 1}, Q_{b 2}\right)$ are $S U(2)_{L}$ doublets with hypercharge $-\frac{1}{2}$ and $+\frac{1}{2}$, respectively, and $Q_{5}$ and $Q_{6}$ are singlets under $S U(2)_{L} \times S U(2)_{R} \equiv S O(4)$. In order to identify $Q_{a}$ as the Standard Model quark doublet with hypercharge $\frac{1}{6}$ (as in Table 2.2), the definition of Equation 4.9 for the hypercharge operator acting on the fermions must be modified. This is accomplished by gauging a linear combination of $T_{R}^{3}$ and a global symmetry of the fermion sector $U(1)_{X}$. The hypercharge operator in the fermion sector is therefore given by

$$
Y=T_{R}^{3}+T_{X}=Q_{E M}-T_{L}^{3}
$$


Table 4.1: Quantum numbers for the fermions in the Bestest Little Higgs model.

\begin{tabular}{|c|c|c|c|c|c|}
\hline Fermion & $T_{X}$ & $T_{R}^{3}$ & $T_{L}^{3}$ & $Y$ & $Q_{E M}$ \\
\hline$Q_{a 1}$ & $+\frac{2}{3}$ & $-\frac{1}{2}$ & $+\frac{1}{2}$ & $+\frac{1}{6}$ & $+\frac{2}{3}$ \\
\hline$Q_{a 2}$ & $+\frac{2}{3}$ & $-\frac{1}{2}$ & $-\frac{1}{2}$ & $+\frac{1}{6}$ & $-\frac{1}{3}$ \\
\hline$Q_{b 1}$ & $+\frac{2}{3}$ & $+\frac{1}{2}$ & $+\frac{1}{2}$ & $+\frac{7}{6}$ & $+\frac{5}{3}$ \\
\hline$Q_{b 2}$ & $+\frac{2}{3}$ & $+\frac{1}{2}$ & $-\frac{1}{2}$ & $+\frac{7}{6}$ & $+\frac{2}{3}$ \\
\hline$Q_{5}$ & $+\frac{2}{3}$ & 0 & 0 & $+\frac{2}{3}$ & $+\frac{2}{3}$ \\
\hline$Q_{6}$ & $+\frac{2}{3}$ & 0 & 0 & $+\frac{2}{3}$ & $+\frac{2}{3}$ \\
\hline$Q_{a 1}^{\prime}$ & $+\frac{2}{3}$ & $-\frac{1}{2}$ & $+\frac{1}{2}$ & $+\frac{1}{6}$ & $+\frac{2}{3}$ \\
\hline$Q_{a 2}^{\prime}$ & $+\frac{2}{3}$ & $-\frac{1}{2}$ & $-\frac{1}{2}$ & $+\frac{1}{6}$ & $-\frac{1}{3}$ \\
\hline$U_{a 1}^{c}$ & $-\frac{2}{3}$ & $+\frac{1}{2}$ & $+\frac{1}{2}$ & $-\frac{1}{6}$ & $+\frac{1}{3}$ \\
\hline$U_{a 2}^{c}$ & $-\frac{2}{3}$ & $+\frac{1}{2}$ & $-\frac{1}{2}$ & $-\frac{1}{6}$ & $-\frac{2}{3}$ \\
\hline$U_{b 1}^{c}$ & $-\frac{2}{3}$ & $-\frac{1}{2}$ & $+\frac{1}{2}$ & $-\frac{7}{6}$ & $-\frac{2}{3}$ \\
\hline$U_{b 2}^{c}$ & $-\frac{2}{3}$ & $-\frac{1}{2}$ & $-\frac{1}{2}$ & $-\frac{7}{6}$ & $-\frac{5}{3}$ \\
\hline$U_{5}^{c}$ & $-\frac{2}{3}$ & 0 & 0 & $-\frac{2}{3}$ & $-\frac{2}{3}$ \\
\hline$U_{6}^{c}$ & $-\frac{2}{3}$ & 0 & 0 & $-\frac{2}{3}$ & $-\frac{2}{3}$ \\
\hline$U_{5}^{c c}$ & $-\frac{2}{3}$ & 0 & 0 & $-\frac{2}{3}$ & $-\frac{2}{3}$ \\
\hline$b^{c}$ & $+\frac{1}{3}$ & 0 & 0 & $+\frac{1}{3}$ & $+\frac{1}{3}$ \\
\hline
\end{tabular}

where the second relation follows from Equation 2.8. The $U(1)_{X}$ charges of the fermions are chosen to obtain the correct hypercharges, as shown in Table 4.1.

The fermions transforming under the $S O(6)_{B}$ fundamental representation are

$$
\left(U^{c}\right)^{T}=\left(\frac{1}{\sqrt{2}}\left(-U_{b 1}^{c}-U_{a 2}^{c}\right), \frac{i}{\sqrt{2}}\left(U_{b 1}^{c}-U_{a 2}^{c}\right), \frac{1}{\sqrt{2}}\left(U_{b 2}^{c}-U_{a 1}^{c}\right), \frac{i}{\sqrt{2}}\left(U_{b 2}^{c}+U_{a 1}^{c}\right), U_{5}^{c}, U_{6}^{c}\right)
$$


where the two $S U(2)_{L}$ doublets are given by

$$
\begin{aligned}
& U_{a}^{c}=\left(U_{a 2}^{c},-U_{a 1}^{c}\right)=\left(U_{a 1}^{c}, U_{a 2}^{c}\right)\left(-i \tau^{2}\right) \\
& U_{b}^{c}=\left(-U_{b 2}^{c}, U_{b 1}^{c}\right)=\left(U_{b 1}^{c}, U_{b 2}^{c}\right)\left(+i \tau^{2}\right)
\end{aligned}
$$

along with the singlets $U_{5}^{c}$ and $U_{6}^{c}$.

The largest coupling in the fermion sector is the top Yukawa coupling, which can be generated by the following Lagrangian [21]:

$$
\mathcal{L}_{t}=y_{1} f Q^{T} S \Sigma S U^{c}+y_{2} f Q_{a}^{\prime T} \Sigma U^{c}+y_{3} f Q^{T} \Sigma U_{5}^{\prime c}+y_{b} f q_{3}^{T}\left(-2 i T_{2}^{\prime} \Sigma\right) U_{b}^{c}+\text { h.c. }
$$

where

$$
\begin{aligned}
Q_{a}^{\prime T} & =\frac{1}{\sqrt{2}}\left(-Q_{a 1}^{\prime}, i Q_{a 1}^{\prime}, Q_{a 2}^{\prime}, i Q_{a 2}^{\prime}, 0,0\right) \\
U_{5}^{\prime c T} & =\left(0,0,0,0, U_{5}^{\prime c}, 0\right)
\end{aligned}
$$

are a doublet $\left(2\right.$ of $\left.S U(2)_{A}\right)$ and a singlet $\left(1\right.$ of $\left.S U(2)_{A, B}\right)$, respectively, which can be considered as incomplete multiplets of $S O(6)_{A}$ and $S O(6)_{B}$. The $S O(6)$ matrix, $S$, is given by

$$
S=\operatorname{diag}(1,1,1,1,-1,-1)
$$

For simplicity, the Yukawa couplings are assumed to be real: $y_{1}, y_{2}, y_{3} \in \mathbb{R}$.

In the first term of Equation 4.58, the $S$ matrices explicitly break the $S O(6)_{A}$ and $S O(6)_{B}$ symmetries. They can be removed by absorbing them into the definitions of the fermion fields, in which case the first term would preserve $S O(6)_{A} \times S O(6)_{B}$, but this would cause the $S$ matrices to instead appear in the second and third terms. The second term of Equation 4.58 preserves $S O(6)_{B}$, but breaks $S O(6)_{A}$, whereas the third term of Equation 4.58 preserves $S O(6)_{A}$, but breaks $S O(6)_{B}$. Therefore, each of 
the first three terms of Equation 4.58 are required to collectively break the symmetries protecting the Higgs so that a top Yukawa coupling and radiative corrections to the Higgs potential from fermion loops can be generated.

The last term of Equation 4.58, where the right-handed $b$-quark is contained in

$$
\left(U_{b}^{c}\right)^{T}=\left(0,0,0,0, b^{c}, 0\right)
$$

and $q_{3}$ is the left-handed third generation quark doublet of the Standard Model, is included to obtain the correct Yukawa coupling for the Standard Model $b$-quark, which, although smaller than the top quark Yukawa coupling, is non-negligible. It has been coupled to the charge conjugate Higgs (the charge conjugate of $\Sigma$ is $-2 i T_{2}^{\prime} \Sigma$ in $S O(4)$ notation).

The light quarks, $u, d, c, s$, and the leptons, $e, \mu, \tau$, can be included in a similar manner. However, their Yukawa couplings explicitly break the global $S O(6)_{A}$ and $S O(6)_{B}$ symmetries without requiring collective symmetry breaking, and gauge invariant mass terms for the light fermions are included without introducing any fine-tuning to the theory [21]. Cancelling any quadratic divergences due to the light fermions does not require additional fermion partners, as these divergences are naturally suppressed due to much smaller Yukawa couplings.

Replacing $\Sigma \rightarrow\langle\Sigma\rangle=\mathbb{1}_{6}$, the mass terms of Equation 4.58 prior to EWSB can be written as

$$
\mathcal{L}_{t \text { mass }}=m_{T_{a}} T^{a} U_{a}^{c}+m_{T_{b}} T^{b} U_{b}^{c}+m_{T_{5}} Q_{5} T_{5}^{c}+m_{T_{6}} Q_{6} T_{6}^{c}+\text { h.c. }
$$


where the mass eigenstates prior to EWSB are

$$
\begin{aligned}
T_{a} & =\frac{1}{\sqrt{y_{1}^{2}+y_{2}^{2}}}\left(y_{1} Q_{a}+y_{2} Q_{a}^{\prime}\right) \\
T_{b} & =Q_{b} \\
T_{5}^{c} & =\frac{1}{\sqrt{y_{1}^{2}+y_{3}^{2}}}\left(y_{1} U_{5}^{c}+y_{3} U_{5}^{\prime c}\right), \\
T_{6}^{c} & =U_{6}^{c},
\end{aligned}
$$

with masses

$$
\begin{aligned}
& m_{T_{a}}=\sqrt{y_{1}^{2}+y_{2}^{2}} f, \\
& m_{T_{b}}=y_{1} f, \\
& m_{T_{5}}=\sqrt{y_{1}^{2}+y_{3}^{2}} f, \\
& m_{T_{6}}=y_{1} f .
\end{aligned}
$$

The states orthogonal to $T_{a}$ and $T_{5}^{c}$, given by

$$
\begin{aligned}
q_{3} & =\frac{1}{\sqrt{y_{1}^{2}+y_{2}^{2}}}\left(y_{2} Q_{a}-y_{1} Q_{a}^{\prime}\right), \\
u_{3}^{c} & =\frac{1}{\sqrt{y_{1}^{2}+y_{3}^{2}}}\left(y_{3} U_{5}^{c}-y_{1} U_{5}^{\prime c}\right),
\end{aligned}
$$

are the Standard Model third generation left-handed quark doublet and right-handed up-type singlet, respectively, which remain massless prior to EWSB.

The fermion mass eigenstates after EWSB were computed in the same manner as the gauge boson mass eigenstates. The scalar fields were first shifted by their vevs, $h_{i 1} \rightarrow h_{i 1}+v_{i}(i=1,2)$, and inserted into the Lagrangian of Equation 4.58. The resulting mass terms were expanded as a power series in $\frac{v^{2}}{f^{2}}$ using Mathematica version $7.0[94]$ and the mass matrices were diagonalized using perturbation theory. 
The details of this procedure are described in Section B.4 of Appendix B.

The fermion mass terms after diagonalization are

$$
\begin{aligned}
\mathcal{L}_{t \text { mass }}= & M_{b} b^{c} b+M_{t} t^{c} t+M_{T_{b}^{(5 / 3)}}\left(T_{b}^{(5 / 3)}\right)^{c} T_{b}^{(5 / 3)}+M_{T_{b}^{(2 / 3)}}\left(T_{b}^{(2 / 3)}\right)^{c} T_{b}^{(2 / 3)} \\
& +M_{T_{6}} T_{6}^{c} T_{6}+M_{T_{5}} T_{5}^{c} T_{5}+M_{T_{a}^{d}}\left(T_{a}^{d}\right)^{c} T_{a}^{d}+M_{T_{a}^{u}}\left(T_{a}^{u}\right)^{c} T_{a}^{u}
\end{aligned}
$$

The fermion mass eigenstates include one charge $5 / 3$ heavy quark:

$$
T_{b}^{(5 / 3)}=T_{b 1}, \quad\left(T_{b}^{(5 / 3)}\right)^{c}=-U_{b 2}^{c}
$$

two charge $-1 / 3$ quarks (one of which is the Standard Model $b$-quark) that do not mix with one another:

$$
\begin{array}{rlrl}
b & =q_{32}, & b^{c}, \\
T_{a}^{d} & =T_{a 2}, & & \left(T_{a}^{d}\right)^{c}=-U_{a 1}^{c},
\end{array}
$$

and five charge $2 / 3$ quarks (one of which is the Standard Model $t$-quark), denoted as $t, T_{6}, T_{b}^{(2 / 3)}, T_{5}, T_{a}^{d}$ and $T_{a}^{u}$. It is found that, because there is significant mixing between these states, the mass eigenstates for the charge $2 / 3$ quarks are relatively complicated and are listed up to $\mathcal{O}\left(\frac{v^{2}}{f^{2}}\right)$ in Equations B.38-B.97 of Appendix B. 
The fermion masses were found to be

$$
\begin{aligned}
M_{b}^{2} & =y_{b}^{2} v_{1}^{2}-\frac{2 y_{b}^{2}}{3 \sin ^{2} \beta} \frac{v_{1}^{4}}{f^{2}} \\
M_{t}^{2} & =y_{t}^{2} v_{1}^{2} \\
M_{T_{6}}^{2} & =M_{T_{b}^{(2 / 3)}}^{2}=M_{T_{b}^{(5 / 3)}}^{2}=y_{1}^{2} f^{2} \\
M_{T_{5}}^{2} & =\left(y_{1}^{2}+y_{3}^{2}\right) f^{2}-\frac{9 v_{1}^{2} y_{1}^{2} y_{2}^{2} y_{3}^{2}}{\left(y_{1}^{2}+y_{3}^{2}\right)\left(y_{2}^{2}-y_{3}^{2}\right)} \\
M_{T_{a}^{d}}^{2} & =\left(y_{1}^{2}+y_{2}^{2}\right) f^{2} \\
M_{T_{a}^{u}}^{2} & =\left(y_{1}^{2}+y_{2}^{2}\right) f^{2}+\frac{9 v_{1}^{2} y_{1}^{2} y_{2}^{2} y_{3}^{2}}{\left(y_{1}^{2}+y_{2}^{2}\right)\left(y_{2}^{2}-y_{3}^{2}\right)}
\end{aligned}
$$

where $v_{1}=v \sin \beta, v_{2}=v \cos \beta$ and the top quark Yukawa coupling is given by

$$
y_{t}^{2}=\frac{9 y_{1}^{2} y_{2}^{2} y_{3}^{2}}{\left(y_{1}^{2}+y_{2}^{2}\right)\left(y_{1}^{2}+y_{3}^{2}\right)}
$$

It is evident that if either of the Yukawa couplings $\left(y_{1}, y_{2}, y_{3}\right)$ of Equation 4.58 are zero, then the top quark Yukawa coupling of Equation 4.84 vanishes. This reaffirms that each of the first three terms of Equation 4.58 are required in order to collectively generate a top quark Yukawa coupling.

It should be noted that these fermion mass eigenstates were computed under the assumption that $y_{2} \neq y_{3}$. If $y_{2}=y_{3}$, the masses of the $T_{5}$ and $T_{a}^{u}$ states are degenerate at lowest order. Therefore, the $y_{2}=y_{3}$ case must be treated separately, as it requires a different diagonalization scheme. However, all the results presented in this thesis assume $y_{2} \neq y_{3}$ and the $y_{2}=y_{3}$ case is left for a future study.

It is also evident that the heavy fermions have acquired masses at the scale $f$, whereas the heavy gauge boson masses are at the scale $F>f$. The gauge bosons are therefore heavier than the fermions, thus evading precision electroweak constraints in the gauge sector, while avoiding fine-tuning in the top sector, as desired. The measure 
of this fine-tuning is quantified in Section 4.5 .

\subsection{Radiative Corrections to the Scalar Potential and Fine-Tuning}

The one-loop radiative corrections to the scalar potential of Equation 4.17, due to loops of scalars, gauge bosons and fermions, are calculated by computing the Coleman-Weinberg Potential [51], given by

$$
V_{C W}=\frac{\Lambda^{2}}{32 \pi^{2}} \mathrm{~S} \operatorname{Tr}\left(M^{2}(\Sigma)\right)+\frac{1}{64 \pi^{2}} \mathrm{~S} \operatorname{Tr}\left[M^{4}(\Sigma)\left(\log \left(\frac{M^{2}(\Sigma)}{\Lambda^{2}}\right)-\frac{1}{2}\right)\right]
$$

The Super Trace is defined by

$$
\mathrm{STr} \equiv \sum_{j}(-1)^{2 j}(2 j+1) \operatorname{Tr}\left(M_{j}^{2}\right)=\operatorname{Tr}\left(M_{S}^{2}\right)-2 \operatorname{Tr}\left(M_{F}^{\dagger} M_{F}\right)+3 \operatorname{Tr}\left(M_{V}^{2}\right)
$$

where $M_{S}^{2}, M_{F}^{\dagger} M_{F}$ and $M_{V}^{2}$ are the scalar, fermion and gauge boson mass matrices,

respectively, and $j$ denotes the spin $\left(j=0\right.$ for scalars, $j=\frac{1}{2}$ for fermions and $j=1$ for gauge bosons) [95].

Calculating the one-loop radiative corrections to the scalar potential due to scalars gives

$$
\begin{aligned}
V_{C W S}= & -\frac{3 f^{2} \Lambda^{2}}{8 \pi^{2}}\left[\lambda_{56}\left(f \sigma+\frac{1}{\sqrt{2}} h_{1}^{T} h_{2}+\ldots\right)^{2}+\lambda_{65}\left(f \sigma-\frac{1}{\sqrt{2}} h_{1}^{T} h_{2}+\ldots\right)^{2}\right] \\
& +\frac{\lambda_{56} \lambda_{65}}{16 \pi^{2}} \log \left(\frac{\Lambda^{2}}{m_{\sigma}^{2}}\right)\left[f^{2}\left(h_{1}^{T} h_{1}+h_{2}^{T} h_{2}\right)-\frac{2}{3}\left(h_{1}^{T} h_{1}\right)^{2}\right. \\
& \left.-\frac{2}{3}\left(h_{2}^{T} h_{2}\right)^{2}-\left(h_{1}^{T} h_{1}\right)\left(h_{2}^{T} h_{2}\right)\right]
\end{aligned}
$$

It is evident that there is a quadratically divergent contribution, which is the same 
size but opposite sign as the tree-level contribution of Equation 4.14, so this term does not contribute any dangerous operators. Furthermore, there are no quadratically divergent $\sigma$ tadpoles generated at one loop, which avoids the dangerous singlet problem.

Calculating the one-loop radiative corrections to the scalar potential due to gauge bosons gives

$$
\begin{gathered}
V_{C W V}=\frac{9 g_{A}^{2} g_{B}^{2}}{512 \pi^{2} f^{2}} \log \left(\frac{\Lambda^{2}}{M_{W^{\prime}}^{2}}\right)\left(f^{2}+F^{2}\right)\left(h_{1}^{T} h_{1}+h_{2}^{T} h_{2}+\frac{8}{3} \phi_{a} \phi^{a}\right) \\
-\frac{3 g_{A} g_{B} F^{2}}{64 \pi^{2} f^{2}} \log \left(\frac{\Lambda^{2}}{M_{W^{\prime}}^{2}}\right)\left[\frac{1}{16}\left(h_{1}^{T} h_{1}+h_{2}^{T} h_{2}\right)^{2}+\frac{1}{2} \phi_{a} \phi^{a}\left(h_{1}^{T} h_{1}+h_{2}^{T} h_{2}\right)\right. \\
\left.+\frac{1}{3}\left(\phi_{a} \phi^{a}\right)^{2}\right]
\end{gathered}
$$

It is evident that the logarithmically divergent terms of Equation 4.88 give the largest contribution to the $\phi_{a}$ triplet mass.

Calculating the one-loop radiative corrections to the scalar potential due to the hypercharge gauge boson gives

$$
\begin{aligned}
V_{C W Y}= & \frac{3 g^{\prime 2} \Lambda^{2}}{32 \pi^{2}}\left[\eta_{1}^{2}+\eta_{2}^{2}+\frac{1}{4}\left(h_{1}^{T} h_{1}+h_{2}^{T} h_{2}\right)\right] \\
& -\frac{3 g^{\prime 2} \Lambda^{2}}{32 \pi^{2} f^{2}}\left[\frac{1}{3}\left(\eta_{1}^{2}+\eta_{2}^{2}\right)^{2}+\frac{1}{3}\left(\eta_{1}^{2}+\eta_{2}^{2}\right) \eta_{3}^{2}+\frac{1}{2}\left(\eta_{1}^{2}+\eta_{2}^{2}\right)\left(h_{1}^{T} h_{1}+h_{2}^{T} h_{2}\right)\right] .
\end{aligned}
$$

It is evident that the $\eta_{1}$ and $\eta_{2}$ have acquired quadratically divergent mass terms, which cause the $\eta^{ \pm}=\frac{1}{\sqrt{2}}\left(\eta^{1} \mp i \eta^{2}\right)$ states to be substantially heavier than the neutral $\eta^{0}=\eta^{3}$ state, which only receives a small tree-level mass $m_{\eta^{0}}^{2}=m_{4}^{2}$, as shown in Equation 4.23.

Calculating the one-loop radiative corrections to the scalar potential due to 
fermions gives a finite contribution

$$
V_{C W F}=-\frac{3 f^{2}}{16 \pi^{2}} 9 \frac{y_{1}^{2} y_{2}^{2} y_{3}^{2}}{y_{2}^{2}-y_{3}^{2}} \log \left(\frac{y_{1}^{2}+y_{2}^{2}}{y_{1}^{2}+y_{3}^{2}}\right) h_{1}^{T} h_{1}
$$

It is evident that there are no divergent mass terms from fermion loops.

It is now possible to determine whether these radiative corrections contribute to fine-tuning in the Bestest Little Higgs model. In general, a measure of fine-tuning is the stability of the electroweak Higgs vev under radiative corrections [21]:

$$
\Psi=\left|\frac{\delta v^{2}}{v^{2}}\right|
$$

where $\delta v^{2}$ denotes the shift in $v^{2}$ due to radiative corrections. Since fine-tuning is dominated by a few one-loop diagrams, it is defined as the maximum contribution to Equation 4.91, which in the Bestest Little Higgs model, comes from the radiative corrections to $m_{1}^{2}$ :

$$
\Psi=\left|\frac{\partial v^{2}}{\partial m_{1}^{2}} \frac{\delta m_{1}^{2}}{v^{2}}\right|
$$

Using Equation 4.34 allows this to be rewritten as

$$
\Psi=\frac{\delta m_{1}^{2}}{\lambda_{0} \cos ^{2} \beta v^{2}}
$$

If $\Psi \sim 1$, this indicates that there is no fine-tuning in the model. Determining $\delta m_{1}^{2}$ in each sector from the coefficients of the $h_{1}^{T} h_{1}$ terms in Equations 4.87-4.90, it has been shown that there is no fine-tuning in each sector of the model [21].

It is particularly useful to determine a numerical estimate of the size of the finetuning from fermion loops. Equation 4.90 gives

$$
\delta m_{1}^{2}=-\frac{27 f^{2}}{8 \pi^{2}} \frac{y_{1}^{2} y_{2}^{2} y_{3}^{2}}{y_{2}^{2}-y_{3}^{2}} \log \left(\frac{y_{1}^{2}+y_{2}^{2}}{y_{1}^{2}+y_{3}^{2}}\right)
$$


Using sample parameter values of $v=246 \mathrm{GeV}, \tan \beta=\sqrt{3}, M_{h}=120 \mathrm{GeV}$ and $M_{A^{0}}=500 \mathrm{GeV}$ allows one to solve Equation 4.37 for $\lambda_{0}$, yielding $\lambda_{0}=0.32$. Using $y_{1}=0.5, y_{2}=0.25, y_{3}=1.0$ and $f=700 \mathrm{GeV}$, yields a value of $\Psi_{F}=0.79$, which shows that there is no fine-tuning to the Higgs mass from fermion loops for this choice of parameters. Constraints from fine-tuning on the parameters of the Bestest Little Higgs model, particularly on the Yukawa couplings, $y_{1}, y_{2}, y_{3}$, and $f$, will be examined in greater detail in Section 5.2

\subsection{Summary}

In this chapter, the structure of the Bestest Little Higgs model has been described, and it has been shown that this model solves three problems that occur in most Little Higgs models:

1. A Higgs quartic coupling has been generated through collective symmetry breaking and the scalar sector possesses a custodial symmetry.

2. There are no divergent singlet mass or tadpole terms generated from radiative corrections, which solves the dangerous singlet problem present in other Little Higgs models.

3. It has been explicitly shown that the model predicts the existence of six relatively light top partners, which, contrary to most Little Higgs models, are lighter than the heavy gauge bosons. This allows the gauge bosons to be relatively heavy, consistent with electroweak precision measurements, while avoiding finetuning in the top sector.

In addition to the Standard Model particles, the Bestest Little Higgs model predicts several new scalar and fermion states at the scale $f \sim 1 \mathrm{TeV}$ as well as heavy 
gauge boson partners at the scale $F>f$. There may be interesting signatures in the scalar sector worth studying, but with such light top partners, the collider phenomenology is dominated by the production and decay of the new heavy fermion states, which may be readily produced at the LHC. Therefore, the Feynman rules of this model were derived and implemented in MadGraph5 version 1.1.0 [96] in order to calculate the production cross sections and decay widths of these heavy quarks at the LHC. This analysis of heavy quark production is presented in Chapter 5. 


\section{Chapter 5}

\section{Heavy Quark Production in the Bestest Little Higgs Model at the Large Hadron Collider}

\subsection{Introduction}

The heavy fermion sector of the Bestest Little Higgs model has a rich phenomenology, with four heavy top partners $(+2 / 3$ charge $)$, a heavy bottom partner $(-1 / 3$ charge $)$ and an exotic $+5 / 3$ charge quark, all of which could be relatively light. Discovery of a heavy top partner has been explored in a number of other scenarios [49]. This chapter presents the results of a study [97] of the phenomenology of the heavy fermion sector in the Bestest Little Higgs model at the LHC. Section 5.2 outlines the parameter space of the model with emphasis on constraining the parameters of the fermion sector. Section 5.3 presents calculations of the pair production cross sections for the charge $2 / 3$ heavy top partners, which are then compared with data from the CMS experiment to obtain lower limits on the mass of the lightest top partners. Single production of the charge $2 / 3$ heavy quarks is examined in Section 5.4 and its cross section is compared with that of the pair production process. Production of the exotic 
charge $5 / 3$ heavy quark, $T_{b}^{(5 / 3)}$, is studied in Section 5.5 and its discovery potential at the LHC is determined for a range of parameters. A brief summary of conclusions drawn from this analysis of heavy quark production is given in Section 5.6.

\subsection{Parameters of the Bestest Little Higgs Model}

The Standard Model input parameters used in the calculations of this chapter are as follows:

$$
\begin{aligned}
\alpha_{s}\left(M_{Z}\right) & =0.118 \\
\alpha_{E M}\left(M_{Z}\right) & =\frac{1}{127.9} \\
G_{F} & =1.16637 \times 10^{-5} \mathrm{GeV}^{-2} \\
M_{Z} & =91.1876 \mathrm{GeV} \\
M_{u} & =M_{d}=M_{s}=0 \\
M_{c} & =1.27 \mathrm{GeV} \\
M_{b} & =4.19 \mathrm{GeV} \\
M_{t} & =172 \mathrm{GeV} \\
M_{h} & =120 \mathrm{GeV}
\end{aligned}
$$


The dependent parameters of the Standard Model were calculated from these input parameters as follows:

$$
\begin{aligned}
M_{W} & =\sqrt{\frac{M_{Z}^{2}}{2}+\sqrt{\frac{M_{Z}^{4}}{4}-\frac{\pi \alpha_{E M} M_{Z}^{2}}{\sqrt{2} G_{F}}}}=79.82 \mathrm{GeV} \\
\sin ^{2} \theta_{W} & =1-\frac{M_{W}^{2}}{M_{Z}^{2}}=0.2337 \\
e & =\sqrt{4 \pi \alpha_{E M}}=0.3134 \\
g & =\frac{e}{\sin \theta_{W}}=0.6484 \\
g^{\prime} & =\frac{e}{\cos \theta_{W}}=0.3580 \\
v & =\frac{2 M_{W}}{g}=246.2 \mathrm{GeV}
\end{aligned}
$$

The additional input parameters of the Bestest Little Higgs model used in the calculations of this chapter are as follows:

$$
\begin{aligned}
F & =3 \mathrm{TeV} \\
\tan \beta & =\sqrt{3} \\
\tan \theta_{g} & =1 \\
M_{H^{0}} & =505 \mathrm{GeV} \\
M_{A^{0}} & =M_{H^{ \pm}}=500 \mathrm{GeV} \\
M_{\phi^{0}} & =M_{\phi^{ \pm}}=485 \mathrm{GeV} \\
M_{\eta^{0}} & =10 \mathrm{GeV} \\
M_{\eta^{ \pm}} & =613 \mathrm{GeV}
\end{aligned}
$$

The scale $F$ was chosen to be large enough to ensure that the gauge boson partners are much heavier than the fermion partners under study. Heavy fermion loop 
contributions to the Higgs potential and the lower bound on the Higgs boson mass from LEP suggest that $\tan \beta \gtrsim 1[21]$, so $\tan \beta=\sqrt{3}$ was assumed as in [21]. For simplicity, $\tan \theta_{g}=1$ was assumed, which implies that the gauge couplings $g_{A}$ and $g_{B}$ are equal.

Masses of the scalar states primarily affect the point at which the corresponding decay modes become kinematically accessible. The masses of the $A^{0}, H^{0}, H^{ \pm}, \phi^{0}$, $\phi^{ \pm}$and $\eta^{ \pm}$states are expected to be large enough that they do not significantly affect the results presented in this chapter, while the branching fraction to the $\eta^{0}$ state is too small for its mass parameter to be significant. Therefore, the values $M_{A^{0}}=M_{H^{ \pm}}=500 \mathrm{GeV}$ were chosen and Equations 4.38 and 4.39 were solved to calculate $M_{H^{0}}=505 \mathrm{GeV}$. The masses of the $\phi^{0}, \phi^{ \pm}$and $\eta^{ \pm}$states were calculated from the appropriate mass terms in Equations 4.88 and 4.89. The $\eta^{0}$ state, which couples predominantly to fermions, must not have been seen in low energy flavour factories, implying that its mass must be at least $10 \mathrm{GeV}[21]$.

The mass of the $h$ state may affect the branching ratios of the heavy quarks in the fermion mass region of interest, but recent results from ATLAS and CMS experiments suggest that a Higgs boson mass of $120 \mathrm{GeV}$ is not unreasonable [3,4,34,35]. Small changes to the mass of the Higgs boson have a negligible effect on the results presented here.

For calculations in the fermion sector, it is useful to rewrite the three Yukawa couplings, $y_{1}, y_{2}, y_{3}$, of the Bestest Little Higgs model in terms of the top quark Yukawa coupling, $y_{t}$, and two mixing angles defined by

$$
\begin{aligned}
\tan \theta_{12} & \equiv \frac{y_{1}}{y_{2}} \\
\tan \theta_{13} & \equiv \frac{y_{1}}{y_{3}}
\end{aligned}
$$


The Yukawa couplings, $y_{1}, y_{2}, y_{3}$, can then be expressed as

$$
\begin{aligned}
& y_{1}=\frac{y_{t}}{3 \cos \theta_{12} \cos \theta_{13}} \\
& y_{2}=\frac{y_{t}}{3 \sin \theta_{12} \cos \theta_{13}} \\
& y_{3}=\frac{y_{t}}{3 \cos \theta_{12} \sin \theta_{13}}
\end{aligned}
$$

where

$$
\sin \theta_{1 i}=\frac{y_{1}}{\sqrt{y_{1}^{2}+y_{i}^{2}}}, \quad \cos \theta_{1 i}=\frac{y_{i}}{\sqrt{y_{1}^{2}+y_{i}^{2}}}, \quad(i=2,3)
$$

and $y_{t}=M_{t} / v_{1}$ is the top quark Yukawa coupling defined in Equation 4.84, with $v_{1}=v \sin \beta$. In addition to $\tan \beta$, which is also an input parameter in the scalar and gauge sectors of the model, it is more convenient to use $M_{t}, \tan \theta_{12}$ and $\tan \theta_{13}$ as input parameters rather than $y_{1}, y_{2}$ and $y_{3}$.

The scale $f$ is varied, which yields a range of heavy quark masses. The most stringent theoretical constraint on the heavy quark masses comes from fine-tuning of the Higgs potential due to fermion loops. It is therefore important to determine realistic values for $\tan \theta_{12}$ and $\tan \theta_{13}$, as well as for the scale $f$, that evade these fine-tuning constraints.

The contour plots of Figures 5.1-5.4 show the fine-tuning from fermion loops, $\Psi_{F}$, defined by Equations 4.93-4.94, as a function of $\tan \theta_{12}$ and $\tan \theta_{13}$ for a range of $f$ values. Requiring that $\Psi_{F}<10$ to avoid fine-tuning gives a region of allowed values of $\tan \theta_{12}$ and $\tan \theta_{13}$ for each value of $f$. It is evident that this allowed region shrinks as $f$ increases, as the theory becomes more finely-tuned at these higher scales.

From Equations 4.81 and 4.83 , it is evident that $M_{T_{5}} \leftrightarrow M_{T_{a}^{u}}$ under interchange of $y_{2} \leftrightarrow y_{3}$, so only one side of the central line of $y_{2}=y_{3}$ (or equivalently $\tan \theta_{12}=$ $\left.\tan \theta_{13}\right)$ in parameter space is considered. Points above this line $\left(y_{2}>y_{3}\right)$ exhibit the 


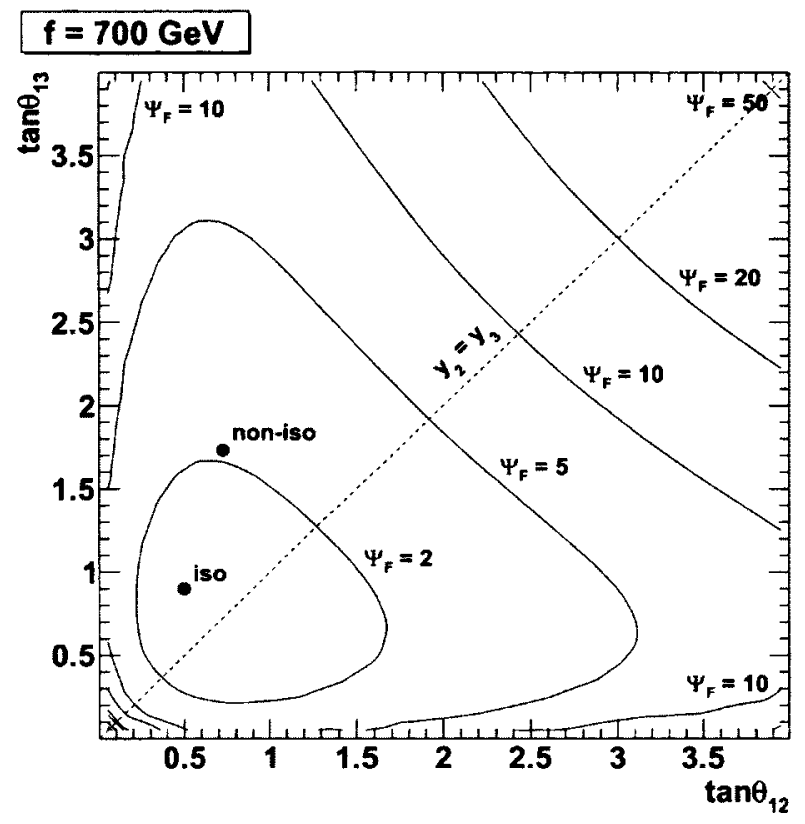

Figure 5.1: Fine-tuning contours from fermion loops, $\Psi_{F}$, as a function of $\tan \theta_{12}$ and $\tan \theta_{13}$ for $f=700 \mathrm{GeV}$, showing placement of the isolated and non-isolated scenarios.

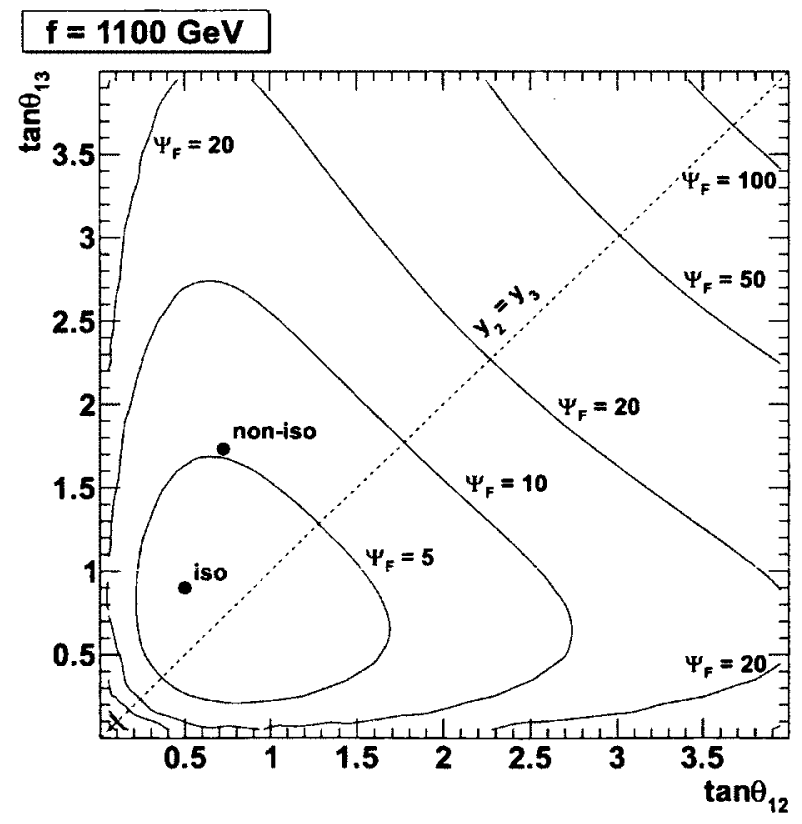

Figure 5.2: Fine-tuning contours from fermion loops, $\Psi_{F}$, as a function of $\tan \theta_{12}$ and $\tan \theta_{13}$ for $f=1100 \mathrm{GeV}$, showing placement of the isolated and non-isolated scenarios. 


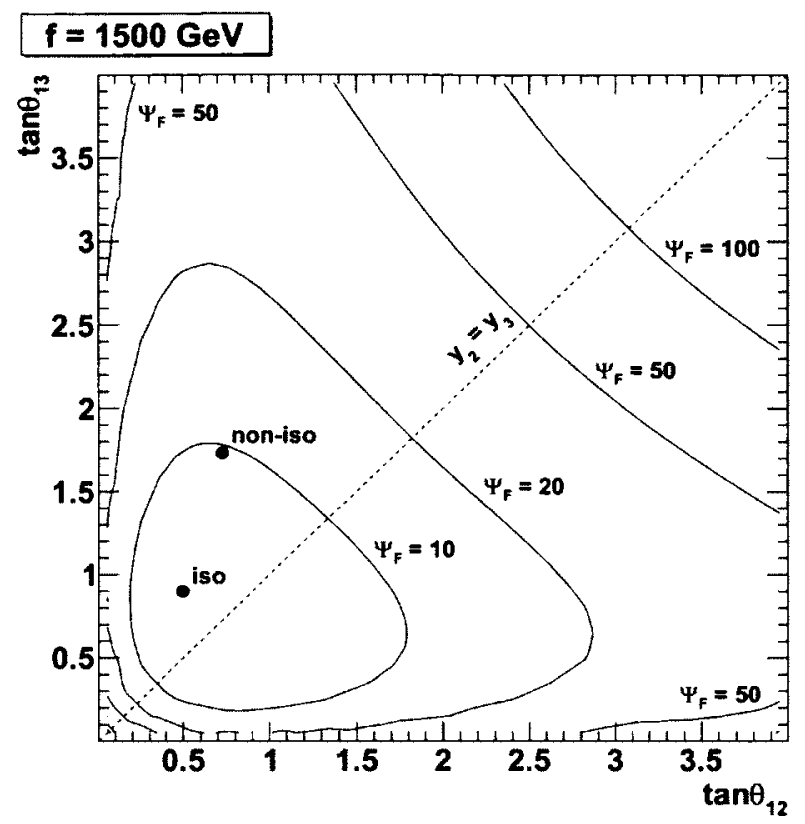

Figure 5.3: Fine-tuning contours from fermion loops, $\Psi_{F}$, as a function of $\tan \theta_{12}$ and $\tan \theta_{13}$ for $f=1500 \mathrm{GeV}$, showing placement of the isolated and non-isolated scenarios.

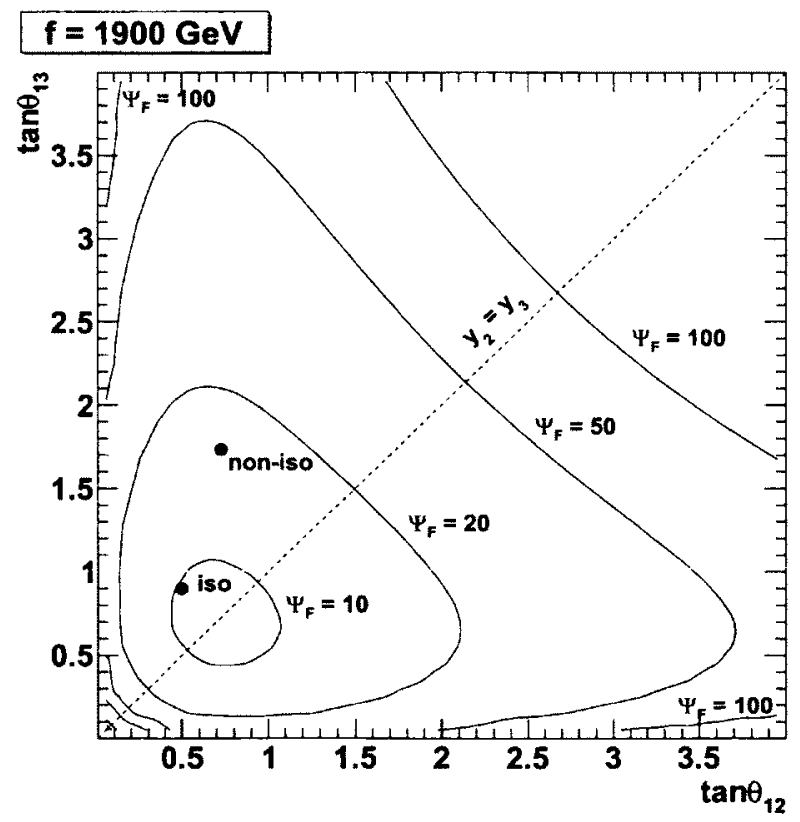

Figure 5.4: Fine-tuning contours from fermion loops, $\Psi_{F}$, as a function of $\tan \theta_{12}$ and $\tan \theta_{13}$ for $f=1900 \mathrm{GeV}$, showing placement of the isolated and non-isolated scenarios. 
mass hierarchy

$$
M_{T_{b}^{(2 / 3)}}=M_{T_{6}}=M_{T_{b}^{(5 / 3)}}<M_{T_{5}}<M_{T_{a}^{d}}<M_{T_{a}^{u}}
$$

whereas points below this line $\left(y_{2}<y_{3}\right)$ exhibit the mass hierarchy

$$
M_{T_{b}^{(2 / 3)}}=M_{T_{6}}=M_{T_{b}^{(5 / 3)}}<M_{T_{a}^{u}}<M_{T_{a}^{d}}<M_{T_{5}}
$$

Since the full range of parameter space for this model is quite large, the analysis of this chapter is restricted to the region $y_{2}>y_{3}$ in which $T_{a}^{u}$ is the heaviest fermion. Two points are chosen in this region of $\left(\tan \theta_{12}, \tan \theta_{13}\right)$ space, for which $\Psi_{F}<10$, denoted the isolated and non-isolated scenarios, given by Equations 5.32 and 5.33, respectively. This nomenclature is defined in the context of the heavy quark mass splittings, which are described in greater detail below.

$$
\begin{aligned}
\text { Isolated : }\left(\tan \theta_{12}, \tan \theta_{13}\right) & =(0.5,0.9) \\
\text { Non - Isolated : }\left(\tan \theta_{12}, \tan \theta_{13}\right) & =\left(\tan \frac{\pi}{5}, \tan \frac{\pi}{3}\right) \approx(0.726,1.732)
\end{aligned}
$$

To impose limits on the scale $f$ in each scenario, it is evident from Figure 5.3 that the non-isolated scenario avoids fine-tuning from fermion loops $\left(\Psi_{F}<10\right)$ up to $f \approx 1500 \mathrm{GeV}$. Meanwhile, Figure 5.4 demonstrates that $f$ is allowed to be larger in the isolated scenario, avoiding fine-tuning up to $f \approx 1900 \mathrm{GeV}$. Setting $\Psi_{F}=10$ and solving for $f$ yields upper limits of $f<1527 \mathrm{GeV}$ for the isolated scenario and $f<1926 \mathrm{GeV}$ for the non-isolated scenario.

The explanation for the shape of these plots is that large values of $\tan \theta_{13}$ (or $\tan \theta_{12}$ ), push the $T_{a}^{u}$ (or $T_{5}$ ) state to be significantly more massive than the others, causing the available region of parameter space to shrink rapidly as the value of $f$ 
increases. Perturbativity requires that $f>v$, although more stringent lower limits on $f$ can be set by experimental constraints from the production of heavy quarks, and vary significantly depending on the values of $\tan \theta_{12}$ and $\tan \theta_{13}$. These lower limits will be examined in Section 5.3.

The reason for naming the isolated and non-isolated scenarios as such is clear when one calculates the heavy fermion masses and determines the allowed decay modes in each scenario. In the non-isolated scenario, the mass splitting between the $T_{5}$ and $T_{6}$ states is relatively small so that the $T_{5}$ decays predominantly to Standard Model particles. On the other hand, in the isolated scenario, the mass splitting between $T_{5}$ and $T_{6}$ is larger, which increases the available decay modes for the $T_{5}$ state through cascade decays to non-Standard Model particles. This has the effect of reducing the branching fraction to the final states considered in this study. The branching ratios for each heavy quark as a function of $f$, or alternatively its mass, are shown in Figures 5.5-5.10 in the two scenarios considered. These branching ratios were calculated using the BRIDGE [86] package of MadGraph.

Further constraints on the values of $f, \tan \beta, \tan \theta_{12}$ and $\tan \theta_{13}$ may arise from loop induced precision electroweak measurements, including deviations to the Standard Model $S$ and $T$ parameters [98], as well as from $D^{0}-\bar{D}^{0}, K^{0}-\bar{K}^{0}, B^{0}-\bar{B}^{0}$ and $B_{s}-\bar{B}_{s}$ mixing and the decay $b \rightarrow s \gamma$ [99-102]. Tree level contributions to both the $S$ and $T$ parameters vanish in the Bestest Little Higgs model, and therefore the model is not strongly constrained by precision electroweak measurements [21]. Since the $T_{a}^{d}$ quark does not mix with the $b$ quark, no limits should be found from $D^{0}-\bar{D}^{0}$ mixing. The couplings of the $T_{5} b W$ and $T_{6} b W$ vertices arise at order $v / f$, prior to implementing the CKM matrix, while the $T_{a}^{u} b W$ and $T_{b}^{(2 / 3)} b W$ vertices arise at order $v^{2} / f^{2}$. Therefore, the primary additional contributions to meson mixing come from 

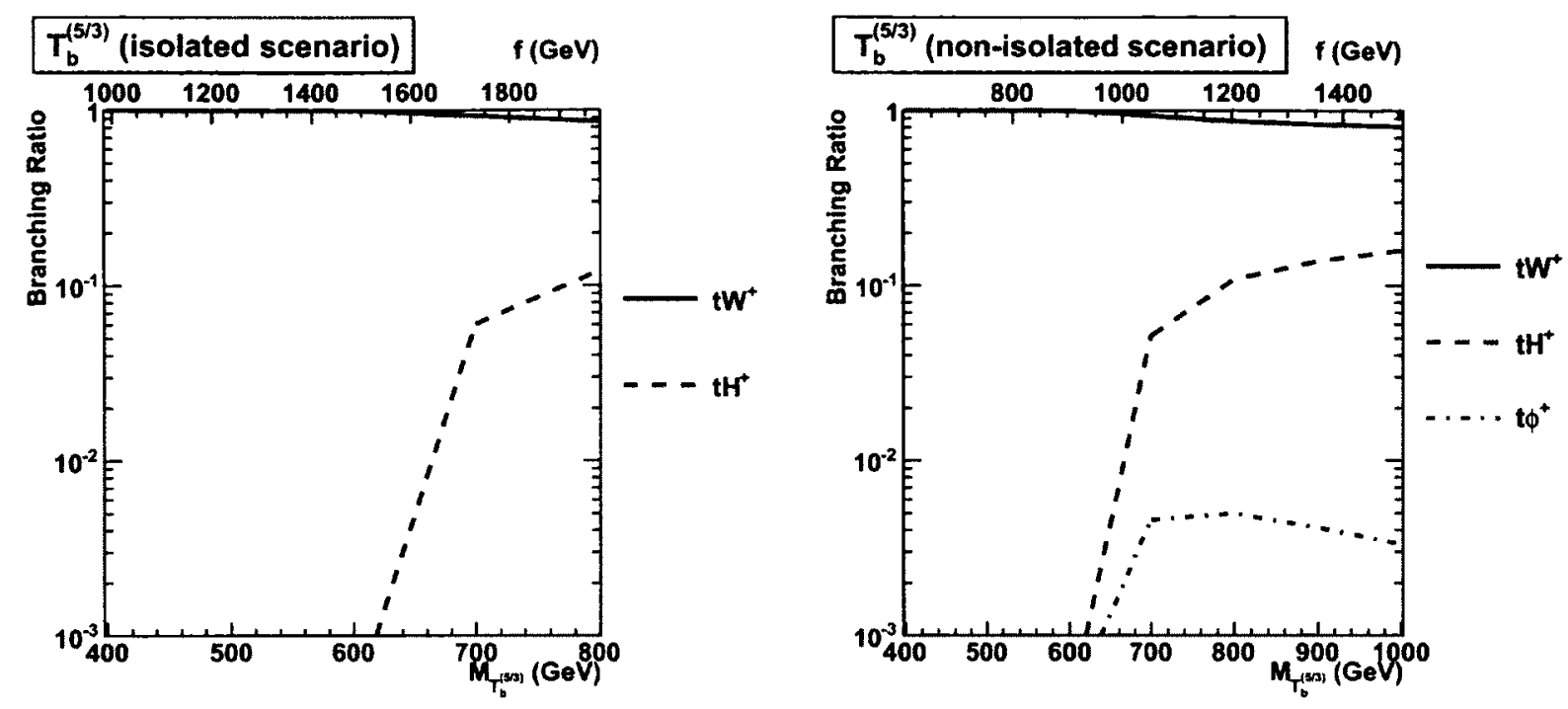

Figure 5.5: $T_{b}^{(5 / 3)}$ branching ratios for all two-body final states with $B R>0.1 \%$. Branching ratios in the isolated (non-isolated) scenario are shown in the plot on the left (right).
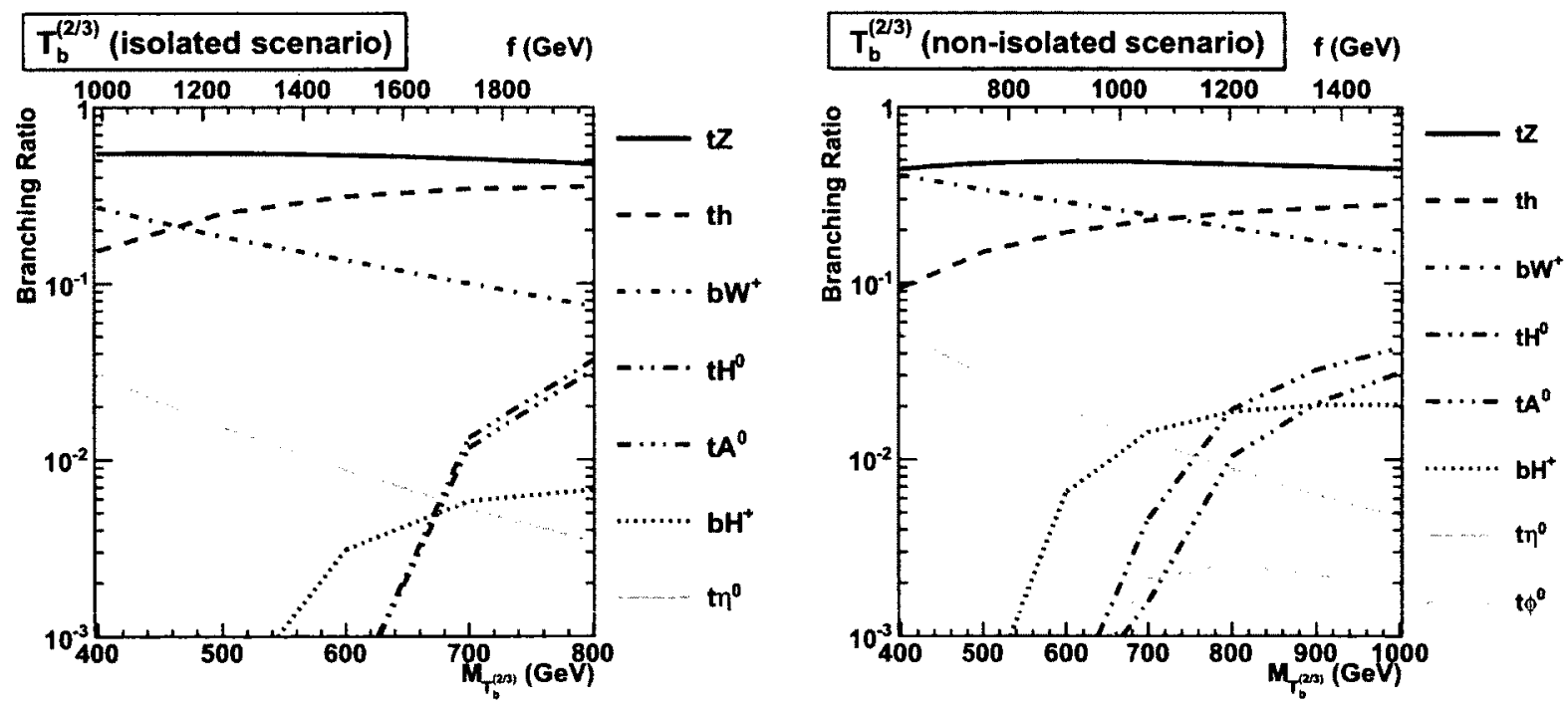

Figure 5.6: $T_{b}^{(2 / 3)}$ branching ratios for all two-body final states with $B R>0.1 \%$. Branching ratios in the isolated (non-isolated) scenario are shown in the plot on the left (right). Note that since the $T_{b}^{(2 / 3)}$ state is among the lightest heavy top quarks, it cannot decay to the other top partners, resulting in similar decay modes for the isolated and non-isolated scenarios. 

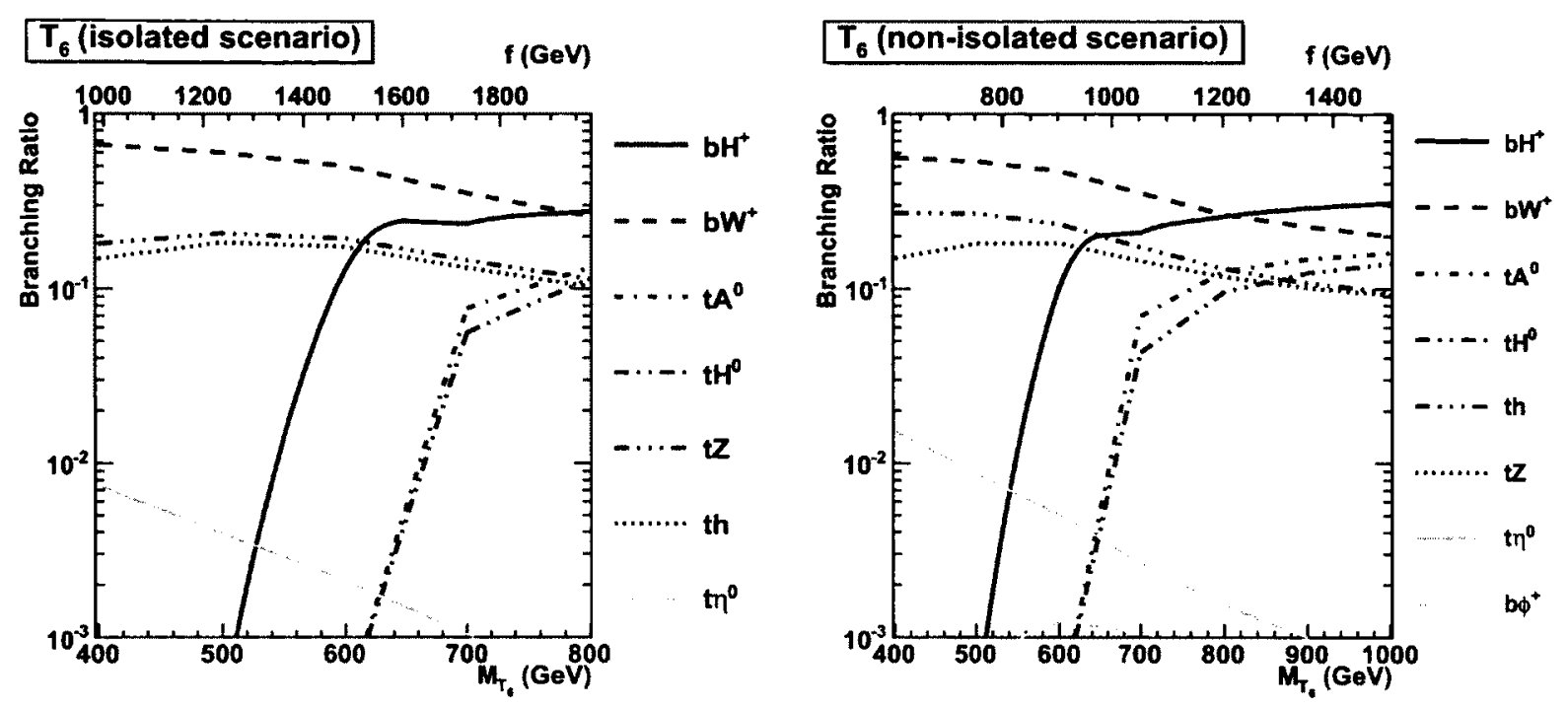

Figure 5.7: $T_{6}$ branching ratios for all two-body final states with $B R>0.1 \%$. Branching ratios in the isolated (non-isolated) scenario are shown in the plot on the left (right). Note that since the $T_{6}$ state is among the lightest heavy top quarks, it cannot decay to the other top partners, resulting in similar decay modes for the isolated and non-isolated scenarios.
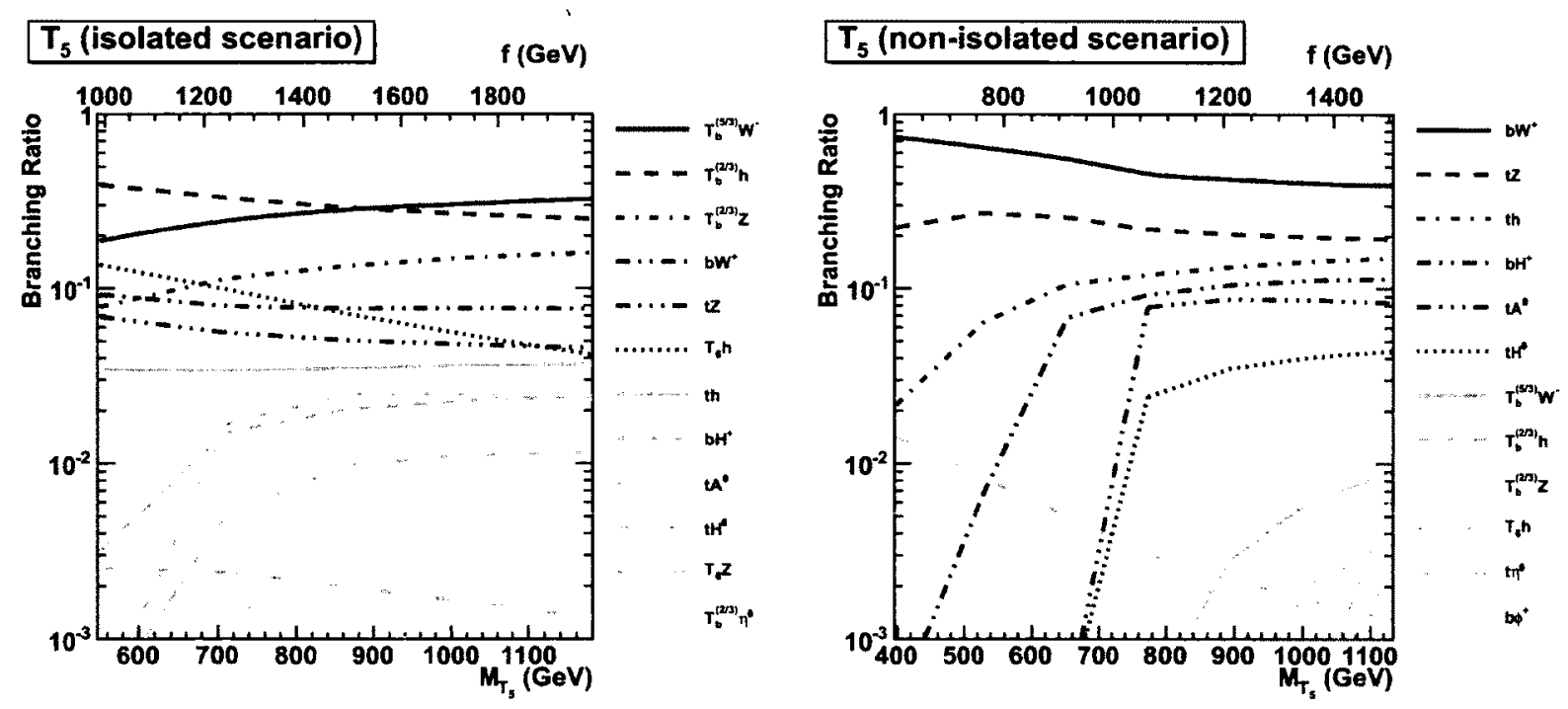

Figure 5.8: $T_{5}$ branching ratios for all two-body final states with $B R>0.1 \%$. Branching ratios in the isolated (non-isolated) scenario are shown in the plot on the left (right). 

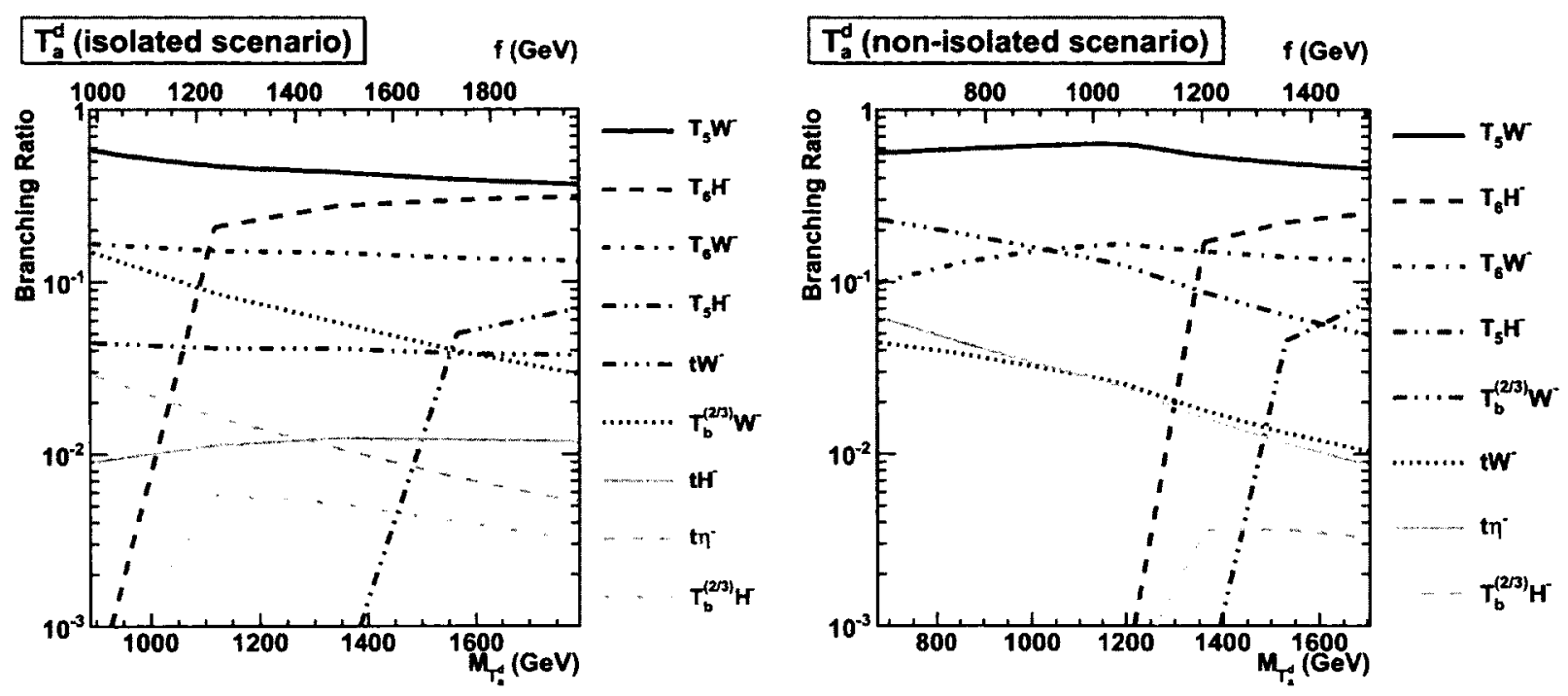

Figure 5.9: $T_{a}^{d}$ branching ratios for all two-body final states with $B R>0.1 \%$. Branching ratios in the isolated (non-isolated) scenario are shown in the plot on the left (right).
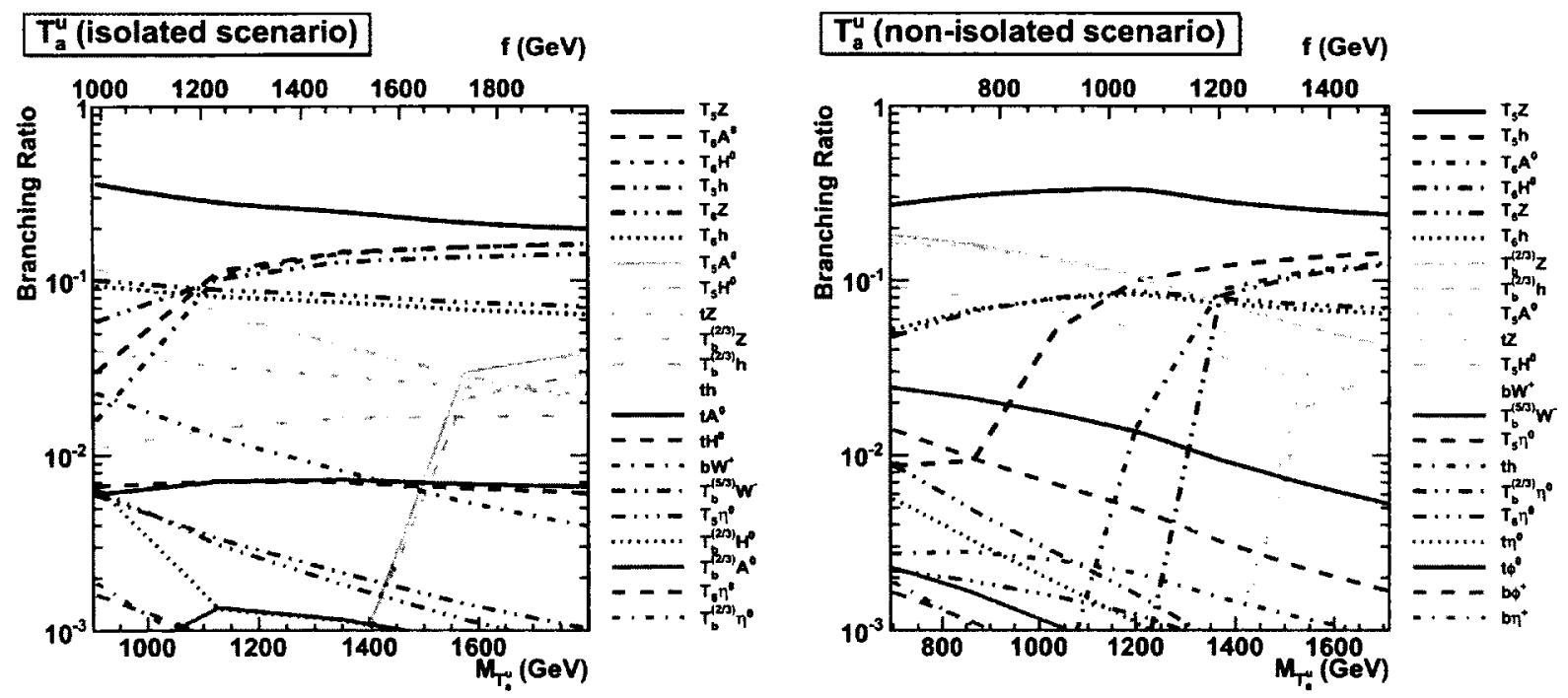

Figure 5.10: $T_{a}^{u}$ branching ratios for all two-body final states with $B R>0.1 \%$. Branching ratios in the isolated (non-isolated) scenario are shown in the plot on the left (right). 
box diagrams involving exchanges of the $T_{5}$ and $T_{6}$ heavy quarks, which are suppressed by powers of $v / f$ relative to the Standard Model contributions. Similarly, the contributions of heavy quark exchanges to the decay $b \rightarrow s \gamma$ are suppressed relative to the Standard Model contributions. Although this may be used to constrain the parameter space of the Bestest Little Higgs model, the scope of this study is direct heavy quark production, and interested readers may review more detailed studies of flavour constraints in [99-102].

\subsection{Pair Production and Discovery Limits of Charge 2/3 Heavy Quarks}

CMS recently presented discovery limits for pair production of a heavy top-like quark at the LHC running at $\sqrt{s}=7 \mathrm{TeV}$, assuming a $100 \%$ branching ratio to the $b W$ final state [103] with an integrated luminosity of $1.14 \mathrm{fb}^{-1}$, and a $100 \%$ branching ratio to the $t Z$ final state [104] with an integrated luminosity of $1.14 \mathrm{fb}^{-1}$ (a previous study used $\left.191 \mathrm{pb}^{-1}[105]\right)$. Since the most recently published results from the ATLAS collaboration are for an integrated luminosity of only $1.04 \mathrm{fb}^{-1}[106]$, they are not considered in this study.

This section presents and compares the results of the total contribution to the $b \bar{b} W^{+} W^{-}$and $t \bar{t} Z Z$ cross sections from the pair production of the lightest heavy top partner states $\left(T_{b}^{(2 / 3)}, T_{6}\right.$ and $\left.T_{5}\right)$ in the Bestest Little Higgs model, given by

$$
\sigma_{t o t}=\sigma_{T_{b}^{(2 / 3)}}+\sigma_{T_{6}}+\sigma_{T_{5}}
$$


Table 5.1: Values of the $A, B, C, D$ and $E$ parameters of Equation 5.38 for heavy quark pair production at the LHC for $\sqrt{s}=7 \mathrm{TeV}$ [107]. The values are evaluated at the scale $\mu=M_{T_{i}}$ using the CTEQ6.6M PDF set [108].

\begin{tabular}{|c|c|c|c|c|c|}
\hline \multicolumn{1}{|c|}{ Process } & $A$ & $B$ & $C$ & $D$ & $E$ \\
\hline$p p \rightarrow q q / g g \rightarrow T_{i} \bar{T}_{i}$ & -0.0684983 & 1.32168 & 1.36903 & -10.4918 & 1.33969 \\
\hline
\end{tabular}

The cross sections for pair production of each heavy quark are given by

$$
\begin{aligned}
\sigma_{T_{b}^{(2 / 3)}} & =\sigma\left(M_{T_{b}^{(2 / 3)}}\right) B R\left(T_{b}^{(2 / 3)} \rightarrow q V\right)^{2} \\
\sigma_{T_{6}} & =\sigma\left(M_{T_{6}}\right) B R\left(T_{6} \rightarrow q V\right)^{2} \\
\sigma_{T_{5}} & =\sigma\left(M_{T_{5}}\right) B R\left(T_{5} \rightarrow q V\right)^{2}
\end{aligned}
$$

where $q=b, t$ and $V=W^{+}, Z$ for the two final states considered. Since the $T_{a}^{u}$ pair production cross section drops off rapidly due to its much higher mass, given in Equation 4.83 , its contribution to both channels considered here is very small and therefore not included. The branching ratios were calculated using the BRIDGE package [86] of MadGraph, and are plotted in Figures 5.5-5.10. The pair production cross sections were calculated using a fit to the Next to Leading Order (NLO) heavy quark production cross section data, using the function [107]

$$
\begin{aligned}
\ln \left[\frac{\sigma\left(M_{T_{i}}, \mu\right)}{\mathrm{pb}}\right]= & A(\mu)\left(\frac{M_{T_{i}}}{\mathrm{TeV}}\right)^{-2}+B(\mu)\left(\frac{M_{T_{i}}}{\mathrm{TeV}}\right)^{-1} \\
& +C(\mu)+D(\mu)\left(\frac{M_{T_{i}}}{\mathrm{TeV}}\right)+E(\mu)\left(\frac{M_{T_{i}}}{\mathrm{TeV}}\right)^{2}
\end{aligned}
$$

where the values of the $A, B, C, D, E$ parameters are listed in Table 5.1 for $\sqrt{s}=7$ $\mathrm{TeV}$ [107] using the CTEQ6.6M PDF set [108] with the scale, $\mu$, set at the top partner mass, $\mu=M_{T_{i}}$. 
The numerical results for the combined cross sections times branching ratios, given by Equations 5.34-5.37, as well as the masses for the $T_{b}^{(2 / 3)}, T_{6}$ and $T_{5}$ quarks, are listed in Tables 5.2 and 5.3. The corresponding results for heavy quark single production, presented in Section 5.4, are also listed in Tables 5.2 and 5.3. The combined pair production cross sections times branching ratios are plotted for both the isolated and non-isolated scenarios in Figure 5.11 for the $b W$ channel and in Figure 5.12 for the $t Z$ channel, versus the mass of the lightest of the heavy top partners (the lightest states are $T_{b}^{(2 / 3)}$ and $T_{6}$ over the range of the figures). Also plotted in these figures are the CMS 95\% confidence level upper limits on the cross section presented in [103,104].

By comparing the observed CMS upper limits on the cross section with the NLO calculations of Equations 5.34-5.38, a lower limit on the mass of the lightest top partner is identified as the mass at which the curves of Figures 5.11 and 5.12 intersect. Lower limits for the mass of the degenerate $T_{b}^{(2 / 3)}$ and $T_{6}$ states are determined to be $369 \mathrm{GeV}$ and $419 \mathrm{GeV}$ for the isolated and non-isolated scenarios, respectively, from the $b W$ channel. From the $t Z$ channel, this lower limit is determined to be $392 \mathrm{GeV}$ and $361 \mathrm{GeV}$, for the same two scenarios respectively. Taking the stronger of the discovery limits in each scenario gives lower mass limits of $392 \mathrm{GeV}$ and $419 \mathrm{GeV}$, corresponding to lower limits of $f=969 \mathrm{GeV}$ and $f=630 \mathrm{GeV}$, for the isolated and non-isolated scenarios, respectively.

Combining these lower limits on the scale $f$ with the upper limits from fine-tuning arguments from fermion loops gives

$$
\begin{aligned}
\text { Isolated : } & 969 \mathrm{GeV}<f<1926 \mathrm{GeV} \\
\text { Non - Isolated : } & 630 \mathrm{GeV}<f<1527 \mathrm{GeV}
\end{aligned}
$$


Table 5.2: Summary of charge $2 / 3$ heavy quark pair and single production cross sections times branching ratios, assuming decays to the $b W$ final state for an LHC centre of mass energy of $\sqrt{s}=7 \mathrm{TeV}$. The single production results listed here are explained in Section 5.4.

\begin{tabular}{|l|l|l|l|l|}
\hline \multicolumn{5}{|c|}{ Isolated Scenario } \\
\hline$f(\mathrm{GeV})$ & $M_{T_{b}^{(2 / 3)}, M_{T_{6}}(\mathrm{GeV})}$ & $M_{T_{5}}(\mathrm{GeV})$ & $\sigma_{\text {pair }}(\mathrm{pb})$ & $\sigma_{\text {single }}(\mathrm{pb})$ \\
\hline 700 & 283.1 & 354.4 & 11.6 & 6.67 \\
800 & 323.5 & 424.8 & 3.59 & 2.33 \\
900 & 364.0 & 492.5 & 1.38 & 1.00 \\
1000 & 404.4 & 558.6 & 0.614 & 0.497 \\
1200 & 485.3 & 687.6 & 0.156 & 0.160 \\
1400 & 566.2 & 814.2 & 0.0418 & 0.0601 \\
1600 & 647.1 & 939.3 & 0.0110 & 0.0240 \\
1800 & 728.0 & 1063.4 & 0.00226 & 0.00878 \\
\hline \multicolumn{5}{|c|}{ Non-Isolated Scenario } \\
\hline$f(\mathrm{GeV})$ & $M_{T_{b}^{(2 / 3)}, M_{T_{6}}(\mathrm{GeV})}$ & $M_{T_{5}}(\mathrm{GeV})$ & $\sigma_{\text {pair }}(\mathrm{pb})$ & $\sigma_{\text {single }}(\mathrm{pb})$ \\
\hline 500 & 332.3 & 304.0 & 7.20 & 12.3 \\
600 & 398.8 & 396.5 & 1.40 & 3.17 \\
700 & 465.3 & 483.5 & 0.387 & 1.10 \\
800 & 531.8 & 567.6 & 0.126 & 0.455 \\
900 & 598.2 & 649.9 & 0.0429 & 0.204 \\
1000 & 664.7 & 730.9 & 0.0149 & 0.0960 \\
1200 & 797.6 & 890.8 & 0.00189 & 0.0242 \\
1400 & 930.6 & 1048.7 & 0.000363 & 0.00797 \\
\hline
\end{tabular}


Table 5.3: Summary of charge $2 / 3$ heavy quark pair and single production cross sections times branching ratios, assuming decays to the $t Z$ final state for an LHC centre of mass energy of $\sqrt{s}=7 \mathrm{TeV}$. The single production results listed here are explained in Section 5.4.

\begin{tabular}{|l|l|l|l|l|}
\hline \multicolumn{5}{|c|}{ Isolated Scenario } \\
\hline$f(\mathrm{GeV})$ & $M_{T_{b}^{(2 / 3)}}, M_{T_{6}}(\mathrm{GeV})$ & $M_{T_{5}}(\mathrm{GeV})$ & $\sigma_{\text {pair }}(\mathrm{pb})$ & $\sigma_{\text {single }}(\mathrm{pb})$ \\
\hline 700 & 283.1 & 354.4 & 1.95 & 2.80 \\
800 & 323.5 & 424.8 & 1.36 & 1.39 \\
900 & 364.0 & 492.5 & 0.736 & 0.665 \\
1000 & 404.4 & 558.6 & 0.409 & 0.344 \\
1200 & 485.3 & 687.6 & 0.130 & 0.110 \\
1400 & 566.2 & 814.2 & 0.0447 & 0.0411 \\
1600 & 647.1 & 939.3 & 0.0162 & 0.0167 \\
1800 & 728.0 & 1063.4 & 0.00570 & 0.00648 \\
\hline \multicolumn{5}{|c|}{ Non-Isolated Scenario } \\
\hline$f(\mathrm{GeV})$ & $M_{T_{b}^{(2 / 3)}}, M_{T_{6}}(\mathrm{GeV})$ & $M_{T_{5}}(\mathrm{GeV})$ & $\sigma_{\text {pair }}(\mathrm{pb})$ & $\sigma_{\text {single }}(\mathrm{pb})$ \\
\hline 500 & 332.3 & 304.0 & 0.688 & 5.03 \\
600 & 398.8 & 396.5 & 0.355 & 1.82 \\
700 & 465.3 & 483.5 & 0.153 & 0.718 \\
800 & 531.8 & 567.6 & 0.0647 & 0.309 \\
900 & 598.2 & 649.9 & 0.0280 & 0.143 \\
1000 & 664.7 & 730.9 & 0.0123 & 0.0685 \\
1200 & 797.6 & 890.8 & 0.00262 & 0.0180 \\
1400 & 930.6 & 1048.7 & 0.000657 & 0.00581 \\
\hline
\end{tabular}




\section{$\mathrm{pp} \rightarrow \mathrm{T} \overline{\mathrm{T}} \rightarrow \mathrm{bW}^{+} \overline{\mathrm{b}} \mathrm{W}^{-}$}

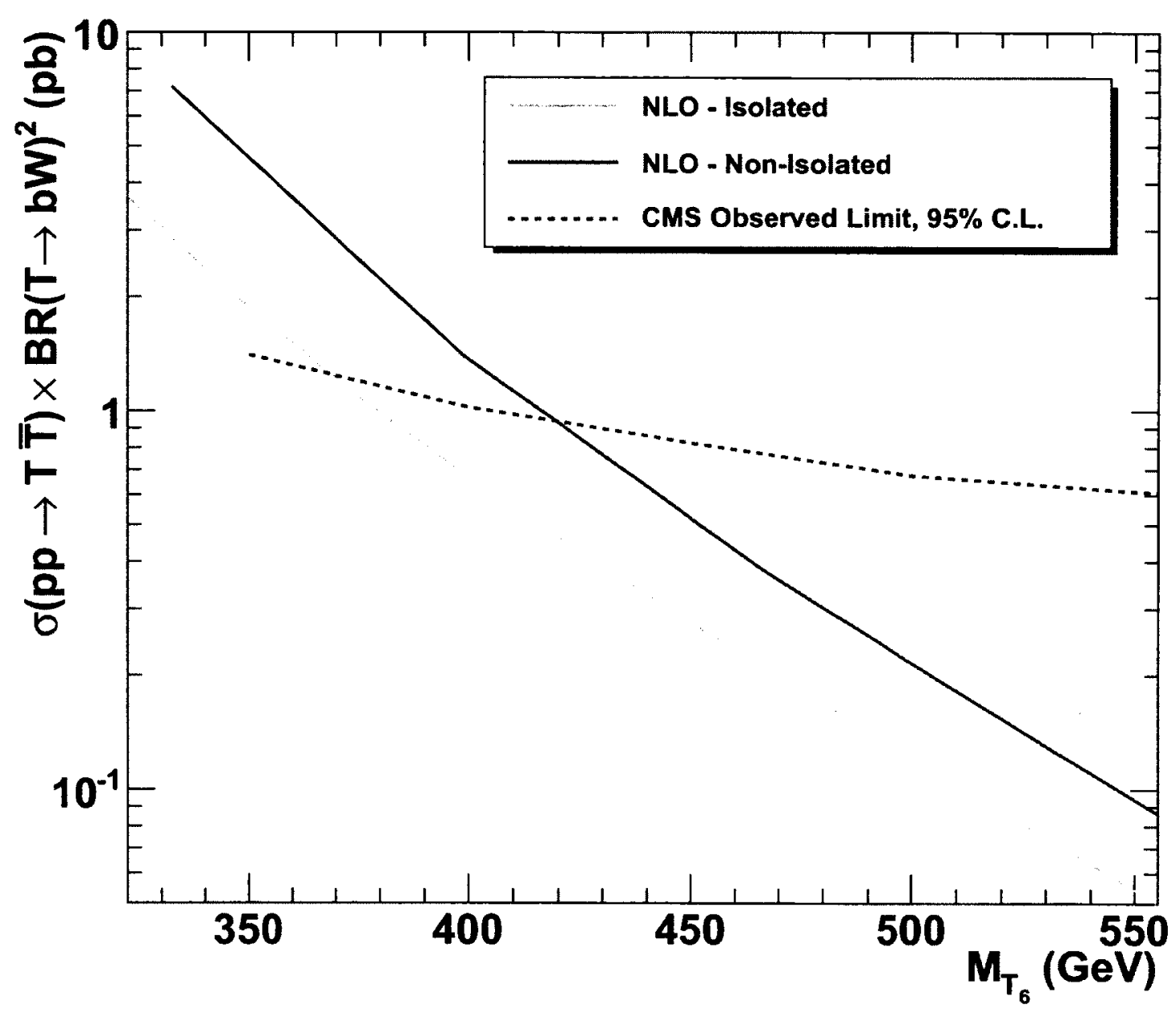

Figure 5.11: Discovery limits on the lightest top partners for the isolated and nonisolated scenarios in the $b W$ final state for an LHC centre of mass energy of $\sqrt{s}=7$ $\mathrm{TeV}$. This plot shows the expected NLO cross section times branching ratio and the CMS 95\% confidence level upper limit [103] in the $b W$ final state. The point at which these curves intersect yields $95 \%$ confidence level lower limits on the mass of the lightest top partner of $369 \mathrm{GeV}$ in the isolated scenario and $419 \mathrm{GeV}$ in the non-isolated scenario. 


\section{$p p \rightarrow T \bar{T} \rightarrow t Z \bar{t} Z$}

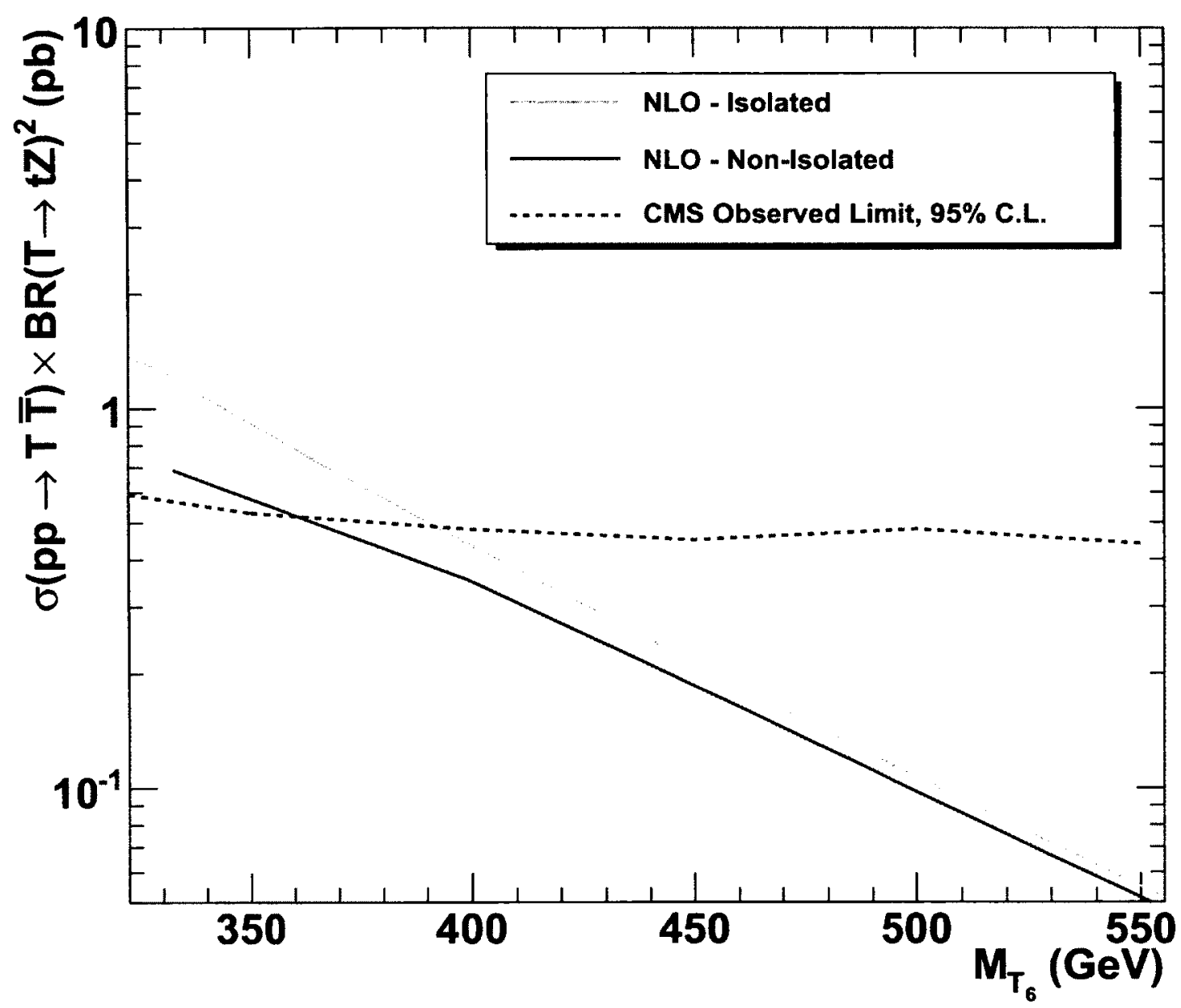

Figure 5.12: Discovery limits on the lightest top partners for the isolated and nonisolated scenarios in the $t Z$ final state for an LHC centre of mass energy of $\sqrt{s}=7$ $\mathrm{TeV}$. This plot shows the expected NLO cross section times branching ratio and the CMS 95\% confidence level upper limit [104] in the $t Z$ final state. The point at which these curves intersect yields $95 \%$ confidence level lower limits on the mass of the lightest top partner of $392 \mathrm{GeV}$ in the isolated scenario and $361 \mathrm{GeV}$ in the non-isolated scenario. 
or, using Equation 4.80, in terms of the mass of the lightest top partners:

$$
\begin{aligned}
\text { Isolated: } & 392 \mathrm{GeV}<M_{T_{6}}=M_{T_{b}^{(2 / 3)}}=M_{T_{b}^{(5 / 3)}}<779 \mathrm{GeV} \\
\text { Non - Isolated : } & 419 \mathrm{GeV}<M_{T_{6}}=M_{T_{b}^{(2 / 3)}}=M_{T_{b}^{(5 / 3)}}<1015 \mathrm{GeV}
\end{aligned}
$$

The lower limit on the mass of the lightest top partners may be further constrained as more LHC data becomes available.

\subsection{Single Production of Charge 2/3 Heavy Quarks}

As first proposed in [39], the charge $2 / 3$ heavy top partners can also be singly produced by t-channel $W$ exchange via the process $q b \rightarrow q^{\prime} T_{i}$ or by s-channel $W$ exchange via the process $q \bar{q}^{\prime} \rightarrow \bar{b} T_{i}$, where $T_{i}=T_{b}^{(2 / 3)}, T_{6}, T_{5}, T_{a}^{u}$ in the Bestest Little Higgs model. Due to the presence of only one heavy top partner in the final state, and hence increased available phase space, the single production cross section falls off much more slowly than pair production as the top partner mass increases. This is also due to the coupling of the heavy quark to the longitudinally polarized $W$ boson, which becomes enhanced at higher energies. However, unlike QCD pair production, this is an electroweak process with a cross section that is more heavily influenced by the model parameters, particularly the Yukawa mixing angles $\left(\tan \theta_{12}, \tan \theta_{13}\right)$.

The NLO single production cross sections of the heavy top partners were calculated using the fitting function [107]

$$
\ln \left[\frac{\sigma\left(M_{T_{i}}, \mu\right)}{\mathrm{pb}}\right]=A(\mu)\left(\frac{M_{T_{i}}}{\mathrm{TeV}}\right)^{-1}+B(\mu)+C(\mu)\left(\frac{M_{T_{i}}}{\mathrm{TeV}}\right)
$$

where the values of the $A, B$, and $C$ parameters are listed in Table 5.4 for $\sqrt{s}=7$ 
Table 5.4: Values of the $A, B, C$ parameters of Equation 5.43 for heavy quark single production processes at the LHC for $\sqrt{s}=7 \mathrm{TeV}$ [107]. The values are evaluated at the scale $\mu=M_{T_{i}}$ using the CTEQ6.6M PDF set [108].

\begin{tabular}{|c|c|c|c|}
\hline Process & $A$ & $B$ & $C$ \\
\hline$p p \rightarrow q b \rightarrow q^{\prime} T_{i}$ & $0.72823_{-0.01262}^{+0.00130}$ & $1.66318_{-0.00630}^{+0.03563}$ & $-3.38334_{-0.04100}^{+0.05251}$ \\
\hline$p p \rightarrow q \bar{b} \rightarrow q^{\prime} \bar{T}_{i}$ & $0.77742_{-0.00158}^{+0.00580}$ & $0.86229_{-0.03096}^{+0.04447}$ & $-3.57353_{-0.05169}^{+0.05666}$ \\
\hline$p p \rightarrow q \bar{q}^{\prime} \rightarrow \bar{b} T_{i}$ & $1.37193_{-0.01703}^{+0.00541}$ & $-3.44671_{-0.05685}^{+0.03710}$ & $-5.22956_{-0.01643}^{+0.02415}$ \\
\hline$p p \rightarrow q \bar{q}^{\prime} \rightarrow b \bar{T}_{i}$ & $1.79403_{-0.02359}^{+0.00423}$ & $-5.40590_{-0.06414}^{+0.02628}$ & $-4.81694_{-0.01401}^{+0.02980}$ \\
\hline
\end{tabular}

$\mathrm{TeV}[107]$ using the CTEQ6.6M PDF set [108] with the scale, $\mu$, set at the top partner mass, $\mu=M_{T_{i}}$.

For simplicity, the function of Equation 5.43 assumes that the $W T_{i} b$ vertices are the same as the Standard Model $W t b$ vertex. The cross sections computed here were therefore rescaled using the correct $W T_{i} b$ vertices of the Bestest Little Higgs model. The CTEQ6.6M PDF set [108] was used, and branching ratios of the heavy top partners to third generation fermions and Standard Model gauge bosons were calculated using BRIDGE [86]. The resulting cross section for single production via t-channel $W$ exchange is given by,

$$
\sigma_{T_{i}}=\sigma\left(p p \rightarrow q b \rightarrow q^{\prime} T_{i}\right) B R\left(T_{i} \rightarrow q_{3} V\right)
$$

where $q=u, c ; q^{\prime}=d, s$; and $q_{3}=b, t$ and $V=W^{+}, Z$ for the two final states considered. This cross section was calculated for $T_{i}=T_{b}^{(2 / 3)}, T_{6}, T_{5}$. The $T_{a}^{u}$ state is much heavier for the parameters considered, and was not included as its single production cross section is much smaller.

The charge-conjugate process $q \bar{b} \rightarrow q^{\prime} \bar{T}_{i}$ is also included. This does not have the same cross section as the $T_{i}$ single production process due to the different parton 
distribution functions of the initial-state quarks. Its cross section is given by,

$$
\sigma_{\bar{T}_{i}}=\sigma\left(p p \rightarrow q \bar{b} \rightarrow q^{\prime} \bar{T}_{i}\right) B R\left(\bar{T}_{i} \rightarrow \bar{q}_{3} V\right)
$$

where, in this case, $q=d, s ; q^{\prime}=u, c$; and $\bar{q}_{3}=\bar{b}, \bar{t}$ and $V=W^{-}, Z$ for the two final states considered.

The cross section for single production is thus given by the sum of all contributions from heavy top partners with similar detector signatures $\left(q^{\prime} q_{3} V\right.$ and $\left.q^{\prime} \bar{q}_{3} V\right)$ :

$$
\sigma_{\text {single }}=\sigma_{T_{b}^{(2 / 3)}}+\sigma_{T_{6}}+\sigma_{T_{5}}+\sigma_{\bar{T}_{b}^{(2 / 3)}}+\sigma_{\bar{T}_{6}}+\sigma_{\bar{T}_{5}}
$$

The cross section of Equation 5.46 is plotted in Figures 5.13 and 5.14 versus $f$ (or alternatively, versus the mass of the lightest top partners) for the isolated and non-isolated scenarios respectively, for both the $b W$ and $t Z$ final states, again using the parameters of Equations 5.1-5.23.

Tables 5.2 and 5.3 present the numerical results for single production via t-channel $W$ exchange in the two scenarios, along with the cross sections for pair production. Single production cross sections are generally larger than the pair production cross sections for larger top-partner masses, as expected. A further advantage of studying single production is that it does not suffer from the large $t \bar{t}$ backgrounds that exist for heavy top pair production.

As a further check of these single production results, the leading order (LO) cross sections for $p p \rightarrow q b \rightarrow q^{\prime} T_{i}$ were calculated for $T_{i}=T_{b}^{(2 / 3)}, T_{5}, T_{6}$, using MadGraph5 version 1.1.0 [96] with the CTEQ6.6M PDF sets [108]. These LO cross sections were compared to the NLO cross sections calculated using the fit of Equation 5.43. The resulting K-factors (ratio of NLO to LO cross sections) are shown in Figure 5.15 and are similar to those found in [109]. However, as explained in [107], the fit that was 


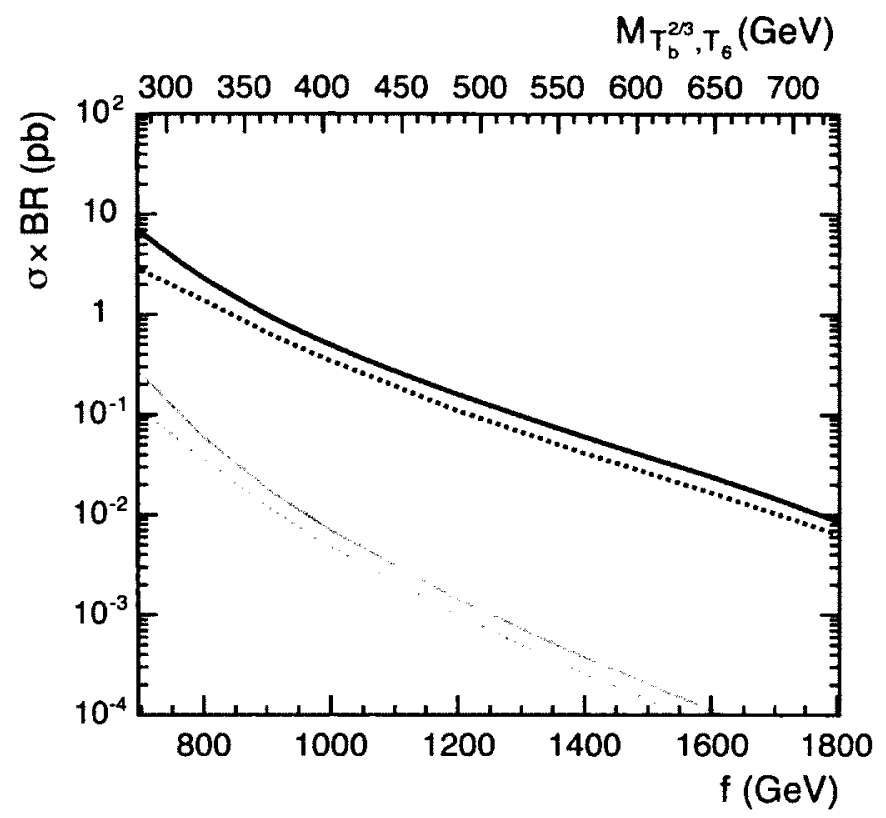

Figure 5.13: Combined single production NLO cross sections times branching ratios in the isolated scenario for $p p \rightarrow q b \rightarrow q^{\prime} T_{i}$ (black) and $p p \rightarrow q \bar{q}^{\prime} \rightarrow \bar{b} T_{i}$ (grey), for the $b W$ (solid) and $t Z$ (dashed) decay channels at $\sqrt{s}=7 \mathrm{TeV}$. Cross sections include the charge conjugate processes.

used to obtain the NLO cross sections is less valid at lower masses, resulting in a larger K-factor in the low mass range. Similar to the pair production case of Equation 5.38, one could improve the fit in the low mass range by increasing the number of terms in the polynomial expansion of the fitting function, as stated in [107]. However, this is beyond the scope of this thesis since much of this low mass range has been ruled out based on the discovery limits calculated in Section 5.3.

The charge $2 / 3$ heavy top partners can also be singly produced by s-channel $W$ exchange via the processes $q \bar{q}^{\prime} \rightarrow \bar{b} T_{i}$ and $q \bar{q}^{\prime} \rightarrow b \bar{T}_{i}$, where the states being considered are $T_{i}=T_{b}^{(2 / 3)}, T_{6}, T_{5}$. The NLO cross section for these processes was calculated in the same manner as the t-channel $W$ exchange process and is also plotted in Figures 5.13 and 5.14 for the isolated and non-isolated scenarios, respectively, for both the $b W$ and $t Z$ final states, again for $\sqrt{s}=7 \mathrm{TeV}$. It is evident that the total 


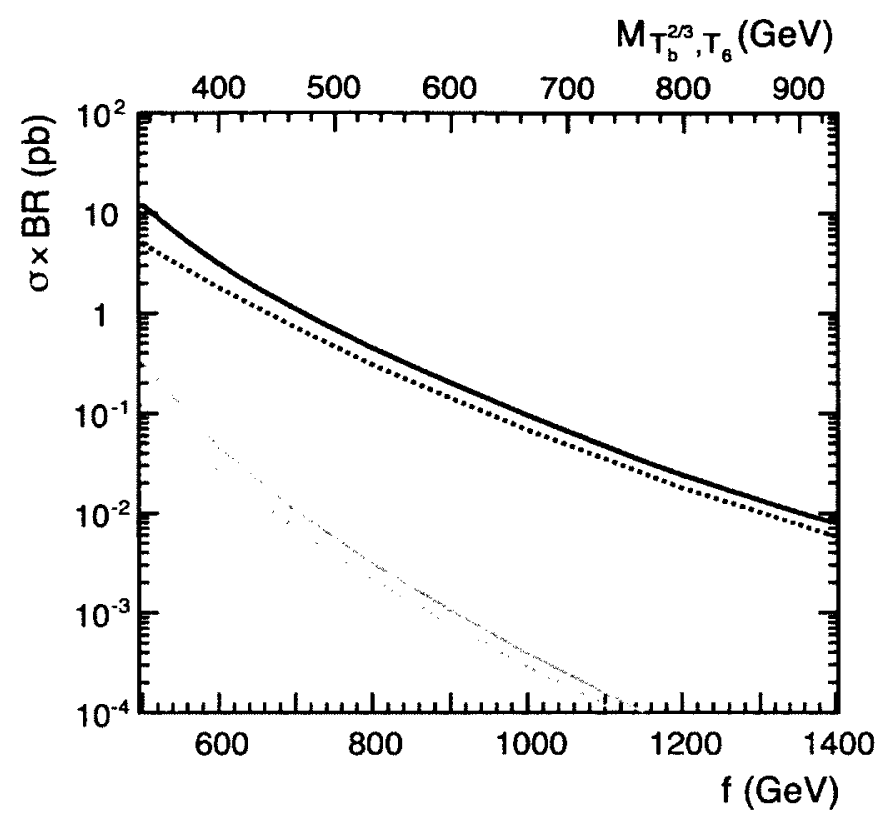

Figure 5.14: Combined single production NLO cross sections times branching ratios in the non-isolated scenario for $p p \rightarrow q b \rightarrow q^{\prime} T_{i}$ (black) and $p p \rightarrow q \bar{q}^{\prime} \rightarrow \bar{b} T_{i}$ (grey), for the $b W$ (solid) and $t Z$ (dashed) decay channels at $\sqrt{s}=7 \mathrm{TeV}$. Cross sections include the charge conjugate processes.

cross section for $p p \rightarrow q \bar{q}^{\prime} \rightarrow \bar{b} T_{i}$ and $p p \rightarrow q \bar{q}^{\prime} \rightarrow b \bar{T}_{i}$ is roughly two orders of magnitude lower than the total cross section for $p p \rightarrow q b \rightarrow q^{\prime} T_{i}$ and $p p \rightarrow q \bar{b} \rightarrow q^{\prime} \bar{T}_{i}$. This suggests that t-channel $W$ exchange is the most promising process to study single top partner production at the LHC. However, a full analysis of the detector signatures and backgrounds in this channel is required to determine discovery capabilities in this channel.

ATLAS has recently measured a single $t$-quark production cross section of $90_{-22}^{+32} \mathrm{pb}$ in the t-channel mode, after cuts, using $0.70 \mathrm{fb}^{-1}$ of data at $\sqrt{s}=7 \mathrm{TeV}$ [110]. More luminosity is needed to reduce the uncertainty on this cross section and, since it is larger than the cross sections for single production of heavy top partners shown in Tables 5.2 and 5.3, harder cuts will be required to reduce this single top-quark background to a manageable level. The dominant Standard Model backgrounds to 


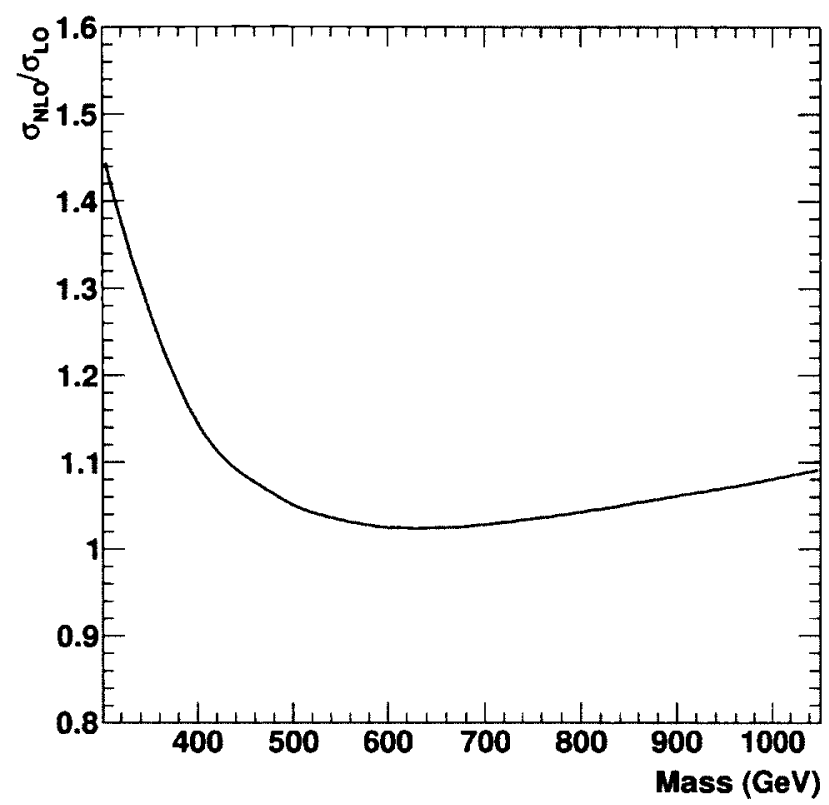

Figure 5.15: Ratio of NLO and LO cross sections for single production via t-channel $W$ exchange for $\sqrt{s}=7 \mathrm{TeV}$, as a function of the top partner mass. Only one curve is shown as the $\mathrm{K}$-factors were found to be identical for every scenario.

single heavy top production are single $t$-quark production in the $T_{i} \rightarrow b W$ channel, $W Z, Z Z$ and $t b Z$ production in the $T_{i} \rightarrow t Z$ channel, and $t \bar{t}$ production in the $T_{i} \rightarrow$ th channel as a $125 \mathrm{GeV}$ Higgs decays predominantly to $b \bar{b}$. As techniques for reducing these backgrounds to single heavy top quark production are already well established [49], they are not considered here and left for a future study. Instead, the more interesting signature from production and decay of the exotic charge $5 / 3$ heavy quark, $T_{b}^{(5 / 3)}$, is studied in detail in Section 5.5. 


\subsection{Single Associated Production of the Charge 5/3 Heavy Quark, $T_{b}^{(5 / 3)}$}

\subsubsection{Introduction}

Perhaps the most novel feature of the fermion sector of the Bestest Little Higgs model is the prediction of an exotic charge $5 / 3$ heavy quark, denoted as the $T_{b}^{(5 / 3)}$ state, as well as a charge $-1 / 3$ heavy quark, denoted by $T_{a}^{d}$. These can be produced at the LHC in association with a top quark and a light quark jet

$$
\begin{aligned}
& p p \rightarrow q g \rightarrow T_{b}^{(5 / 3)} \bar{t} q^{\prime} \\
& p p \rightarrow q g \rightarrow T_{a}^{d} \bar{t} q^{\prime} .
\end{aligned}
$$

From Figure 5.5, it is evident that the $T_{b}^{(5 / 3)}$ almost always decays to $t W^{+}$, where the dominant top quark decay mode is $t \rightarrow b W^{+}$. Similarly, it can be seen from Figure 5.9 that the $T_{a}^{d}$ tends to decay to a charge $2 / 3$ quark and a $W^{-}$(or $H^{-}$) boson. As pointed out in $[111,112]$, focusing on leptonic decays of the like-sign $W$ bosons yields a very clean signature of same-sign dileptons in the final state.

The discovery potential in the same-sign dilepton channel from pair production of charge $5 / 3$ and charge $-1 / 3$ heavy quarks has been investigated by Contino and Servant [111]. Since heavy quark pair production is purely a QCD process, independent of the parameters in the electroweak sector, it is not necessary to repeat their analysis for $T_{b}^{(5 / 3)}$ and $T_{a}^{d}$ pair production in the Bestest Little Higgs model. However, single associated production of these top partners is an electroweak process, highly dependent on the parameters of the electroweak sector, and becomes comparable to the QCD production process in the high-mass range. It is therefore worth studying single

associated production of $T_{b}^{(5 / 3)}$ and $T_{a}^{d}$ in the Bestest Little Higgs model. However, 
since the $T_{a}^{d}$ state is significantly heavier than the $T_{b}^{(5 / 3)}$ state, its contribution to the same-sign dilepton channel can be neglected. Therefore, in this section, the signals and backgrounds for single associated production of $T_{b}^{(5 / 3)}$ in the same-sign dilepton channel are investigated in order to determine the discovery potential for this exotic charge $5 / 3$ heavy quark at the LHC.

\subsubsection{Signal and Backgrounds}

The process for single associated production of the $T_{b}^{(5 / 3)}$ heavy quark with decays to same-sign dileptons is shown in Figure 5.16. This figure shows the $\ell^{+} \ell^{+}$channel, although the $\ell^{-} \ell^{-}$channel, in which the charge-conjugate $\bar{T}_{b}^{(5 / 3)}$ is produced, is also included in this analysis. Due to the different parton distribution functions of the initial state quarks, the cross section for the $\ell^{+} \ell^{+}$channel is roughly twice as large as the cross section for the $\ell^{-} \ell^{-}$channel. Not to enter into the subtleties of $\tau$ reconstruction, only decays to electrons and muons $(\ell=e, \mu)$ are considered. As $\tau$ leptons decay almost instantly and their decays generally involve missing energy, they are very difficult to reconstruct from their decay products at the LHC. Assuming they could be efficiently reconstructed, considering decays to $\tau$ leptons as well could increase the signal cross section, but this would also increase the background cross sections by roughly the same factor. This may improve the significance, $S / \sqrt{B}$, so to obtain a more conservative estimate of the $T_{b}^{(5 / 3)}$ discovery potential, decays to $\tau$ leptons are not considered.

Due to the neutrinos in the final state, which pass through the detector without being detected, there is a large amount of missing transverse energy in the final state. There are also multiple jets, most of which arise through cascade decays of the heavy 


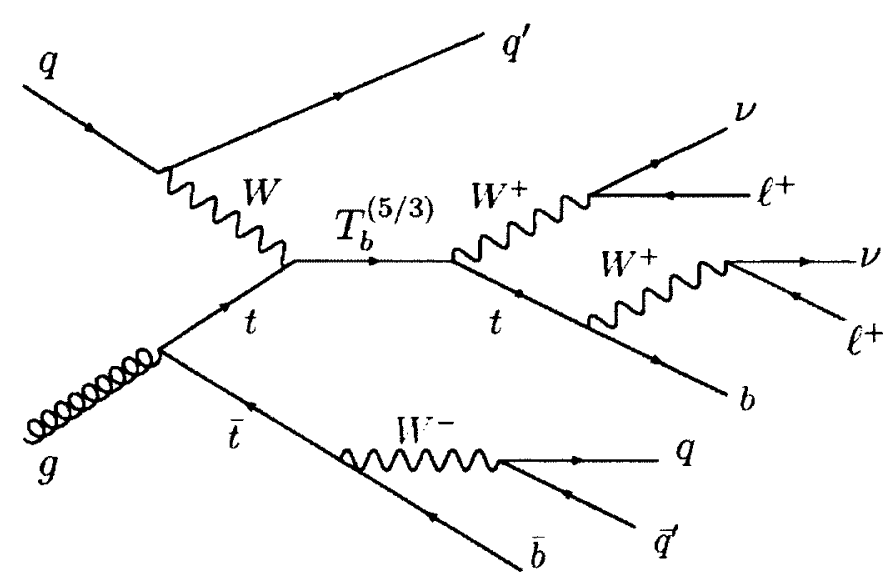

Figure 5.16: Production of the exotic charge $5 / 3$ heavy quark, $T_{b}^{(5 / 3)}$, in association with a $\bar{t}$ quark and a light quark jet, with decays to same-sign dileptons in the final state.

particles. The signal of interest is therefore

$$
p p \rightarrow \ell^{ \pm} \ell^{ \pm}+\mathbb{E}_{T}+n \text { jets }, \quad(\ell=e, \mu)
$$

The quark jet emanating from one of the initial partons tends to be emitted in the forward region of the detector. Therefore, a forward jet tag will be useful in improving the signal to background ratio.

The backgrounds can be divided into four categories:

1. True Backgrounds: processes in which exactly two same-sign leptons are produced.

$$
\begin{aligned}
& p p \rightarrow W^{ \pm} W^{ \pm}+2,3 \text { jets } \\
& p p \rightarrow W^{ \pm} W^{ \pm} W^{\mp}+0,1,2 \text { jets } \\
& p p \rightarrow t \bar{t} W^{ \pm}+0,1,2 \text { jets } \\
& p p \rightarrow t \bar{t} W^{ \pm} W^{\mp}+0,1 \text { jets }
\end{aligned}
$$


2. Charge Misidentification Backgrounds: processes in which two opposite-sign leptons are produced, but the charge of one of the leptons is misidentified.

$$
\begin{aligned}
& p p \rightarrow t \bar{t}+0,1,2 \text { jets } \\
& p p \rightarrow W^{ \pm} W^{\mp}+0,1,2 \text { jets } \\
& p p \rightarrow Z^{*} / \gamma^{*}+0,1,2,3 \text { jets }
\end{aligned}
$$

The cross sections for these processes are generally much larger than the cross sections of the true backgrounds listed above. However, in this case, they are suppressed by the lepton charge misidentification probability, $\epsilon_{\text {charge }}$, which is typically on the order of $10^{-3}$ and can be even smaller for muons [113].

3. Fake Lepton Backgrounds: processes in which only one lepton is produced, but a jet is misidentified as a second same-sign lepton. In the same-sign dilepton channel, there is only one such relevant background:

$$
p p \rightarrow W^{ \pm}+1,2,3 \text { jets }
$$

As with the charge misidentification backgrounds, the cross section for this process is normally much larger than those of the true backgrounds. However, in this case, it is suppressed by the fake rate, $\epsilon_{f a k e}$, which is typically on the order of $10^{-4}$ with a corresponding efficiency of roughly $90 \%$ for reconstructing electrons and an even higher efficiency for reconstructing muons $[87,113,114]$.

4. Missing Lepton Backgrounds: processes in which more than two leptons are produced, but one of them is lost, either outside the detector $(|y|>2.5)$ or produced with a $p_{T}$ below threshold, which is set at $10 \mathrm{GeV}$. In the same-sign 
dilepton channel, there is only one such relevant background:

$$
p p \rightarrow W^{ \pm} Z+0,1,2 \text { jets }
$$

where one of the leptons from the $Z \rightarrow \ell^{+} \ell^{-}$decay is not detected. Despite the geometrical suppression for losing a lepton, this process is comparable to the $W^{ \pm} W^{ \pm}$background since the latter final state is necessarily produced in associated with at least two jets, and therefore at higher order in $\alpha$. On the other hand, the $W^{ \pm} W^{ \pm} Z$ process is subdominant and can be neglected compared to the $W^{ \pm} W^{ \pm} W^{\mp}$ background, which is not necessarily produced in association with two or more jets and hence involves a larger number of Feynman diagrams.

\subsubsection{Calculations}

The Feynman rules for the Bestest Little Higgs model were calculated and implemented in MadGraph5 version 1.1.0 [96] and weighted signal and background events were then generated. MadGraph generates tree-level matrix elements and then generates events using Monte Carlo phase space integration. The generated events were analyzed, including implementing kinematic cuts in order to determine the discovery potential in the same-sign dilepton channel. One advantage of MadGraph5 over MadGraph4 is that it is possible to implement the decay chains directly in the process input file without using the DECAY package after generating events. The branching ratios were calculated using BRIDGE [86], as shown in Figures 5.5-5.10 and the CTEQ6.6M parton distribution functions [108] were used. No K-factors have been included, since those for the backgrounds are not all available.

The Standard Model input parameters of Equations 5.1-5.9 were used, along with the Bestest Little Higgs model input parameters of Equations 5.16-5.23. Both the isolated and non-isolated scenarios were considered, corresponding to $\left(\tan \theta_{12}, \tan \theta_{13}\right)=$ 
$(0.5,0.9)$ and $\left(\tan \theta_{12}, \tan \theta_{13}\right)=\left(\tan \frac{\pi}{5}, \tan \frac{\pi}{3}\right) \approx(0.726,1.732)$, respectively. Three values of the $T_{b}^{(5 / 3)}$ mass were chosen according to the constraints of Equations 5.415.42: $M_{T_{b}^{(5 / 3)}}=400 \mathrm{GeV}, 600 \mathrm{GeV}$ and $800 \mathrm{GeV}$.

The sum of the $T_{b}^{(5 / 3)}$ and $\vec{T}_{b}^{(5 / 3)}$ production cross sections with decays to same-sign dileptons is plotted in Figure 5.17 for both the isolated and non-isolated scenarios, at LHC centre of mass energies of $\sqrt{s}=7 \mathrm{TeV}, 8 \mathrm{TeV}$ and $14 \mathrm{TeV}$. One can see that the cross section at $\sqrt{s}=7 \mathrm{TeV}$ is, at best, $\sim 1 \mathrm{fb}$ for $M_{T_{b}^{(5 / 3)}}=400 \mathrm{GeV}$. As the early years of LHC running are expected to produce $\sim 10 \mathrm{fb}^{-1}$ of integrated luminosity per year, this translates to $\sim 10$ signal events per year. Implementing kinematic cuts to improve the signal to background ratio will reduce the number of signal events slightly, and there will be even fewer signal events at higher $T_{b}^{(5 / 3)}$ masses. For these reasons, discovery of even a relatively light $T_{b}^{(5 / 3)}$ would be very difficult at $\sqrt{s}=7 \mathrm{TeV}$, and this discovery potential is not expected to improve substantially at the current LHC centre of mass energy of $\sqrt{s}=8 \mathrm{TeV}$. Therefore, the $T_{b}^{(5 / 3)}$ production cross sections were calculated at the full LHC centre of mass energy of $\sqrt{s}=14 \mathrm{TeV}$, which increases the signal cross section by roughly an order of magnitude.

The jets and leptons are required to be isolated from one another. That is,

$$
\begin{aligned}
& \Delta R_{\ell \ell}=\sqrt{\Delta y_{\ell \ell}^{2}+\Delta \phi_{\ell \ell}^{2}}>0.4 \\
& \Delta R_{\ell j}=\sqrt{\Delta y_{\ell j}^{2}+\Delta \phi_{\ell j}^{2}}>0.4 \\
& \Delta R_{j j}=\sqrt{\Delta y_{j j}^{2}+\Delta \phi_{j j}^{2}}>0.4
\end{aligned}
$$

where $\Delta y_{m n}$ and $\Delta \phi_{m n}$ are the difference in rapidity and azimuthal angle between particles $m$ and $n$. The results were generated at the parton level, with parton showering and hadronization not taken into account. These effects may be included using software such as Pythia [115]. However, they are not needed for the purpose of this study, which is simply to obtain an estimate of the $T_{b}^{(5 / 3)}$ discovery potential 


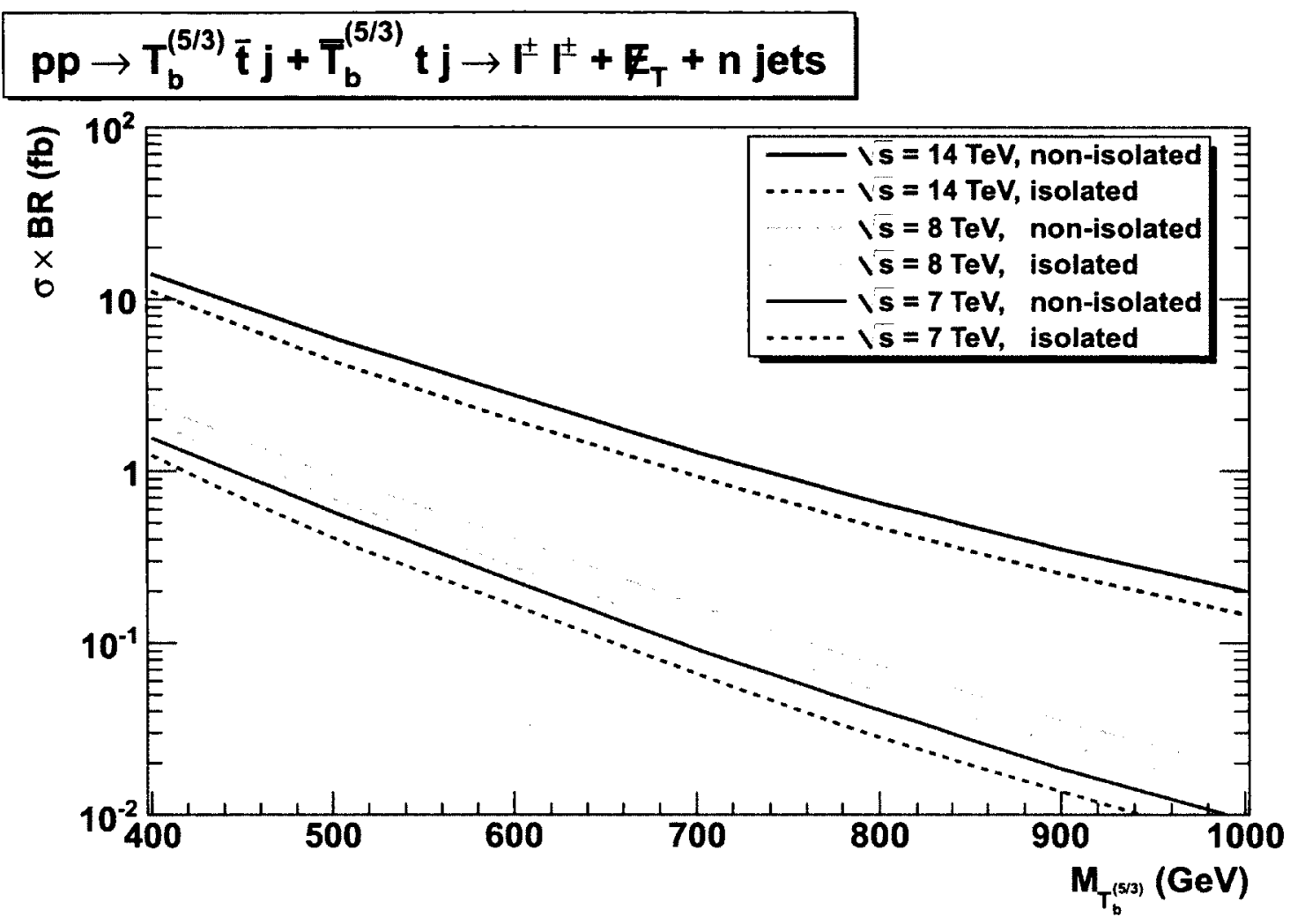

Figure 5.17: Sum of the $T_{b}^{(5 / 3)}$ and $\bar{T}_{b}^{(5 / 3)}$ production cross sections with decays to same-sign dileptons for both the isolated and non-isolated scenarios, at LHC centre of mass energies of $\sqrt{s}=7 \mathrm{TeV}, 8 \mathrm{TeV}$ and $14 \mathrm{TeV}$. Discovery of the $T_{b}^{(5 / 3)}$ heavy quark will be very difficult at $\sqrt{s}=7 \mathrm{TeV}$ and $8 \mathrm{TeV}$ due to the relatively small number of signal events. Therefore, the $T_{b}^{(5 / 3)}$ production cross sections were calculated at the full LHC centre of mass energy of $\sqrt{s}=14 \mathrm{TeV}$, which increases the signal cross section by roughly an order of magnitude. 
Table 5.5: Lepton charge misidentification probability for various ranges of $p_{T}$ [113].

\begin{tabular}{|c|c|c|}
\hline$p_{T}$ & $\epsilon_{\text {charge }}$ (electrons) & $\epsilon_{\text {charge }}$ (muons) \\
\hline $0<p_{T}<100 \mathrm{GeV}$ & $8 \times 10^{-4}$ & $6 \times 10^{-4}$ \\
\hline $100 \mathrm{GeV}<p_{T}<300 \mathrm{GeV}$ & $6 \times 10^{-3}$ & $2 \times 10^{-3}$ \\
\hline $300 \mathrm{GeV}<p_{T}<500 \mathrm{GeV}$ & $2 \times 10^{-2}$ & $1 \times 10^{-2}$ \\
\hline $500 \mathrm{GeV}<p_{T}<1000 \mathrm{GeV}$ & $6 \times 10^{-2}$ & $5 \times 10^{-2}$ \\
\hline$p_{T}>1000 \mathrm{GeV}$ & $2 \times 10^{-1}$ & $2 \times 10^{-1}$ \\
\hline
\end{tabular}

in the Bestest Little Higgs model. The leptons and jets are required to have $p_{T}>10$ $\mathrm{GeV}$. This threshold is necessary for MadGraph to achieve stable results in its cross section calculations, and is also required to reconstruct these particles experimentally.

Previous studies of the charge misidentification backgrounds to heavy quark production have assumed a flat lepton charge misidentification probability [112]. To estimate the lepton charge misidentification probability in this analysis, Figure 10 of [113] was used, which plots the charge misidentification probability as a function of the $p_{T}$ of the misidentified lepton. Depending on the type of lepton (electron or muon) and its $p_{T}$, a charge misidentification probability is assigned on an event-byevent basis, according to Table 5.5. As the large majority of leptons from the charge misidentification backgrounds tend to have $p_{T}<500 \mathrm{GeV}$, the charge misidentification probability is small enough that these backgrounds can be reduced to a manageable level. Nevertheless, the $t \bar{t}$ background is found to be the largest contribution to the total background, even after imposing additional kinematic cuts.

A fake rate of $\epsilon_{\text {fake }}=10^{-4}$ is used for a jet being misidentified as a lepton, which reduces the large $W^{ \pm}+$jets background to a manageable level. The lepton reconstruction efficiency for this fake rate is roughly $90 \%$ for electrons and even higher for muons $[87,113,114]$. However, the fake rate and lepton reconstruction efficiency 
are highly dependent on the jet $p_{T}$ and on the cuts used, so for the purposes of this preliminary analysis, only the jet fake rate of $\epsilon_{f a k e}=10^{-4}$ is included. A more detailed detector simulation would take the effects of lepton reconstruction into account.

The kinematic cuts used in this analysis of $T_{b}^{(5 / 3)}$ production are summarized in Table 5.6. Rapidity cuts on both final state leptons of $|y(\ell)|<2.5$ are imposed to take into account the acceptance of the electromagnetic calorimeter and a hard $p_{T}$ cut is imposed on the hardest lepton only. It is found that a hard $p_{T}$ cut on the hardest lepton implies a certain hardness on the $p_{T}$ of the second lepton. Therefore, imposing an additional hard $p_{T}$ cut on the second lepton, beyond the threshold of $p_{T}>10 \mathrm{GeV}$, is not necessary as this does not help to significantly improve the signal to background ratio. Cuts on the missing transverse energy, $\mathbb{E}_{T}$, and on the invariant mass of the two leptons, $M_{\ell \ell}$, are also imposed, as these cuts completely eliminate the large background from $Z^{*} / \gamma^{*}$ production. The lepton kinematics are found to be highly dependent on the mass of the $T_{b}^{(5 / 3)}$, so the different values of the lepton cuts are listed in Table 5.6 for each value of $M_{T_{b}^{(5 / 3)}}$.

The kinematics of the tagged forward jet, on the other hand, are found to be insensitive to the $T_{b}^{(5 / 3)}$ mass. Therefore, the same cuts on the rapidity, $p_{T}$ and energy of this forward jet are used for each value of $M_{T_{b}^{(5 / 3)}}$. These forward jet tag cuts are also listed in Table 5.6. It should be noted that, although a single energetic forward jet is tagged with high $p_{T}$, all jets are assumed to have transverse momenta greater than the threshold of $p_{T}>10 \mathrm{GeV}$. These choices of cuts are found to be effective in improving the signal to background ratio.

The essence of the same-sign dilepton signal is the decay of a heavy particle, whose energy is distributed among its decay products: leptons, jets and $\not_{T}$. It is therefore 
Table 5.6: Cuts used for $T_{b}^{(5 / 3)}$ single associated production to enhance the signal to background ratio in the same-sign dilepton channel.

\begin{tabular}{|l|l|l|l|}
\hline & $M_{T_{b}^{(5 / 3)}}=400 \mathrm{GeV}$ & $M_{T_{b}^{(5 / 3)}}=600 \mathrm{GeV}$ & $M_{T_{b}^{(5 / 3)}}=800 \mathrm{GeV}$ \\
\hline Leptonic Cuts & $|y(\ell)|<2.5$ & $|y(\ell)|<2.5$ & $|y(\ell)|<2.5$ \\
& $p_{T}\left(\ell_{1}\right)>50 \mathrm{GeV}$ & $p_{T}\left(\ell_{1}\right)>80 \mathrm{GeV}$ & $p_{T}\left(\ell_{1}\right)>150 \mathrm{GeV}$ \\
& $p_{T}\left(\ell_{2}\right)>10 \mathrm{GeV}$ & $p_{T}\left(\ell_{2}\right)>10 \mathrm{GeV}$ & $p_{T}\left(\ell_{2}\right)>10 \mathrm{GeV}$ \\
& $\mathbb{E}_{T}>80 \mathrm{GeV}$ & $\mathbb{E}_{T}>80 \mathrm{GeV}$ & $\mathbb{E}_{T}>80 \mathrm{GeV}$ \\
& $M_{\ell \ell}>120 \mathrm{GeV}$ & $M_{\ell \ell}>160 \mathrm{GeV}$ & $M_{\ell \ell}>240 \mathrm{GeV}$ \\
\hline$H_{T}$ Cut & $H_{T}>450 \mathrm{GeV}$ & $H_{T}>550 \mathrm{GeV}$ & $H_{T}>800 \mathrm{GeV}$ \\
\hline Jet Cuts & \multicolumn{3}{|c|}{$p_{T}(j)>10 \mathrm{GeV}$} \\
& $3.0<\left|y\left(j_{\text {tag }}\right)\right|<5.0$ \\
& \multicolumn{3}{|c}{$p_{T}\left(j_{\text {tag }}\right)>30 \mathrm{GeV}$} \\
& $E\left(j_{\text {tag }}\right)>500 \mathrm{GeV}$ \\
\hline
\end{tabular}

useful to define the total transverse energy, $H_{T}$, as

$$
H_{T}=\sum_{j, \ell, \mathbb{E}_{T}}\left|\vec{p}_{T}\right|
$$

As the $H_{T}$ distribution peaks around the $T_{b}^{(5 / 3)}$ mass, a cut on $H_{T}$ will be very useful in improving the signal to background ratio. This cut is also listed in Table 5.6 for each value of $M_{T_{b}^{(5 / 3)}}$.

\subsubsection{Results}

The LHC cross sections (in $\mathrm{fb}$ ) for the $T_{b}^{(5 / 3)}$ single associated production signals and backgrounds, using the cuts listed in Table 5.6, are tabulated in Tables 5.7-5.9 for $T_{b}^{(5 / 3)}$ masses of $400 \mathrm{GeV}, 600 \mathrm{GeV}$ and $800 \mathrm{GeV}$ for both the isolated and non-isolated 
scenarios. The cross sections in both the $\ell^{+} \ell^{+}$and $\ell^{-} \ell^{-}$channels are shown. As an example, the $H_{T}$ distribution for the sum of these two channels, $\frac{d \sigma}{d H_{T}}\left(\ell^{+} \ell^{+}\right)+\frac{d \sigma}{d H_{T}}\left(\ell^{-} \ell^{-}\right)$, is plotted in Figure 5.18 for the $M_{T_{b}^{(5 / 3)}}=400 \mathrm{GeV}$ signals and backgrounds. As the plots for the $600 \mathrm{GeV}$ and $800 \mathrm{GeV}$ signals are qualitatively similar, they are not shown here.

It is evident from Tables 5.7-5.9 that the signal cross section of the $\ell^{+} \ell^{+}$channel is roughly twice as large as that of the $\ell^{-} \ell^{-}$channel, whereas for the $t \bar{t}$ background these cross sections are identical. Therefore, it is also useful to calculate the difference in the cross sections of these two channels. Although this reduces the number of signal events, it completely eliminates the large contribution from the $t \bar{t}$ background, which improves the discovery potential in the same-sign dilepton channel. The $H_{T}$ distribution for the difference of these two channels, $\frac{d \sigma}{d H_{T}}\left(\ell^{+} \ell^{+}\right)-\frac{d \sigma}{d H_{T}}\left(\ell^{-} \ell^{-}\right)$, is plotted in Figure 5.19 for the $M_{T_{b}^{(5 / 3)}}=400 \mathrm{GeV}$ signals and backgrounds.

The observability of the $T_{b}^{(5 / 3)}$ signal is determined by the relative size of the signal to background, and by the assumed integrated luminosity. The luminosity required to produce a $5 \sigma$ discovery (i.e. $S / \sqrt{B} \geq 5$ ) must therefore be determined. To ensure that an adequate number of signal events are observed, a second criteria is imposed, requiring that there must be at least 10 signal events. Following this procedure, Table 5.10 lists the integrated luminosities required to produce a $5 \sigma$ signal with at least 10 signal events for the three values of $M_{T_{b}^{(5 / 3)}}$ considered, in both the isolated and non-isolated scenarios.

The results of Table 5.10 show that discovery of the $T_{b}^{(5 / 3)}$ heavy quark of a given mass is more promising in the non-isolated scenario, as the larger signal cross section results in less integrated luminosity required for discovery. Furthermore, considering the difference of the $\ell^{+} \ell^{+}$and $\ell^{-} \ell^{-}$channels eliminates the large $t \bar{t}$ background, improving the discovery potential for all three $T_{b}^{(5 / 3)}$ masses considered. This is 
Table 5.7: $\mathrm{LHC}$ cross sections (in $\mathrm{fb}$ ) for $T_{b}^{(5 / 3)}$ single associated production in the same-sign dilepton channel using the cuts listed in Table 5.6 for $M_{T_{b}^{(5 / 3)}}=400 \mathrm{GeV}$ in the isolated and non-isolated scenarios. The cross sections for both the $\ell^{+} \ell^{+}$and $\ell^{-} \ell^{-}$channels are listed. The cross section values obtained using MadGraph5 have statistical uncertainties of $\sim 1 \%$.

\begin{tabular}{|l|ll|l|l|l|}
\hline \multicolumn{6}{|c|}{$M_{T_{b}^{(5 / 3)}}=400 \mathrm{GeV}$} \\
\hline Channel & Process & Leptonic Cuts & + Jet Tag & $+H_{T}$ Cut \\
\hline$\ell^{+} \ell^{+}$ & $W^{+} W^{+}+2,3 \mathrm{j}$ & 2.78 & $1.67 \times 10^{-1}$ & $1.33 \times 10^{-1}$ \\
& $W^{+} W^{+} W^{-}+0,1,2 \mathrm{j}$ & $3.57 \times 10^{-1}$ & $1.72 \times 10^{-2}$ & $1.44 \times 10^{-2}$ \\
& $t \bar{t} W^{+}$ & $+0,1,2 \mathrm{j}$ & 2.30 & $1.56 \times 10^{-1}$ & $1.50 \times 10^{-1}$ \\
& $t \bar{t} W^{+} W^{-}$ & $+0,1 \mathrm{j}$ & $2.44 \times 10^{-2}$ & $3.02 \times 10^{-3}$ & $3.01 \times 10^{-3}$ \\
& $t \bar{t}$ & $+0,1,2 \mathrm{j}$ & 8.01 & $7.96 \times 10^{-1}$ & $6.72 \times 10^{-1}$ \\
& $W^{+} W^{-}$ & $+0,1,2 \mathrm{j}$ & $5.31 \times 10^{-1}$ & $2.59 \times 10^{-2}$ & $1.98 \times 10^{-2}$ \\
& $W^{+}$ & $+1,2,3 \mathrm{j}$ & 6.22 & $4.12 \times 10^{-1}$ & $1.71 \times 10^{-1}$ \\
& $W^{+} Z$ & $+0,1,2 \mathrm{j}$ & 1.70 & $1.34 \times 10^{-1}$ & $5.82 \times 10^{-2}$ \\
& Total Background & $2.19 \times 10^{1}$ & 1.71 & 1.22 \\
& $T_{b}^{(5 / 3)}$ Signal (Iso) & 1.33 & $6.82 \times 10^{-1}$ & $6.76 \times 10^{-1}$ \\
& $T_{b}^{(5 / 3)}$ Signal (Non-Iso) & 1.45 & $7.47 \times 10^{-1}$ & $7.39 \times 10^{-1}$ \\
\hline$\ell^{-} \ell^{-}$ & $W^{-} W^{-}$ & $+2,3 \mathrm{j}$ & $9.65 \times 10^{-1}$ & $4.36 \times 10^{-2}$ & $3.42 \times 10^{-2}$ \\
& $W^{-} W^{-} W^{+}+0,1,2 \mathrm{j}$ & $1.82 \times 10^{-1}$ & $7.73 \times 10^{-3}$ & $6.69 \times 10^{-3}$ \\
& $t \bar{t} W^{-}$ & $+0,1,2 \mathrm{j}$ & 1.07 & $5.49 \times 10^{-2}$ & $5.34 \times 10^{-2}$ \\
& $t \bar{t} W^{+} W^{-} \quad+0,1 \mathrm{j}$ & $2.48 \times 10^{-2}$ & $3.35 \times 10^{-3}$ & $3.35 \times 10^{-3}$ \\
& $t \bar{t}$ & $+0,1,2 \mathrm{j}$ & 7.97 & $7.99 \times 10^{-1}$ & $6.75 \times 10^{-1}$ \\
& $W^{+} W^{-}$ & $+0,1,2 \mathrm{j}$ & $3.82 \times 10^{-1}$ & $1.97 \times 10^{-2}$ & $1.45 \times 10^{-2}$ \\
& $W^{-}$ & $+1,2,3 \mathrm{j}$ & 3.22 & $2.08 \times 10^{-1}$ & $8.40 \times 10^{-2}$ \\
& $W^{-} Z$ & $+0,1,2 \mathrm{j}$ & $9.27 \times 10^{-1}$ & $6.32 \times 10^{-2}$ & $2.83 \times 10^{-2}$ \\
& Total Background & $1.47 \times 10^{1}$ & 1.20 & $8.96 \times 10^{-1}$ \\
& $\bar{T}_{b}^{(5 / 3)}$ Signal (Iso) & $6.73 \times 10^{-1}$ & $2.85 \times 10^{-1}$ & $2.82 \times 10^{-1}$ \\
& $\bar{T}_{b}^{(5 / 3)}$ Signal (Non-Iso) & $7.29 \times 10^{-1}$ & $3.10 \times 10^{-1}$ & $3.07 \times 10^{-1}$ \\
\hline
\end{tabular}


Table 5.8: $\mathrm{LHC}$ cross sections (in $\mathrm{fb}$ ) for $T_{b}^{(5 / 3)}$ single associated production in the same-sign dilepton channel using the cuts listed in Table 5.6 for $M_{T_{b}^{(5 / 3)}}=600 \mathrm{GeV}$ in the isolated and non-isolated scenarios. The cross sections for both the $\ell^{+} \ell^{+}$and $\ell^{-} \ell^{-}$channels are listed. The cross section values obtained using MadGraph5 have statistical uncertainties of $\sim 1 \%$.

\begin{tabular}{|c|c|c|c|c|c|}
\hline \multicolumn{6}{|c|}{$M_{T_{b}^{(5 / 3)}}=600 \mathrm{GeV}$} \\
\hline Channel & \multicolumn{2}{|l|}{ Process } & Leptonic Cuts & + Jet Tag & $+H_{T} \mathrm{Cut}$ \\
\hline \multirow[t]{11}{*}{$\ell^{+} \ell^{+}$} & $W^{+} W^{+}$ & $+2,3 \mathrm{j}$ & 1.60 & $8.81 \times 10^{-2}$ & $6.58 \times 10^{-2}$ \\
\hline & $W^{+} W^{+} W^{-}$ & $+0,1,2 \mathrm{j}$ & $2.19 \times 10^{-1}$ & $1.00 \times 10^{-2}$ & $7.79 \times 10^{-3}$ \\
\hline & $t \bar{t} W^{+}$ & $+0,1,2 \mathrm{j}$ & 1.34 & $8.92 \times 10^{-2}$ & $8.12 \times 10^{-2}$ \\
\hline & $t \bar{t} W^{+} W^{-}$ & $+0,1 \mathrm{j}$ & $1.63 \times 10^{-2}$ & $2.14 \times 10^{-3}$ & $2.14 \times 10^{-3}$ \\
\hline & $t \bar{t}$ & $+0,1,2 \mathrm{j}$ & 5.67 & $5.56 \times 10^{-1}$ & $3.94 \times 10^{-1}$ \\
\hline & $W^{+} W^{-}$ & $+0,1,2 \mathrm{j}$ & $3.97 \times 10^{-1}$ & $1.88 \times 10^{-2}$ & $1.26 \times 10^{-2}$ \\
\hline & $W^{+}$ & $+1,2,3 \mathrm{j}$ & 4.02 & $2.60 \times 10^{-1}$ & $8.34 \times 10^{-2}$ \\
\hline & $W^{+} Z$ & $+0,1,2 \mathrm{j}$ & $8.26 \times 10^{-1}$ & $6.24 \times 10^{-2}$ & $2.11 \times 10^{-2}$ \\
\hline & \multirow{3}{*}{\multicolumn{2}{|c|}{$\begin{array}{l}\text { Total Background } \\
T_{b}^{(5 / 3)} \text { Signal (Iso) } \\
T_{b}^{(5 / 3)} \text { Signal (Non-Iso) }\end{array}$}} & $1.41 \times 10^{1}$ & 1.09 & $6.68 \times 10^{-1}$ \\
\hline & & & $3.89 \times 10^{-1}$ & $2.12 \times 10^{-1}$ & $2.08 \times 10^{-1}$ \\
\hline & & & $5.01 \times 10^{-1}$ & $2.74 \times 10^{-1}$ & $2.68 \times 10^{-1}$ \\
\hline \multirow[t]{11}{*}{$\ell^{-} \ell^{-}$} & $W^{-} W^{-}$ & $+2,3 \mathrm{j}$ & $6.47 \times 10^{-1}$ & $2.65 \times 10^{-2}$ & $1.92 \times 10^{-2}$ \\
\hline & $W^{-} W^{-} W^{+}$ & $+0,1,2 \mathrm{j}$ & $1.16 \times 10^{-1}$ & $4.81 \times 10^{-3}$ & $3.93 \times 10^{-3}$ \\
\hline & $t \tilde{t} W^{-}$ & $+0,1,2 \mathrm{j}$ & $6.54 \times 10^{-1}$ & $3.30 \times 10^{-2}$ & $3.10 \times 10^{-2}$ \\
\hline & $t \bar{t} W^{+} W^{-}$ & $+0,1 \mathrm{j}$ & $1.66 \times 10^{-2}$ & $2.37 \times 10^{-3}$ & $2.37 \times 10^{-3}$ \\
\hline & $t \bar{t}$ & $+0,1,2 \mathrm{j}$ & 5.65 & $5.58 \times 10^{-1}$ & $3.95 \times 10^{-1}$ \\
\hline & $W^{+} W^{-}$ & $+0,1,2 \mathrm{j}$ & $2.83 \times 10^{-1}$ & $1.43 \times 10^{-2}$ & $9.27 \times 10^{-3}$ \\
\hline & $W^{-}$ & $+1,2,3 \mathrm{j}$ & 2.11 & $1.33 \times 10^{-1}$ & $4.20 \times 10^{-2}$ \\
\hline & $W^{-} Z$ & $+0,1,2 \mathrm{j}$ & $4.80 \times 10^{-1}$ & $3.16 \times 10^{-2}$ & $1.12 \times 10^{-2}$ \\
\hline & \multirow{3}{*}{\multicolumn{2}{|c|}{$\begin{array}{l}\text { Total Background } \\
\bar{T}_{b}^{(5 / 3)} \text { Signal (Iso) } \\
\bar{T}_{b}^{(5 / 3)} \text { Signal (Non-Iso) }\end{array}$}} & 9.96 & $8.04 \times 10^{-1}$ & $5.14 \times 10^{-1}$ \\
\hline & & & $1.88 \times 10^{-1}$ & $8.59 \times 10^{-2}$ & $8.39 \times 10^{-2}$ \\
\hline & & & $2.41 \times 10^{-1}$ & $1.10 \times 10^{-1}$ & $1.08 \times 10^{-1}$ \\
\hline
\end{tabular}


Table 5.9: LHC cross sections (in $\mathrm{fb}$ ) for $T_{b}^{(5 / 3)}$ single associated production in the same-sign dilepton channel using the cuts listed in Table 5.6 for $M_{T_{b}^{(5 / 3)}}=800 \mathrm{GeV}$ in the isolated and non-isolated scenarios. The cross sections for both the $\ell^{+} \ell^{+}$and $\ell^{-} \ell^{-}$channels are listed. The cross section values obtained using MadGraph5 have statistical uncertainties of $\sim 1 \%$.

\begin{tabular}{|c|c|c|c|c|c|}
\hline \multicolumn{6}{|c|}{$M_{T_{b}^{(5 / 3)}}=800 \mathrm{GeV}$} \\
\hline Channel & \multicolumn{2}{|l|}{ Process } & Leptonic Cuts & + Jet Tag & $+H_{T} \mathrm{Cut}$ \\
\hline \multirow[t]{11}{*}{$\ell^{+} \ell^{+}$} & $W^{+} W^{+}$ & $+2,3 \mathrm{j}$ & $5.11 \times 10^{-1}$ & $2.35 \times 10^{-2}$ & $1.47 \times 10^{-2}$ \\
\hline & $W^{+} W^{+} W^{-}$ & $+0,1,2 \mathrm{j}$ & $8.16 \times 10^{-2}$ & $3.33 \times 10^{-3}$ & $2.07 \times 10^{-3}$ \\
\hline & $t \bar{t} W^{+}$ & $+0,1,2 \mathrm{j}$ & $4.66 \times 10^{-1}$ & $2.66 \times 10^{-2}$ & $2.03 \times 10^{-2}$ \\
\hline & $t \bar{t} W^{+} W^{-}$ & $+0,1 \mathrm{j}$ & $6.67 \times 10^{-3}$ & $1.04 \times 10^{-3}$ & $1.02 \times 10^{-3}$ \\
\hline & $t \bar{t}$ & $+0,1,2 \mathrm{j}$ & 2.15 & $1.95 \times 10^{-1}$ & $1.00 \times 10^{-1}$ \\
\hline & $W^{+} W^{-}$ & $+0,1,2 \mathrm{j}$ & $2.04 \times 10^{-1}$ & $9.34 \times 10^{-3}$ & $5.70 \times 10^{-3}$ \\
\hline & $W^{+}$ & $+1,2,3 \mathrm{j}$ & 1.63 & $9.93 \times 10^{-2}$ & $1.88 \times 10^{-2}$ \\
\hline & $W^{+} Z$ & $+0,1,2 \mathrm{j}$ & $2.04 \times 10^{-1}$ & $1.40 \times 10^{-2}$ & $1.98 \times 10^{-3}$ \\
\hline & \multirow{3}{*}{\multicolumn{2}{|c|}{$\begin{array}{l}\text { Total Background } \\
T_{b}^{(5 / 3)} \text { Signal (Iso) } \\
T_{b}^{(5 / 3)} \text { Signal (Non-Iso) }\end{array}$}} & 5.25 & $3.72 \times 10^{-1}$ & $1.65 \times 10^{-1}$ \\
\hline & & & $8.14 \times 10^{-2}$ & $4.62 \times 10^{-2}$ & $3.98 \times 10^{-2}$ \\
\hline & & & $1.07 \times 10^{-1}$ & $6.07 \times 10^{-2}$ & $5.22 \times 10^{-2}$ \\
\hline \multirow[t]{11}{*}{$\ell^{-} \ell^{-}$} & $W^{-} W^{-}$ & $+2,3 \mathrm{j}$ & $2.67 \times 10^{-1}$ & $9.32 \times 10^{-3}$ & $5.34 \times 10^{-3}$ \\
\hline & $W^{-} W^{-} W^{+}$ & $+0,1,2 \mathrm{j}$ & $4.63 \times 10^{-2}$ & $1.86 \times 10^{-3}$ & $1.25 \times 10^{-3}$ \\
\hline & $t \bar{t} W^{-}$ & $+0,1,2 \mathrm{j}$ & $2.28 \times 10^{-1}$ & $1.13 \times 10^{-2}$ & $9.55 \times 10^{-3}$ \\
\hline & $t \bar{t} W^{+} W^{-}$ & $+0,1 \mathrm{j}$ & $6.90 \times 10^{-3}$ & $1.14 \times 10^{-3}$ & $1.11 \times 10^{-3}$ \\
\hline & $t \bar{t}$ & $+0,1,2 \mathrm{j}$ & 2.14 & $1.95 \times 10^{-1}$ & $1.00 \times 10^{-1}$ \\
\hline & $W^{+} W^{-}$ & $+0,1,2 \mathrm{j}$ & $1.43 \times 10^{-1}$ & $6.88 \times 10^{-3}$ & $3.63 \times 10^{-3}$ \\
\hline & $W^{-}$ & $+1,2,3 j$ & $8.68 \times 10^{-1}$ & $5.35 \times 10^{-2}$ & $9.45 \times 10^{-3}$ \\
\hline & $W^{-Z}$ & $+0,1,2 \mathrm{j}$ & $1.30 \times 10^{-1}$ & $8.32 \times 10^{-3}$ & $1.79 \times 10^{-3}$ \\
\hline & \multirow{3}{*}{\multicolumn{2}{|c|}{$\begin{array}{l}\text { Total Background } \\
\bar{T}_{b}^{(5 / 3)} \text { Signal (Iso) } \\
\bar{T}_{b}^{(5 / 3)} \text { Signal (Non-Iso) }\end{array}$}} & 3.84 & $2.87 \times 10^{-1}$ & $1.33 \times 10^{-1}$ \\
\hline & & & $3.77 \times 10^{-2}$ & $1.82 \times 10^{-2}$ & $1.54 \times 10^{-2}$ \\
\hline & & & $4.89 \times 10^{-2}$ & $2.38 \times 10^{-2}$ & $2.03 \times 10^{-2}$ \\
\hline
\end{tabular}




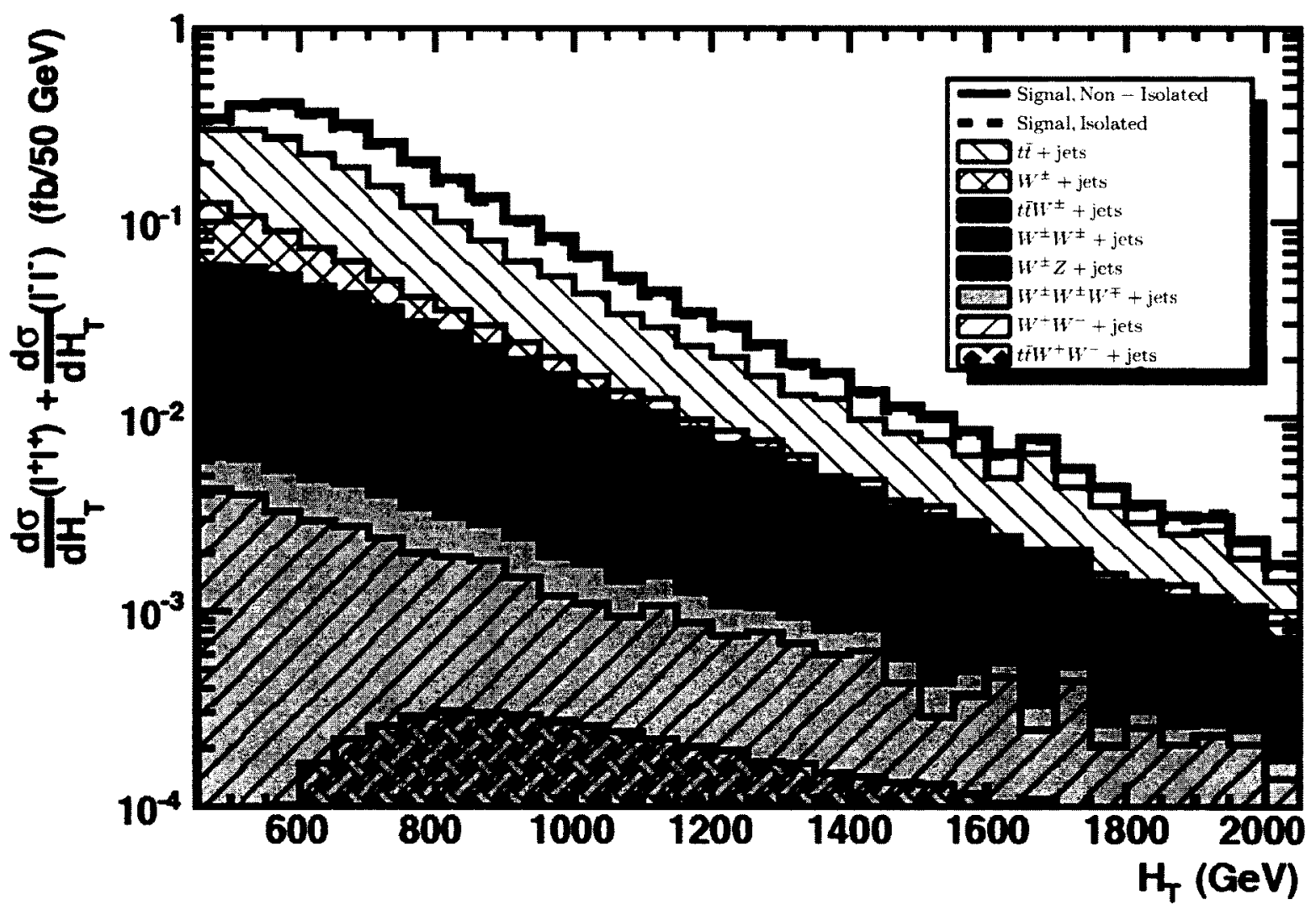

Figure 5.18: $H_{T}$ distributions for $T_{b}^{(5 / 3)}$ production signals and backgrounds, based on the cross sections of Table 5.7 for $M_{T_{b}^{(5 / 3)}}=400 \mathrm{GeV}$ in the isolated and nonisolated scenarios. This plot shows the sum, $\frac{d \sigma}{d H_{T}}\left(\ell^{+} \ell^{+}\right)+\frac{d \sigma}{d H_{T}}\left(\ell^{-} \ell^{-}\right)$, of the two same-sign dilepton channels. The backgrounds are stacked, and the two signals are shown as a broad enhancement above the background. 


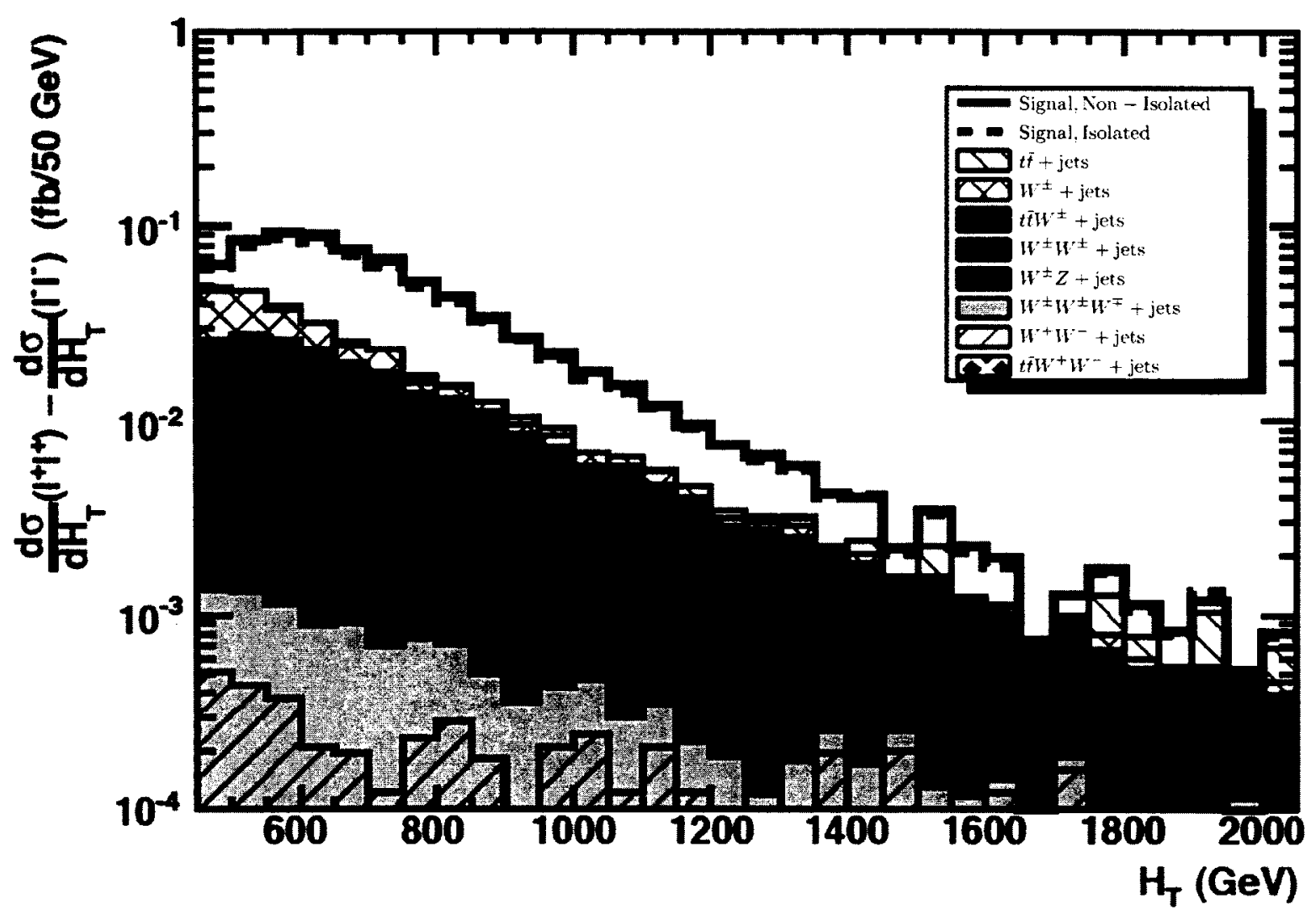

Figure 5.19: $H_{T}$ distributions for $T_{b}^{(5 / 3)}$ production signals and backgrounds, based on the cross sections of Table 5.7 for $M_{T_{b}^{(5 / 3)}}=400 \mathrm{GeV}$ in the isolated and nonisolated scenarios. This plot shows the difference, $\frac{d \sigma}{d H_{T}}\left(\ell^{+} \ell^{+}\right)-\frac{d \sigma}{d H_{T}}\left(\ell^{-} \ell^{-}\right)$, of the two same-sign dilepton channels. The backgrounds are stacked, and the two signals are shown as a broad enhancement above the background. 
Table 5.10: Discovery potential of the $T_{b}^{(5 / 3)}$ heavy quark for both the sum and difference of the $\ell^{+} \ell^{+}$and $\ell^{-} \ell^{-}$channels. The signal and background cross sections (in $\mathrm{fb}$ ), calculated from the values in Tables 5.7-5.9, are listed for each scenario considered. Also listed are the integrated luminosities (in $\mathrm{fb}^{-1}$ ) required for a statistical significance of $S / \sqrt{B} \geq 5$ and at least 10 signal events in each scenario, after the cuts of Table 5.6 have been imposed.

\begin{tabular}{|l|c|l|l|l|}
\hline \multicolumn{5}{|c|}{ Sum of $\ell^{+} \ell^{+}$and $\ell^{-} \ell^{-}$Channels } \\
\hline Scenario & $M_{T_{b}^{(5 / 3)}}(\mathrm{GeV})$ & $\sigma_{S}(\mathrm{fb})$ & $\sigma_{B}(\mathrm{fb})$ & $\mathcal{L}\left(5 \sigma \& N_{S}=10\right)\left(\mathrm{fb}^{-1}\right)$ \\
\hline Isolated & 400 & 0.958 & 2.12 & 58 \\
& 600 & 0.292 & 1.18 & 347 \\
& 800 & 0.0552 & 0.298 & 2440 \\
\hline Non-Isolated & 400 & 1.05 & 2.12 & 48 \\
& 600 & 0.376 & 1.18 & 210 \\
& 800 & 0.0725 & 0.298 & 1420 \\
\hline \multirow{5}{*}{ Scenario } & $M_{T_{b}^{(5 / 3)}}(\mathrm{GeV})$ & $\sigma_{S}(\mathrm{fb})$ & $\sigma_{B}(\mathrm{fb})$ & $\mathcal{L}\left(5 \sigma \& N_{S}=10\right)\left(\mathrm{fb}^{-1}\right)$ \\
\hline Isolated & 400 & 0.394 & 0.324 & 52 \\
& 600 & 0.124 & 0.154 & 250 \\
& 800 & 0.0243 & 0.0325 & 1380 \\
\hline Non-Isolated & 400 & 0.432 & 0.324 & 43 \\
& 600 & 0.161 & 0.154 & 149 \\
& 800 & 0.0319 & 0.0325 & 797 \\
\hline
\end{tabular}


especially useful for improving the signal to background ratio at higher $T_{b}^{(5 / 3)}$ masses, at which the relatively low number of signal events are difficult to observe if the large $t \bar{t}$ background is present.

The $T_{b}^{(5 / 3)}$ heavy quark with masses of 400,600 and $800 \mathrm{GeV}$ can be discovered at the LHC with integrated luminosities as low as 52,250 and $1380 \mathrm{fb}^{-1}$, respectively, in the isolated scenario, and as low as 43,149 and $797 \mathrm{fb}^{-1}$, respectively, in the non-isolated scenario. Although a $T_{b}^{(5 / 3)}$ with a mass of $400 \mathrm{GeV}$ is excluded in the non-isolated scenario, as it is slightly lighter than the $419 \mathrm{GeV}$ discovery limit presented in Equation 5.42 , a $400 \mathrm{GeV} \mathrm{T}_{b}^{(5 / 3)}$ is not excluded in the isolated scenario, according to Equation 5.41 .

The early years of LHC running are expected to produce $\sim 10 \mathrm{fb}^{-1} /$ year. It is therefore unlikely that a $T_{b}^{(5 / 3)}$ state heavier than about $400 \mathrm{GeV}$ would be observed at the LHC in this period of time. Once design luminosity is achieved, it is expected that $\sim 100 \mathrm{fb}^{-1}$ will be produced per year. Therefore, discovering the $T_{b}^{(5 / 3)}$ heavy quark of the Bestest Little Higgs model with a mass between $400 \mathrm{GeV}$ and $600 \mathrm{GeV}$ is possible at the LHC, although the amount of integrated luminosity required depends strongly on the values of the parameters $\tan \theta_{12}$ and $\tan \theta_{13}$. Discovering a $T_{b}^{(5 / 3)}$ heavy quark with a mass of $800 \mathrm{GeV}$ or higher will be very difficult at the LHC, particularly in the isolated scenario. However, in the Bestest Little Higgs model, $T_{b}^{(5 / 3)}$ masses heavier than this tend to be excluded by the upper limits of Equations 5.41-5.42 from fine-tuning constraints.

If the $T_{b}^{(5 / 3)}$ heavy quark of the Bestest Little Higgs model were observed at the LHC via the single associated production process, measuring the production cross section would allow a determination of the value of $\tan \theta_{13}$, as the $W^{+} \bar{T}_{b}^{(5 / 3)} t$ vertex 
appearing in Figure 5.16 is given by

$$
-i \sqrt{2} g \cos \theta_{13} \frac{v_{1}}{f} \gamma_{\mu} P_{R}+\mathcal{O}\left(\frac{v^{2}}{f^{2}}\right)
$$

However, the $T_{b}^{(5 / 3)}$ heavy quark cannot be fully reconstructed in the same-sign dilepton channel due to the large missing energy involved in its leptonic decay. Any excess of events in the same-sign dilepton channel would not identify whether the $T_{b}^{(5 / 3)}$ of the Bestest Little Higgs model is responsible for this excess. Considering hadronic decays allows the $T_{b}^{(5 / 3)}$ to be fully reconstructed, but this channel involves a large number of jets in the final state, and therefore, large QCD backgrounds. Much of this analysis would depend on the efficiency for reconstructing the $T_{b}^{(5 / 3)}$ in its hadronic decay modes while simultaneously reducing these backgrounds. Kaplan et al. proposed a method for identifying boosted hadronically decaying top quarks, with an efficiency of about $40 \%$ [116]. As the top quark from the $T_{b}^{(5 / 3)}$ decay tends to have $p_{T}>500 \mathrm{GeV}$ in this study, applying their method to reconstruct a hadronically decaying $T_{b}^{(5 / 3)}$ may be worth considering and is left for a future study.

\subsection{Summary}

This chapter presented a preliminary exploration of the heavy quark phenomenology in the Bestest Little Higgs model at the LHC. In addition to exploring the regions of parameter space allowed by fine-tuning constraints, the lower mass limits of the lightest top partners were determined using recent CMS results for two scenarios of the parameters $\tan \theta_{12}$ and $\tan \theta_{13}$. Lower mass limits of $392 \mathrm{GeV}$ for the isolated scenario and $419 \mathrm{GeV}$ for the non-isolated scenario were determined. The LO and NLO cross sections for single top partner production were then calculated and it was determined that the single production cross sections are higher than that of 
pair production, particularly at heavy quark masses above approximately $500 \mathrm{GeV}$. Finally, the associated single production process of the exotic charge $5 / 3$ heavy quark, denoted by $T_{b}^{(5 / 3)}$, was studied in greater detail. It was found that the $T_{b}^{(5 / 3)}$ heavy quark with masses of 400,600 and $800 \mathrm{GeV}$ could be discovered in this channel at the LHC with integrated luminosities as low as 52,250 and $1380 \mathrm{fb}^{-1}$, respectively, in the isolated scenario, and as low as 43,149 and $797 \mathrm{fb}^{-1}$, respectively, in the non-isolated scenario. These heavy quark production channels are worthwhile for the LHC experiments to explore further. 


\section{Chapter 6}

\section{Conclusions}

Little Higgs models provide an elegant solution to the hierarchy problem of the Standard Model, applying collective symmetry breaking to ensure that a quadratically divergent Higgs mass is not generated at one loop, without the need for introducing fine-tuning. This collective symmetry breaking is implemented in the gauge, fermion and Higgs sectors, implying the existence of partner particles at the $\mathrm{TeV}$ scale for most of the Standard Model particles. The research carried out in this thesis focused on the LHC phenomenology of two such Little Higgs models: the Littlest Higgs model and the Bestest Little Higgs model.

Following an overview of the Standard Model in Chapter 2, Chapter 3 presented the results of a study of Higgs triplet boson production in the Littlest Higgs model in the $W^{ \pm} W^{ \pm}, W^{ \pm} Z, W^{+} W^{-}$, and $Z Z$ channels at the LHC for a centre of mass energy of $\sqrt{s}=14 \mathrm{TeV}$, comparing these results with the predictions of two additional Higgs

triplet models: the Georgi-Machacek model and the Left-Right Symmetric model. The Higgs triplet production cross sections were found to be highly sensitive to the triplet vacuum expectation value (vev), $v^{\prime}$, which is already tightly constrained by precision electroweak data.

Observing a Higgs triplet at the LHC is most promising in the Georgi-Machacek 
model due to a weaker constraint on the triplet vev. In this model, a Higgs triplet boson with a mass of 1.0 (1.5) $\mathrm{TeV}$ can be observed at the LHC with an integrated luminosity as low as $41(119) \mathrm{fb}^{-1}$ in the $W^{ \pm} W^{ \pm}$channel and as low as $171(474) \mathrm{fb}^{-1}$ in the $W^{ \pm} Z$ channel, for $v^{\prime}=39 \mathrm{GeV}$. The $W^{ \pm} W^{ \pm}$channel is the most promising discovery channel due to its distinctive final state. However, it is still possible to observe a signal in the $W^{ \pm} Z$ channel for certain regions of parameter space. The observation of a signal in this channel is a smoking gun for a Higgs triplet, as this process does not occur in Two Higgs Doublet Models. Discovery of the neutral Higgs triplet state is least promising due to the large QCD and t-quark backgrounds in the $W^{+} W^{-}$channel and the small signal cross section in the $Z Z \rightarrow \ell^{+} \ell^{-} \ell^{+} \ell^{-}$channel. Including the $Z Z \rightarrow \ell^{+} \ell^{-} \nu \bar{\nu}$ channel may slightly improve the $\Phi^{0}$ discovery potential. Considering semileptonic decay modes, which have higher branching ratios than the fully leptonic decay modes, could also potentially improve the Higgs triplet discovery potential. An analysis of these decay channels is left for a future study. Nevertheless, a significant amount of integrated luminosity is required for some of the channels and models, which will require the capabilities of the proposed luminosity upgrades to the LHC, known as the Super LHC (SLHC).

Although Little Higgs models offer an attractive solution to the hierarchy problem, they generally suffer from three major problems. Firstly, it is difficult to generate a Higgs quartic coupling that is required to generate the masses of the Standard Model particles. Secondly, the scalar sector must not include any dangerous singlets, which introduce quadratically divergent Higgs mass and singlet tadpole terms after being integrated out of the theory. Thirdly, Little Higgs models are highly constrained by precision electroweak data, implying that the gauge boson partners must be relatively heavy, while avoiding fine-tuning in the top sector requires that the top partners are relatively light. However, in most Little Higgs models, the top partners are 
heavier than the gauge boson partners, leading to significant fine-tuning in the Higgs potential.

The Bestest Little Higgs model was constructed to solve all three of these problems. A Higgs quartic coupling is generated without introducing any dangerous singlets to the scalar sector. Meanwhile, relatively heavy gauge boson partners allow the model to evade precision electroweak constraints, and fine-tuning is avoided in the top sector because the top partners are relatively light. In Chapter 4, the structure of the Bestest Little Higgs model was described in detail, including the procedure for determining the particle mass eigenstates and deriving the Feynman rules of this model.

As the Bestest Little Higgs model predicts the existence of several relatively light top partners, Chapter 5 presented an analysis of the heavy quark phenomenology in the Bestest Little Higgs model at the LHC. In addition to exploring the regions of parameter space allowed by fine-tuning constraints, the lower mass limits of the lightest top partners were determined using recent CMS results for two scenarios of the heavy quark mixing parameters, $\tan \theta_{12}$ and $\tan \theta_{13}$. Lower mass limits of $392 \mathrm{GeV}$ for the isolated scenario and $419 \mathrm{GeV}$ for the non-isolated scenario were determined. The LO and NLO cross sections for single top partner production were then calculated and it was determined that the single production cross sections are higher than that of pair production, particularly at heavy quark masses above approximately $500 \mathrm{GeV}$, as expected. Finally, associated single production of the exotic charge $5 / 3$ heavy quark, denoted by $T_{b}^{(5 / 3)}$, was studied in detail. Applying stringent kinematic cuts to reduce backgrounds, it was found that a $T_{b}^{(5 / 3)}$ heavy quark with a mass of $400 \mathrm{GeV}, 600$ $\mathrm{GeV}$, or $800 \mathrm{GeV}$ could be discovered in this channel at the LHC with an integrated luminosity as low as 52,250 , and $1380 \mathrm{fb}^{-1}$, respectively, in the isolated scenario, and as low as 43,149 , and $797 \mathrm{fb}^{-1}$, respectively, in the non-isolated scenario. Applying 
the top-tagging method of Kaplan et al. [116], it may be possible to fully reconstruct the $T_{b}^{(5 / 3)}$ heavy quark in its hadronic decay modes, and if it were discovered at the LHC, a determination of the parameter $\tan \theta_{13}$ could be made. These heavy quark production channels are worthwhile for the LHC experiments to explore further. 


\section{Appendix A}

\section{Notations and Conventions}

Using $\hbar=c=1$ units, the four-momentum of a particle may be written as

$$
p_{\mu}=\left(p_{0}, p_{1}, p_{2}, p_{3}\right)=(E, \vec{p})
$$

where $E$ and $\vec{p}=\left(p_{1}, p_{2}, p_{3}\right)=\left(p_{x}, p_{y}, p_{z}\right)$ are the particle's energy and threemomentum, respectively.

The $4 \times 4$ Dirac matrices, $\gamma^{\mu}$, satisfy the relation

$$
\left\{\gamma^{\mu}, \gamma^{\nu}\right\}=\gamma^{\mu} \gamma^{\nu}+\gamma^{\nu} \gamma^{\mu}=2 g^{\mu \nu}
$$

where $g^{\mu \nu}$ is the Minkowski metric defined by

$$
g^{\mu \nu}=\operatorname{diag}(1,-1,-1,-1)
$$

It is useful to consider a particular representation of the $\gamma$ matrices, given by [95]:

$$
\gamma^{\mu}=\left(\begin{array}{cc}
0 & \tau^{\mu} \\
\bar{\tau}^{\mu} & 0
\end{array}\right)
$$


where

$$
\tau^{0}=\bar{\tau}^{0}=\mathbb{1}_{2}=\left(\begin{array}{cc}
1 & 0 \\
0 & 1
\end{array}\right)
$$

is the $2 \times 2$ unit matrix, and $\tau^{i}(i=1,2,3)$ are the Pauli matrices given by

$$
\tau^{1}=-\bar{\tau}^{1}=\left(\begin{array}{cc}
0 & 1 \\
1 & 0
\end{array}\right), \quad \tau^{2}=-\bar{\tau}^{2}=\left(\begin{array}{cc}
0 & -i \\
i & 0
\end{array}\right), \quad \tau^{3}=-\bar{\tau}^{3}=\left(\begin{array}{cc}
1 & 0 \\
0 & -1
\end{array}\right)
$$

Another important matrix, $\gamma_{5}$, is given by [95]

$$
\gamma_{5}=\gamma^{5}=i \gamma^{0} \gamma^{1} \gamma^{2} \gamma^{3}=\left(\begin{array}{cc}
-\mathbb{1}_{2} & 0 \\
0 & \mathbb{1}_{2}
\end{array}\right)
$$

as the left- and right-handed chiral projections of fermions can be written as

$$
\begin{aligned}
& \psi_{L}=P_{L} \psi=\frac{1}{2}\left(1-\gamma_{5}\right) \psi \\
& \psi_{R}=P_{R} \psi=\frac{1}{2}\left(1+\gamma_{5}\right) \psi
\end{aligned}
$$




\section{Appendix B}

\section{The Bestest Little Higgs Model}

\section{B.1 Group Theory Results}

An $S O(4)$ group can be written as a product of $S U(2)_{L} \times S U(2)_{R}$ groups. In terms of the $S O(4)$ generators, which are imaginary anti-symmetric matrices, the $S U(2)$ generators are given by $[21]$

$$
T_{L}^{1}=\frac{i}{2}\left(\begin{array}{cccc}
0 & 0 & 0 & 1 \\
0 & 0 & 1 & 0 \\
0 & -1 & 0 & 0 \\
-1 & 0 & 0 & 0
\end{array}\right), \quad T_{R}^{1}=\frac{i}{2}\left(\begin{array}{cccc}
0 & 0 & 0 & -1 \\
0 & 0 & 1 & 0 \\
0 & -1 & 0 & 0 \\
1 & 0 & 0 & 0
\end{array}\right),
$$


$T_{L}^{2}=\frac{i}{2}\left(\begin{array}{cccc}0 & 0 & 1 & 0 \\ 0 & 0 & 0 & -1 \\ -1 & 0 & 0 & 0 \\ 0 & 1 & 0 & 0\end{array}\right), \quad T_{R}^{2}=\frac{i}{2}\left(\begin{array}{cccc}0 & 0 & -1 & 0 \\ 0 & 0 & 0 & -1 \\ 1 & 0 & 0 & 0 \\ 0 & 1 & 0 & 0\end{array}\right)$

$$
T_{L}^{3}=\frac{i}{2}\left(\begin{array}{cccc}
0 & 1 & 0 & 0 \\
-1 & 0 & 0 & 0 \\
0 & 0 & 0 & 1 \\
0 & 0 & -1 & 0
\end{array}\right), \quad T_{R}^{3}=\frac{i}{2}\left(\begin{array}{cccc}
0 & -1 & 0 & 0 \\
1 & 0 & 0 & 0 \\
0 & 0 & 0 & 1 \\
0 & 0 & -1 & 0
\end{array}\right) \text {. }
$$

The $S O(6)$ generators needed for the calculations performed in this thesis are $6 \times 6$ matrices given by [21]

$$
T^{a}=\left(\begin{array}{cc}
T_{L}^{a} & 0_{2 \times 2} \\
0_{2 \times 2} & 0_{2 \times 2}
\end{array}\right), \quad T^{\prime a}=\left(\begin{array}{cc}
T_{R}^{a} & 0_{2 \times 2} \\
0_{2 \times 2} & 0_{2 \times 2}
\end{array}\right) \quad(a=1,2,3)
$$

\section{B.2 Scalar Mass Eigenstates}

Shifting the Higgs fields by their vevs, $h_{i 1} \rightarrow h_{i 1}+v_{i}(i=1,2)$, in the Higgs potential of Equation 4.27 and using the relations of Equations 4.32-4.35 results in the following 
Higgs doublet mass terms:

$$
\begin{aligned}
V_{\text {Higgs mass }}= & \frac{1}{2}\left(h_{11}, h_{21}\right)\left(\begin{array}{cc}
\frac{B_{\mu}}{\tan \beta} & -\left(B_{\mu}-\lambda_{0} v^{2} \sin 2 \beta\right) \\
-\left(B_{\mu}-\lambda_{0} v^{2} \sin 2 \beta\right) & B_{\mu} \tan \beta
\end{array}\right)\left(\begin{array}{c}
h_{11} \\
h_{21}
\end{array}\right) \\
& +\frac{1}{2} \sum_{j=2}^{4}\left(h_{1 j}, h_{2 j}\right)\left(\begin{array}{cc}
m_{1}^{2} & -m_{1} m_{2} \\
-m_{1} m_{2} & m_{2}^{2}
\end{array}\right)\left(\begin{array}{c}
h_{1 j} \\
h_{2 j}
\end{array}\right) .
\end{aligned}
$$

Diagonalizing the 3 identical mass matrices in the unbroken directions, shown on the second line of Equation B.5, is relatively straightforward. The resulting mass eigenstates are

$$
\begin{aligned}
& \left(\begin{array}{l}
G^{0} \\
A^{0}
\end{array}\right)=\left(\begin{array}{cc}
\sin \beta & \cos \beta \\
-\cos \beta & \sin \beta
\end{array}\right)\left(\begin{array}{l}
h_{12} \\
h_{22}
\end{array}\right) \\
& \left(\begin{array}{l}
G^{ \pm} \\
H^{ \pm}
\end{array}\right)=\left(\begin{array}{ll}
\sin \beta & \cos \beta \\
-\cos \beta & \sin \beta
\end{array}\right)\left(\begin{array}{l}
h_{1}^{ \pm} \\
h_{2}^{ \pm}
\end{array}\right)
\end{aligned}
$$

where

$$
h_{i}^{ \pm} \equiv \frac{1}{\sqrt{2}}\left(h_{i 3} \pm i h_{i 4}\right), \quad(i=1,2)
$$

and

$$
\sin \beta=\frac{m_{2}}{\sqrt{m_{1}^{2}+m_{2}^{2}}} \quad \cos \beta=\frac{m_{1}}{\sqrt{m_{1}^{2}+m_{2}^{2}}} .
$$


Diagonalizing the remaining mass matrix, shown on the first line of Equation B.5, is more complicated and yields the following mass eigenstates

$$
\left(\begin{array}{c}
h^{0} \\
H^{0}
\end{array}\right)=\left(\begin{array}{cc}
\cos \alpha & \sin \alpha \\
-\sin \alpha & \cos \alpha
\end{array}\right)\left(\begin{array}{l}
h_{11} \\
h_{21}
\end{array}\right) \approx\left(\begin{array}{cc}
\sin \beta & \cos \beta \\
-\cos \beta & \sin \beta
\end{array}\right)\left(\begin{array}{l}
h_{11} \\
h_{21}
\end{array}\right)
$$

where

$\tan \alpha=\frac{1}{B_{\mu}-\lambda_{0} v^{2} \sin 2 \beta}\left(B_{\mu} \cot 2 \beta+\sqrt{\frac{B_{\mu}^{2}}{\sin ^{2} 2 \beta}-2 \lambda_{0} B_{\mu} v^{2} \sin 2 \beta+\lambda_{0}^{2} v^{4} \sin ^{2} 2 \beta}\right)$.

The masses of these physical Higgs doublet states are given by Equations 4.37-4.39.

The mass eigenstates for the scalar triplets are also needed. One linear combination of the triplets $\phi^{a}$ and $\chi^{a}$ are eaten to give the heavy gauge bosons their masses, while the orthogonal combination remains as the physical triplet [21]:

$$
\begin{aligned}
& \phi_{\text {eaten }}^{a}=\frac{1}{\sqrt{f^{2}+F^{2}}}\left(f \phi^{a}+F \chi^{a}\right) \quad(a=1,2,3) \\
& \phi_{\text {phys }}^{a}=\frac{1}{\sqrt{f^{2}+F^{2}}}\left(F \phi^{a}-f \chi^{a}\right) \quad(a=1,2,3) \text {. }
\end{aligned}
$$

The unitary gauge will be used, in which the eaten triplet does not appear. The triplet mass eigenstates are given by

$$
\begin{aligned}
\phi^{ \pm} & =\frac{1}{\sqrt{2}}\left(\phi_{p h y s}^{1} \mp i \phi_{p h y s}^{2}\right) \\
\phi^{0} & =\phi_{p h y s}^{3} .
\end{aligned}
$$


The mass eigenstates for the $\eta$ triplet are given by

$$
\begin{aligned}
\eta^{ \pm} & =\frac{1}{\sqrt{2}}\left(\eta^{1} \mp i \eta^{2}\right) \\
\eta^{0} & =\eta^{3}
\end{aligned}
$$

\section{B.3 Gauge Boson Mass Eigenstates}

There are seven gauge bosons, $\left\{A_{1}^{a}, A_{2}^{a}, B^{3}\right\}(a=1,2,3)$, with gauge couplings $\left\{g_{A}, g_{B}, g^{\prime}\right\}$, respectively. It should be noted that, in the following, the Lorentz indices have been suppressed for simplicity. The $7 \times 7$ gauge boson mass matrix can be split up into a $3 \times 3$ neutral gauge boson mass matrix in the $\left\{A_{1}^{3}, A_{2}^{3}, B^{3}\right\}$ basis and two identical $2 \times 2$ charged gauge boson mass matrices in the $\left\{A_{1}^{j}, A_{2}^{j}\right\}$ basis $(j=1,2)$, since these two sectors do not mix with each other. The gauge boson mass terms may be written as

$$
\mathcal{L}_{\text {gauge mass }}=\frac{1}{2}\left(A_{1}^{3}, A_{2}^{3}, B^{3}\right) M_{n}^{2}\left(\begin{array}{c}
A_{1}^{3} \\
A_{2}^{3} \\
B^{3}
\end{array}\right)+\frac{1}{2} \sum_{j=1}^{2}\left(A_{1}^{j}, A_{2}^{j}\right) M_{c}^{2}\left(\begin{array}{c}
A_{1}^{j} \\
A_{2}^{j}
\end{array}\right)
$$


where, expanding as a power series up to $\mathcal{O}\left(\frac{v^{4}}{f^{2}}\right)$, the neutral gauge boson mass matrix after EWSB is given by

$$
\begin{aligned}
M_{n}^{2}= & \frac{1}{4}\left(\begin{array}{ccc}
g_{A}^{2} & -g_{A} g_{B} & 0 \\
-g_{A} g_{B} & g_{B}^{2} & 0 \\
0 & 0 & 0
\end{array}\right)\left(f^{2}+F^{2}\right) \\
& +\frac{1}{8}\left(\begin{array}{ccc}
0 & g_{A} g_{B} & -g_{A} g^{\prime} \\
g_{A} g_{B} & 0 & -g_{B} g^{\prime} \\
-g_{A} g^{\prime} & -g_{B} g^{\prime} & 2 g^{\prime 2}
\end{array}\right)\left(v^{2}-\frac{1}{6} \frac{v^{4}}{f^{2}}\right)
\end{aligned}
$$

and the charged gauge boson mass matrix after EWSB is given by

$$
M_{c}^{2}=\frac{1}{4}\left(\begin{array}{cc}
g_{A}^{2} & -g_{A} g_{B} \\
-g_{A} g_{B} & g_{B}^{2}
\end{array}\right)\left(f^{2}+F^{2}\right)+\frac{1}{8} g_{A} g_{B}\left(\begin{array}{cc}
0 & 1 \\
1 & 0
\end{array}\right)\left(v^{2}-\frac{1}{6} \frac{v^{4}}{f^{2}}\right)
$$

Perturbation theory [117] was then used to determine the gauge boson masses up to $\mathcal{O}\left(\frac{v^{4}}{f^{2}}\right)$ and the mass eigenstates, normalized up to $\mathcal{O}\left(\frac{v^{2}}{f^{2}}\right)$.

Before EWSB (ignoring terms involving $v$ ), the neutral gauge boson mass matrix is diagonalized via:

$$
\begin{aligned}
& A_{0}^{3}=c_{g} A_{1}^{3}+s_{g} A_{2}^{3}, \\
& A_{H}^{3}=s_{g} A_{1}^{3}-c_{g} A_{2}^{3},
\end{aligned}
$$

where the mixing angle $s_{g}$ and $c_{g}$ are defined in Equation 4.51. Diagonalizing the neutral gauge boson mass matrix of Equation B.19 after EWSB leads to the following 
mass eigenstates up to $\mathcal{O}\left(\frac{v^{2}}{f^{2}+F^{2}}\right)$ :

$$
\begin{aligned}
\gamma & =s_{W} A_{0}^{3}+c_{W} B^{3} \\
Z & =c_{W} A_{0}^{3}-s_{W} B^{3}-x_{W} \frac{v^{2}}{f^{2}+F^{2}} A_{H}^{3} \\
Z^{\prime} & =A_{H}^{3}+x_{W} \frac{v^{2}}{f^{2}+F^{2}}\left(c_{W} A_{0}^{3}-s_{W} B^{3}\right)
\end{aligned}
$$

where the weak mixing angle is defined in Equation 4.52 .

Similarly, before EWSB (ignoring terms involving $v$ ), the charged gauge boson mass matrix is diagonalized via

$$
\begin{aligned}
& A_{0}^{j}=c_{g} A_{1}^{j}+s_{g} A_{2}^{j} \quad(j=1,2), \\
& A_{H}^{j}=s_{g} A_{1}^{j}-c_{g} A_{2}^{j} \quad(j=1,2),
\end{aligned}
$$

or in terms of the charge states:

$$
\begin{aligned}
A_{0}^{ \pm} & =\frac{1}{\sqrt{2}}\left(A_{0}^{1} \mp i A_{0}^{2}\right), \\
A_{H}^{ \pm} & =\frac{1}{\sqrt{2}}\left(A_{H}^{1} \mp i A_{H}^{2}\right) .
\end{aligned}
$$

Diagonalizing the charged gauge boson mass matrix of Equation B.20 after EWSB leads to the following mass eigenstates up to $\mathcal{O}\left(\frac{v^{2}}{f^{2}+F^{2}}\right)$ :

$$
\begin{aligned}
W^{ \pm} & =A_{0}^{ \pm}-c_{W} x_{W} \frac{v^{2}}{f^{2}+F^{2}} A_{H}^{ \pm} \\
W^{\prime \pm} & =A_{H}^{ \pm}+c_{W} x_{W} \frac{v^{2}}{f^{2}+F^{2}} A_{0}^{ \pm}
\end{aligned}
$$

The gauge boson masses are summarized in Equations 4.44-4.49. 


\section{B.4 Charge 2/3 Heavy Quark Mass Eigenstates}

The mass terms for the charge $2 / 3$ quarks may be written as

$$
L_{2 / 3 \text { mass }}=\left(u_{3}^{c}, U_{6}^{c}, U_{b 1}^{c}, U_{5}^{c}, U_{a 2}^{c}\right) M_{2 / 3}\left(\begin{array}{c}
q_{31} \\
Q_{6} \\
T_{b 2} \\
Q_{5} \\
T_{a 1}
\end{array}\right)
$$

where $M_{2 / 3}$ is a $5 \times 5$ mass matrix for the charge $2 / 3$ quarks. Fermion mass matrices are generally not symmetric nor hermitian, and must be diagonalized using a biunitary transformation [30]. That is, given $M_{2 / 3}$, there exist unitary matrices $S$ and $T$ such that

$$
S^{\dagger} M_{2 / 3} T=M_{2 / 3} \text { diag }
$$

Since $S$ and $T$ are unitary $\left(S S^{\dagger}=S^{\dagger} S=1\right.$ and $\left.T T^{\dagger}=T^{\dagger} T=1\right)$, it follows that

$$
\begin{aligned}
& S^{\dagger}\left(M_{2 / 3} M_{2 / 3}^{\dagger}\right) S=M_{2 / 3 \operatorname{diag}}^{2} \\
& T^{\dagger}\left(M_{2 / 3}^{\dagger} M_{2 / 3}\right) T=M_{2 / 3 \text { diag }}^{2}
\end{aligned}
$$


where

$$
M_{2 / 3 \text { diag }}^{2}=\left(\begin{array}{cccc}
M_{t}^{2} & & & \\
& & & \\
& M_{T_{6}}^{2} & & \\
& & & \\
& & M_{T_{b}^{(2 / 3)}}^{2} & \\
& & M_{T_{5}}^{2} & \\
& & & \\
& & & M_{T_{a}^{u}}^{2}
\end{array}\right)
$$

Furthermore, the mass eigenstates are given in terms of the gauge eigenstates as follows:

$$
\left(\begin{array}{c}
t \\
T_{6} \\
T_{b}^{(2 / 3)} \\
T_{5} \\
T_{a}^{u}
\end{array}\right)=A\left(\begin{array}{c}
q_{31} \\
Q_{6} \\
T_{b 2} \\
Q_{5} \\
T_{a 1}
\end{array}\right), \quad\left(\begin{array}{c}
t^{c} \\
T_{6}^{c} \\
\left(T_{b}^{(2 / 3)}\right)^{c} \\
T_{5}^{c} \\
\left(T_{a}^{u}\right)^{c}
\end{array}\right)=B\left(\begin{array}{c}
u_{3}^{c} \\
U_{6}^{c} \\
U_{b 1}^{c} \\
U_{5}^{c} \\
U_{a 2}^{c}
\end{array}\right)
$$

where $A \equiv S^{\dagger}$ and $B \equiv T^{\dagger}$.

Diagonalizing $M_{2 / 3}^{\dagger} M_{2 / 3}$ and $M_{2 / 3} M_{2 / 3}^{\dagger}$ using perturbation theory, following the same procedure that was used to diagonalize the gauge boson mass matrices, yields the masses of the charge 2/3 quarks up to $\mathcal{O}\left(\frac{v^{4}}{f^{2}}\right)$, summarized in Equations 4.79-4.83, and the mass eigenstates up to $\mathcal{O}\left(\frac{v^{2}}{f^{2}}\right)$.

The left-handed mass eigenstates for the charge $2 / 3$ heavy quarks, normalized up to $\mathcal{O}\left(\frac{v^{2}}{f^{2}}\right)$, were determined to be: 
$t=A_{11} q_{31}+A_{12} Q_{6}+A_{13} T_{b 2}+A_{14} Q_{5}+A_{15} T_{a 1}$

where

$$
\begin{aligned}
& A_{11}=1-\frac{y_{2}^{2}\left(2 y_{1}^{2}-y_{3}^{2}\right)^{2}}{2\left(y_{1}^{2}+y_{2}^{2}\right)\left(y_{1}^{2}+y_{3}^{2}\right)^{2}} \frac{v_{1}^{2}}{f^{2}}-\frac{2 y_{2}^{2}}{y_{1}^{2}+y_{2}^{2}} \frac{v_{2}^{2}}{f^{2}} \\
& A_{12}=\frac{2 y_{2}}{\sqrt{y_{1}^{2}+y_{2}^{2}}} \frac{v_{2}}{f} \\
& A_{13}=-\frac{y_{2}\left(2 y_{1}^{2}+5 y_{3}^{2}\right)}{\left(y_{1}^{2}+y_{3}^{2}\right) \sqrt{y_{1}^{2}+y_{2}^{2}}} \frac{v_{1}^{2}}{f^{2}}-\frac{2 y_{2}}{\sqrt{y_{1}^{2}+y_{2}^{2}}} \frac{v_{2}^{2}}{f^{2}} \\
& A_{14}=\frac{y_{2}\left(2 y_{1}^{2}-y_{3}^{2}\right)}{\left(y_{1}^{2}+y_{3}^{2}\right) \sqrt{y_{1}^{2}+y_{2}^{2}}} \frac{v_{1}}{f} \\
& A_{15}=-\frac{y_{1} y_{2}\left[y_{3}^{2}\left(5 y_{1}^{2}-4 y_{2}^{2}\right)+2 y_{1}^{2}\left(y_{1}^{2}+y_{2}^{2}\right)\right]}{\left(y_{1}^{2}+y_{2}^{2}\right)^{2}\left(y_{1}^{2}+y_{3}^{2}\right)} \frac{v_{1}^{2}}{f^{2}}-\frac{2 y_{1} y_{2}}{y_{1}^{2}+y_{2}^{2}} \frac{v_{2}^{2}}{f^{2}}
\end{aligned}
$$

$T_{6}=A_{21} q_{31}+A_{22} Q_{6}+A_{23} T_{b 2}+A_{24} Q_{5}+A_{25} T_{a 1}$

where

$$
\begin{aligned}
& A_{21}=-\frac{2 y_{2}}{\sqrt{y_{1}^{2}+y_{2}^{2}}} \frac{v_{2}}{f} \\
& A_{22}=1-2 \frac{v_{2}^{2}}{f^{2}} \\
& A_{23}=0 \\
& A_{24}=3 \frac{v_{1} v_{2}}{f^{2}} \\
& A_{25}=-\frac{2 y_{1}}{\sqrt{y_{1}^{2}+y_{2}^{2}}} \frac{v_{2}}{f}
\end{aligned}
$$



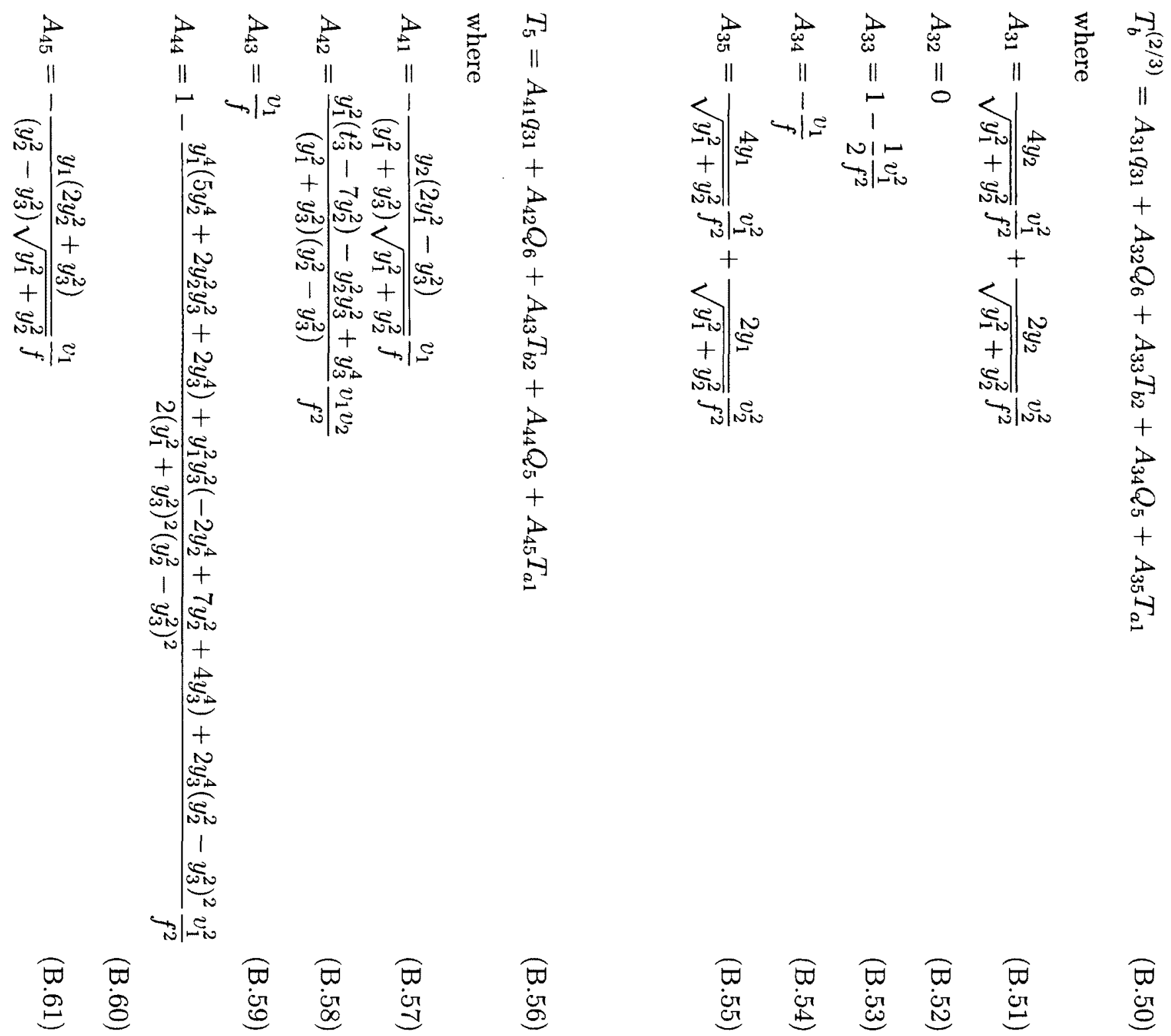

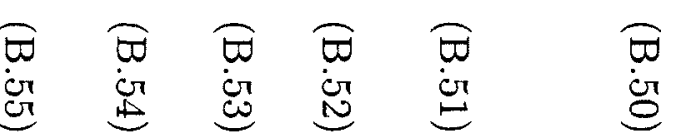




$$
T_{a}^{u}=A_{51} q_{31}+A_{52} Q_{6}+A_{53} T_{b 2}+A_{54} Q_{5}+A_{55} T_{a 1}
$$

where

$$
\begin{aligned}
& A_{51}=-\frac{y_{1} y_{2}\left[2 y_{1}^{2}\left(y_{2}^{2}+2 y_{3}^{2}\right)+2 y_{2}^{4}-5 y_{2}^{2} y_{3}^{2}\right]}{\left(y_{1}^{2}+y_{2}^{2}\right)^{2}\left(y_{2}^{2}-y_{3}^{2}\right)} \frac{v_{1}^{2}}{f^{2}}-\frac{2 y_{1} y_{2}}{y_{1}^{2}+y_{2}^{2}} \frac{v_{2}^{2}}{f^{2}} \\
& A_{52}=\frac{2 y_{1}}{\sqrt{y_{1}^{2}+y_{2}^{2}}} \frac{v_{2}}{f} \\
& A_{53}=\frac{y_{1}\left(5 y_{3}^{2}-2 y_{2}^{2}\right)}{\left(y_{2}^{2}-y_{3}^{2}\right) \sqrt{y_{1}^{2}+y_{2}^{2}}} \frac{v_{1}^{2}}{f^{2}}-\frac{2 y_{1}}{\sqrt{y_{1}^{2}+y_{2}^{2}}} \frac{v_{2}^{2}}{f^{2}} \\
& A_{54}=\frac{y_{1}\left(2 y_{2}^{2}+y_{3}^{2}\right)}{\left(y_{2}^{2}-y_{3}^{2}\right) \sqrt{y_{1}^{2}+y_{2}^{2}}} \frac{v_{1}}{f} \\
& A_{55}=1-\frac{y_{1}^{2}\left(2 y_{2}^{2}+y_{3}^{2}\right)^{2}}{2\left(y_{1}^{2}+y_{2}^{2}\right)\left(y_{2}^{2}-y_{3}^{2}\right)^{2}} \frac{v_{1}^{2}}{f^{2}}-\frac{2 y_{1}^{2}}{y_{1}^{2}+y_{2}^{2}} \frac{v_{2}^{2}}{f^{2}}
\end{aligned}
$$

The right-handed mass eigenstates for the charge $2 / 3$ heavy quarks, normalized up to $\mathcal{O}\left(\frac{v^{2}}{f^{2}}\right)$, were determined to be:

$$
t^{c}=B_{11} u_{3}^{c}+B_{12} U_{6}^{c}+B_{13} U_{b 1}^{c}+B_{14} U_{5}^{c}+B_{15} U_{a 2}^{c}
$$

where

$$
\begin{aligned}
& B_{11}=1-\frac{y_{3}^{2}\left(8 y_{1}^{4}+4 y_{1}^{2} y_{2}^{2}+5 y_{2}^{4}\right)}{2\left(y_{1}^{2}+y_{2}^{2}\right)^{2}\left(y_{1}^{2}+y_{3}^{2}\right)} \frac{v_{1}^{2}}{f^{2}} \\
& B_{12}=-\frac{y_{3}\left(4 y_{1}^{2}+7 y_{2}^{2}\right)}{\left(y_{1}^{2}+y_{2}^{2}\right) \sqrt{y_{1}^{2}+y_{3}^{2}} \frac{v_{1}}{f}} \\
& B_{13}=\frac{2 y_{3}}{\sqrt{y_{1}^{2}+y_{3}^{2}}} \frac{v_{1}}{f} \\
& B_{14}=-\frac{y_{1} y_{2}\left[4 y_{1}^{4}+y_{1}^{2}\left(7 y_{2}^{2}+4 y_{3}^{2}\right)-2 y_{2}^{2} y_{3}^{2}\right]}{\left(y_{1}^{2}+y_{2}^{2}\right)\left(y_{1}^{2}+y_{3}^{2}\right)^{2}} \frac{v_{1}^{2}}{f^{2}} \\
& B_{15}=\frac{y_{3}\left(2 y_{1}^{2}-y_{2}^{2}\right)}{\left(y_{1}^{2}+y_{2}^{2}\right) \sqrt{y_{1}^{2}+y_{3}^{2}}} \frac{v_{1}}{f}
\end{aligned}
$$




$$
T_{6}^{c}=B_{21} u_{3}^{c}+B_{22} U_{6}^{c}+B_{23} U_{b 1}^{c}+B_{24} U_{5}^{c}+B_{25} U_{a 2}^{c}
$$

where

$$
\begin{aligned}
& B_{21}=\frac{6 y_{3}}{\sqrt{y_{1}^{2}+y_{3}^{2}}} \frac{v_{1} v_{2}}{f^{2}} \\
& B_{22}=1-\frac{1}{2} \frac{v_{2}^{2}}{f^{2}} \\
& B_{23}=0 \\
& B_{24}=\frac{6 y_{1}}{\sqrt{y_{1}^{2}+y_{3}^{2}}} \frac{v_{1} v_{2}}{f^{2}} \\
& B_{25}=-\frac{v_{2}}{f}
\end{aligned}
$$

$$
\left(T_{b}^{(2 / 3)}\right)^{c}=B_{31} u_{3}^{c}+B_{32} U_{6}^{c}+B_{33} U_{b 1}^{c}+B_{34} U_{5}^{c}+B_{35} U_{a 2}^{c}
$$

where

$$
\begin{aligned}
& B_{31}=-\frac{2 y_{3}}{\sqrt{y_{1}^{2}+y_{3}^{2}}} \frac{v_{1}}{f} \\
& B_{32}=0 \\
& B_{33}=1-2 \frac{v_{1}^{2}}{f^{2}} \\
& B_{34}=-\frac{2 y_{1}}{\sqrt{y_{1}^{2}+y_{3}^{2}}} \frac{v_{1}}{f} \\
& B_{35}=\frac{5}{2} \frac{v_{1}^{2}}{f^{2}}+\frac{1}{2} \frac{v_{2}^{2}}{f^{2}}
\end{aligned}
$$




$$
T_{5}^{c}=B_{41} u_{3}^{c}+B_{42} U_{6}^{c}+B_{43} U_{b 1}^{c}+B_{44} U_{5}^{c}+B_{45} U_{a 2}^{c}
$$

where

$$
\begin{aligned}
& B_{41}=\frac{y_{1} y_{3}\left[2 y_{1}^{2}\left(y_{2}^{2}+2 y_{3}^{2}\right)-7 y_{2}^{2} y_{3}^{2}+4 y_{3}^{4}\right] \frac{v_{1}^{2}}{\left.f^{2}+y_{3}^{2}\right)^{2}\left(y_{2}^{2}-y_{3}^{2}\right)}}{f_{42}}=-\frac{y_{1}\left(7 y_{2}^{2}-4 y_{3}^{2}\right)}{\left(y_{2}^{2}-y_{3}^{2}\right) \sqrt{y_{1}^{2}+y_{3}^{2}}} \frac{v_{1} v_{2}}{f^{2}} \\
& B_{43}=\frac{2 y_{1}}{\sqrt{y_{1}^{2}+y_{3}^{2}}} \frac{v_{1}}{f} \\
& B_{44}=1-\frac{y_{1}^{2}\left(5 y_{2}^{4}-4 y_{2}^{2} y_{3}^{2}+8 y_{3}^{4}\right)}{2\left(y_{1}^{2}+y_{3}^{2}\right)\left(y_{2}^{2}-y_{3}^{2}\right)^{2}} \frac{v_{1}^{2}}{f^{2}} \\
& B_{45}=-\frac{y_{1}\left(y_{2}^{2}+2 y_{3}^{2}\right)}{\left(y_{2}^{2}-y_{3}^{2}\right) \sqrt{y_{1}^{2}+y_{3}^{2}}} \frac{v_{1}}{f}
\end{aligned}
$$

$$
\left(T_{a}^{u}\right)^{c}=B_{51} u_{3}^{c}+B_{52} U_{6}^{c}+B_{53} U_{b 1}^{c}+B_{54} U_{5}^{c}+B_{55} U_{a 2}^{c}
$$

where

$$
\begin{aligned}
& B_{51}=-\frac{y_{3}\left(2 y_{1}^{2}-y_{2}^{2}\right)}{\left(y_{1}^{2}+y_{2}^{2}\right) \sqrt{y_{1}^{2}+y_{3}^{2}}} \frac{v_{1}}{f} \\
& B_{52}=\frac{v_{2}}{f} \\
& B_{53}=-\frac{1}{2}\left[1-\frac{12 y_{1}^{2} y_{3}^{2}}{\left(y_{1}^{2}+y_{2}^{2}\right)\left(y_{2}^{2}-y_{3}^{2}\right)}\right] \frac{v_{1}^{2}}{f^{2}}-\frac{1}{2} \frac{v_{2}^{2}}{f^{2}} \\
& B_{54}=\frac{y_{1}\left(y_{2}^{2}+2 y_{3}^{2}\right)}{\left(y_{2}^{2}-y_{3}^{2}\right) \sqrt{y_{1}^{2}+y_{3}^{2}} \frac{v_{1}}{f}} \\
& B_{55}=1-\frac{1}{2}\left[1+\frac{3 y_{1}^{2} y_{3}^{2}\left[y_{1}^{2}\left(2 y_{2}^{2}+y_{3}^{2}\right)+5 y_{2}^{4}-2 y_{2}^{2} y_{3}^{2}\right]}{\left(y_{1}^{2}+y_{2}^{2}\right)^{2}\left(y_{2}^{2}-y_{3}^{2}\right)^{2}}\right] \frac{v_{1}^{2}}{f^{2}}-\frac{1}{2} \frac{v_{2}^{2}}{f^{2}}
\end{aligned}
$$

The physical mass eigenstates of the charge $2 / 3$ quarks are defined in terms of the gauge eigenstates in Equations B.38-B.97. The gauge eigenstates can easily be expressed in terms of the mass eigenstates by inverting the relations of Equation B.37. 
Using the fact that the $A$ and $B$ matrices are unitary and, in this particular case, real gives $A A^{\dagger}=1 \Longrightarrow A^{-1}=A^{\dagger}=A^{T}$, and similarly for the matrix $B$. Therefore:

$$
\left(\begin{array}{c}
q_{31} \\
Q_{6} \\
T_{b 2} \\
Q_{5} \\
T_{a 1}
\end{array}\right)=A^{T}\left(\begin{array}{c}
t \\
T_{6} \\
T_{b}^{(2 / 3)} \\
T_{5} \\
T_{a}^{u}
\end{array}\right),\left(\begin{array}{c}
u_{3}^{c} \\
U_{6}^{c} \\
U_{b 1}^{c} \\
U_{5}^{c} \\
U_{a 2}^{c}
\end{array}\right)=B^{T}\left(\begin{array}{c}
t^{c} \\
T_{6}^{c} \\
\left(T_{b}^{(2 / 3)}\right)^{c} \\
T_{5}^{c} \\
\left(T_{a}^{u}\right)^{c}
\end{array}\right)
$$

This can be written more explicitly as

$$
\begin{aligned}
q_{31} & =A_{11} t+A_{21} T_{6}+A_{31} T_{b}^{(2 / 3)}+A_{41} T_{5}+A_{51} T_{a}^{u} \\
Q_{6} & =A_{12} t+A_{22} T_{6}+A_{32} T_{b}^{(2 / 3)}+A_{42} T_{5}+A_{52} T_{a}^{u} \\
T_{b 2} & =A_{13} t+A_{23} T_{6}+A_{33} T_{b}^{(2 / 3)}+A_{43} T_{5}+A_{53} T_{a}^{u} \\
Q_{5} & =A_{14} t+A_{24} T_{6}+A_{34} T_{b}^{(2 / 3)}+A_{44} T_{5}+A_{54} T_{a}^{u} \\
T_{a 1} & =A_{15} t+A_{25} T_{6}+A_{35} T_{b}^{(2 / 3)}+A_{45} T_{5}+A_{55} T_{a}^{u}
\end{aligned}
$$

and

$$
\begin{aligned}
u_{3}^{c} & =B_{11} t^{c}+B_{21} T_{6}^{c}+B_{31}\left(T_{b}^{(2 / 3)}\right)^{c}+B_{41} T_{5}^{c}+B_{51}\left(T_{a}^{u}\right)^{c} \\
U_{6}^{c} & =B_{12} t^{c}+B_{22} T_{6}^{c}+B_{32}\left(T_{b}^{(2 / 3)}\right)^{c}+B_{42} T_{5}^{c}+B_{52}\left(T_{a}^{u}\right)^{c} \\
U_{b 1}^{c} & =B_{13} t^{c}+B_{23} T_{6}^{c}+B_{33}\left(T_{b}^{(2 / 3)}\right)^{c}+B_{43} T_{5}^{c}+B_{53}\left(T_{a}^{u}\right)^{c} \\
U_{5}^{c} & =B_{14} t^{c}+B_{24} T_{6}^{c}+B_{34}\left(T_{b}^{(2 / 3)}\right)^{c}+B_{44} T_{5}^{c}+B_{54}\left(T_{a}^{u}\right)^{c} \\
U_{a 2}^{c} & =B_{15} t^{c}+B_{25} T_{6}^{c}+B_{35}\left(T_{b}^{(2 / 3)}\right)^{c}+B_{45} T_{5}^{c}+B_{55}\left(T_{a}^{u}\right)^{c}
\end{aligned}
$$


where the $A$ and $B$ coefficients are listed above.

Writing the gauge eigenstates in terms of the mass eigenstates in this manner is useful for determining the interactions between fermions and the gauge bosons and scalars of the model, as discussed in Section B.5.

\section{B.5 Calculation of Feynman Rules}

As the Bestest Little Higgs model contains a very large number of interaction vertices, the complete set of Feynman rules is not listed in this thesis. Instead, the procedure that was used to derive them is explained in this section. Calculating the Feynman rules of the Bestest Little Higgs model was accomplished by writing the gauge eigenstates in terms of the mass eigenstates for the fermions, gauge bosons and scalars. These relations were then substituted into the appropriate Lagrangian and terms involving the physical particles were then written out. The coefficients of these terms give the Feynman rules needed to calculate the interactions between the given particles. These Feynman rules were then implemented in MadGraph5 version 1.1.0 [96].

Inverting the relations of Equations B.6, B.7 and B.10 gives the Higgs doublet 
gauge eigenstates written in terms of the mass eigenstates as follows:

$$
\begin{aligned}
& h_{11}=\cos \alpha h^{0}-\sin \alpha H^{0} \\
& h_{12}=-\cos \beta A^{0}+\sin \beta G^{0} \\
& h_{13}=-\frac{1}{\sqrt{2}}\left[\cos \beta\left(H^{+}+H^{-}\right)-\sin \beta\left(G^{+}+G^{-}\right)\right] \\
& h_{14}=\frac{i}{\sqrt{2}}\left[\cos \beta\left(H^{+}-H^{-}\right)-\sin \beta\left(G^{+}-G^{-}\right)\right] \\
& h_{21}=\sin \alpha h^{0}+\cos \alpha H^{0} \\
& h_{22}=\sin \beta A^{0}+\cos \beta G^{0} \\
& h_{23}=\frac{1}{\sqrt{2}}\left[\sin \beta\left(H^{+}+H^{-}\right)+\cos \beta\left(G^{+}+G^{-}\right)\right] \\
& h_{24}=-\frac{i}{\sqrt{2}}\left[\sin \beta\left(H^{+}-H^{-}\right)+\cos \beta\left(G^{+}-G^{-}\right)\right]
\end{aligned}
$$

Inverting the relations of Equations B.14-B.17 gives the scalar triplet gauge eigenstates written in terms of the mass eigenstates as follows:

$$
\begin{aligned}
\phi_{p h y s}^{1} & =\frac{1}{\sqrt{2}}\left(\phi^{+}+\phi^{-}\right) \\
\phi_{p h y s}^{2} & =\frac{i}{\sqrt{2}}\left(\phi^{+}-\phi^{-}\right) \\
\phi_{p h y s}^{3} & =\phi^{0} \\
\eta_{p h y s}^{1} & =\frac{1}{\sqrt{2}}\left(\eta^{+}+\eta^{-}\right) \\
\eta_{p h y s}^{2} & =\frac{i}{\sqrt{2}}\left(\eta^{+}-\eta^{-}\right) \\
\eta_{p h y s}^{3} & =\phi^{0}
\end{aligned}
$$

Inverting the relations of Equations B.23-B.25 and B.30-B.31 gives the gauge 
boson gauge eigenstates written in terms of the mass eigenstates as follows:

$$
\begin{aligned}
& A_{1}^{1}=\frac{1}{\sqrt{2}}\left[\left(c_{g}-s_{g} c_{W} x_{W} \frac{v^{2}}{f^{2}+F^{2}}\right)\left(W^{+}+W^{-}\right)\right. \\
& \left.+\left(s_{g}-c_{g} c_{W} x_{W} \frac{v^{2}}{f^{2}+F^{2}}\right)\left(W^{\prime+}+W^{\prime-}\right)\right] \\
& A_{2}^{1}=\frac{1}{\sqrt{2}}\left[\left(s_{g}+c_{g} c_{W} x_{W} \frac{v^{2}}{f^{2}+F^{2}}\right)\left(W^{+}+W^{-}\right)\right. \\
& \left.-\left(c_{g}-s_{g} c_{W} x_{W} \frac{v^{2}}{f^{2}+F^{2}}\right)\left(W^{\prime+}+W^{\prime-}\right)\right] \\
& A_{1}^{2}=\frac{i}{\sqrt{2}}\left[\left(c_{g}-s_{g} c_{W} x_{W} \frac{v^{2}}{f^{2}+F^{2}}\right)\left(W^{+}-W^{-}\right)\right. \\
& \left.+\left(s_{g}+c_{g} c_{W} x_{W} \frac{v^{2}}{f^{2}+F^{2}}\right)\left(W^{\prime+}-W^{\prime-}\right)\right] \\
& A_{2}^{2}=\frac{i}{\sqrt{2}}\left[\left(s_{g}+c_{g} c_{W} x_{W} \frac{v^{2}}{f^{2}+F^{2}}\right)\left(W^{+}-W^{-}\right)\right. \\
& \left.-\left(c_{g}-s_{g} c_{W} x_{W} \frac{v^{2}}{f^{2}+F^{2}}\right)\left(W^{\prime+}-W^{\prime-}\right)\right] \\
& A_{1}^{3}=c_{g} s_{W} \gamma+\left(c_{g} c_{W}-s_{g} x_{W} \frac{v^{2}}{f^{2}+F^{2}}\right) Z+\left(s_{g}+c_{g} c_{W} x_{W} \frac{v^{2}}{f^{2}+F^{2}}\right) Z^{\prime} \\
& A_{2}^{3}=s_{g} s_{W} \gamma+\left(s_{g} c_{W}+c_{g} x_{W} \frac{v^{2}}{f^{2}+F^{2}}\right) Z+\left(-c_{g}+s_{g} c_{W} x_{W} \frac{v^{2}}{f^{2}+F^{2}}\right) Z^{\prime} \\
& B^{3}=c_{W} \gamma-s_{W} Z-s_{W} x_{W} \frac{v^{2}}{f^{2}+F^{2}} Z^{\prime}
\end{aligned}
$$

The most important Feynman rules for studying heavy quark production are the 3-point fermion-fermion-scalar (FFS) and fermion-fermion-gauge (FFV) interactions. The FFS interactions were derived from the Yukawa Lagrangian of Equation 4.58, whereas the FFV interactions were derived from

$$
\mathcal{L}_{F F V}=i Q^{\dagger} \bar{\tau}^{\mu} D_{\mu} Q+i Q_{a}^{\prime \dagger} \bar{\tau}^{\mu} D_{\mu} Q_{a}^{\prime}-i U^{c \dagger} \tau^{\mu} D_{\mu} U^{c}-i U_{5}^{\prime c \dagger} \tau^{\mu} D_{\mu} U_{5}^{\prime c}-i U_{b}^{c \dagger} \tau^{\mu} D_{\mu} U_{b}^{c}
$$

where $\tau^{\mu}$ and $\bar{\tau}^{\mu}$ are defined in Equations A.5-A.6, and the covariant derivatives of 
the fermion fields are given by

$$
\begin{aligned}
D_{\mu} Q & =\partial_{\mu} Q+i g_{A} A_{1 \mu}^{a} T^{a} Q+i g^{\prime} B_{\mu}^{3}\left(T^{3}+\frac{2}{3} \mathbb{1}_{6}\right) Q \\
D_{\mu} Q_{a}^{\prime} & =\partial_{\mu} Q_{a}^{\prime}+i g_{A} A_{1 \mu}^{a} T^{a} Q_{a}^{\prime}+i g^{\prime} B_{\mu}^{3}\left(\frac{1}{6}\right) Q_{a}^{\prime} \\
D_{\mu} U^{c} & =\partial_{\mu} U^{c}+i g_{B} A_{2 \mu}^{a} T^{a} U^{c}+i g^{\prime} B_{\mu}^{3}\left(T^{3}-\frac{2}{3} \mathbb{1}_{6}\right) U^{c} \\
D_{\mu} U_{5}^{\prime c} & =\partial_{\mu} U_{5}^{\prime c}+i g^{\prime} B_{\mu}^{3}\left(-\frac{2}{3}\right) U_{5}^{\prime c} \\
D_{\mu} U_{b}^{c} & =\partial_{\mu} U_{b}^{c}+i g^{\prime} B_{\mu}^{3}\left(\frac{1}{3}\right) U_{b}^{c} .
\end{aligned}
$$

The two-component Weyl fermion notation of Equation B.130 is converted to fourcomponent Dirac fermion notation via the following relations [95]:

$$
\psi_{i, L}^{\dagger} \bar{\tau}_{\mu} \psi_{j, L}=\bar{\Psi}_{i} \gamma_{\mu} P_{L} \Psi_{j}, \quad \psi_{i, R}^{\dagger} \tau_{\mu} \psi_{j, R}=\bar{\Psi}_{i} \gamma_{\mu} P_{R} \Psi_{j}
$$

where

$$
\Psi \equiv\left(\begin{array}{c}
\psi_{L} \\
\psi_{R}
\end{array}\right)
$$

is a four-component Dirac spinor. $\psi_{L}$ corresponds to the components of the $Q$ and $Q_{a}^{\prime}$ multiplets, defined in Equations 4.53 and 4.59, respectively, while $\psi_{R}$ corresponds to the components of the $U^{c}, U_{5}^{\prime c}$ and $U_{b}^{c}$ multiplets, defined in Equations $4.55,4.60$ and 4.62 , respectively. 


\section{References}

[1] K. Nakamura et al. (Particle Data Group), "Review of Particle Physics," J. Phys. G, vol. 37, p. 075021, 2010.

[2] P. W. Higgs, "Broken symmetries and the masses of gauge bosons," Phys. Rev. Lett., vol. 13, pp. 508-509, 1964.

[3] The ATLAS Collaboration, "Observation of an Excess of Events in the Search for the Standard Model Higgs boson with the ATLAS detector at the LHC," ATLAS-CONF-2012-093, 2012.

[4] The CMS Collaboration, "Observation of a resonance with a mass near 125 $\mathrm{GeV}$ in the search for the Higgs boson in $p p$ collisions at $\sqrt{s}=7 \mathrm{TeV}$ and 8 TeV," CMS-HIG-12-020, 2012.

[5] S. Chatrchyan et al. [CMS Collaboration], "Search for Supersymmetry at the LHC in Events with Jets and Missing Transverse Energy," 2011, [arXiv:1109.2352 [hep-ex]].

[6] G. Aad et al. [ATLAS Collaboration], "Search for new phenomena in final states with large jet multiplicities and missing transverse momentum using $\sqrt{s}=7$ $\mathrm{TeV} p p$ collisions with the ATLAS detector," JHEP, vol. 1111, p. 099, 2011, [arXiv:1110.2299 [hep-ex]].

[7] J. A. Conley, J. S. Gainer, J. L. Hewett, M. P. Le, and T. G. Rizzo, "Supersymmetry Without Prejudice at the $7 \mathrm{TeV}$ LHC," Submitted to Physical Review D, 2011, [arXiv:1103.1697 [hep-ph]].

[8] M. Papucci, J. T. Ruderman, and A. Weiler, "Natural SUSY Endures," 2011, [arXiv:1110.6926 [hep-ph]].

[9] S. B. Kim et al. [CDF Collaboration], "Observation of the Top Quark," FNAL/C-95/276-E, 1995. 
[10] D. E. Morrissey, T. Plehn, and T. M. P. Tait, "New Physics at the LHC," 2009, [arXiv:0912.3259 [hep-ph]].

[11] E. Accomando et al., "Workshop on CP Studies and Non-Standard Higgs Physics," 2006, [arXiv:hep-ph/0608079].

[12] P. Nath et al., "The Hunt for New Physics at the Large Hadron Collider," 2010, [arXiv:1001.2693 [hep-ph]].

[13] N. Arkani-Hamed, A. G. Cohen, and H. Georgi, "Electroweak symmetry breaking from dimensional deconstruction," Phys. Lett. B, vol. 513, p. 232, 2001, [arXiv:hep-ph/0105239].

[14] H.-C. Cheng, C. T. Hill, S. Pokorski, and J. Wang, "Standard model in the latticized bulk," Phys. Rev. D, vol. 64, p. 065007, 2001.

[15] H.-C. Cheng, C. T. Hill, and J. Wang, "Dynamical electroweak breaking and latticized extra dimensions," Phys. Rev. D, vol. 64, p. 095003, 2001.

[16] C. T. Hill, S. Pokorski, and J. Wang, "Gauge invariant effective lagrangian for kaluza-klein modes," Phys. Rev. D, vol. 64, p. 105005, 2001.

[17] N. Arkani-Hamed, A. G. Cohen, E. Katz, and A. E. Nelson, "The littlest Higgs," $J H E P$, vol. 0207, p. 034, 2002, [arXiv:hep-ph/0206021].

[18] H. Georgi and M. Machacek, "Doubly Charged Higgs Bosons," Nucl. Phys. B, vol. 262, p. 463, 1985.

[19] R. N. Mohapatra and J. C. Pati, "Left-Right Gauge Symmetry and an Isoconjugate Model of CP Violation," Phys. Rev. D, vol. 11, p. 566, 1975.

[20] G. Senjanovic and R. N. Mohapatra, "Exact Left-Right Symmetry And Spontaneous Violation Of Parity," Phys. Rev. D, vol. 12, p. 1502, 1975.

[21] M. Schmaltz, D. Stolarski, and J. Thaler, "The Bestest Little Higgs," JHEP, vol. 1009, p. 018, 2010, [arXiv:1006.1356 [hep-ph]].

[22] S. Glashow, "Partial Symmetries of Weak Interactions," Nucl. Phys., vol. 22, pp. $579-588,1961$.

[23] S. Weinberg, "A Model of Leptons," Phys. Rev. Lett., vol. 19, pp. $1264-1266$, 1967. 
[24] A. Salam, Elementary Particle Theory. Almqvist and Wiksells, Stockholm, 1969.

[25] H. Fritzsch, M. Gell-Mann, and H. Leutwyler, "Advantages of the Color Octet Gluon Picture," Phys. Lett. B, vol. 47, pp. 365-368, 1973.

[26] D. J. Gross and F. Wilczek, "Asymptotically Free Gauge Theories. I," Phys. Rev. D, vol. 8, pp. $3633-3652,1973$.

[27] P. Langacker, "Introduction to the Standard Model and Electroweak Physics," 2009, [arXiv:0901.0241v1 [hep-ph]].

[28] S. Dawson, "Introduction to Electroweak Symmetry Breaking," 1999, [arXiv.org/hep-ph/9901280].

[29] M. E. Peskin and D. V. Schroeder, An Introduction to Quantum Field Theory. U.S.A.: Westview Press, 1995.

[30] T.-P. Cheng and L.-F. Li, Gauge Theory of Elementary Particle Physics. Oxford: Oxford University Press, 1984.

[31] J. Goldstone, A. Salam, and S. Weinberg, "Broken Symmetries," Phys. Rev., vol. 127, pp. 965-970, 1962.

[32] E. Fermi, Atti. Soc. It. Prog. Scient., vol. 2, pp. 7 - 14, 1933.

[33] R. Barate et al. [LEP Working Group for Higgs boson searches and ALEPH Collaboration and L3 Collaboration and OPAL Collaboration], "Search for the standard model Higgs boson at LEP," Phys. Lett. B, vol. 565, p. 61, 2003, [arXiv:hep-ex/0306033].

[34] G. Aad et al. [ATLAS Collaboration], "Combined search for the Standard Model Higgs boson using up to $4.9 \mathrm{fb}^{-1}$ of $p p$ collision data at $\sqrt{s}=7 \mathrm{TeV}$ with the ATLAS detector at the LHC," Phys. Lett. B, vol. 710, pp. 49-66, 2012, [arXiv:1202.1408v3 [hep-ex]].

[35] S. Chatrchyan et al. [CMS Collaboration], "Combined results of searches for the standard model Higgs boson in $p p$ collisions at $\sqrt{s}=7 \mathrm{TeV}$," Phys. Lett. $B$, vol. 710, pp. 26-48, 2012, [arXiv:1202.1488v1 [hep-ex]].

[36] S. Dawson, "Electroweak Symmetry Breaking circa 2005," Int. J. Mod. Phys. $A$, vol. 21, pp. $1629-1641,2006$. 
[37] G. Aad et al. [ATLAS Collaboration], "Search for Supersymmetry Using Final States with One Lepton, Jets, and Missing Transverse Momentum with the ATLAS Detector in $\sqrt{s}=7 \mathrm{TeV} p p$ Collisions," Phys. Rev. Lett., vol. 106, p. 131802, 2011, [arXiv:1102.2357v2 [hep-ex]].

[38] M. Schmaltz and D. Tucker-Smith, "Little Higgs Review," Ann. Rev. Nucl. Part. Sci., vol. 55, p. 229, 2005, [arXiv:hep-ph/0502182].

[39] T. Han, H. E. Logan, B. McElrath, and L. T. Wang, "Phenomenology of the little Higgs model," Phys. Rev. D, vol. 67, p. 095004, 2003, [arXiv:hep$\mathrm{ph} / 0301040]$.

[40] T. Han, H. E. Logan, and L. T. Wang, "Smoking-gun signatures of little Higgs models," JHEP, vol. 0601, p. 099, 2006, [arXiv:hep-ph/0506313].

[41] M. Perelstein, "Little Higgs models and their phenomenology," Prog. Part. Nucl. Phys., vol. 58, p. 247, 2007, [arXiv:hep-ph/0512128].

[42] M. Schmaltz, "The Simplest Little Higgs," JHEP, vol. 0408, p. 056, 2004, [arXiv:hep-ph/0407143].

[43] H.-C. Cheng and I. Low, "TeV Symmetry and the Little Hierarchy Problem," $J H E P$, vol. 0309, p. 051, 2003, [arXiv:hep-ph/0308199].

[44] H.-C. Cheng and I. Low, "Little hierarchy, little Higgses, and a little symmetry," JHEP, vol. 0408, p. 061, 2004, [arXiv:hep-ph/0405243].

[45] I. Low, "T-parity and the littlest Higgs," JHEP, vol. 0410, p. 067, 2004, [arXiv:hep-ph/0409025].

[46] R. Barbieri and A. Strumia, "The 'LEP paradox'," 2000, [arXiv:hep$\mathrm{ph} / 0007265]$.

[47] M. Schmaltz and J. Thaler, "Collective Quartics and Dangerous Singlets in Little Higgs," JHEP, vol. 0903, p. 137, 2009, [arXiv:0812.2477 [hep-ph]].

[48] G. Burdman, M. Perelstein, and A. Pierce, "Collider tests of the little Higgs model," Phys. Rev. Lett., vol. 90, p. 241802, 2003, [Erratum-ibid. vol. 92, 049903 (2004)], [arXiv:hep-ph/0212228].

[49] G. Azuelos et al., "Exploring little Higgs models with ATLAS at the LHC," Eur. Phys. J. C, vol. 39, p. 13, 2005, [arXiv:hep-ph/0402037]. 
[50] J. Hubisz and P. Meade, "Phenomenology of the littlest Higgs with $T$-parity," Phys. Rev. D, vol. 71, p. 035016, 2005, [arXiv:hep-ph/0411264].

[51] S. R. Coleman and E. J. Weinberg, "Radiative Corrections as the Origin of Spontaneous Symmetry Breaking," Phys. Rev. D, vol. 7, pp. 1888 - 1910, 1973.

[52] E. Asakawa, S. Kanemura, and J. Kanzaki, "Potential for measuring the $H^{ \pm} W^{\mp} Z^{0}$ vertex from $W Z$ fusion at the Large Hadron Collider," Phys. Rev. $D$, vol. 75, p. 075022, 2007, [arXiv:hep-ph/0612271].

[53] F. Pisano and V. Pleitez, "An $S U(3) \times U(1)$ model for electroweak interactions," Phys. Rev. D, vol. 46, p. 410, 1992, [arXiv:hep-ph/9206242].

[54] P. H. Frampton, "Chiral dilepton model and the flavor question," Phys. Rev. Lett., vol. 69, p. 2889, 1992.

[55] J. E. Cieza Montalvo, N. V. Cortez, J. Sa Borges and M. D. Tonasse, "Searching for doubly charged Higgs bosons at the LHC in a 3-3-1 model," Nucl. Phys. B, vol. 756, pp. 1-15, 2006, [arXiv:hep-ph/0606243].

[56] C. Csaki, C. Grojean, H. Murayama, L. Pilo, and J. Terning, "Gauge theories on an interval: Unitarity without a Higgs," Phys. Rev. D, vol. 69, p. 055006, 2004, [arXiv:hep-ph/0305237].

[57] C. Csaki, C. Grojean, L. Pilo, and J. Terning, "Towards a realistic model of Higgsless electroweak symmetry breaking," Phys. Rev. Lett., vol. 92, p. 101802, 2004, [arXiv:hep-ph/0308038].

[58] A. S. Belyaev, R. S. Chivukula, N. D. Christensen, H. J. He, M. Kurachi, E. H. Simmons, and M. Tanabashi, " $W_{L} W_{L}$ Scattering in Higgsless Models: Identifying Better Effective Theories," 2009, [arXiv:0907.2662 [hep-ph]].

[59] A. Birkedal, K. Matchev, and M. Perelstein, "Collider phenomenology of the Higgsless models," Phys. Rev. Lett., vol. 94, p. 191803, 2005, [arXiv:hep$\mathrm{ph} / 0412278]$.

[60] R. Malhotra, "WW fusion in Higgsless models," 2006, [arXiv:hep-ph/0611380].

[61] A. Alves, O. J. P. Eboli, D. Goncalves, M. C. Gonzalez-Garcia, and J. K. Mizukoshi, "Signals for New Spin-1 Resonances in Electroweak Gauge Boson Pair Production at the LHC," 2009, [arXiv:0907.2915 [hep-ph]]. 
[62] T. Han, H. E. Logan, B. Mukhopadhyaya, and R. Srikanth, "Neutrino masses and lepton-number violation in the littlest Higgs scenario," Phys. Rev. D, vol. 72, p. 053007, 2005, [arXiv:hep-ph/0505260].

[63] J. F. Gunion, R. Vega, and J. Wudka, "Higgs triplets in the standard model," Phys. Rev. D, vol. 42, p. 1673, 1990.

[64] K. Huitu, J. Maalampi, A. Pietila, and M. Raidal, "Doubly charged Higgs at LHC," Nucl. Phys. B, vol. 487, p. 27, 1997, [arXiv:hep-ph/9606311].

[65] J. F. Gunion, J. Grifols, A. Mendez, B. Kayser, and F. I. Olness, "Higgs Bosons In Left-Right Symmetric Models," Phys. Rev. D, vol. 40, p. 1546, 1989.

[66] G. Azuelos, K. Benslama, and J. Ferland, "Prospects for the search for a doublycharged Higgs in the left-right symmetric model with ATLAS," J. Phys. G, vol. 32, p. 73, 2006, [arXiv:hep-ph/0503096].

[67] S. Godfrey and K. Moats, "Exploring Higgs Triplet Models via Vector Boson Scattering at the LHC," Phys. Rev. D, vol. 81, p. 075026, 2010, [arXiv:1003.3033 [hep-ph]].

[68] V. D. Barger, K. Cheung, T. Han, and R. J. N. Phillips, "Strong $W^{+} W^{+}$ scattering signals at $p p$ supercolliders," Phys. Rev. D, vol. 42, p. 3052, 1990.

[69] J. Bagger et al., "The Strongly interacting $W W$ system: Gold plated modes," Phys. Rev. D, vol. 49, p. 1246, 1994, [arXiv:hep-ph/9306256].

[70] J. Bagger et al., "CERN LHC analysis of the strongly interacting $W W$ system: Gold plated modes," Phys. Rev. D, vol. 52, p. 3878, 1995, [arXiv:hep$\mathrm{ph} / 9504426]$.

[71] W. B. Kilgore, "Distinguishing among models of strong W(L) W(L) scattering at the LHC," ECONF,C960625,STC132, 1996, [arXiv:hep-ph/9610375].

[72] M.-C. Chen and S. Dawson, "One-loop Radiative Corrections to the $\rho \mathrm{Pa}$ rameter in the Littlest Higgs Model," Phys. Rev. D, vol. 70, p. 015003, 2004, [arXiv:hep-ph/0311032v3].

[73] M. S. Chanowitz and M. Golden, "Higgs boson triplets with $M_{W}=M_{Z} \cos \theta_{W}$," Phys. Lett. B, vol. 165, pp. $105-108,1985$.

[74] H. Haber and H. Logan, "Radiative Corrections to the $Z b \bar{b}$ Vertex and Constraints on Extended Higgs Sectors," Phys. Rev. D, vol. 62, p. 015011, 2000. 
[75] M. E. Peskin and T. Takeuchi, "Estimation of oblique electroweak corrections," Phys. Rev. D, vol. 46, p. 381, 1992.

[76] N. G. Deshpande, J. F. Gunion, B. Kayser, and F. I. Olness, "Left-right symmetric electroweak models with triplet Higgs," Phys. Rev. D, vol. 44, p. 837, 1991.

[77] C. Amsler et al. (Particle Data Group), "Review of Particle Physics," Phys. Lett. B, vol. 667, p. 1, 2008.

[78] J. Alwall et al., "MadGraph/MadEvent v4: The New Web Generation," JHEP, vol. 0709, p. 028, 2007, [arXiv:0706.2334 [hep-ph]].

[79] S. Dawson, "The Effective $W$ Approximation," Nucl. Phys. B, vol. 249, p. 42, 1985.

[80] J. Cornwall, D. Levin, and G. Tiktopoulos, "Derivation of gauge invariance from high-energy unitarity bounds on the S matrix," Phys. Rev. D, vol. 10, p. $1145,1974$.

[81] K. Moats, " $W Z$ Scattering at the LHC in the Littlest Higgs Model (M.Sc. thesis)," Carleton University, 2007.

[82] T. Han, D. Krohn, L. T. Wang, and W. Zhu, "New Physics Signals in Longitudinal Gauge Boson Scattering at the LHC," JHEP, vol. 1003, p. 082, 2010, [arXiv:0911.3656 [hep-ph]].

[83] V. D. Barger, A. D. Martin, and R. J. N. Phillips, "Evidence For the $t$ Quark In $p \bar{p}$ Collider Data," Phys. Lett. B, vol. 125, p. 339, 1983.

[84] V. D. Barger, T. Han, and J. Ohnemus, "Heavy Leptons At Hadron Supercolliders," Phys. Rev. D, vol. 37, p. 1174, 1988.

[85] J. Pumplin, D. R. Stump, J. Huston, H. L. Lai, P. Nadolsky, and W. K. Tung, "New generation of parton distributions with uncertainties from global QCD analysis," JHEP, vol. 0207, p. 012, 2002, [arXiv:hep-ph/0201195].

[86] P. Meade and M. Reece, "BRIDGE: Branching Ratio Inquiry/ Decay Generated Events," 2007, [arXiv:hep-ph/0703031].

[87] The ATLAS Collaboration, "Expected electron performance in the ATLAS experiment," ATL-PHYS-PUB-2011-006, 2011. 
[88] N. Arkani-Hamed, A. G. Cohen, T. Gregoire, and J. G. Wacker, "Phenomenology of electroweak symmetry breaking from theory space," JHEP, vol. 0208, p. 020, 2002, [arXiv:hep-ph/0202089].

[89] N. Arkani-Hamed, A. G. Cohen, E. Katz, A. E. Nelson, T. Gregoire, and J. G. Wacker, "The Minimal Moose for a Little Higgs," JHEP, vol. 0208, p. 021, 2002, [arXiv:hep-ph/0206020].

[90] S. Chang, "A 'littlest Higgs' model with custodial SU(2) symmetry," JHEP, vol. 0312, p. 057, (2003), [arXiv:hep-ph/0306034].

[91] C. Csaki, J. Hubisz, G. D. Kribs, P. Meade, and J. Terning, "Big corrections from a little Higgs," Phys. Rev. D, vol. 67, p. 115002, 2003, [arXiv:hep$\mathrm{ph} / 0211124]$.

[92] C. Csaki, J. Hubisz, G. D. Kribs, P. Meade, and J. Terning, "Variations of little Higgs models and their electroweak constraints," Phys. Rev. D, vol. 68, p. 035009, 2003, [arXiv:hep-ph/0303236].

[93] J. A. Casas, J. R. Espinosa, and I. Hidalgo, "Implications for new physics from fine-tuning arguments. ii: Little higgs models," JHEP, vol. 0503, p. 038, 2005, [arXiv:hep-ph/0502066].

[94] Wolfram Research, Inc., Mathematica Edition: Version 7.0. Champaign, Illinois: Wolfram Research, Inc., 2008.

[95] S. Martin, "A Supersymmetry Primer," 2011, [arXiv:hep-ph/9709356v6].

[96] J. Alwall, M. Herquet, F. Maltoni, O. Mattelaer, and T. Stelzer, "MadGraph 5: Going Beyond," JHEP, vol. 1106, p. 128, 2011, [arXiv:1106.0522 [hep-ph]].

[97] S. Godfrey, T. Grégoire, P. Kalyniak, T. Martin, and K. Moats, "Exploring the heavy quark sector of the Bestest Little Higgs model at the LHC," JHEP, vol. 1204, p. 032, 2012, [arXiv:1201.1951v3 [hep-ph]].

[98] M. E. Peskin and T. Takeuchi, "A New constraint on a strongly interacting Higgs sector," Phys. Rev. Lett., vol. 65, p. 964, 1990.

[99] G. Cacciapaglia, A. Deandrea, L. Panizzi, N. Gaur, D. Harada, and Y. Okada, "Heavy Vector-like Top Partners at the LHC and flavour constraints," 2010, [arXiv:1108.6329 [hep-ph]]. 
[100] A. J. Buras, A. Poschenrieder, and S. Uhlig, "Particle-antiparticle mixing, epsilon(K) and the unitarity triangle in the littlest Higgs model," Nucl. Phys. B, vol. 716, p. 173, 2005, [arXiv:hep-ph/0410309].

[101] M. Blanke and A. J. Buras, "A Guide to Flavour Changing Neutral Currents in the Littlest Higgs Model with T-Parity," Acta Phys. Polon. B, vol. 38, p. 2923, 2007, [arXiv:hep-ph/0703117].

[102] A. Atre, G. Azuelos, M. Carena, T. Han, E. Ozcan, J. Santiago, and G. Unel, "Model-Independent Searches for New Quarks at the LHC," JHEP, vol. 1108, p. 080, 2011, [arXiv:1102.1987 [hep-ph]].

[103] The CMS Collaboration, "Search for a Heavy Top-like Quark in the Dilepton Final State in $p p$ Collisions at $\sqrt{s}=7 \mathrm{TeV}, "$ CMS PAS EXO-11-050, 2011.

[104] S. Chatrchyan et al. [CMS Collaboration], "Search for a Vector-like Quark with Charge $2 / 3$ in $t+Z$ Events from $p p$ Collisions at $\sqrt{s}=7 \mathrm{TeV}$," 2011, [arXiv:1109.4985 [hep-ex]].

[105] The CMS Collaboration, "Search for a Top-like Quark Decaying to a Top Quark and a $Z$ boson in $p p$ Collisions at $\sqrt{s}=7 \mathrm{TeV}, "$ CMS PAS EXO-11-005, 2011.

[106] G. Aad et al. [ATLAS Collaboration], "Search for Pair Production of a Heavy Up-Type Quark Decaying to a $W$ Boson and a $b$ Quark in the lepton + jets Channel with the ATLAS Detector," Phys. Rev. Lett., vol. 108, p. 261802, 2012, [arXiv:1202.3076v2 [hep-ex]].

[107] E. L. Berger and Q. H. Cao, "Next-to-Leading Order Cross Sections for New Heavy Fermion Production at Hadron Colliders," Phys. Rev. D, vol. 81, p. 035006, 2010, [arXiv:0909.3555 [hep-ph]].

[108] D. Stump, J. Huston, J. Pumplin, W.-K. Tung, H.-L. Lai, S. Kuhlmann, and J. Owens, "Inclusive jet production, parton distributions, and the search for new physics," JHEP, vol. 0310, p. 046, 2003, [arXiv:hep-ph/0303013].

[109] J. M. Campbell, R. Frederix, F. Maltoni, and F. Tramontano, "NLO predictions for t-channel production of single top and fourth generation quarks at hadron colliders," JHEP, vol. 1088, p. 042, 2009, [arXiv:0907.3933 [hep-ph]].

[110] The ATLAS Collaboration, "Measurement of the t-channel Single Top-Quark Production Cross Section in $0.70 \mathrm{fb}^{-1}$ of $p p$ Collisions at $\sqrt{s}=7 \mathrm{TeV}$ collected with the ATLAS detector," ATLAS-CONF-2011-101, 2011. 
[111] R. Contino and G. Servant, "Discovering the top partners at the LHC using same-sign dilepton final states," JHEP, vol. 0806, p. 026, 2008, [arXiv:0801.1679v2 [hep-ph]].

[112] J. Mrazek and A. Wulzer, "A Strong Sector at the LHC: Top Partners in SameSign Dileptons," Phys. Rev. D, vol. 81, p. 075006, 2010, [arXiv:0909.3977v2 [hep-ph]].

[113] G. Aad et al. [ATLAS Collaboration], "Expected performance of the ATLAS experiment: detector, trigger and physics," CERN-OPEN-2008-020, 2009, [arXiv:0901.0512v4 [hep-ex]].

[114] G. Aad et al. [ATLAS Collaboration], "Electron performance measurements with the ATLAS detector using the 2010 LHC proton-proton collision data," Eur. Phys. J. C, pp. 1-46, 2012, [arXiv:1110.3174v2 [hep-ex]].

[115] T. Sjöstrand, S. Mrenna, and P. Skands, "PYTHIA 6.4 physics and manual," JHEP, vol. 05, p. 026, 2006, [arXiv:hep-ph/0603175v2].

[116] D. E. Kaplan, K. Rehermann, M. D. Schwartz, and B. Tweedie, "Top Tagging: A Method for Identifying Boosted Hadronically Decaying Top Quarks," Phys. Rev. Lett., vol. 101, p. 142001, 2008, [arXiv:0806.0848v2 [hep-ph]].

[117] J. Sakurai, Modern Quantum Mechanics. Addison-Wesley, 1994. 